\title{
A stereo- and regioselective 1,3-dipolar cycloaddition of the stable ninhydrin-derived azomethine ylide to cyclopropenes. Trapping of unstable cyclopropene dipolarophiles
}

\author{
Alexander S. Filatov, ${ }^{\text {a }}$ Siqi Wang, ${ }^{\text {a }}$ Olesya V. Khoroshilova, ${ }^{a}$ Stanislav V. Lozovskiy, ${ }^{\text {a }}$ \\ Anna G. Larina, ${ }^{a}$ Vitali M. Boitsov, ${ }^{\text {b,c }}$ Alexander V. Stepakov*a,d \\ ${ }^{a}$ Saint Petersburg State University, Institute of Chemistry, Universitetskii pr. 26, 198504, St. Petersburg, Russian \\ Federation \\ ${ }^{\mathrm{b}}$ Saint Petersburg Academic University, ul. Khlopina 8/3, 194021, St. Petersburg, Russian Federation \\ ${ }^{c}$ Pavlov First Saint Petersburg State Medicinal University, ul. L'va Tolstogo 6/8, 197022, St. Petersburg, Russian \\ Federation \\ ${ }^{d}$ Saint Petersburg State Institute of Technology, Moskovskii pr. 26, 190013, St. Petersburg, Russian Federation \\ *Corresponding author: A. V. Stepakov (alstep@yandex.ru)
}

\section{Table of contents}

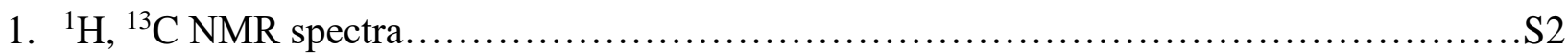

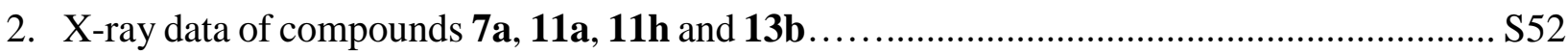

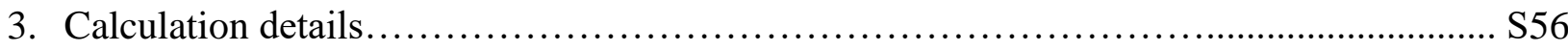

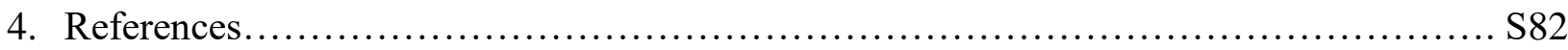




\section{1. ${ }^{1} \mathrm{H},{ }^{13} \mathrm{C}$ NMR spectra}

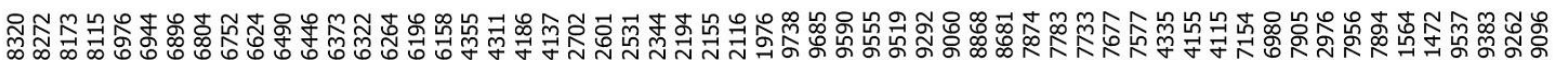

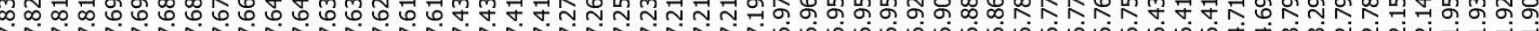
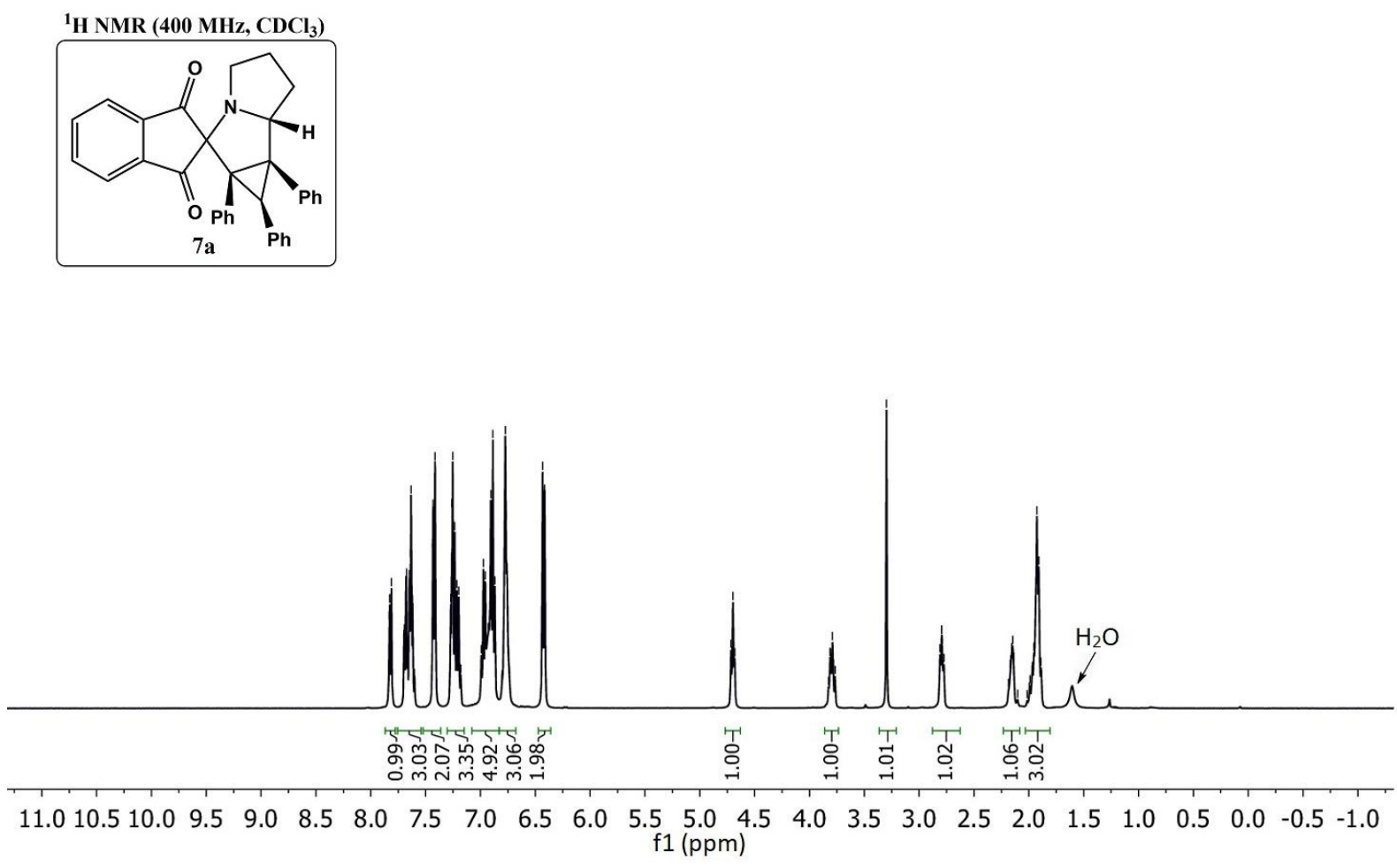

Figure S1. ${ }^{1} \mathrm{H}$ NMR spectrum of compound $7 \mathbf{a}\left(\mathrm{CDCl}_{3}, 400 \mathrm{MHz}\right)$

空品

กิ่

${ }^{13} \mathrm{C}$ NMR (101 MHz, $\left.\mathrm{CDCl}_{3}\right)$

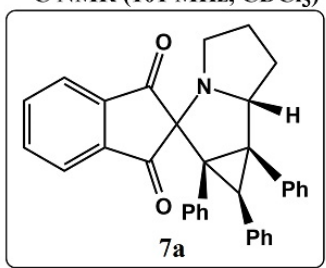

7 a

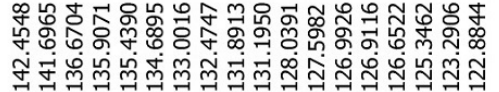
-

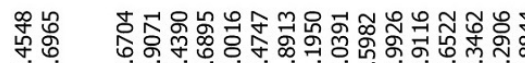
要
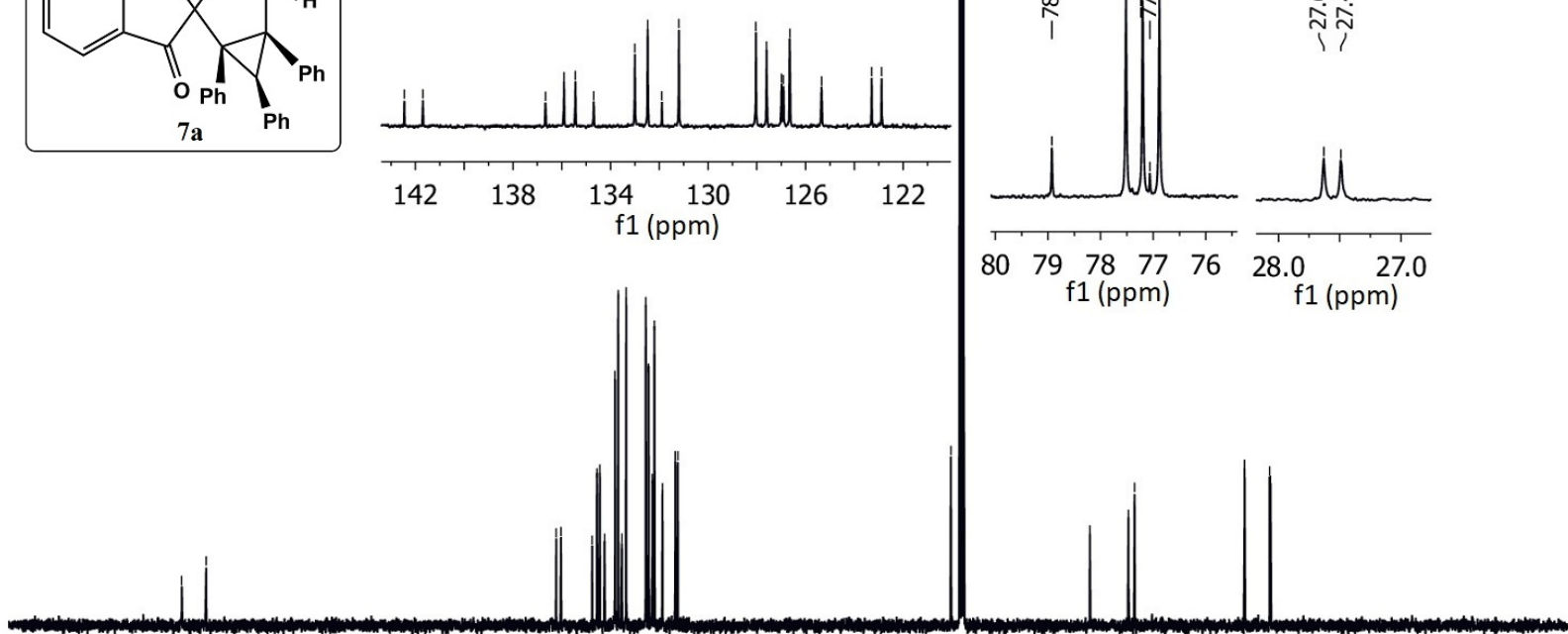

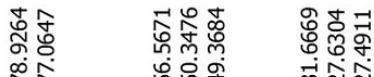

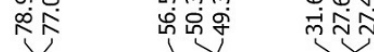

$\mathrm{CDCl}_{3}$

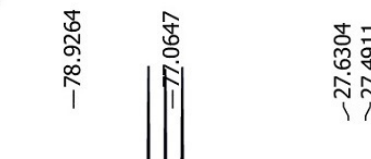

$\begin{array}{llllllllllllllllllllllll}220 & 210 & 200 & 190 & 180 & 170 & 160 & 150 & 140 & 130 & 120 & \begin{array}{l}110 \\ \mathrm{f} 1(\mathrm{ppm})\end{array}\end{array}$

Figure S2. ${ }^{13} \mathrm{C}$ NMR spectrum of compound $7 \mathbf{a}\left(\mathrm{CDCl}_{3}, 101 \mathrm{MHz}\right)$ 
${ }^{1} \mathrm{H}$ NMR (400 MHz, $\left.\mathrm{CDCl}_{3}\right)$

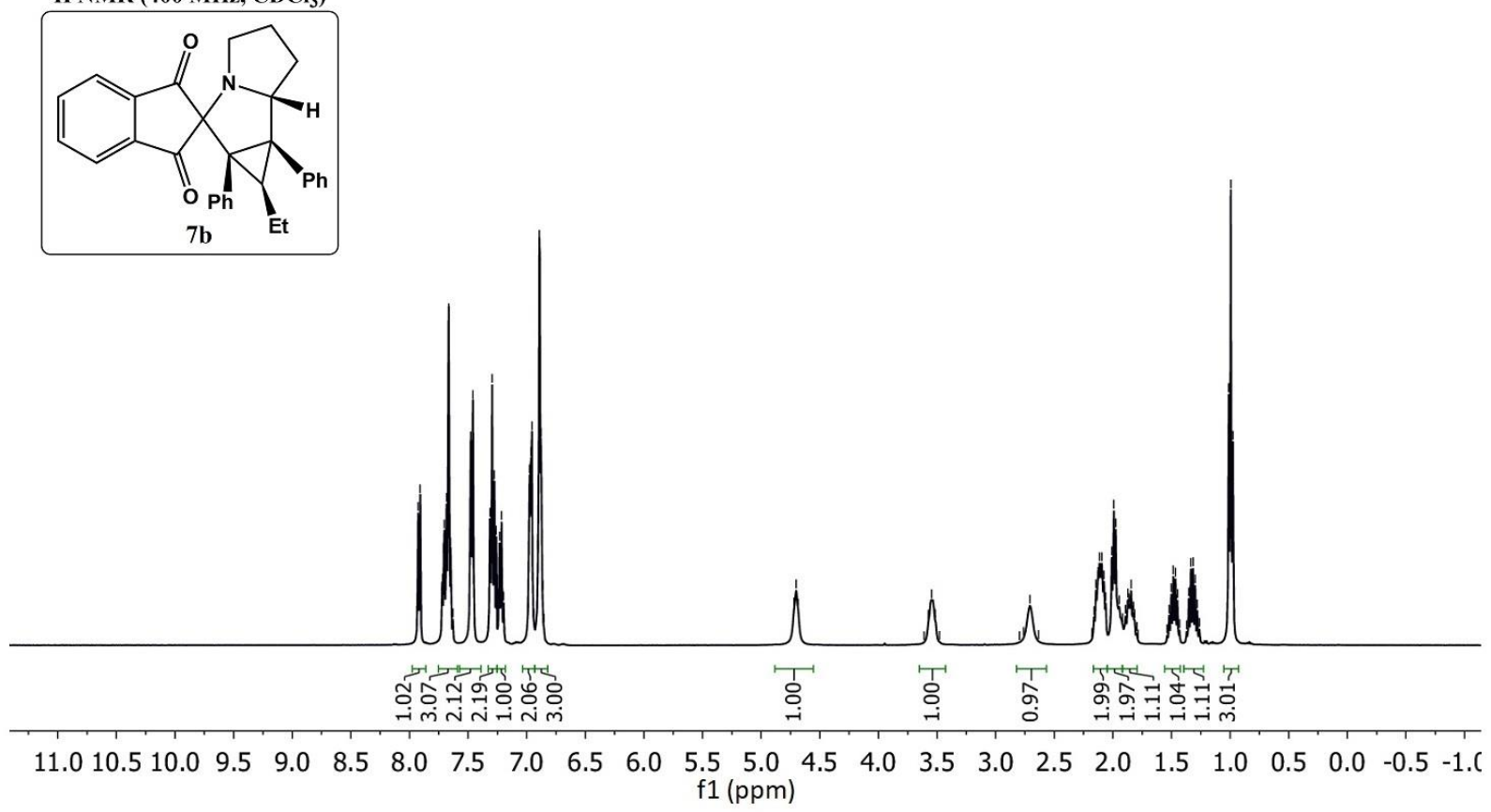

Figure S3. ${ }^{1} \mathrm{H}$ NMR spectrum of compound $7 \mathbf{b}\left(\mathrm{CDCl}_{3}, 400 \mathrm{MHz}\right)$

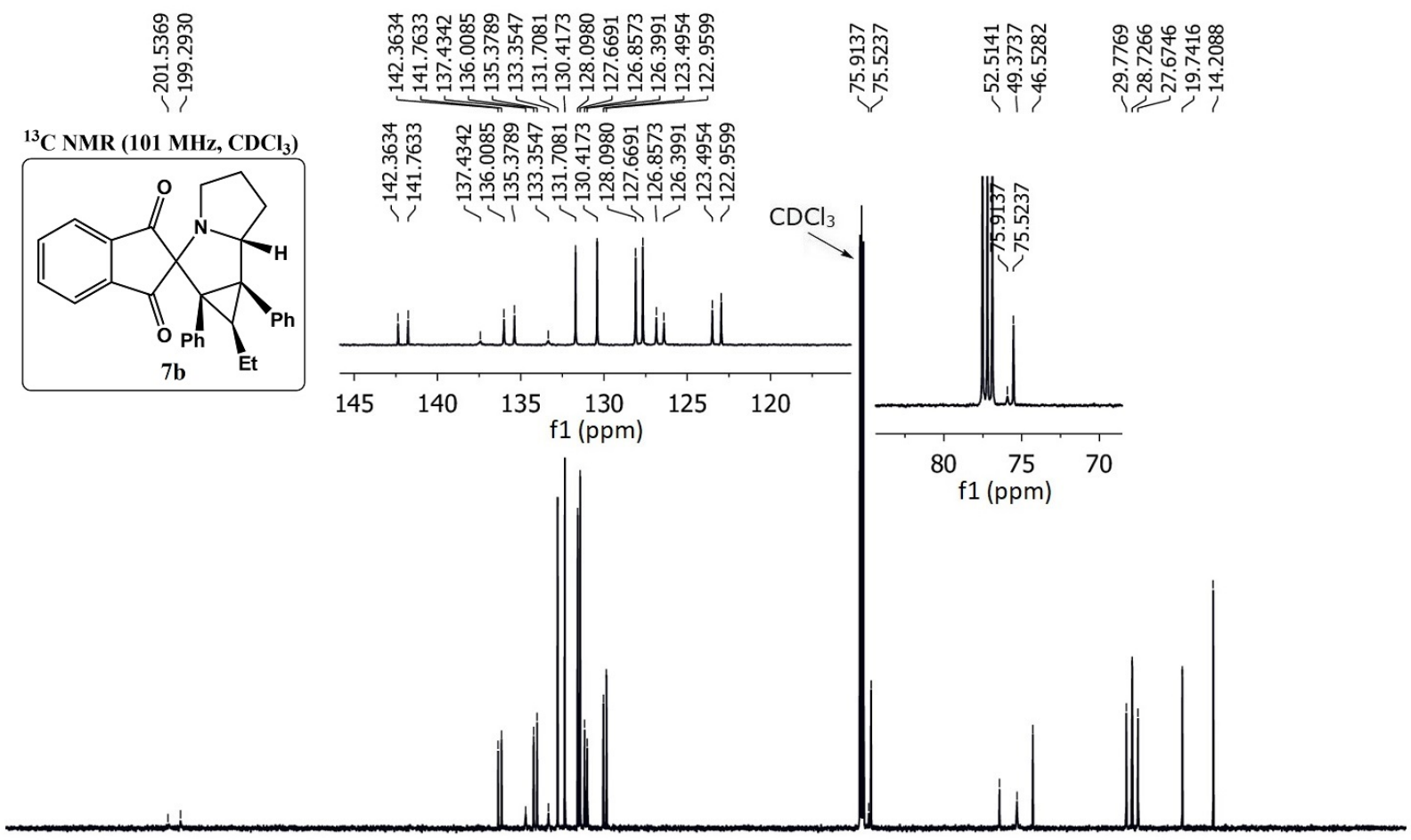

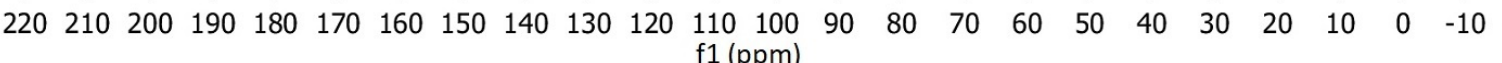

Figure S4. ${ }^{13} \mathrm{C}$ NMR spectrum of compound $\mathbf{7 b}\left(\mathrm{CDCl}_{3}, 101 \mathrm{MHz}\right)$ 


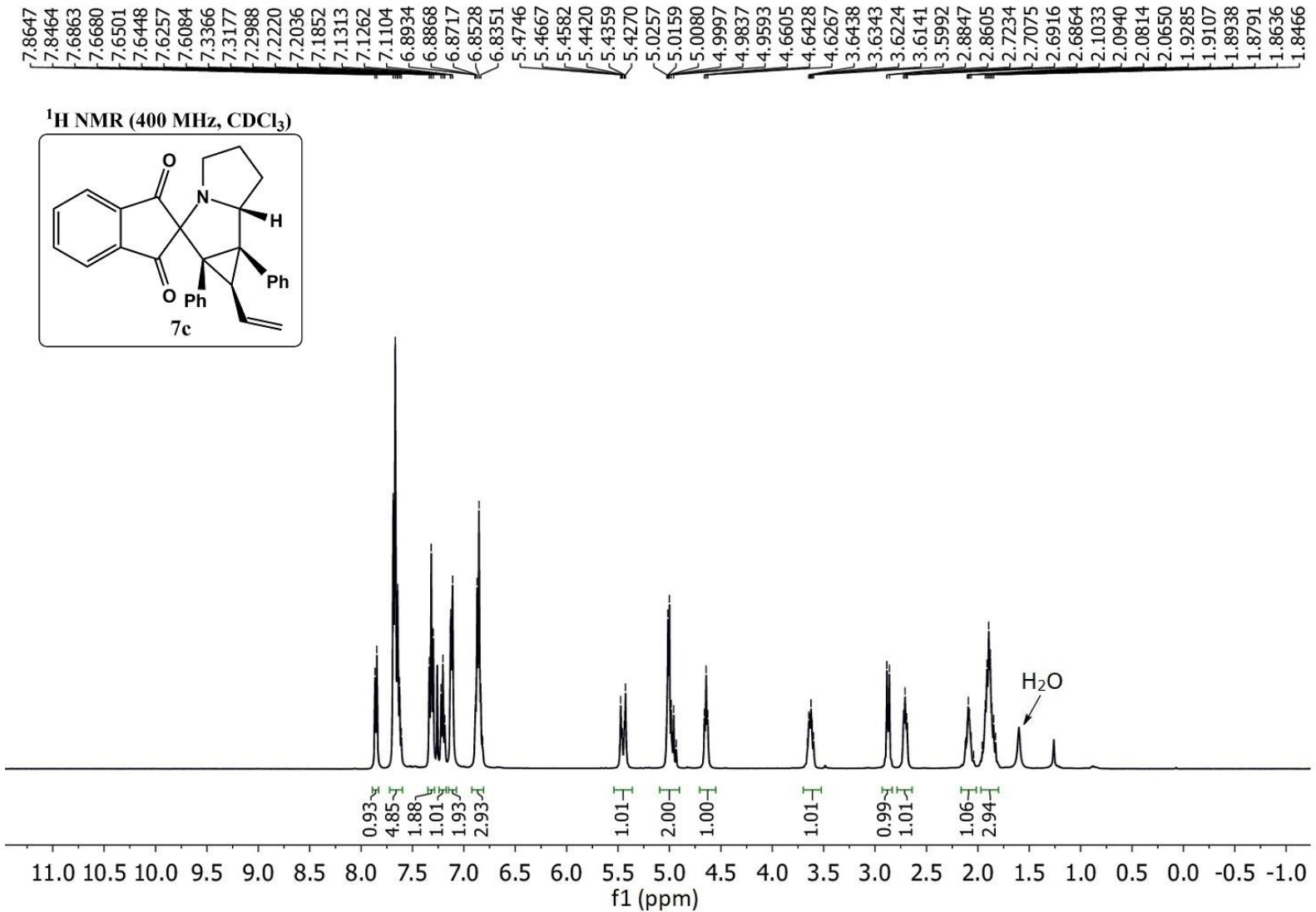

Figure S5. ${ }^{1} \mathrm{H}$ NMR spectrum of compound $7 \mathbf{c}\left(\mathrm{CDCl}_{3}, 400 \mathrm{MHz}\right)$

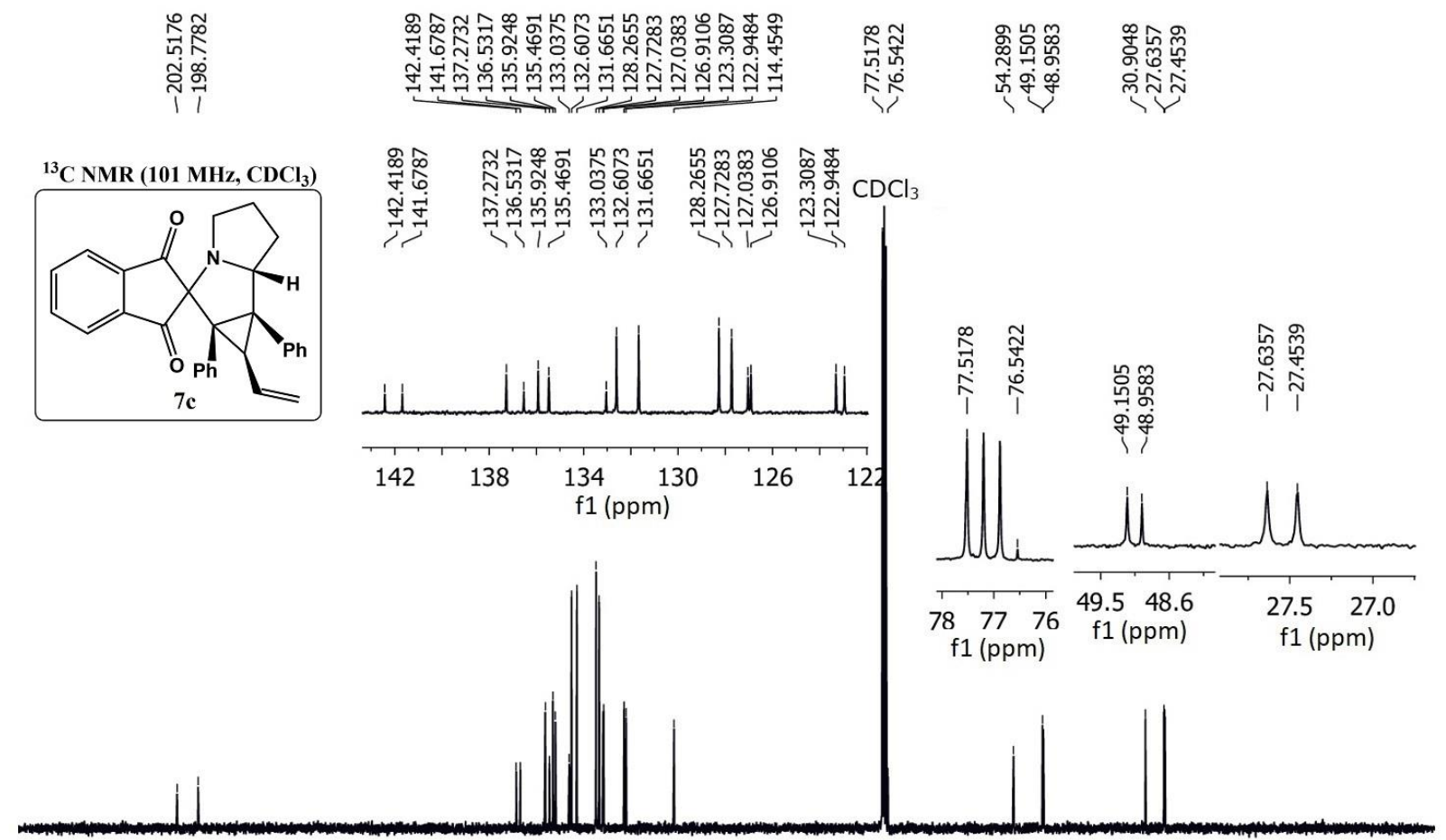

$\begin{array}{llllllllllllllllllllllll}220 & 210 & 200 & 190 & 180 & 170 & 160 & 150 & 140 & 130 & 120 & \begin{array}{l}110 \\ \mathrm{f} 1(\mathrm{ppm})\end{array} & 90 & 80 & 70 & 60 & 50 & 40 & 30 & 20 & 10 & 0 & -10\end{array}$

Figure S6. ${ }^{13} \mathrm{C} \mathrm{NMR}$ spectrum of compound $7 \mathrm{c}\left(\mathrm{CDCl}_{3}, 101 \mathrm{MHz}\right)$ 

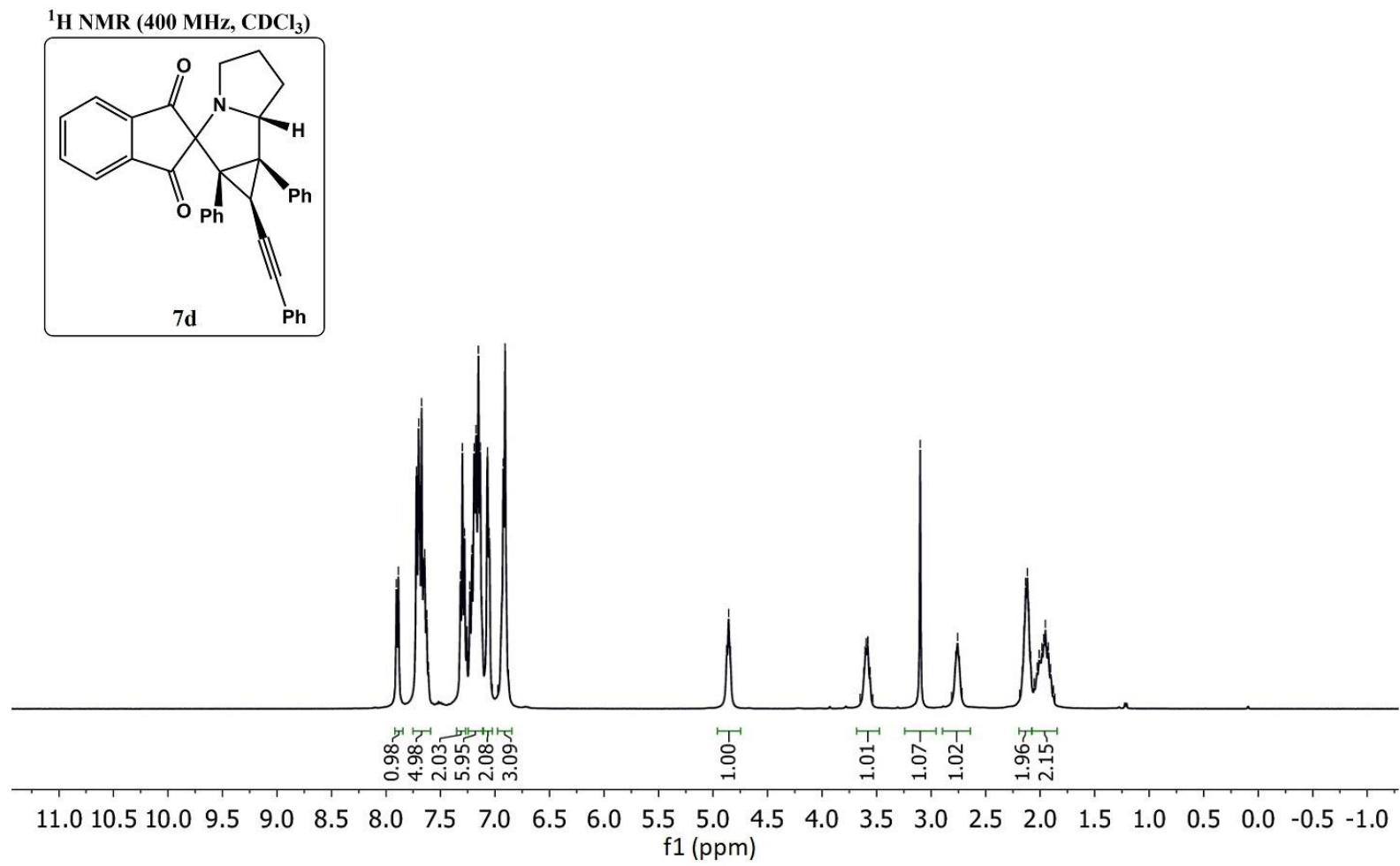

Figure S7. ${ }^{1} \mathrm{H}$ NMR spectrum of compound $7 \mathbf{d}\left(\mathrm{CDCl}_{3}, 400 \mathrm{MHz}\right)$

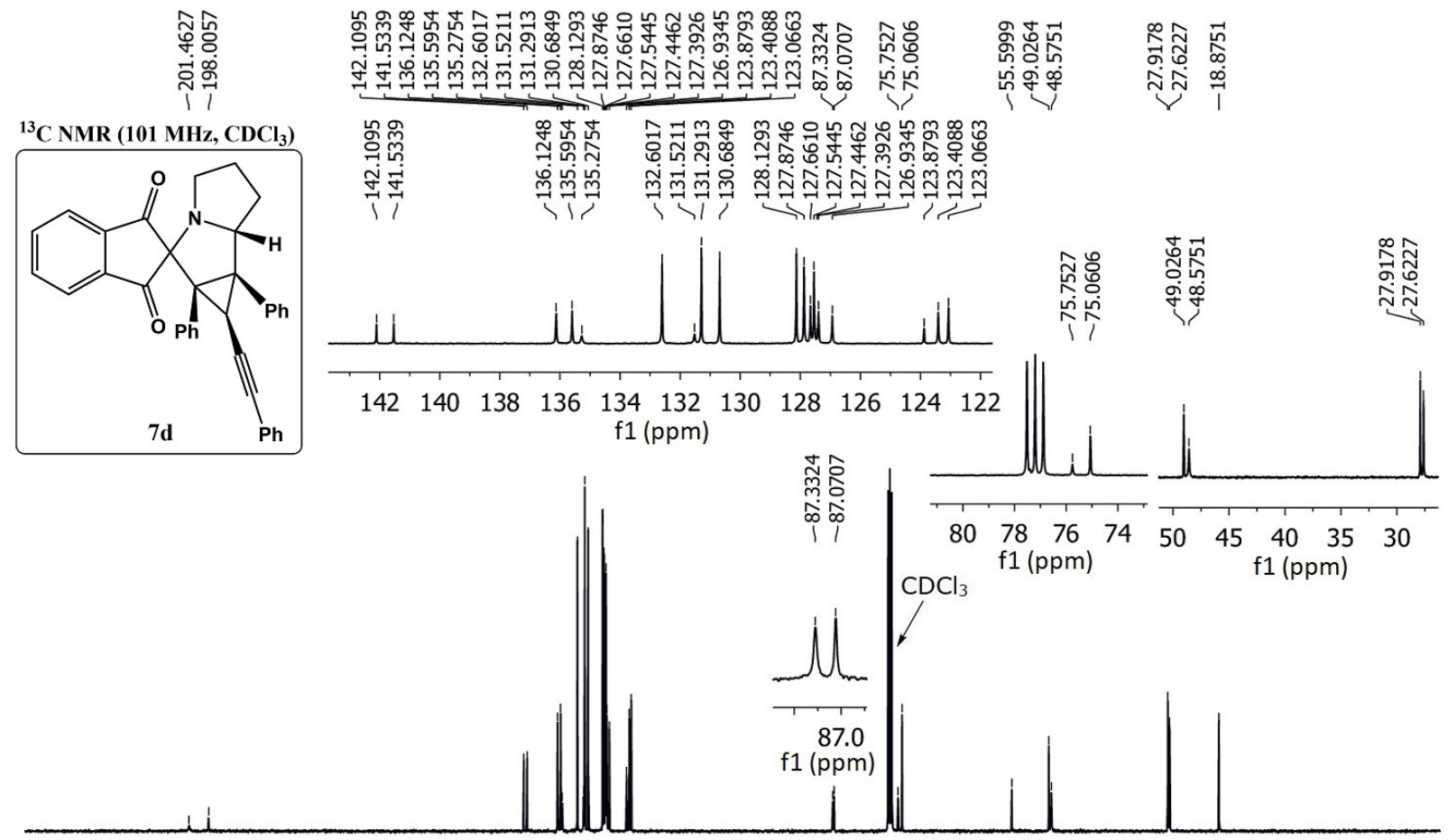

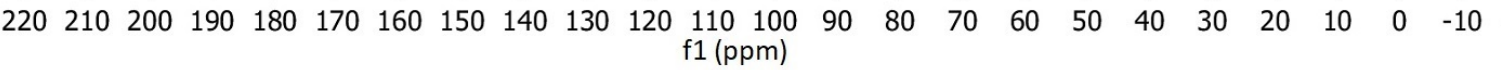

Figure S8. ${ }^{13} \mathrm{C}$ NMR spectrum of compound $7 \mathbf{d}\left(\mathrm{CDCl}_{3}, 101 \mathrm{MHz}\right)$ 

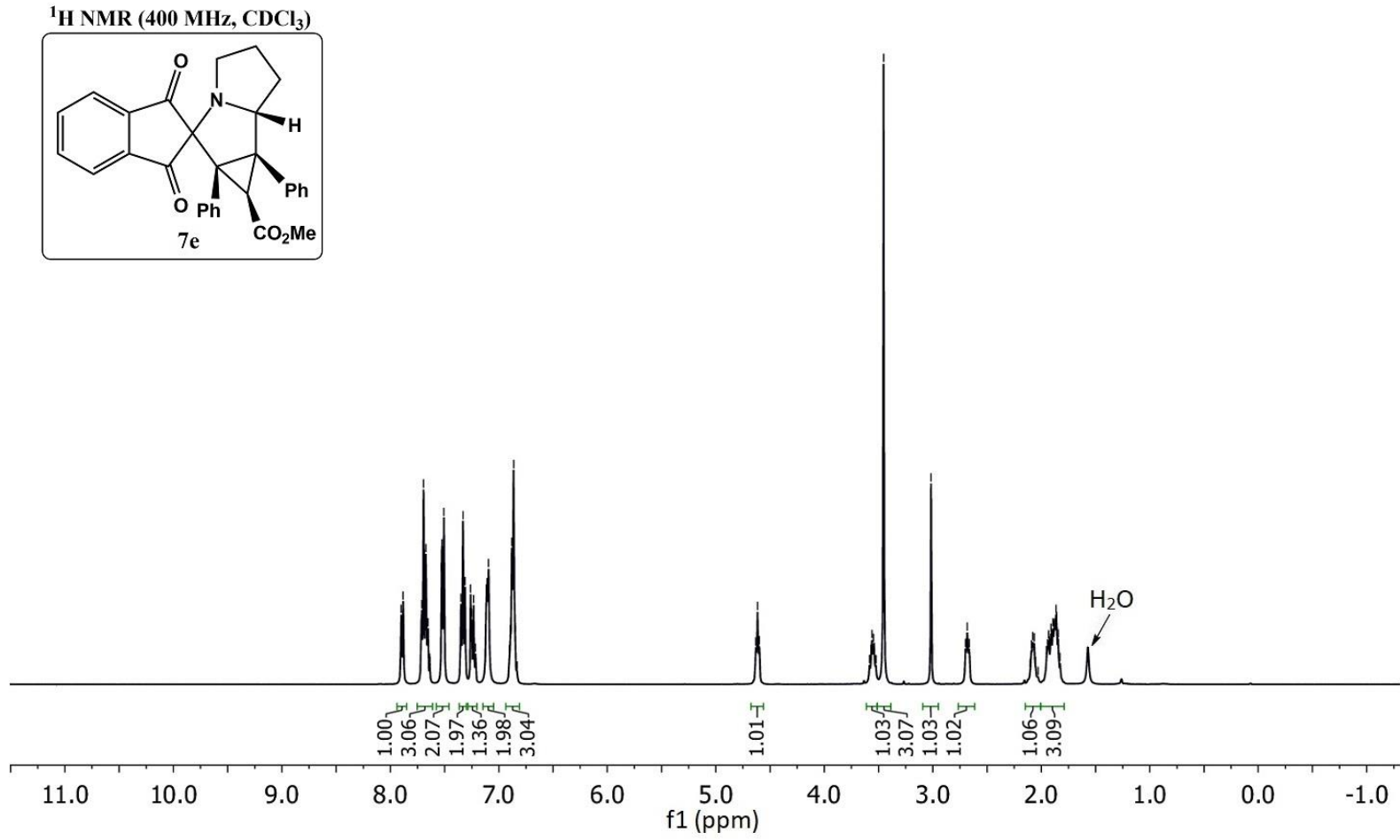

Figure S9. ${ }^{1} \mathrm{H}$ NMR spectrum of compound $7 \mathbf{e}\left(\mathrm{CDCl}_{3}, 400 \mathrm{MHz}\right)$

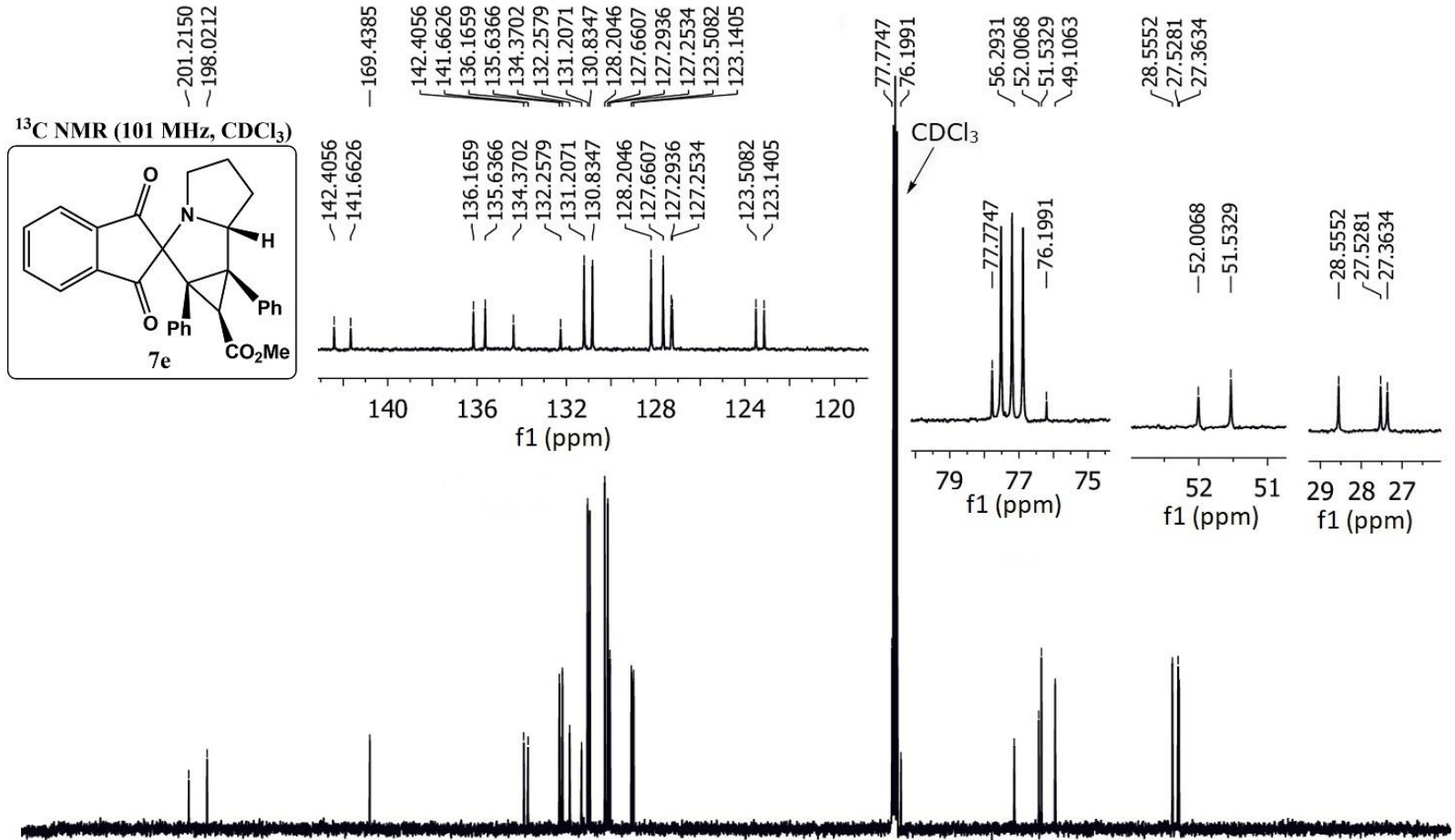

$\begin{array}{lllllllllllllllllllll}220 & 210 & 200 & 190 & 180 & 170 & 160 & 150 & 140 & 130 & 120 & \begin{array}{l}110 \\ \mathrm{f} 1(\mathrm{ppm})\end{array}\end{array}$

Figure S10. ${ }^{13} \mathrm{C}$ NMR spectrum of compound $7 \mathbf{e}\left(\mathrm{CDCl}_{3}, 101 \mathrm{MHz}\right)$ 
${ }^{1}$ H NMR (400 MHz, DMSO- $d_{6}$ )
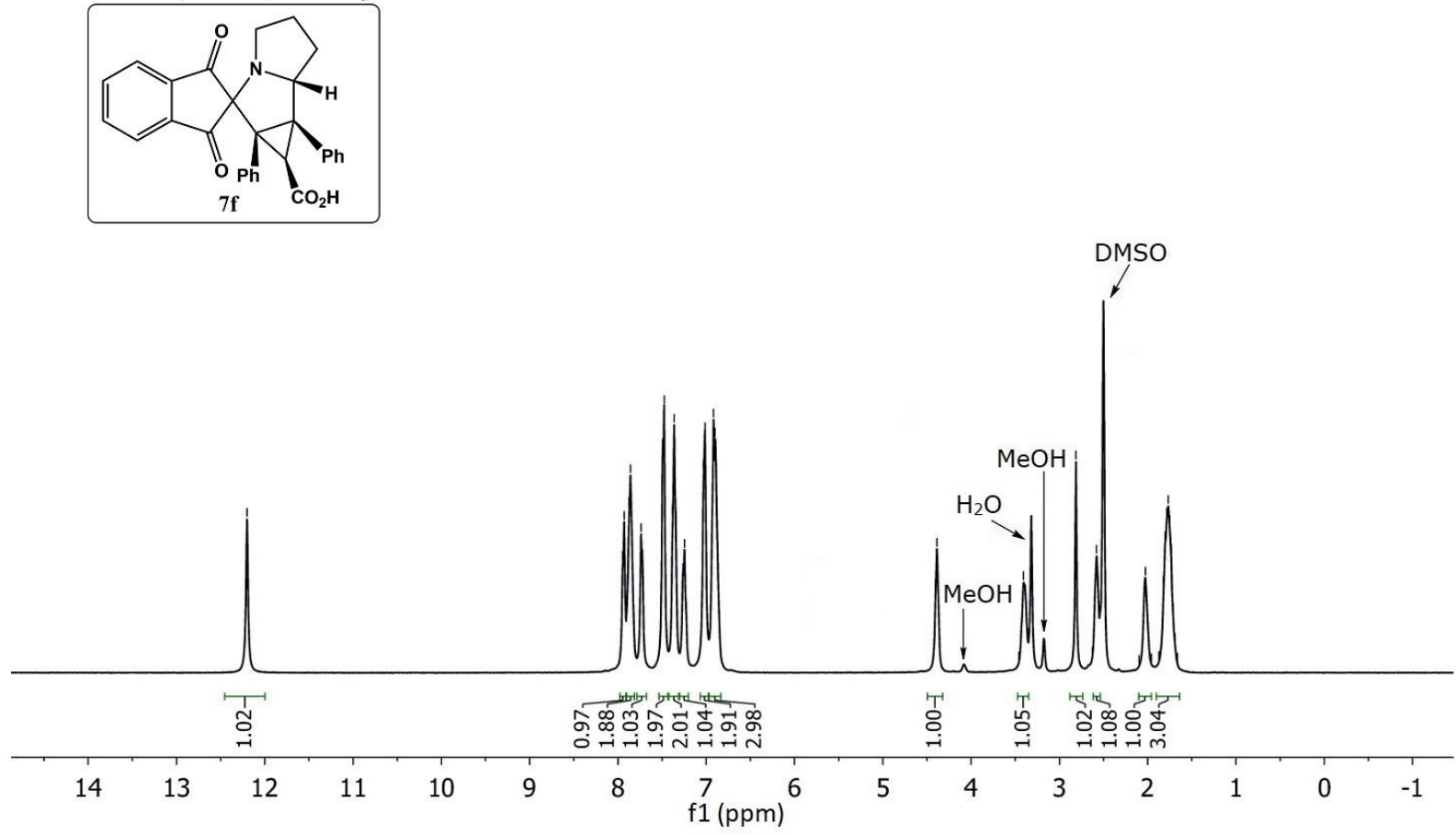

Figure S11. ${ }^{1} \mathrm{H}$ NMR spectrum of compound $7 \mathbf{f}\left(\mathrm{DMSO}-d_{6}, 400 \mathrm{MHz}\right)$

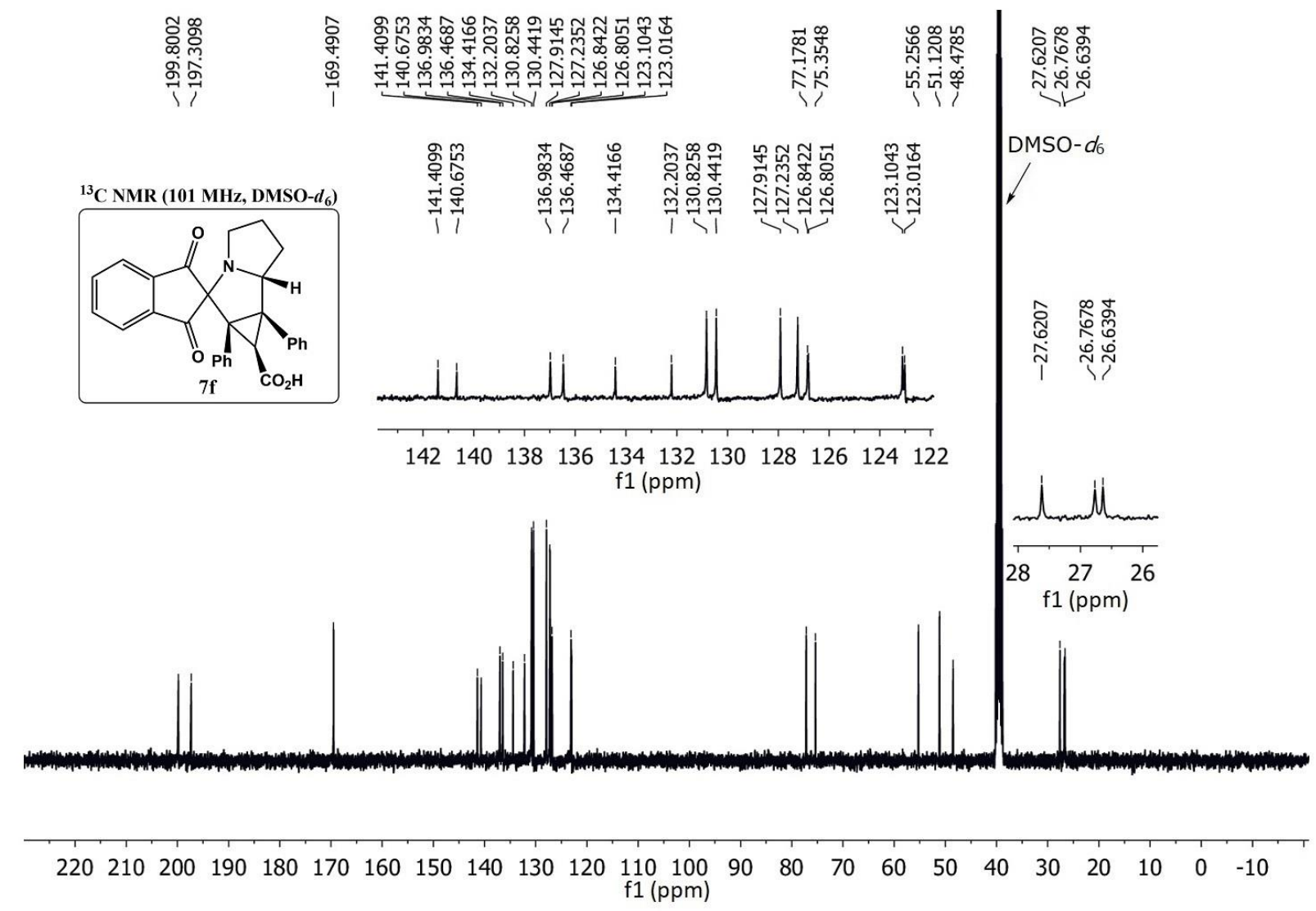

Figure S12. ${ }^{13} \mathrm{C}$ NMR spectrum of compound $7 \mathbf{f}\left(\mathrm{DMSO}-d_{6}, 101 \mathrm{MHz}\right)$ 
${ }^{1} \mathrm{H}$ NMR (400 MHz, $\left.\mathrm{CDCl}_{3}\right)$
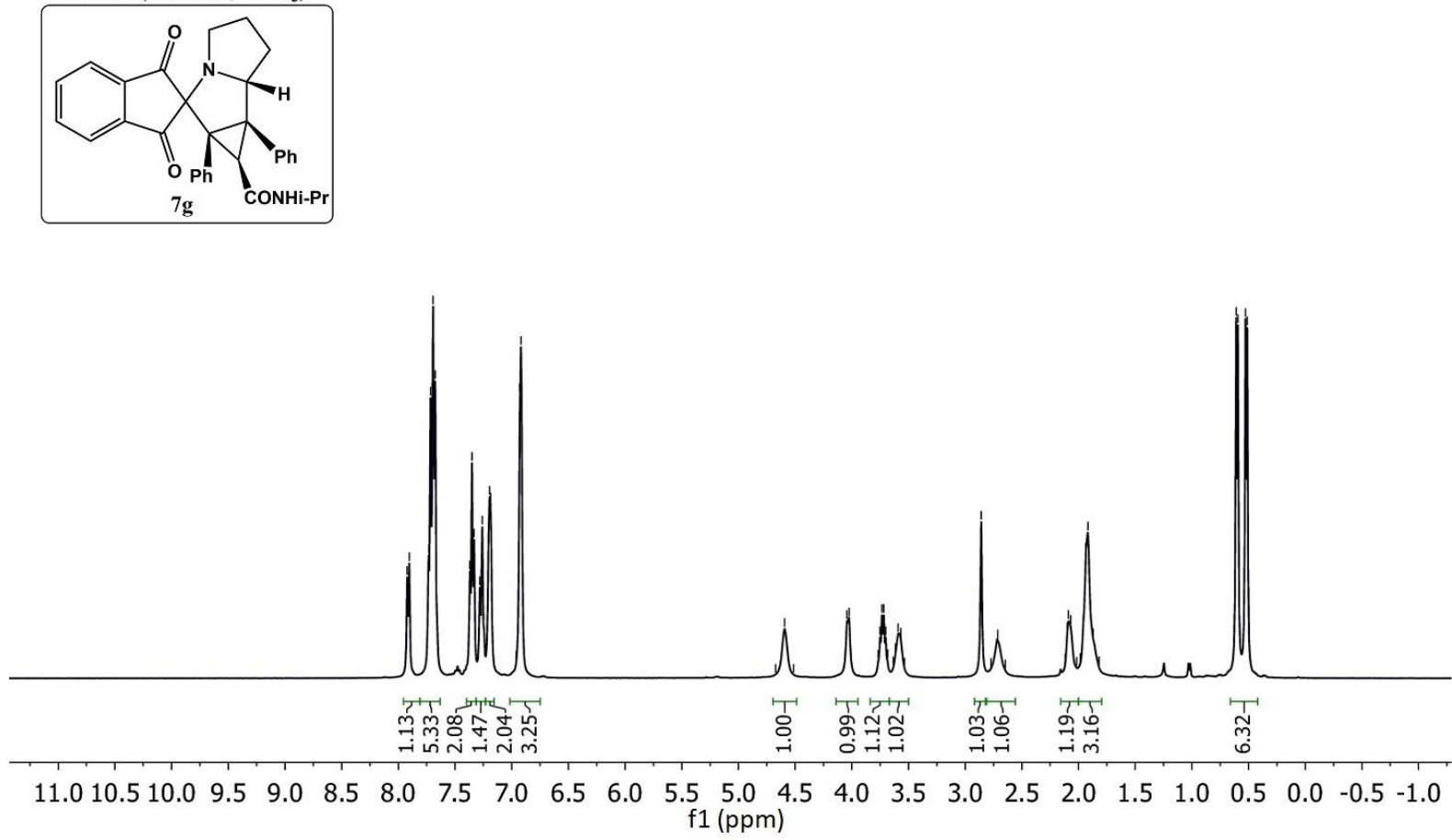

Figure S13. ${ }^{1} \mathrm{H}$ NMR spectrum of compound $7 \mathbf{g}\left(\mathrm{CDCl}_{3}, 400 \mathrm{MHz}\right)$

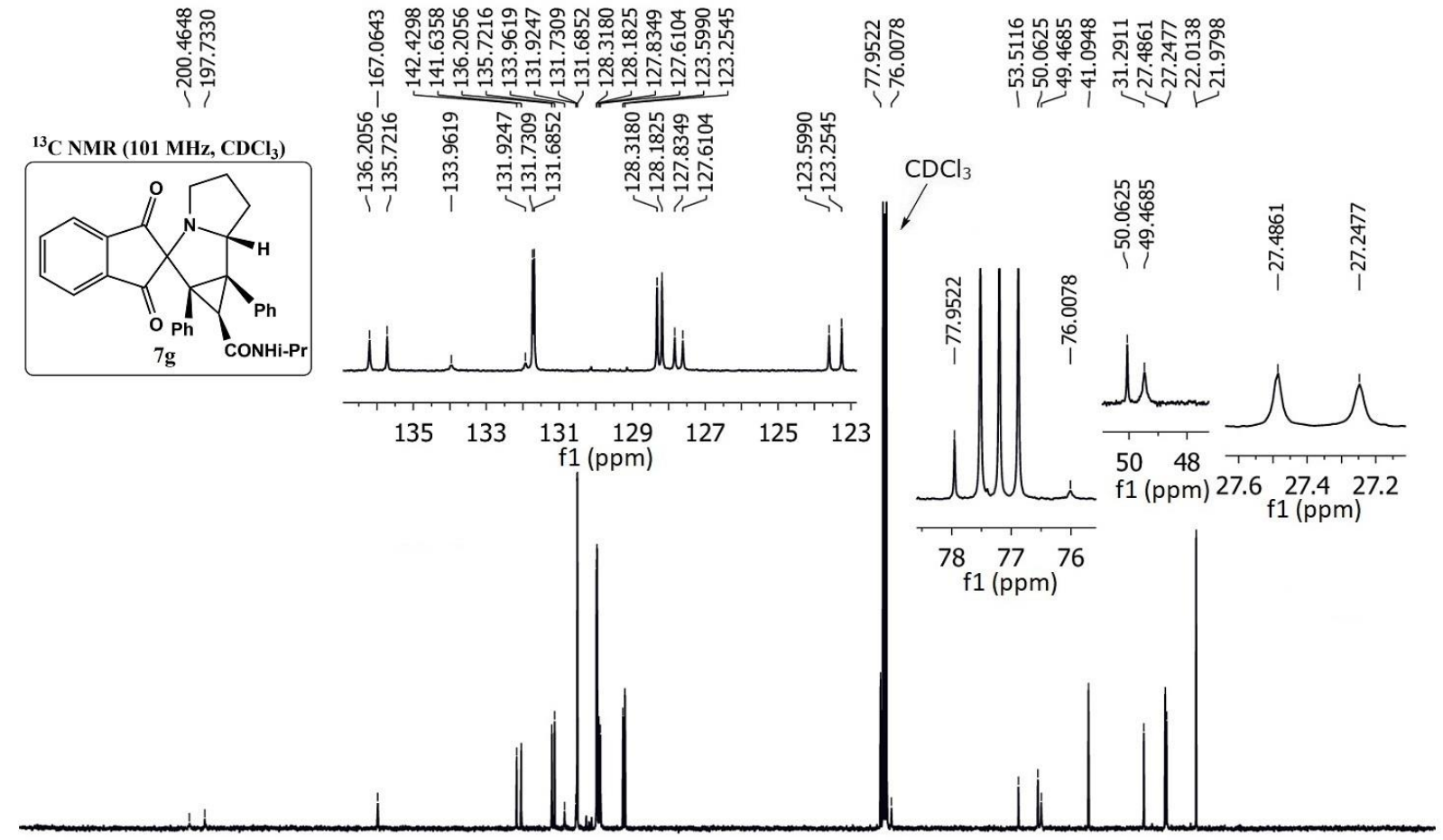

$\begin{array}{llllllllllllllllllllllll}220 & 210 & 200 & 190 & 180 & 170 & 160 & 150 & 140 & 130 & 120 & \begin{array}{l}110 \\ \mathrm{f} 1(\mathrm{ppm})\end{array} & 90 & 80 & 70 & 60 & 50 & 40 & 30 & 20 & 10 & 0 & -10\end{array}$

Figure S14. ${ }^{13} \mathrm{C}$ NMR spectrum of compound $7 \mathrm{~g}\left(\mathrm{CDCl}_{3}, 101 \mathrm{MHz}\right)$ 
${ }^{1} \mathrm{H}$ NMR (400 MHz, $\mathrm{CDCl}_{3}$ )
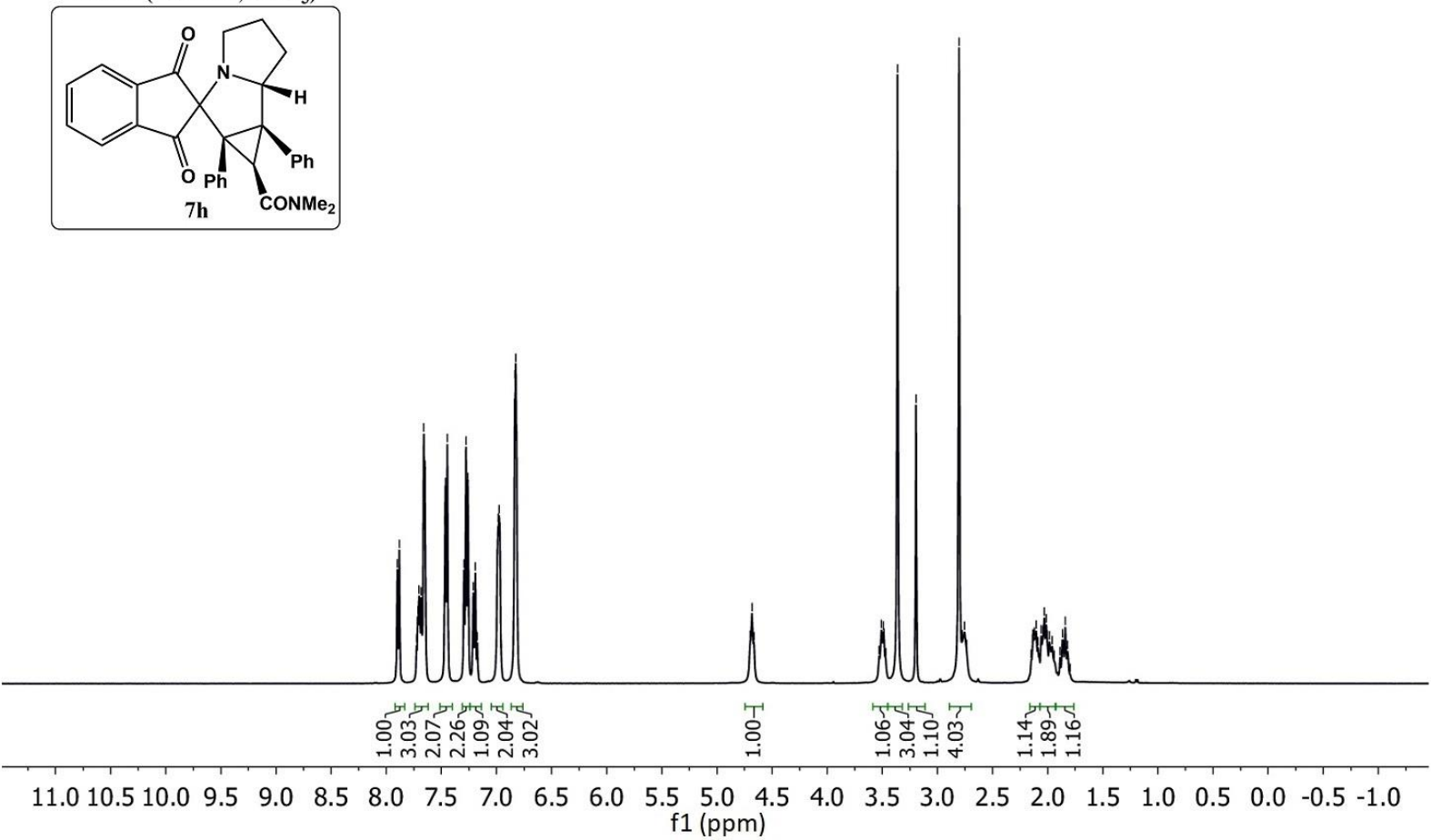

Figure S15. ${ }^{1} \mathrm{H}$ NMR spectrum of compound $7 \mathbf{h}\left(\mathrm{CDCl}_{3}, 400 \mathrm{MHz}\right)$

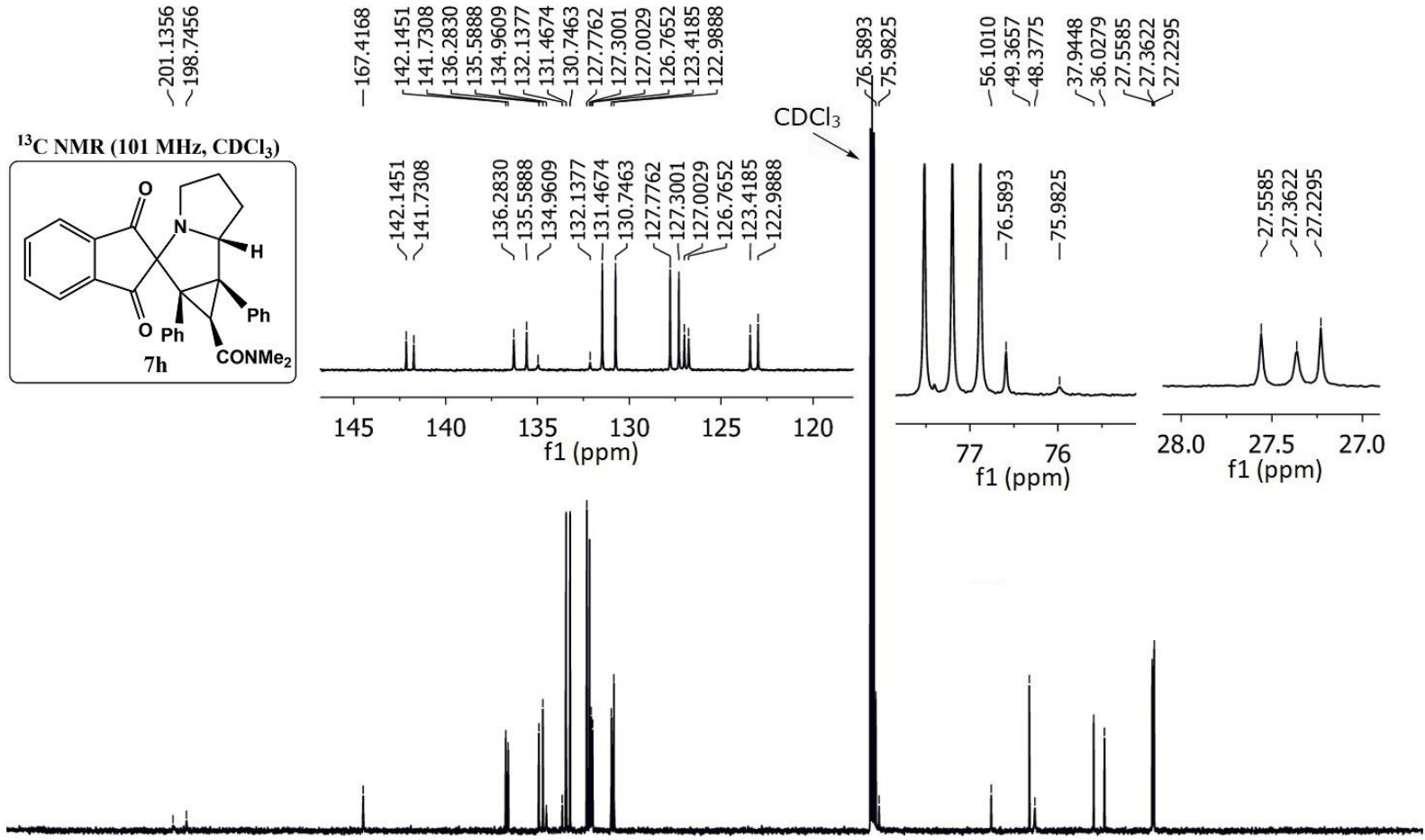

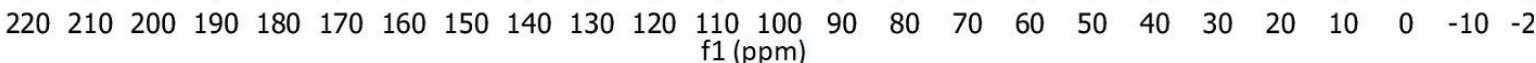

Figure S16. ${ }^{13} \mathrm{C}$ NMR spectrum of compound $7 \mathbf{h}\left(\mathrm{CDCl}_{3}, 101 \mathrm{MHz}\right)$ 

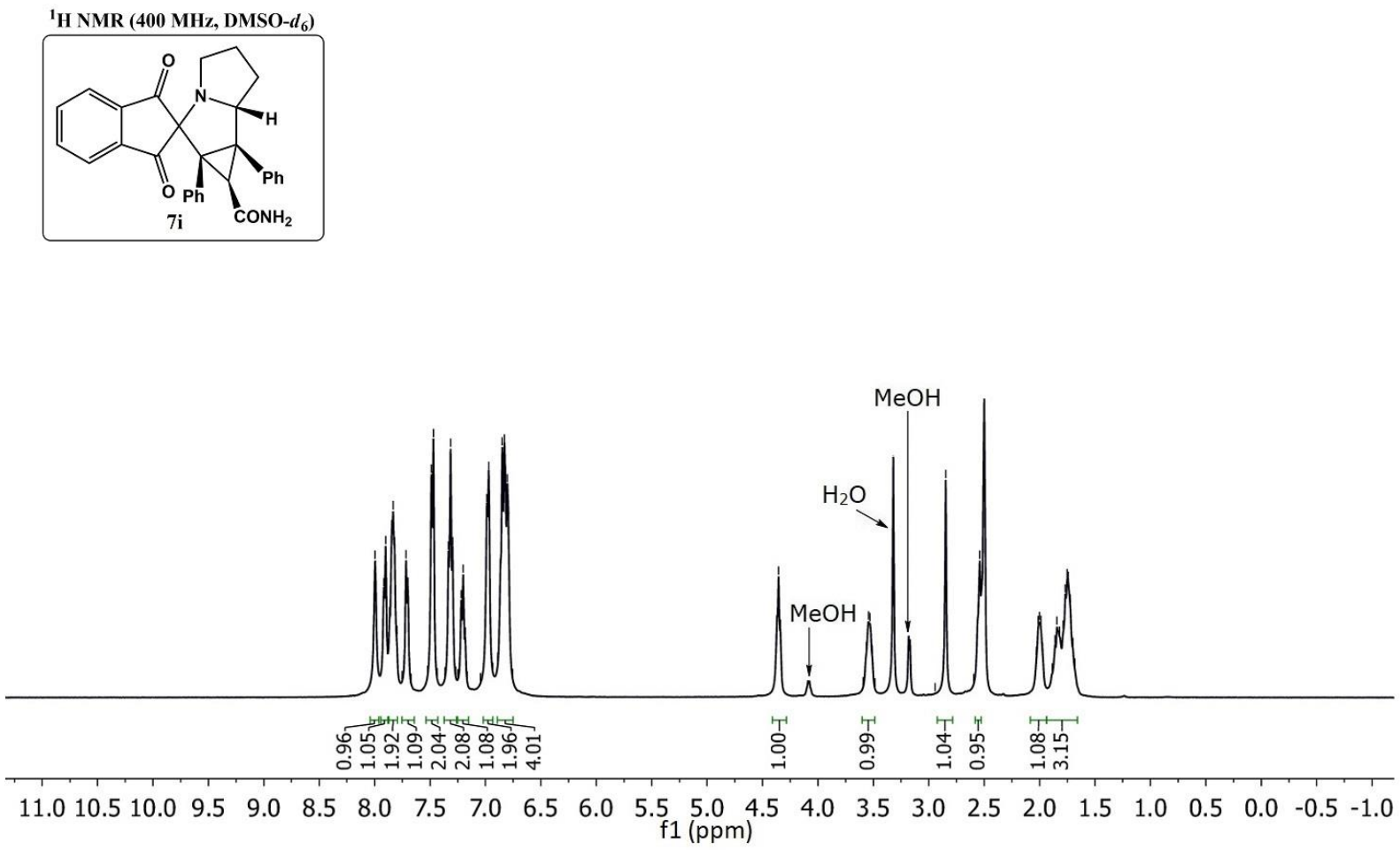

Figure S17. ${ }^{1} \mathrm{H}$ NMR spectrum of compound 7i (DMSO- $\left.d_{6}, 400 \mathrm{MHz}\right)$

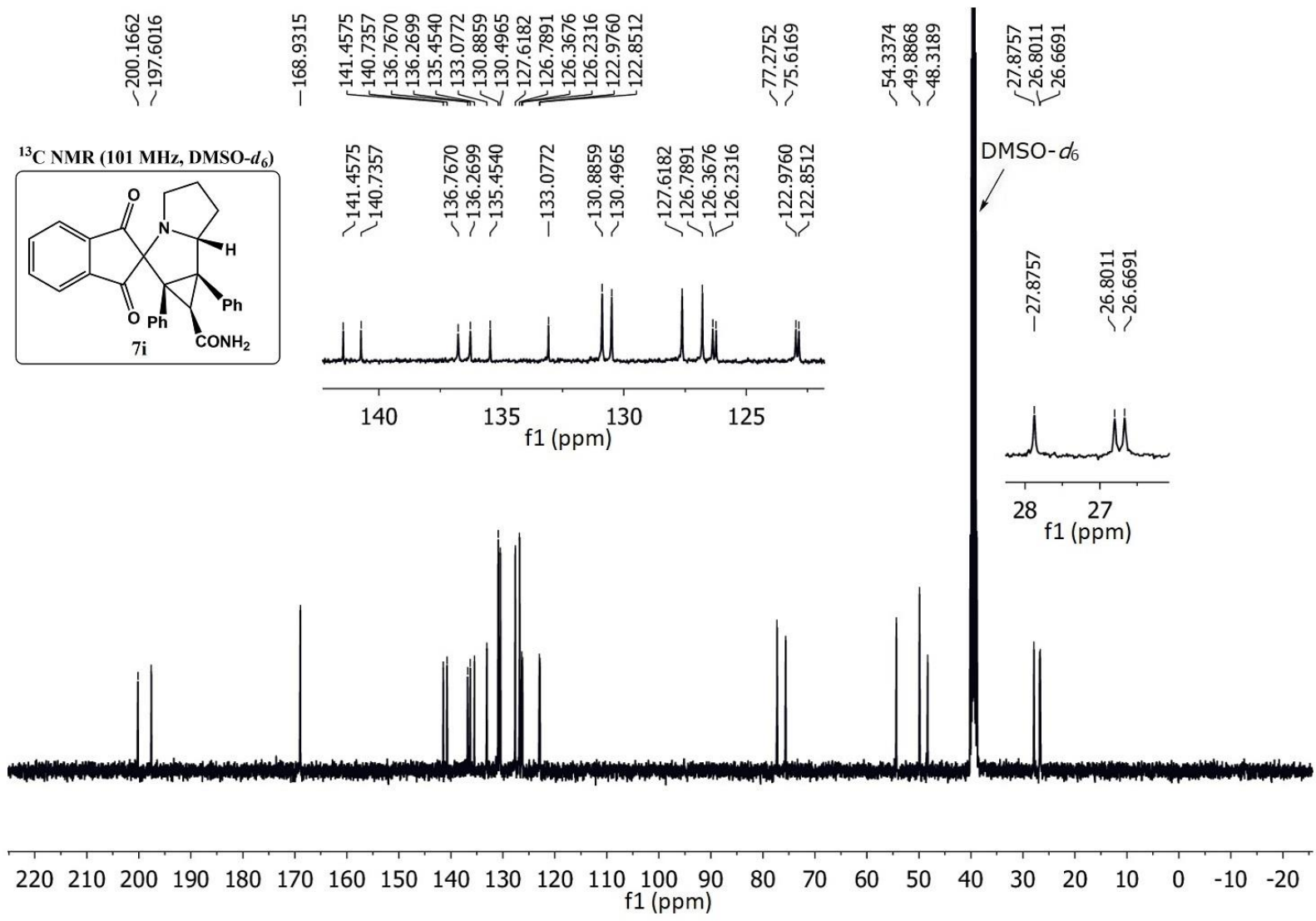

Figure S18. ${ }^{13} \mathrm{C}$ NMR spectrum of compound 7i (DMSO- $\left.d_{6}, 101 \mathrm{MHz}\right)$ 

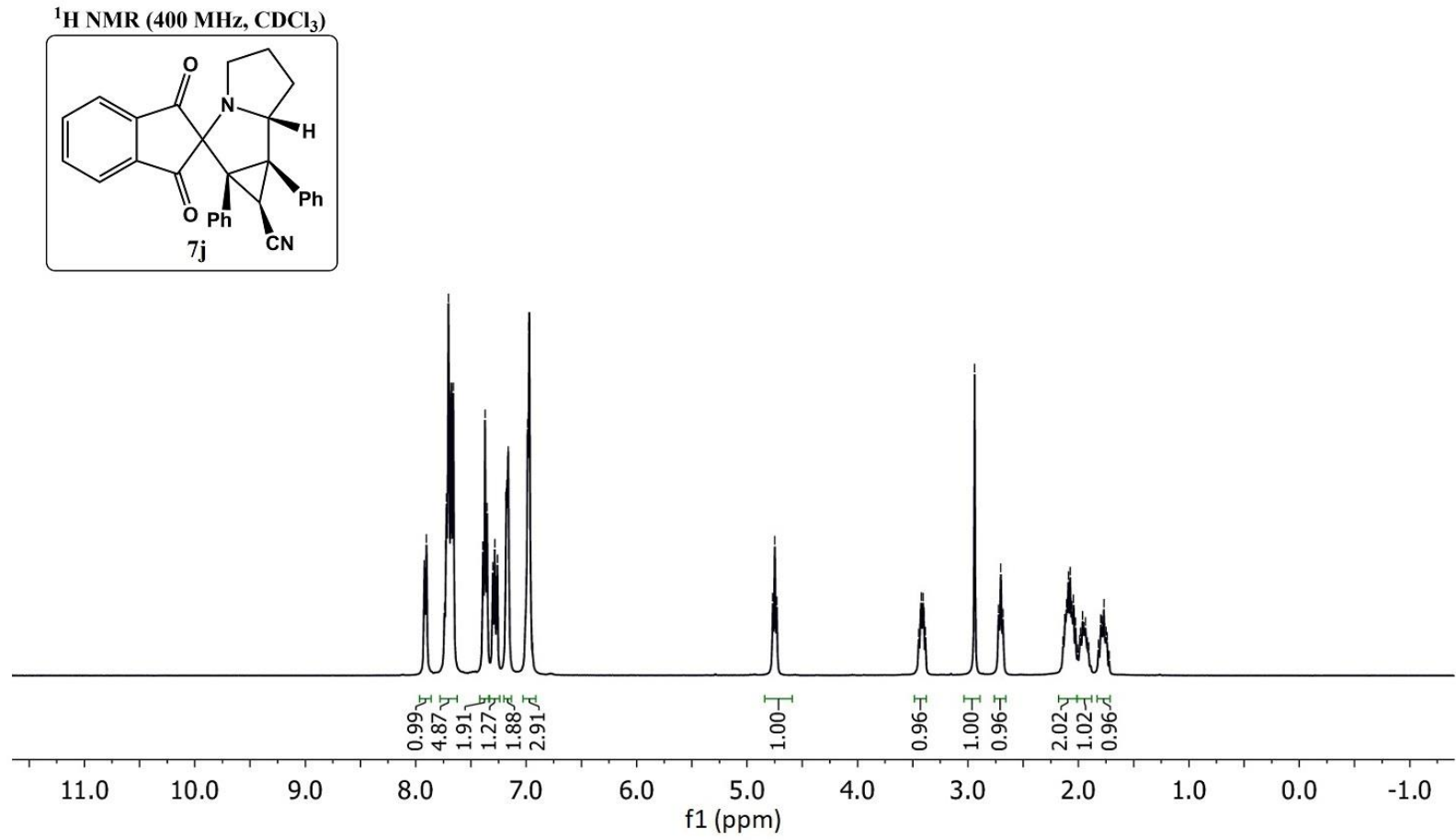

Figure S19. ${ }^{1} \mathrm{H}$ NMR spectrum of compound $7 \mathbf{j}\left(\mathrm{CDCl}_{3}, 400 \mathrm{MHz}\right)$
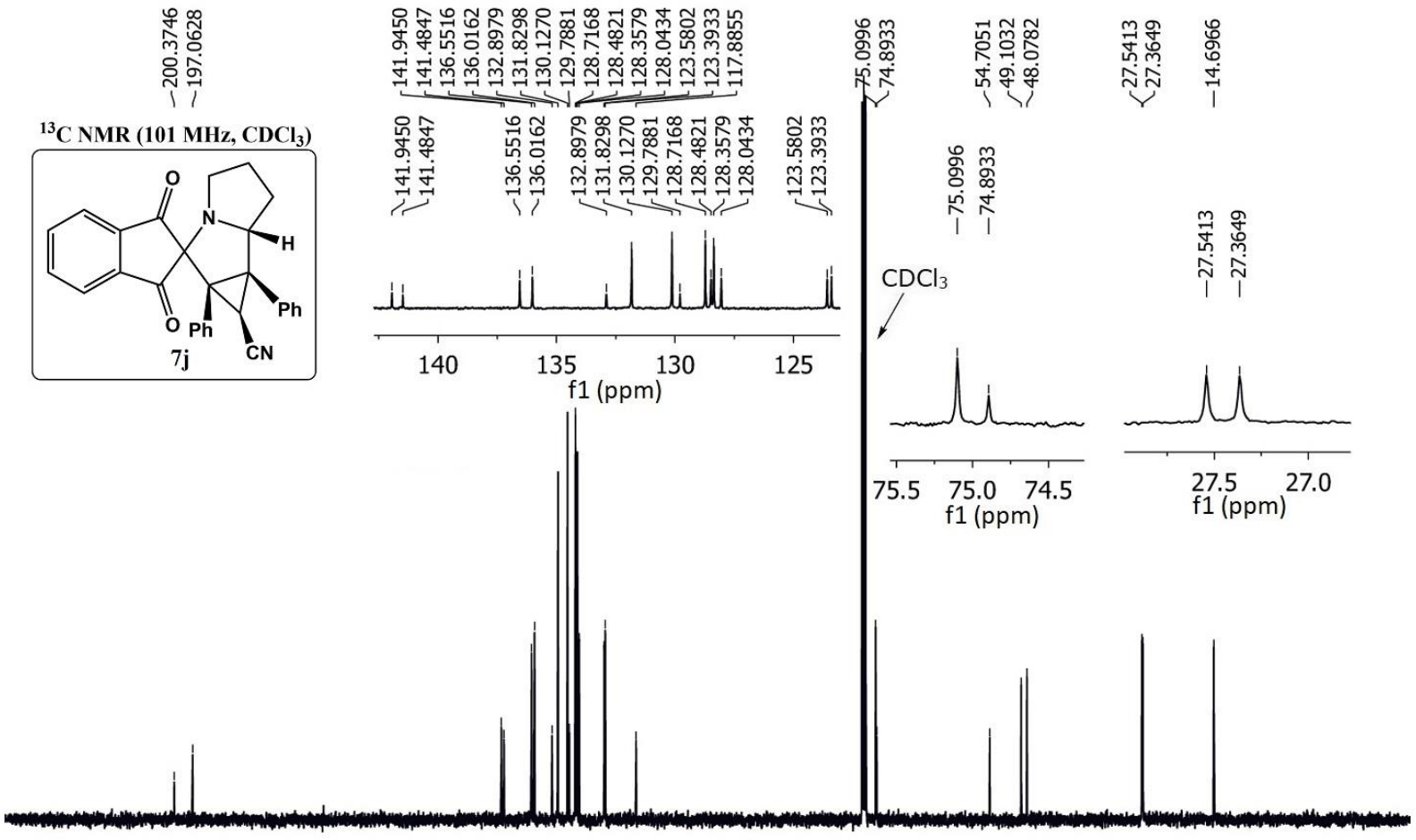

$\begin{array}{lllllllllllllllllllllll}220 & 210 & 200 & 190 & 180 & 170 & 160 & 150 & 140 & 130 & 120 & \begin{array}{c}110 \\ \mathrm{f} 1(\mathrm{ppm})\end{array} & 90 & 80 & 70 & 60 & 50 & 40 & 30 & 20 & 10 & 0 & -10\end{array}$

Figure S20. ${ }^{1} \mathrm{H}$ NMR spectrum of compound $\mathbf{7 j}\left(\mathrm{CDCl}_{3}, 101 \mathrm{MHz}\right)$ 
${ }^{1} \mathrm{H}$ NMR (400 MHz, $\left.\mathrm{CDCl}_{3}\right)$
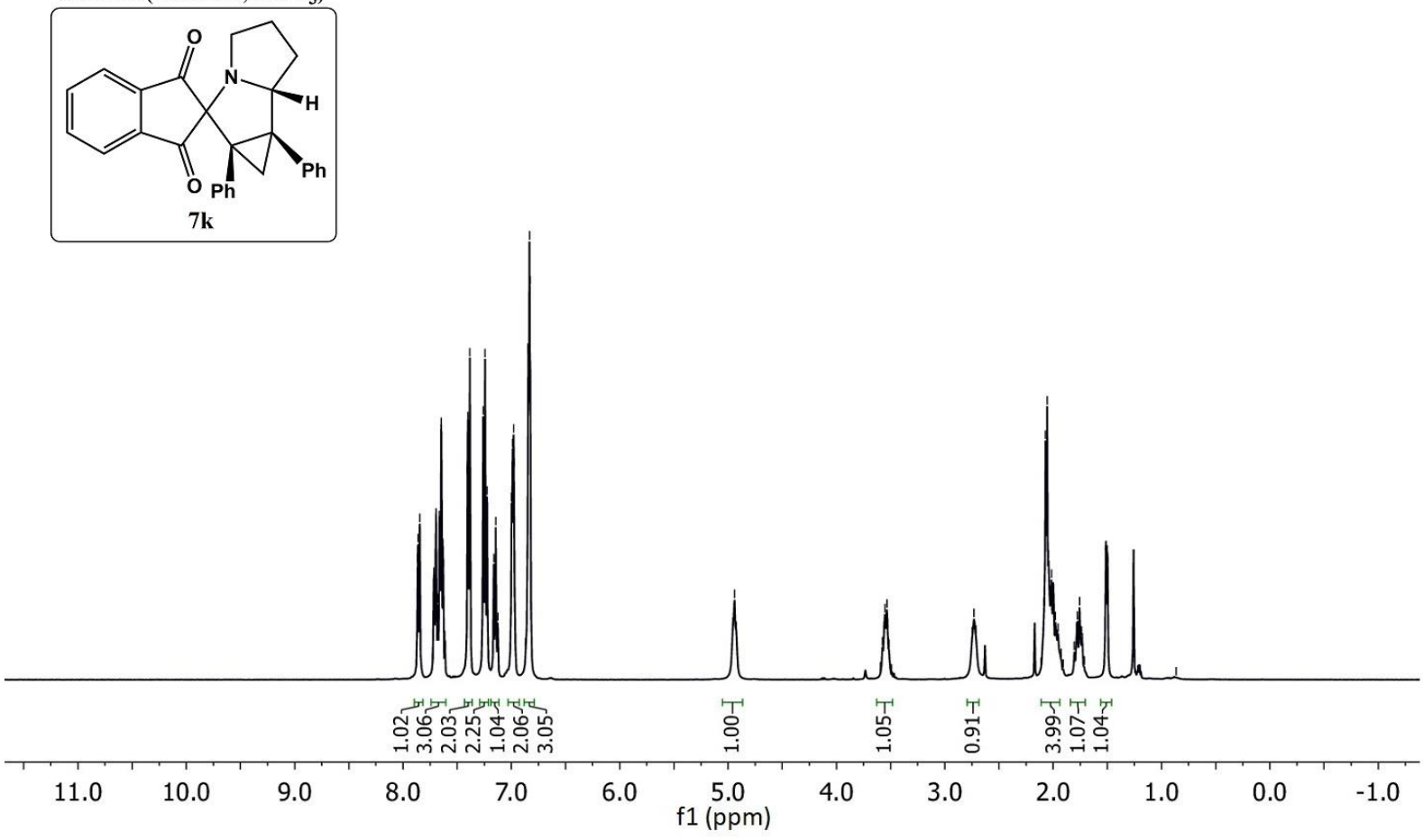

Figure S21. ${ }^{1} \mathrm{H}$ NMR spectrum of compound $7 \mathbf{k}\left(\mathrm{CDCl}_{3}, 400 \mathrm{MHz}\right)$

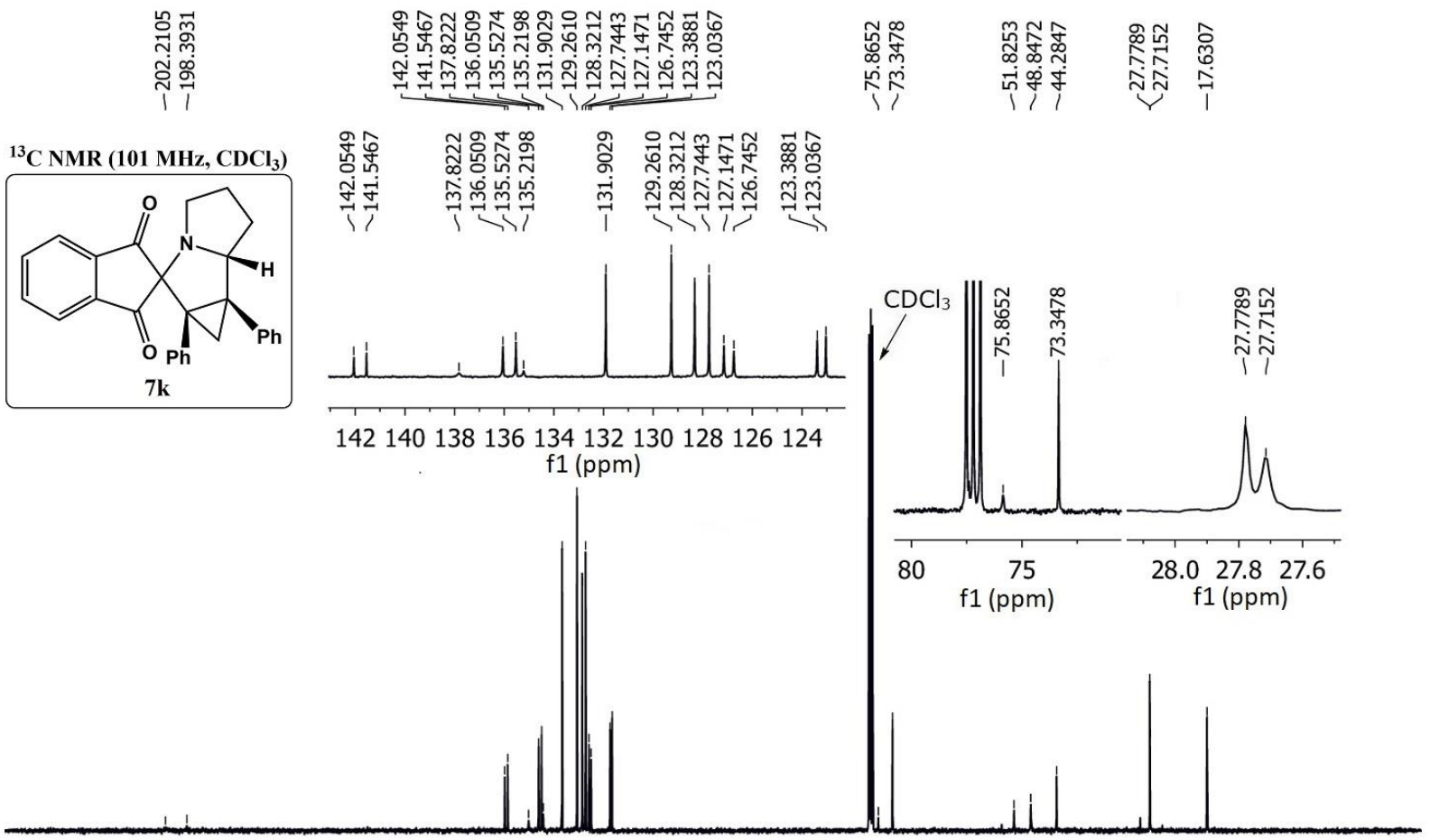

$\begin{array}{lllllllllllllllllllllll}220 & 210 & 200 & 190 & 180 & 170 & 160 & 150 & 140 & 130 & 120 & \begin{array}{l}110 \\ \mathrm{f} 1(\mathrm{ppm})\end{array} & 90 & 80 & 70 & 60 & 50 & 40 & 30 & 20 & 10 & 0 & -10\end{array}$

Figure S22. ${ }^{13} \mathrm{C}$ NMR spectrum of compound $7 \mathbf{k}\left(\mathrm{CDCl}_{3}, 101 \mathrm{MHz}\right)$ 


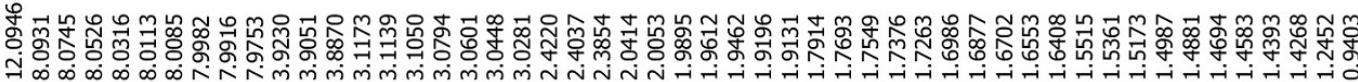

${ }^{1}$ H NMR (400 MHz, DMSO- $\left.d_{6}\right)$
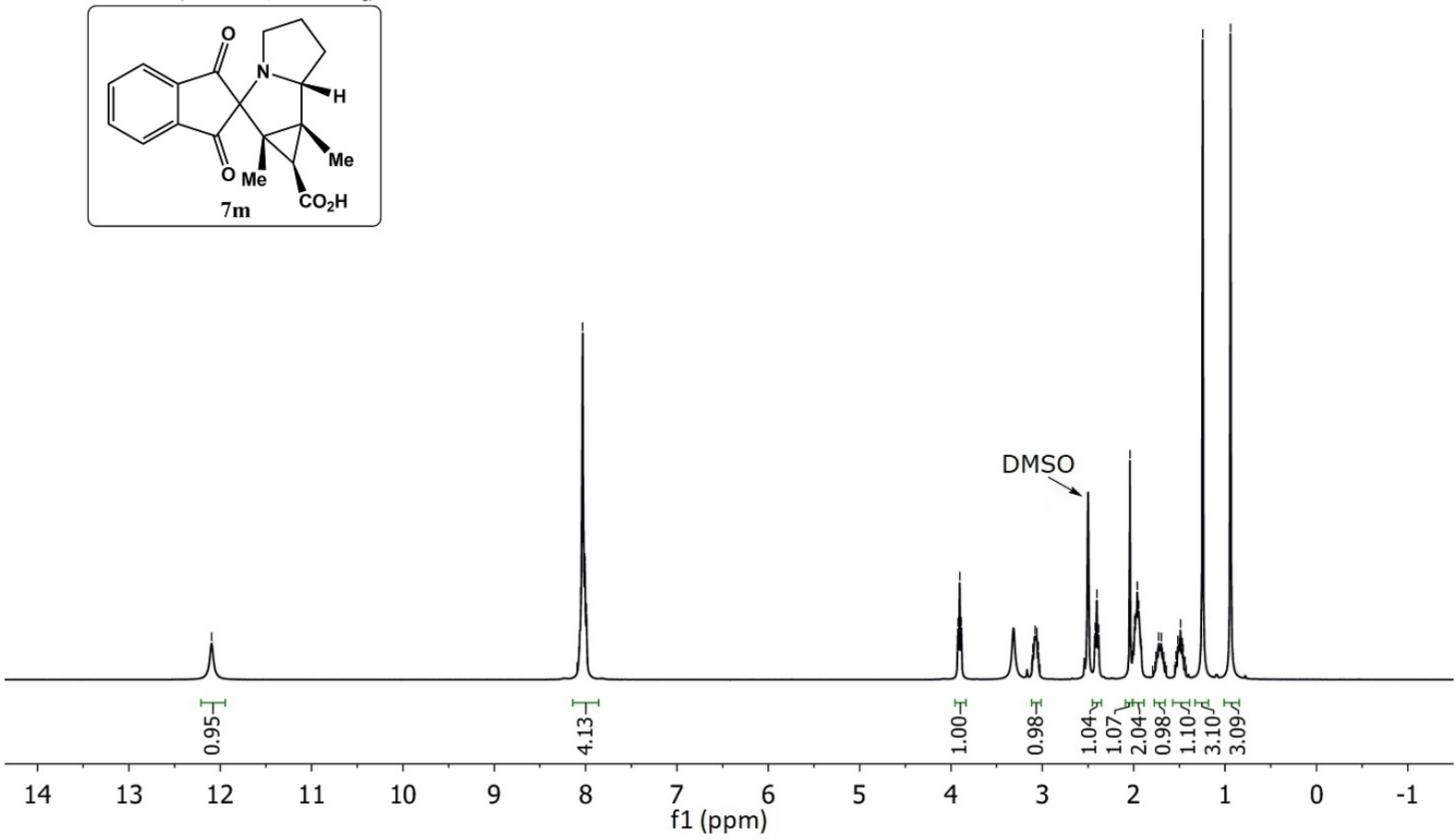

Figure S25. ${ }^{1} \mathrm{H}$ NMR spectrum of compound $\mathbf{7 m}\left(\mathrm{DMSO}-d_{6}, 400 \mathrm{MHz}\right)$

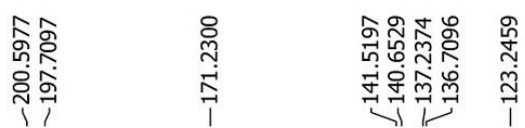
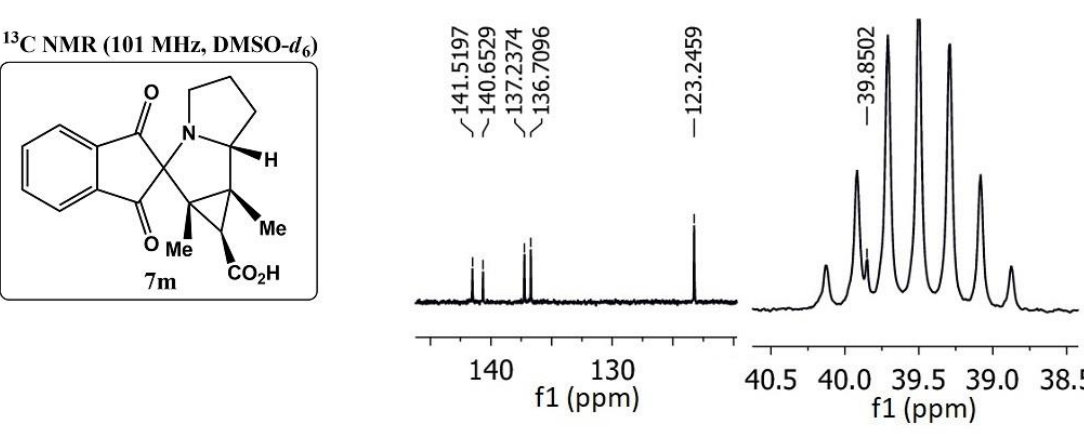

DMSO- $d_{6}$

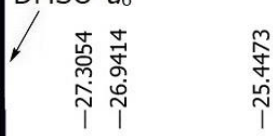
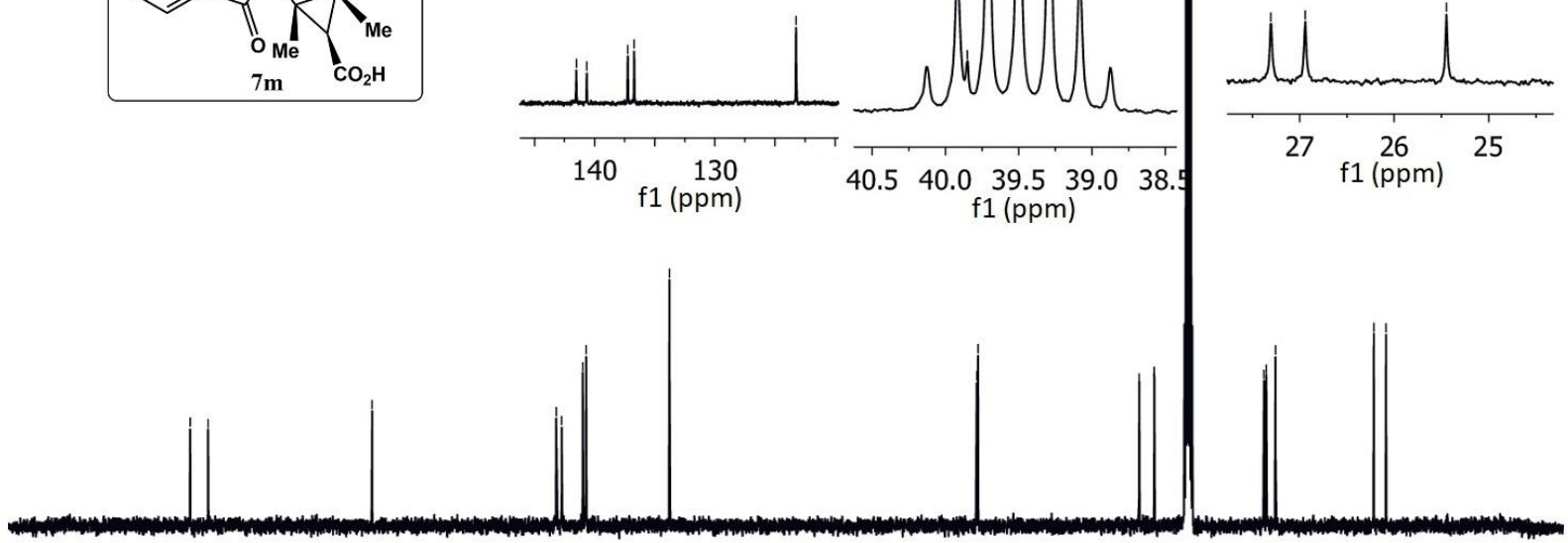

$\begin{array}{lllllllllllllllllllllll}220 & 210 & 200 & 190 & 180 & 170 & 160 & 150 & 140 & 130 & 120 & 110 \quad \begin{array}{l}100 \\ \mathrm{f} 1(\mathrm{ppm})\end{array} & 90 & 80 & 70 & 60 & 50 & 40 & 30 & 20 & 10 & 0 & -10\end{array}$

Figure S26. ${ }^{13} \mathrm{C}$ NMR spectrum of compound $7 \mathbf{m}\left(\mathrm{DMSO}-d_{6}, 101 \mathrm{MHz}\right)$ 


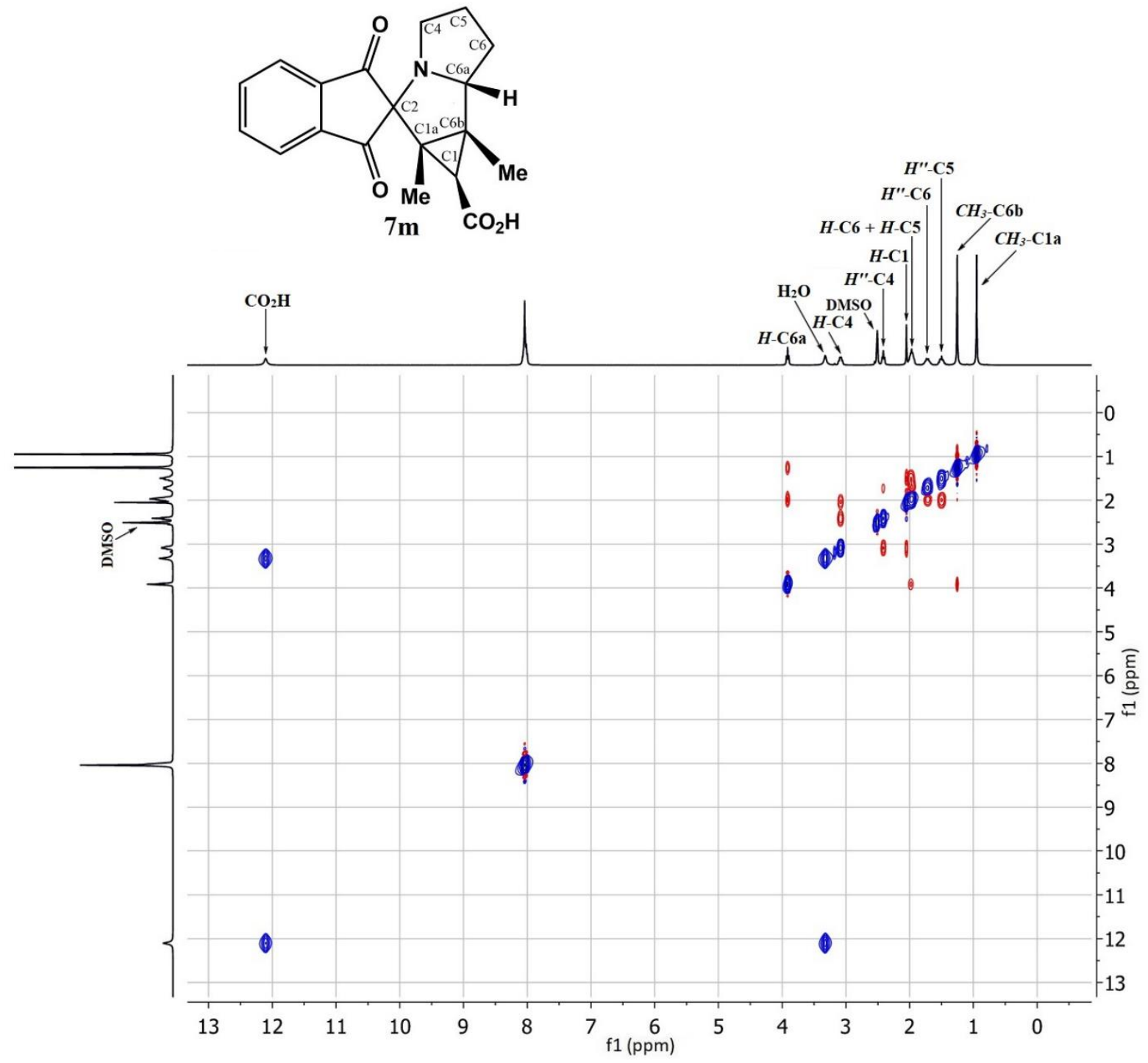

Figure S27. $2 \mathrm{D}{ }^{1} \mathrm{H}-{ }^{1} \mathrm{H}$ NOESY spectrum of compound 7m (DMSO- $\left.d_{6}, 400 \mathrm{MHz}\right)$ 

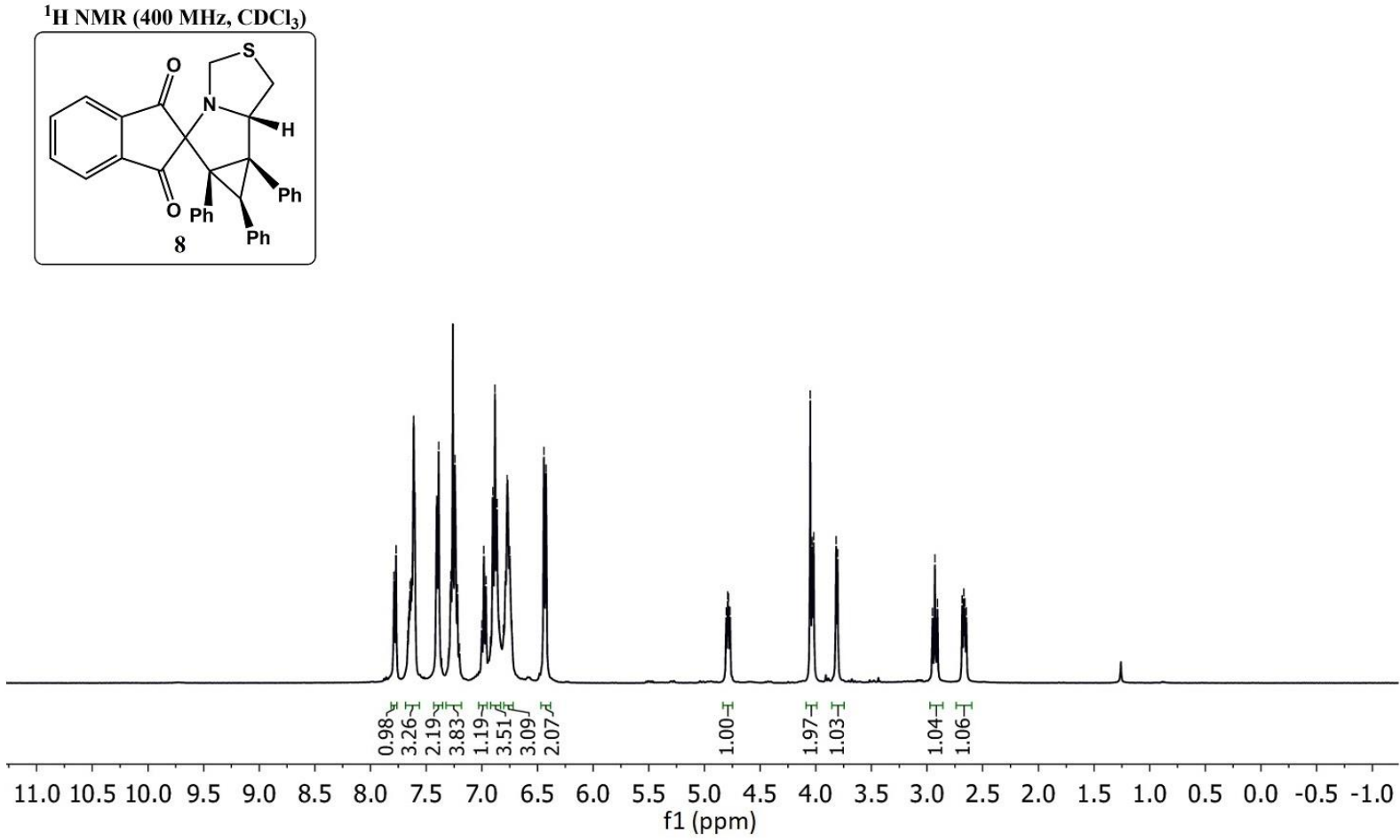

Figure S28. ${ }^{1} \mathrm{H}$ NMR spectrum of compound $\mathbf{8}\left(\mathrm{CDCl}_{3}, 400 \mathrm{MHz}\right)$
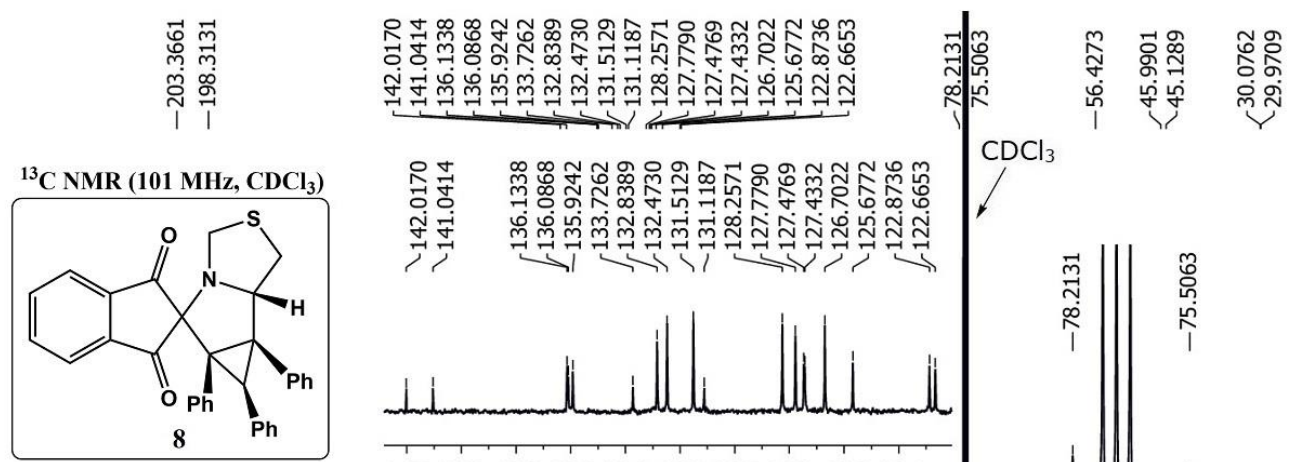

$\mathrm{CDCl}_{3}$

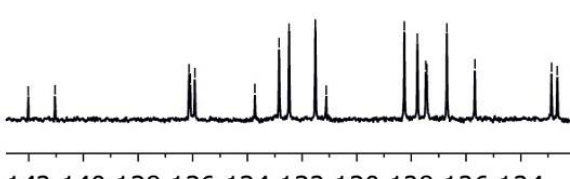

142140138136134132130128126124 f1 (ppm)
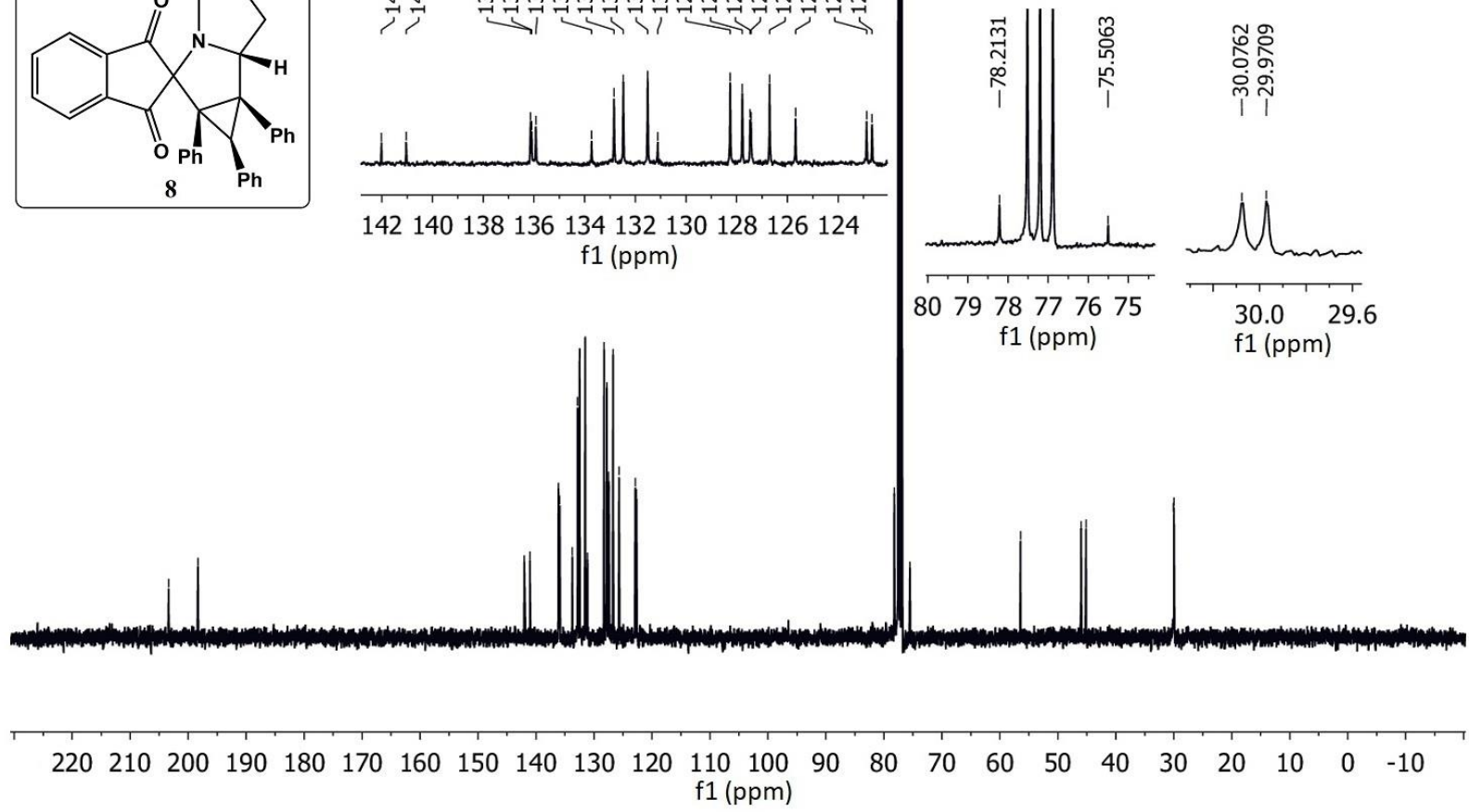

Figure S29. ${ }^{13} \mathrm{C}$ NMR spectrum of compound $8\left(\mathrm{CDCl}_{3}, 101 \mathrm{MHz}\right)$ 


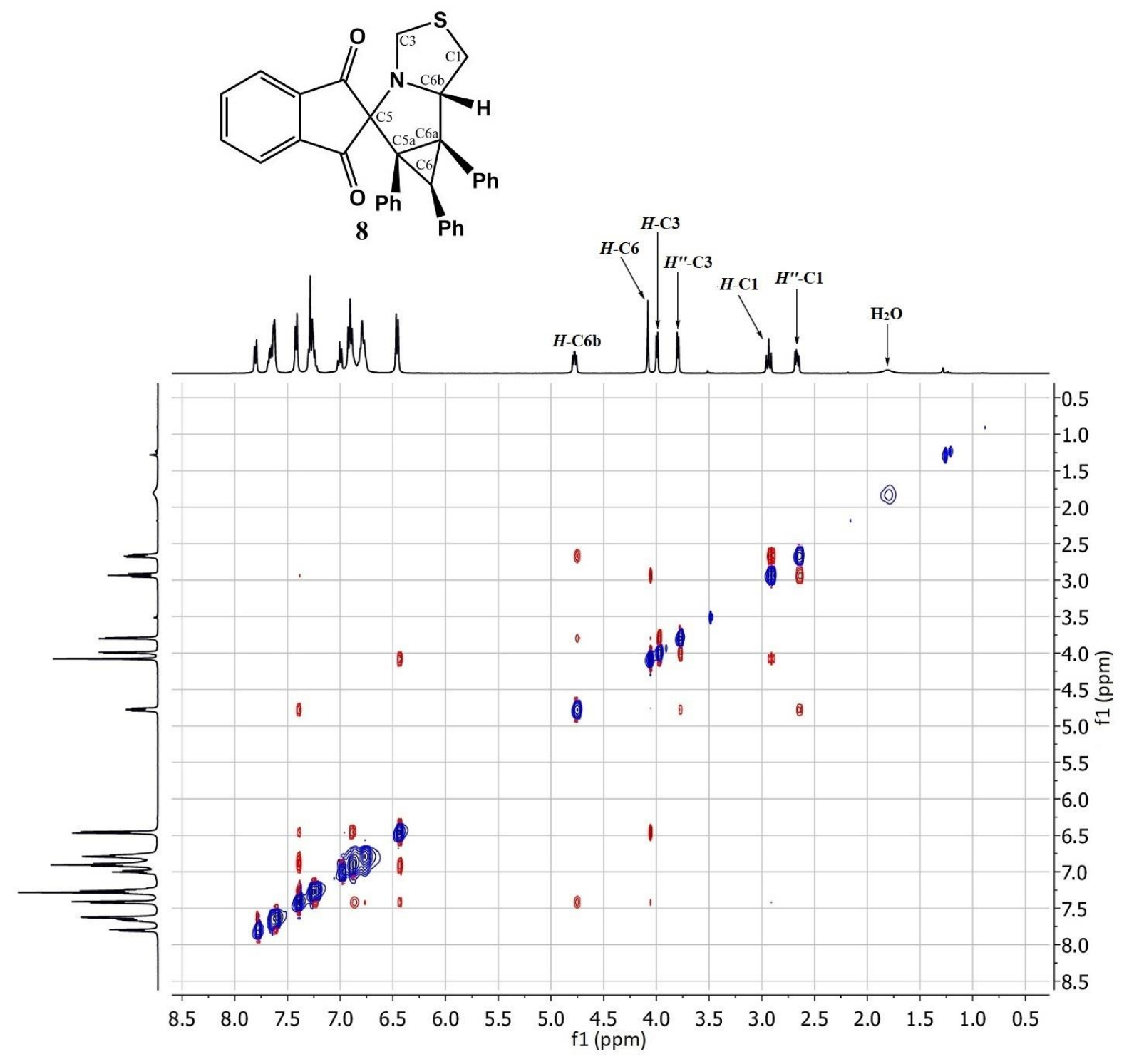

Figure S30. 2D ${ }^{1} \mathrm{H}-{ }^{1} \mathrm{H}$ NOESY spectrum of compound $8\left(\mathrm{CDCl}_{3}, 400 \mathrm{MHz}\right)$ 

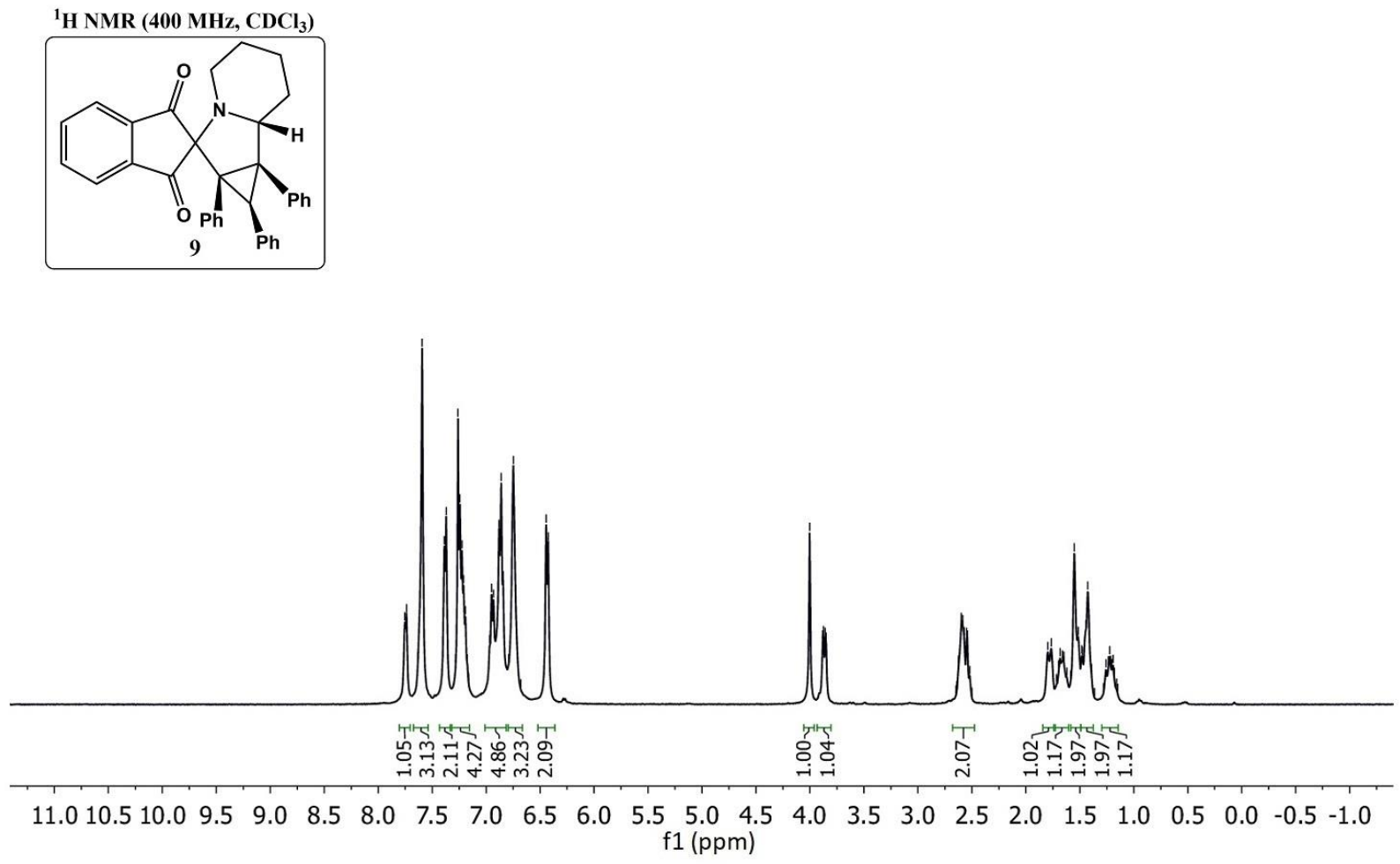

Figure S31. ${ }^{1} \mathrm{H}$ NMR spectrum of compound $9\left(\mathrm{CDCl}_{3}, 400 \mathrm{MHz}\right)$

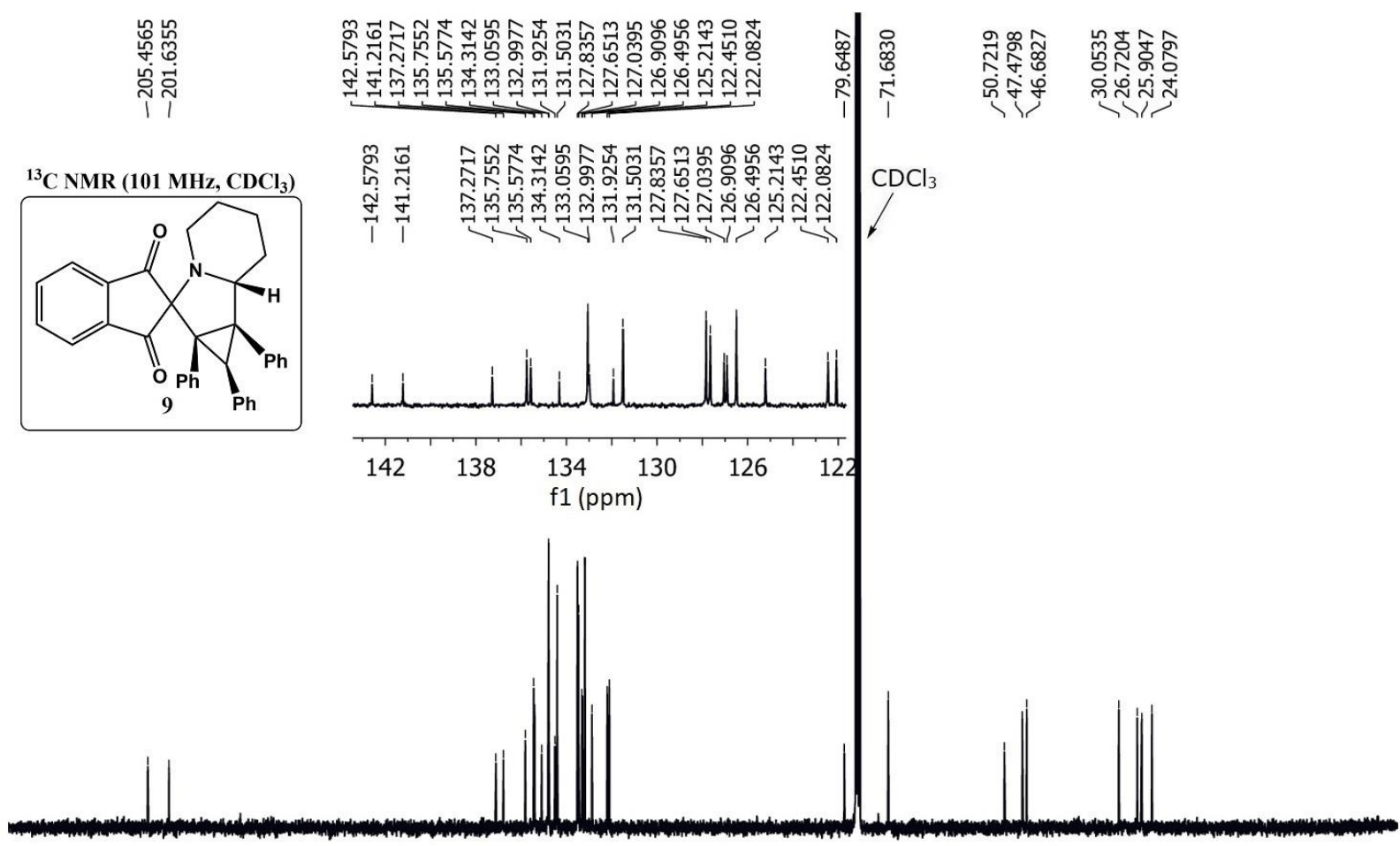

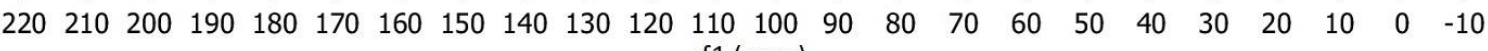
f1 (ppm)

Figure S32. ${ }^{13} \mathrm{C}$ NMR spectrum of compound $9\left(\mathrm{CDCl}_{3}, 101 \mathrm{MHz}\right)$ 


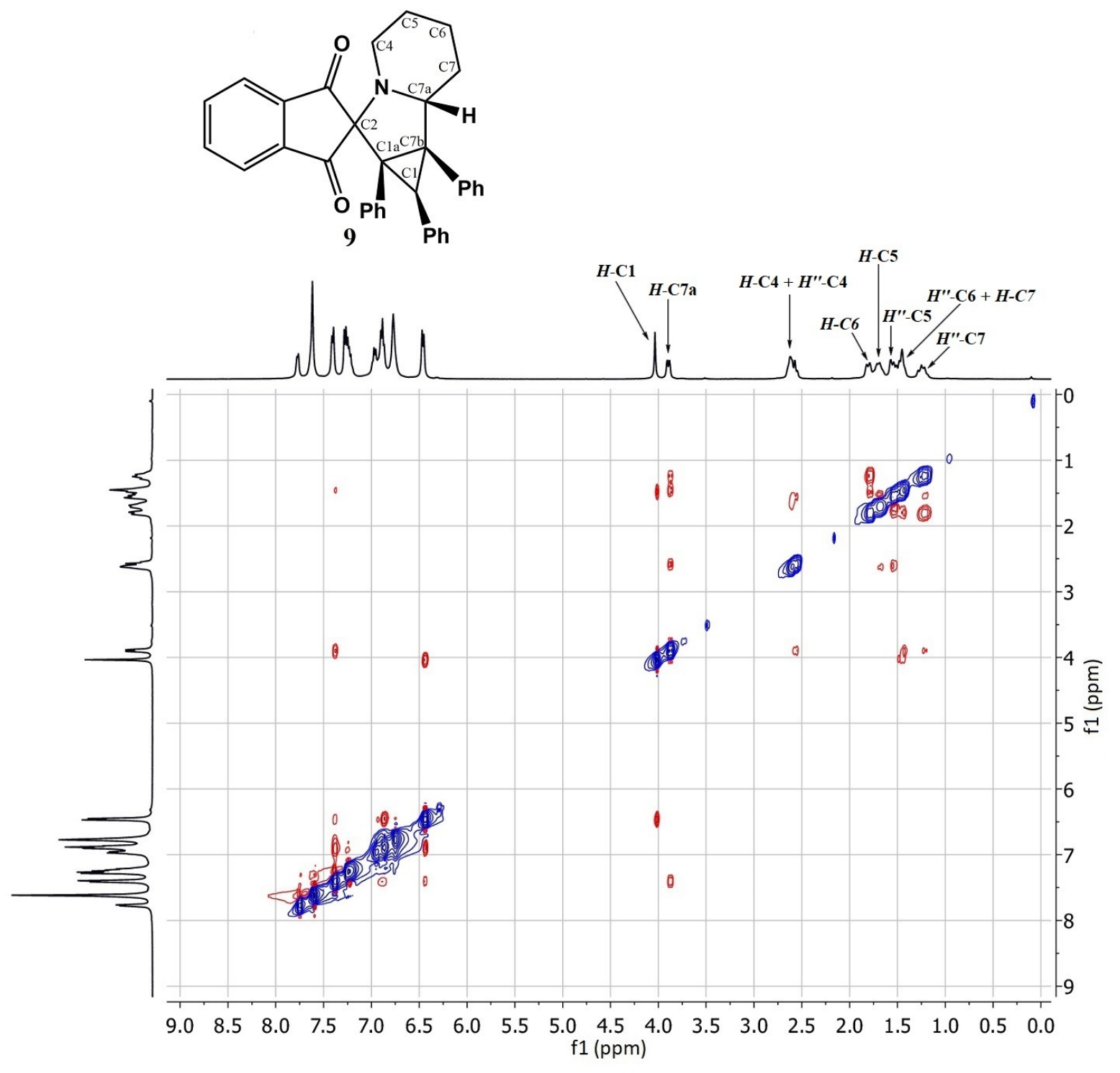

Figure S33. 2D ${ }^{1} \mathrm{H}-{ }^{1} \mathrm{H}$ NOESY spectrum of compound $9\left(\mathrm{CDCl}_{3}, 400 \mathrm{MHz}\right)$ 
${ }^{1} \mathrm{H}$ NMR (400 MHz, $\left.\mathrm{CDCl}_{3}\right)$
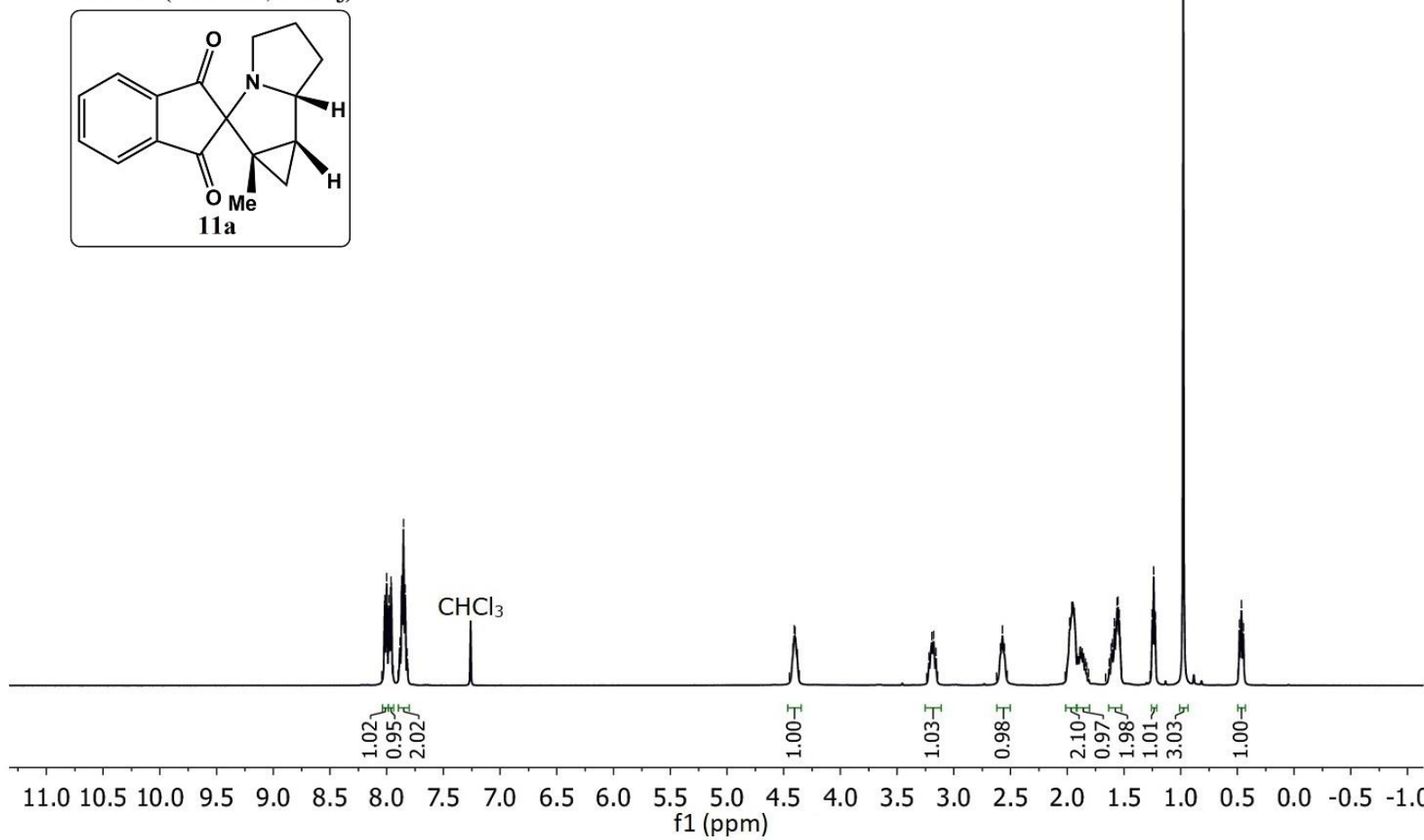

Figure S34. ${ }^{1} \mathrm{H}$ NMR spectrum of compound $11 a\left(\mathrm{CDCl}_{3}, 400 \mathrm{MHz}\right)$
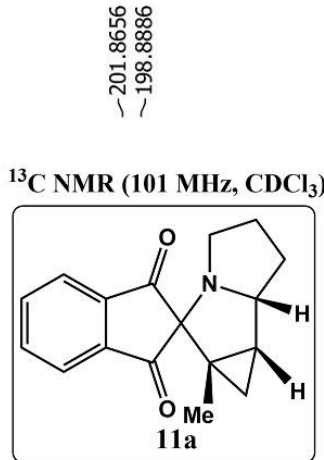
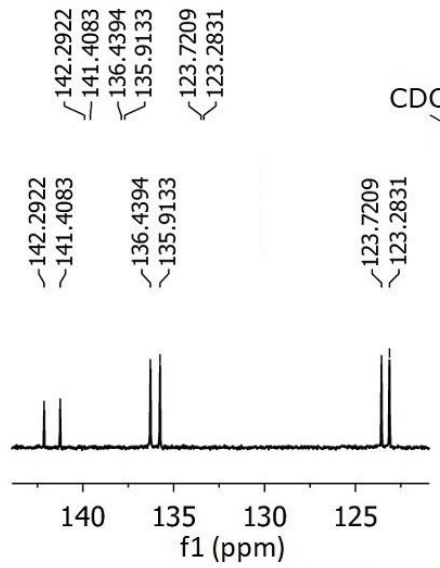

㤅

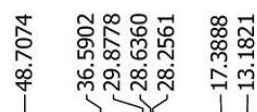

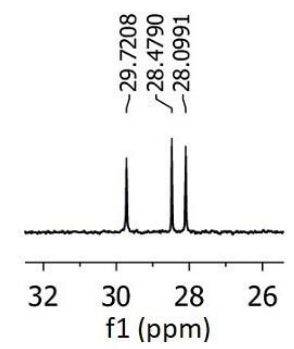

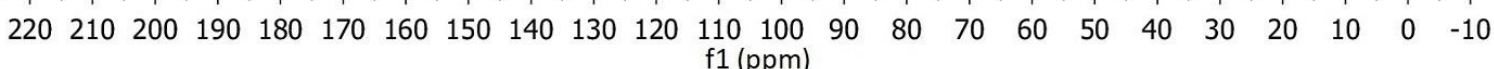

Figure S35. ${ }^{13} \mathrm{C}$ NMR spectrum of compound $11 a\left(\mathrm{CDCl}_{3}, 101 \mathrm{MHz}\right)$ 


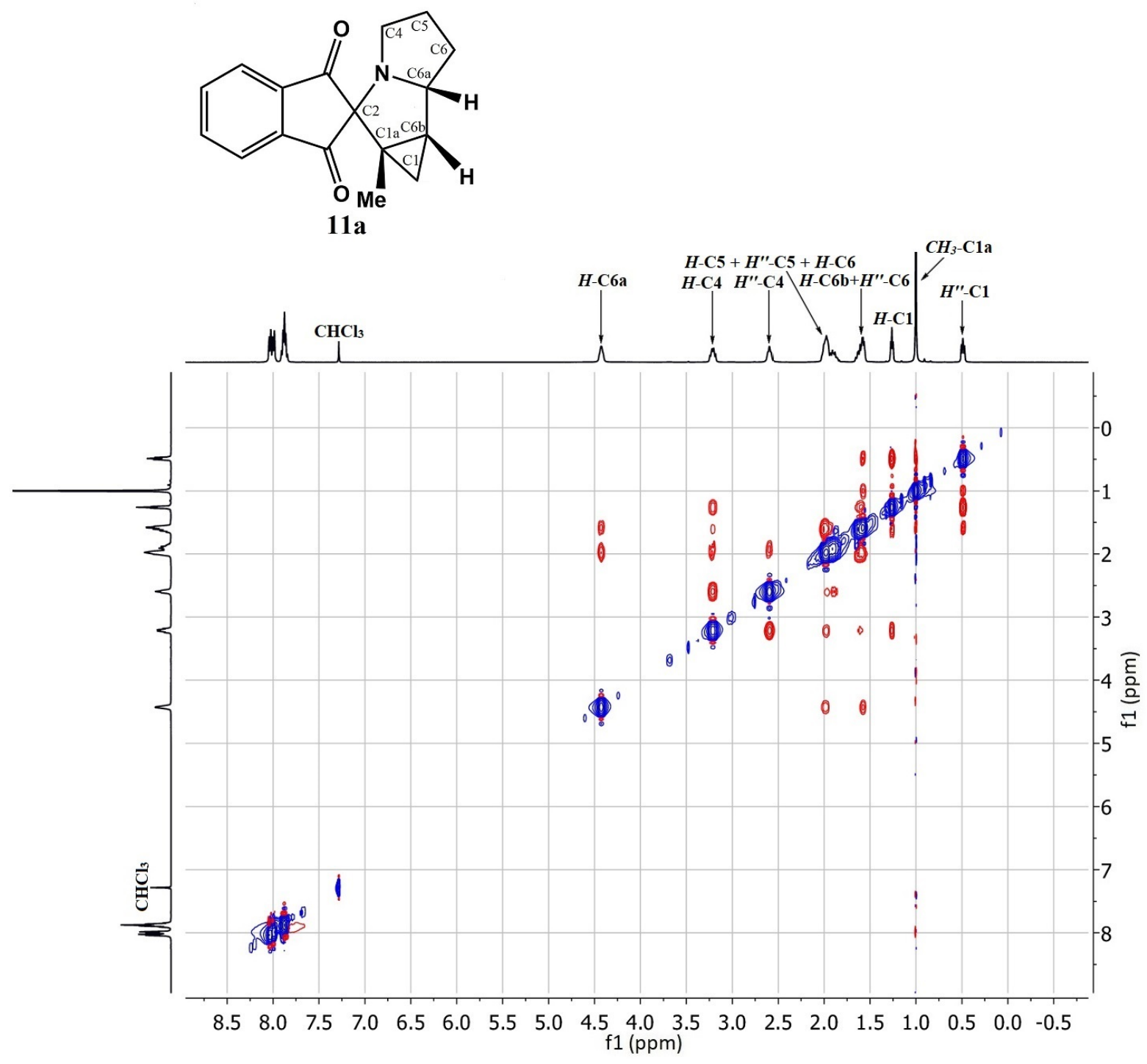

Figure S36. 2D ${ }^{1} \mathrm{H}-{ }^{1} \mathrm{H}$ NOESY spectrum of compound $11 \mathrm{a}\left(\mathrm{CDCl}_{3}, 400 \mathrm{MHz}\right)$ 


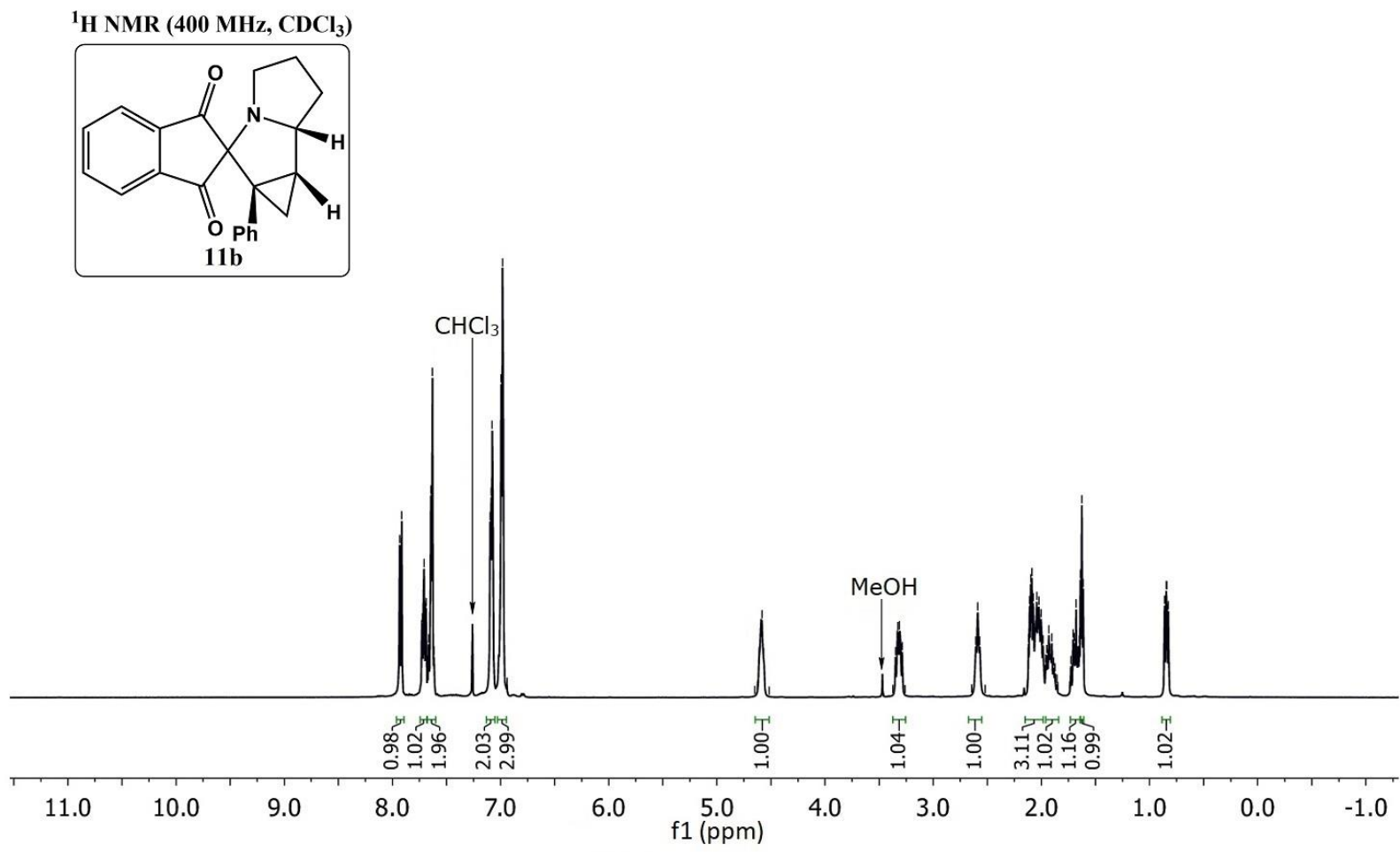

Figure S37. ${ }^{1} \mathrm{H}$ NMR spectrum of compound $\mathbf{1 1 b}\left(\mathrm{CDCl}_{3}, 400 \mathrm{MHz}\right)$

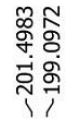

${ }^{13} \mathrm{C}$ NMR (101 MHz, $\left.\mathrm{CDCl}_{3}\right)$

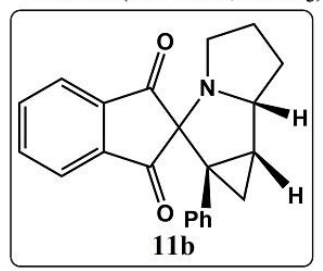

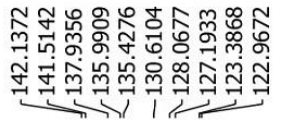
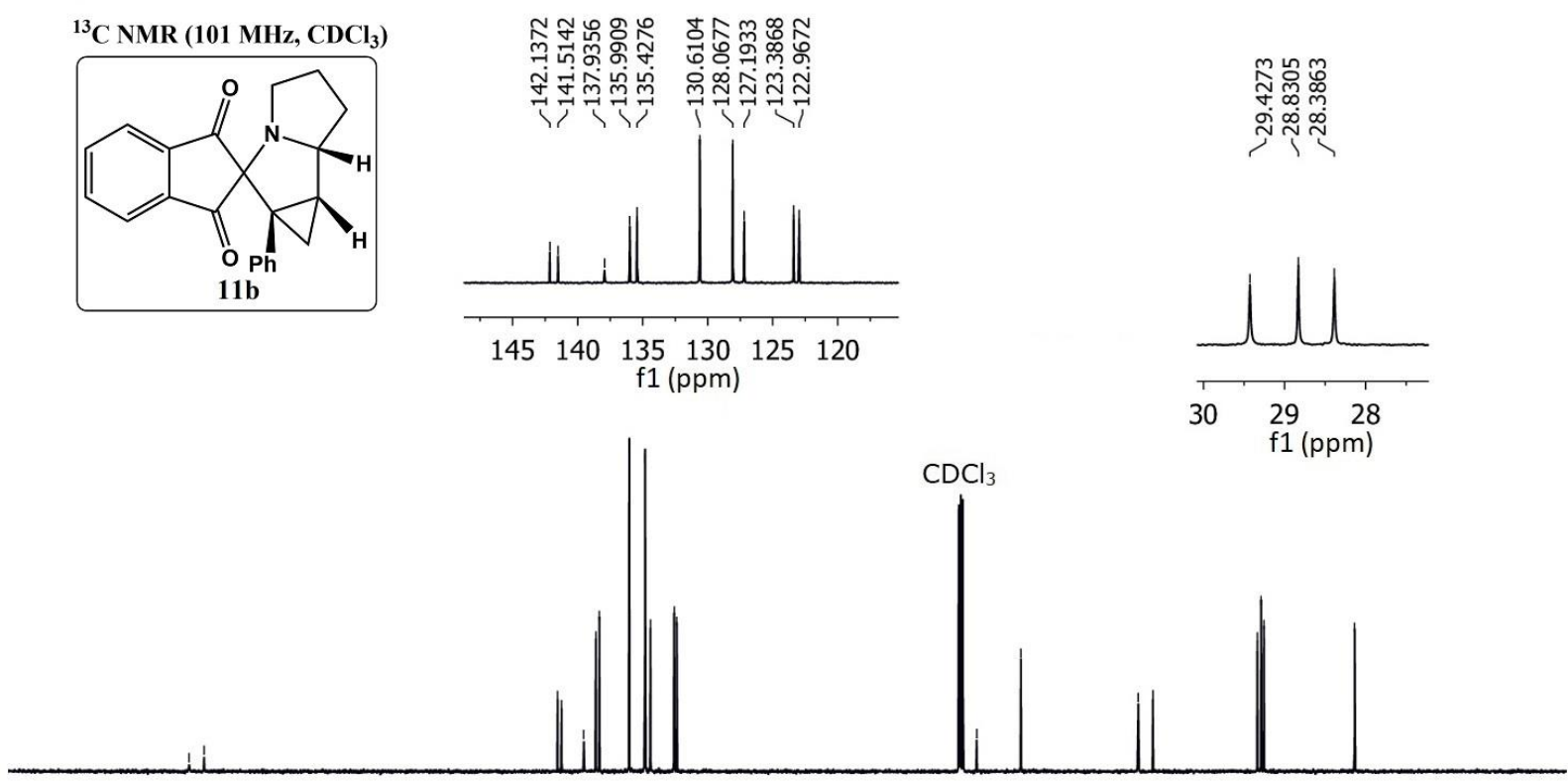

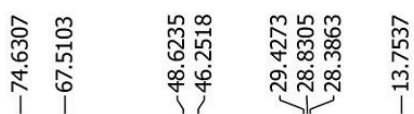

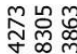

จั

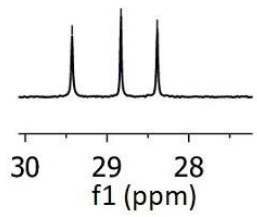

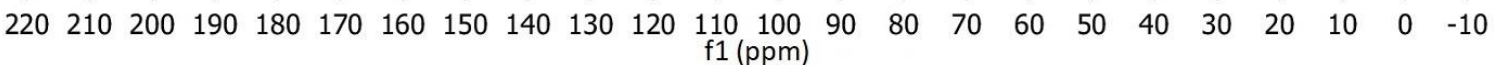

Figure S38. ${ }^{13} \mathrm{C}$ NMR spectrum of compound $\mathbf{1 1 b}\left(\mathrm{CDCl}_{3}, 101 \mathrm{MHz}\right)$ 


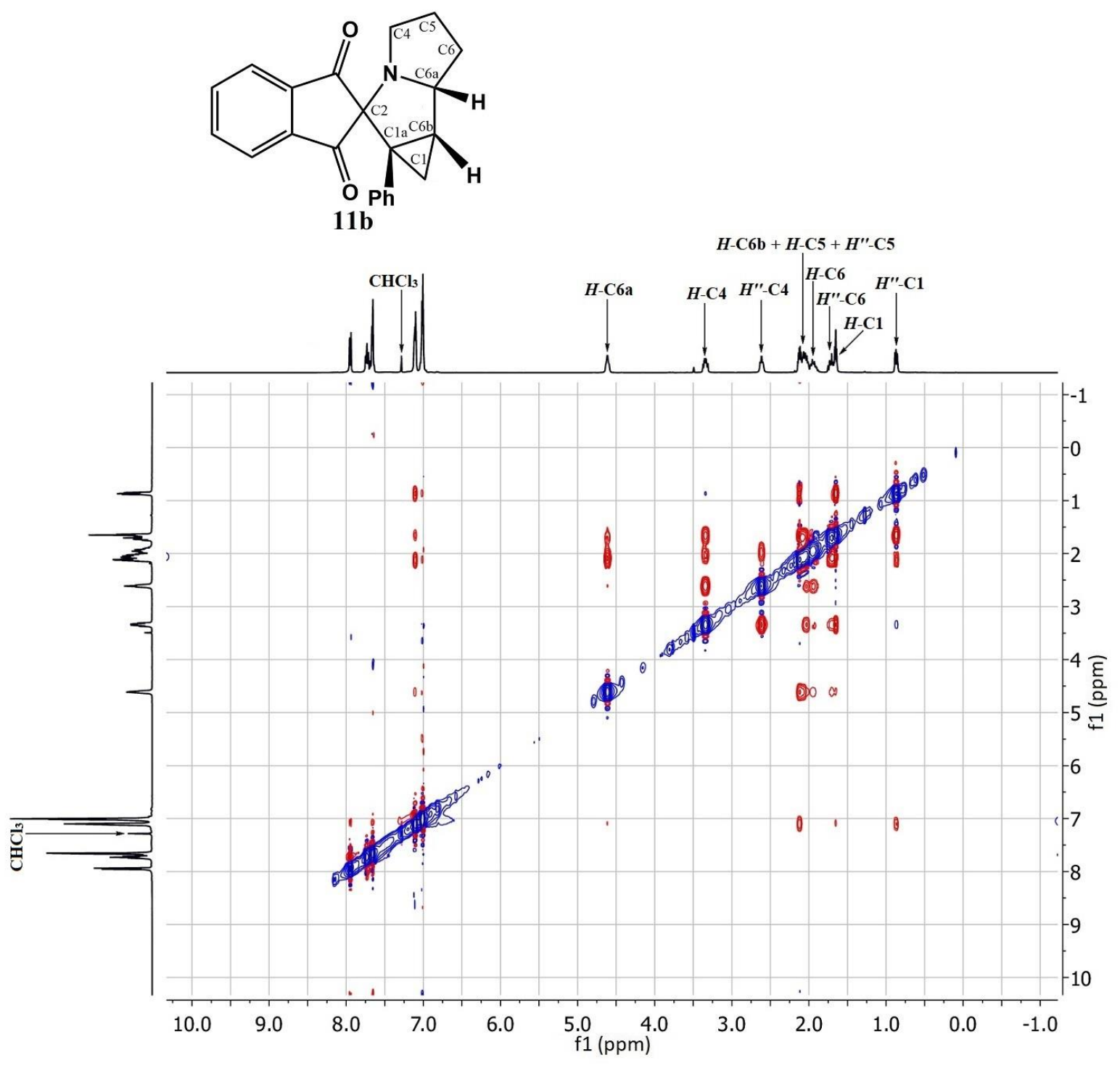

Figure S39. 2D ${ }^{1} \mathrm{H}-{ }^{1} \mathrm{H}$ NOESY spectrum of compound $\mathbf{1 1 b}\left(\mathrm{CDCl}_{3}, 400 \mathrm{MHz}\right)$ 
${ }^{1} \mathrm{H}$ NMR (400 MHz, $\left.\mathrm{CDCl}_{3}\right)$
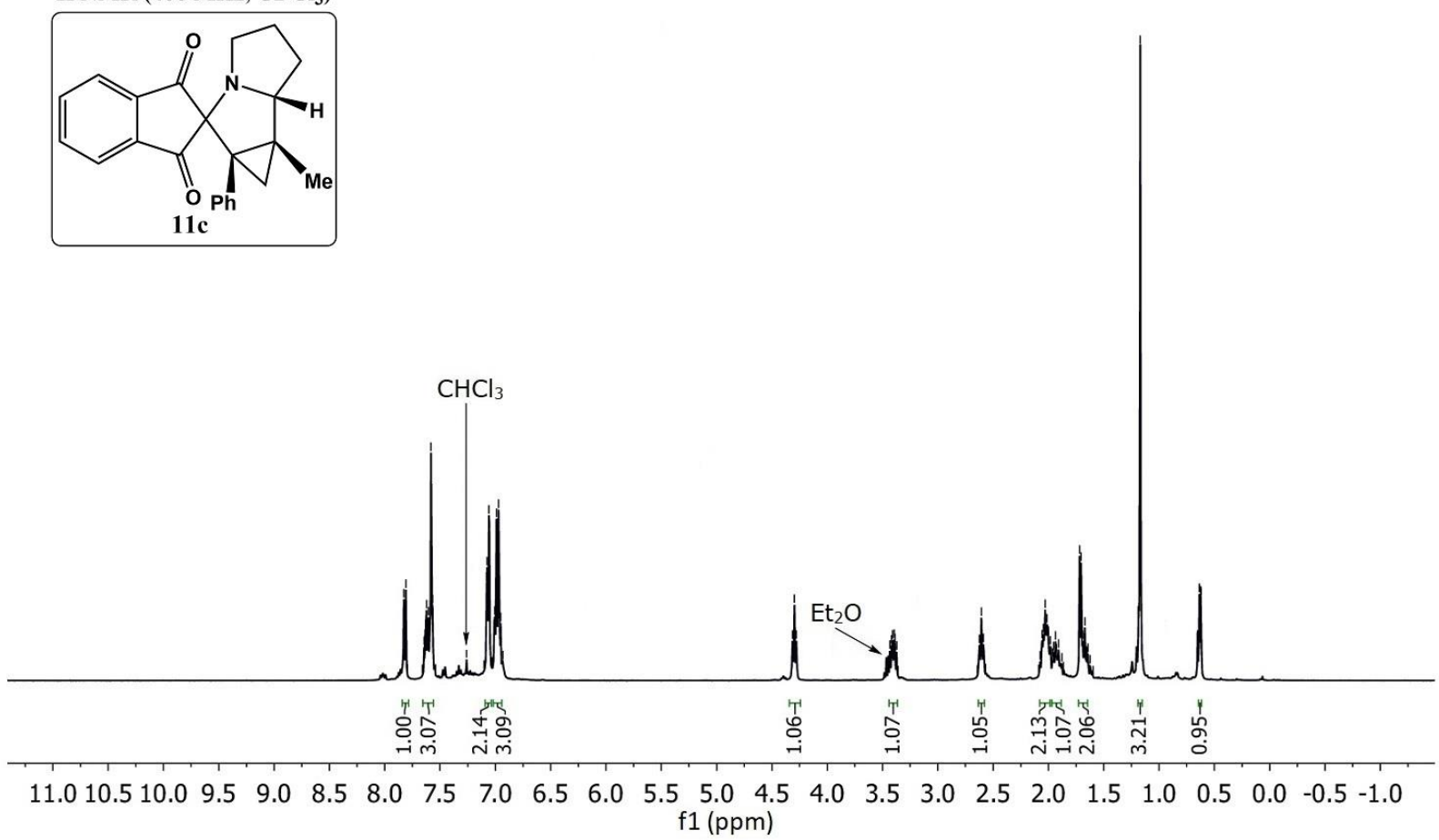

Figure S40. ${ }^{1} \mathrm{H} \mathrm{NMR}$ spectrum of compound $11 \mathrm{c}\left(\mathrm{CDCl}_{3}, 400 \mathrm{MHz}\right)$

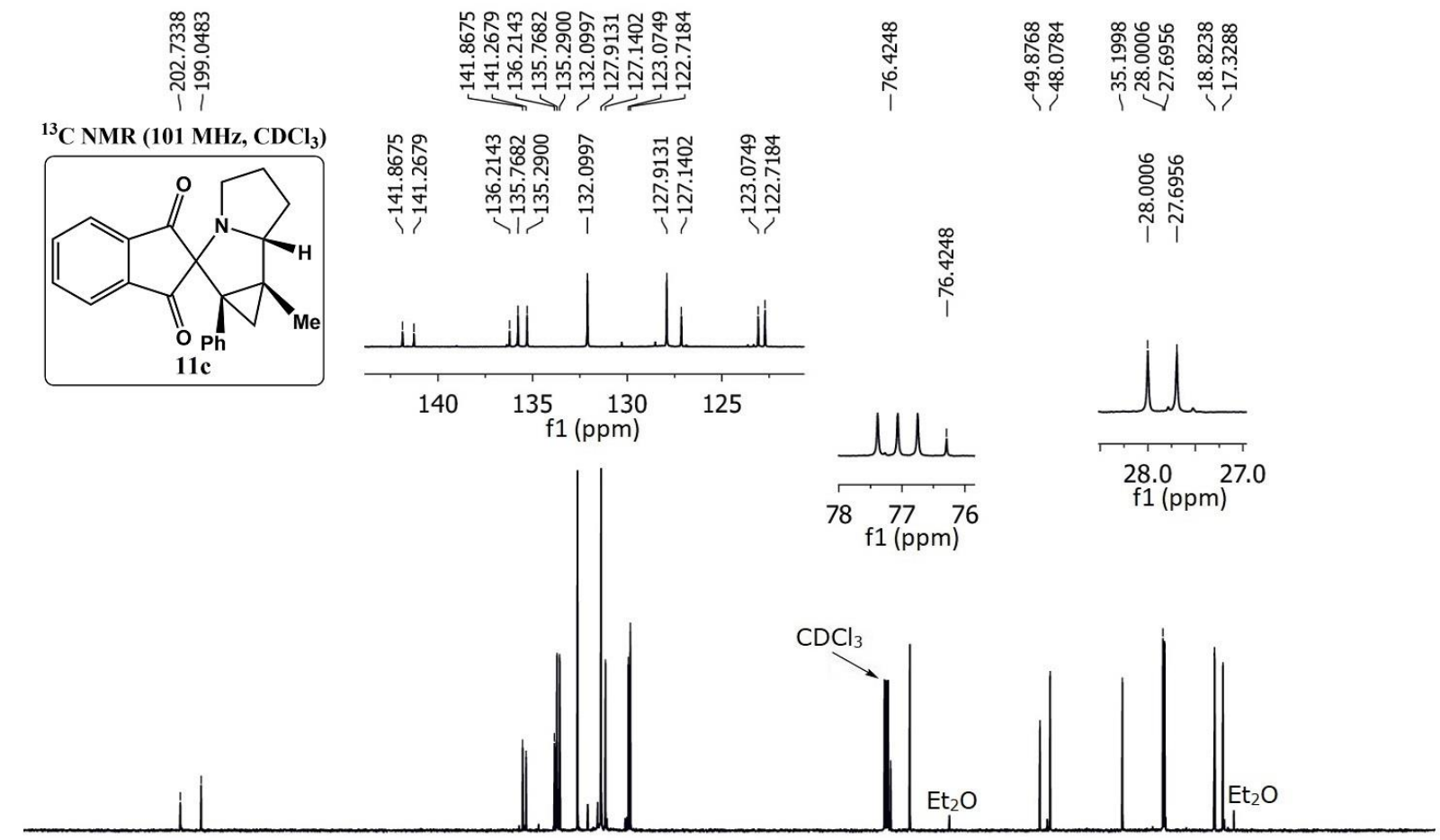

$\begin{array}{lllllllllllllllllllllll}220 & 210 & 200 & 190 & 180 & 170 & 160 & 150 & 140 & 130 & 120 & \begin{array}{l}110 \\ \mathrm{f} 1(\mathrm{ppm})\end{array} & 100 & 90 & 70 & 60 & 50 & 40 & 30 & 20 & 10 & 0 & -10\end{array}$

Figure S41. ${ }^{13} \mathrm{C}$ NMR spectrum of compound 11c $\left(\mathrm{CDCl}_{3}, 101 \mathrm{MHz}\right)$ 


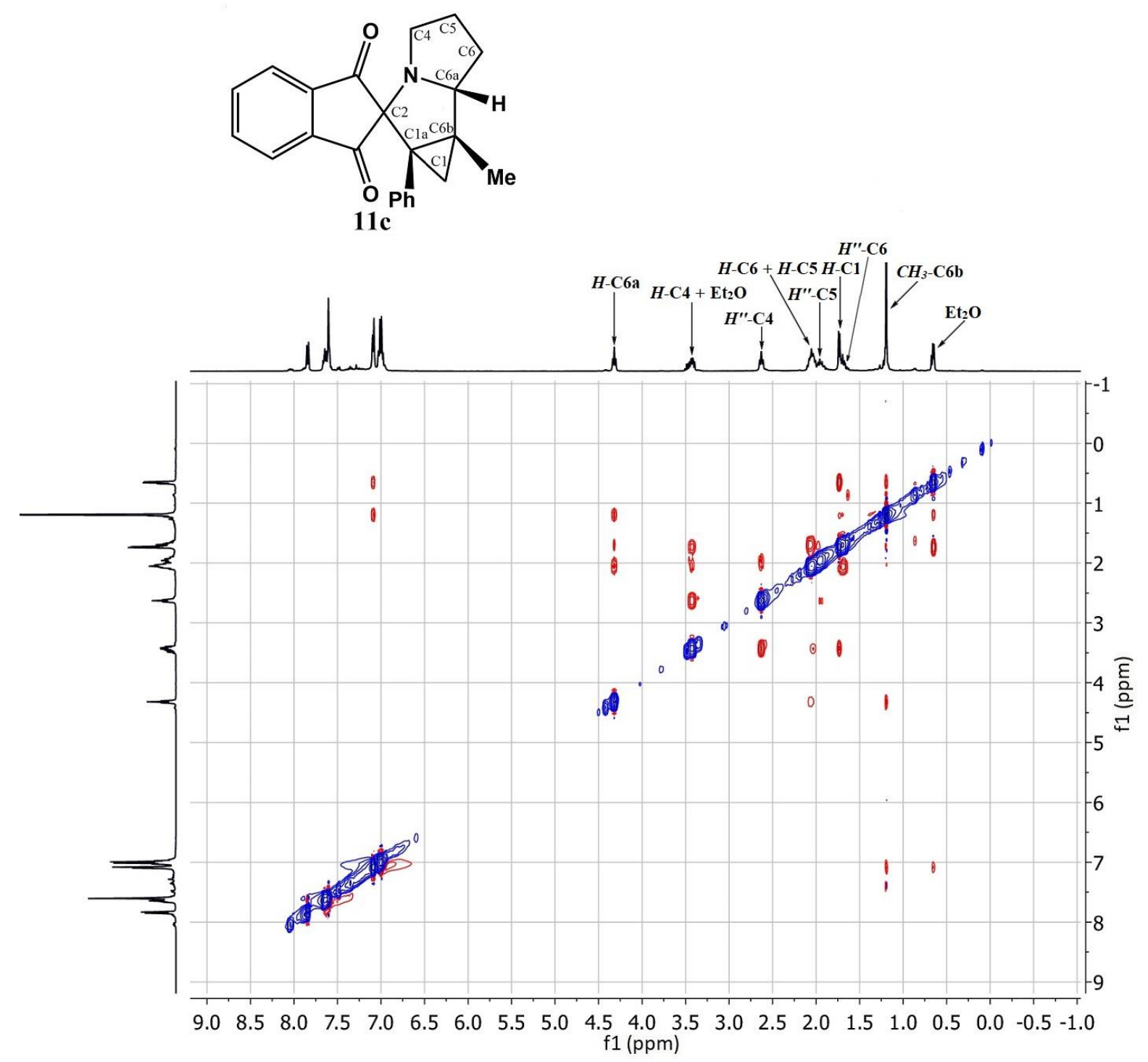

Figure S42. 2D ${ }^{1} \mathrm{H}-{ }^{1} \mathrm{H}$ NOESY spectrum of compound $11 \mathrm{c}\left(\mathrm{CDCl}_{3}, 400 \mathrm{MHz}\right)$ 


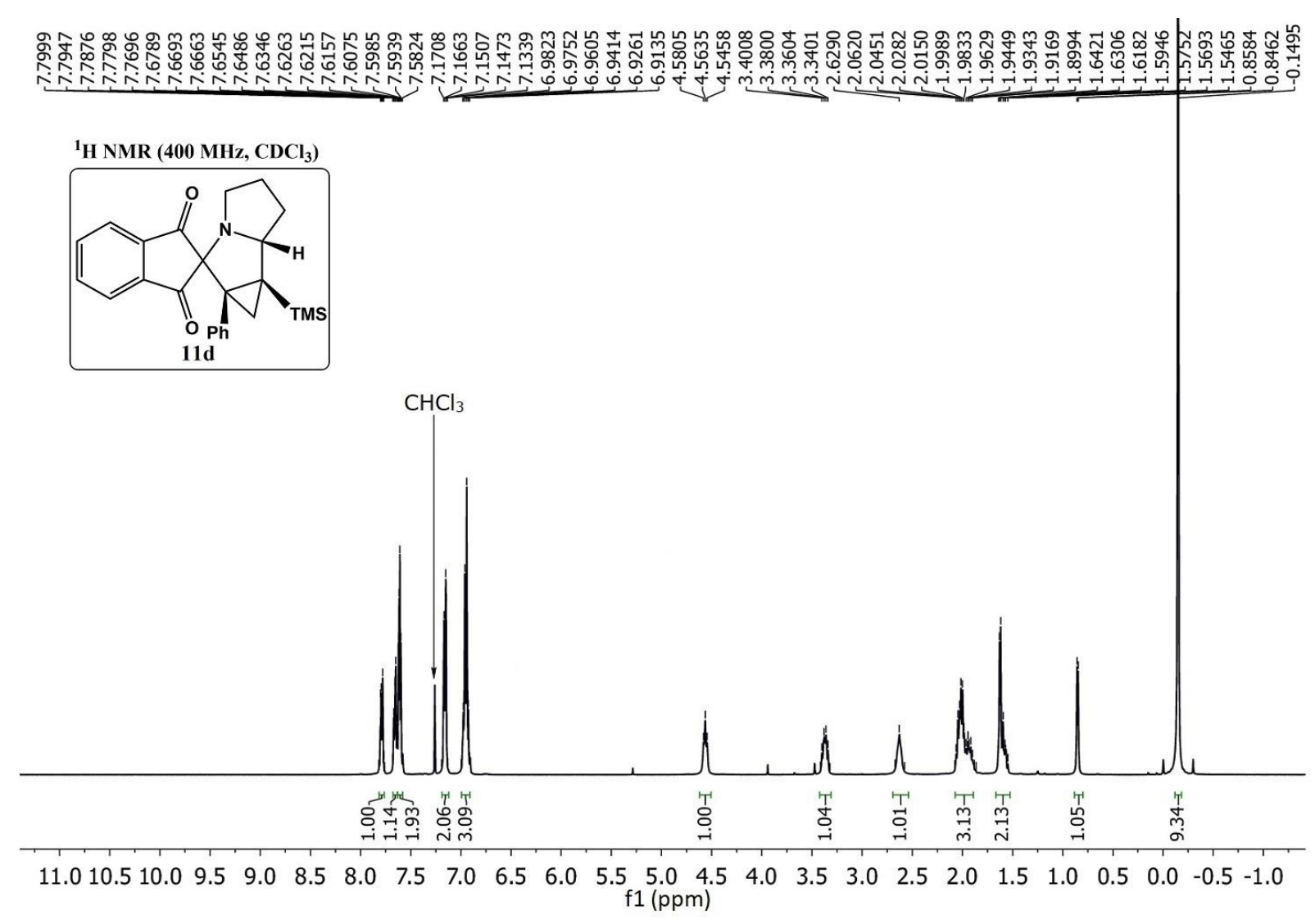

Figure S43. ${ }^{1} \mathrm{H}$ NMR spectrum of compound $11 d\left(\mathrm{CDCl}_{3}, 400 \mathrm{MHz}\right)$

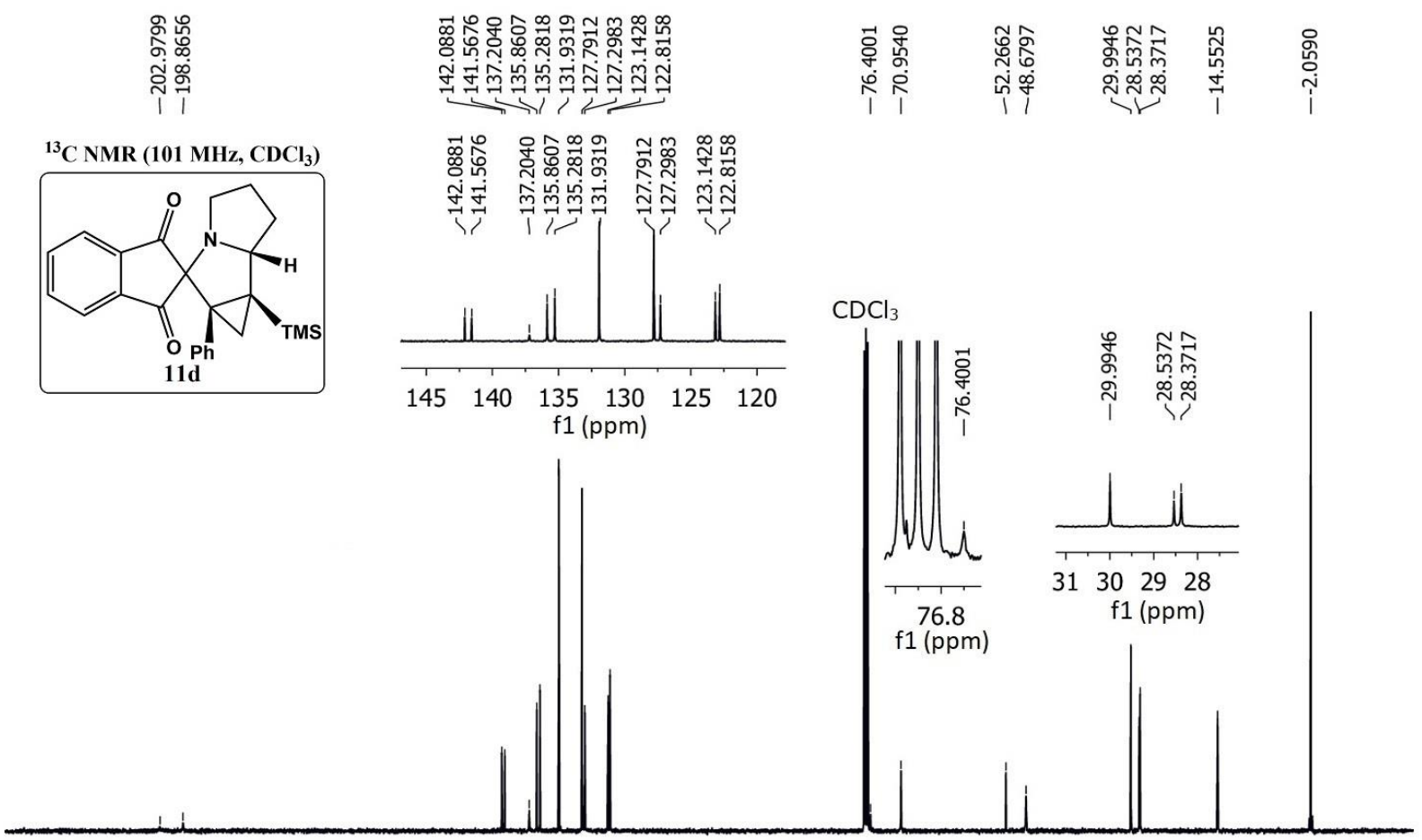

$\begin{array}{llllllllllllllllllllllll}220 & 210 & 200 & 190 & 180 & 170 & 160 & 150 & 140 & 130 & 120 & \begin{array}{l}110 \\ \mathrm{f} 1(\mathrm{ppm})\end{array} & 100 & 80 & 70 & 60 & 50 & 40 & 30 & 20 & 10 & 0 & -10\end{array}$

Figure S44. ${ }^{13} \mathrm{C}$ NMR spectrum of compound $11 d\left(\mathrm{CDCl}_{3}, 101 \mathrm{MHz}\right)$ 


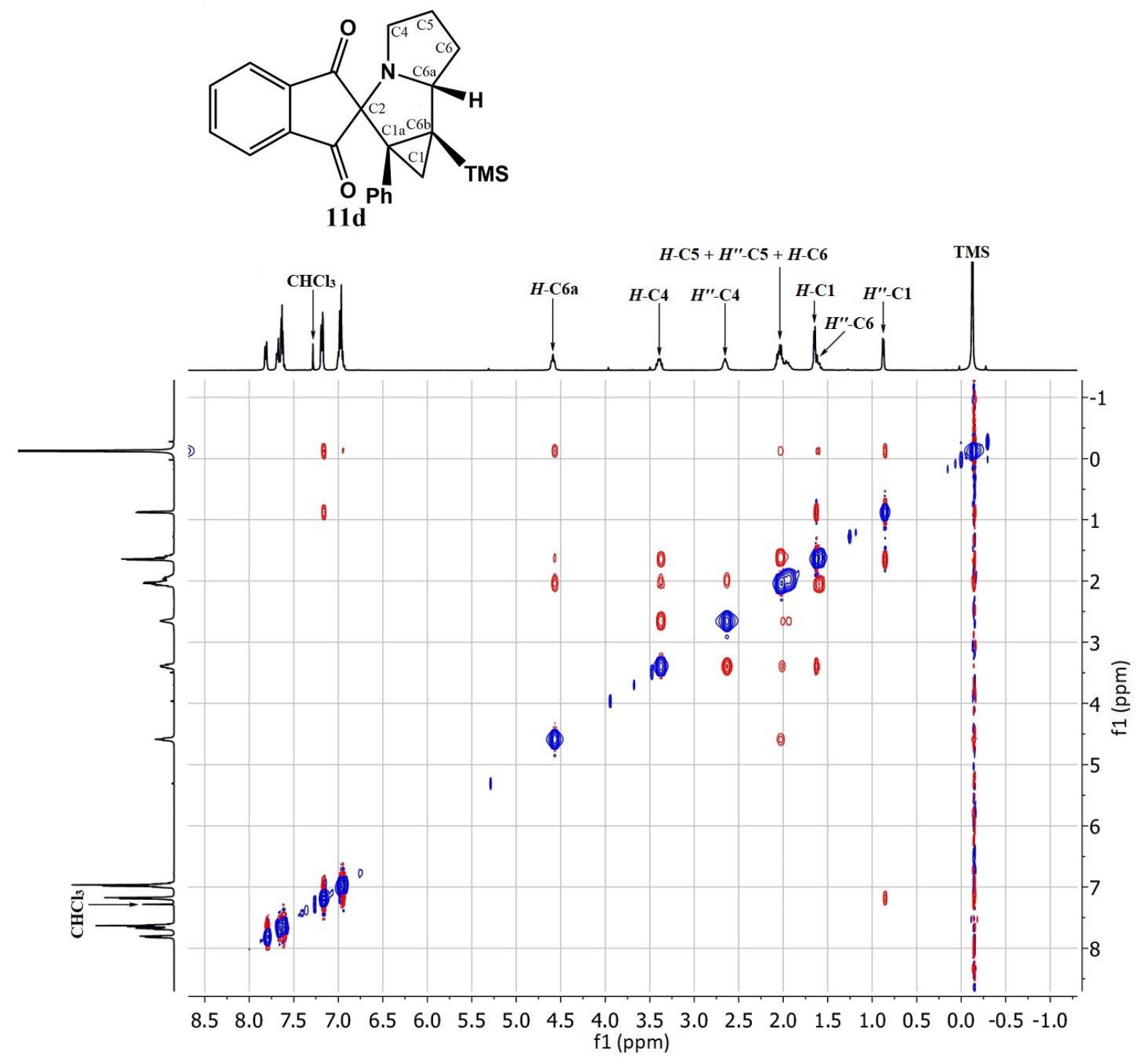

Figure S45. 2D ${ }^{1} \mathrm{H}-{ }^{1} \mathrm{H}$ NOESY spectrum of compound $11 d\left(\mathrm{CDCl}_{3}, 400 \mathrm{MHz}\right)$ 
${ }^{1} \mathrm{H}$ NMR (400 MHz, $\left.\mathrm{CDCl}_{3}\right)$
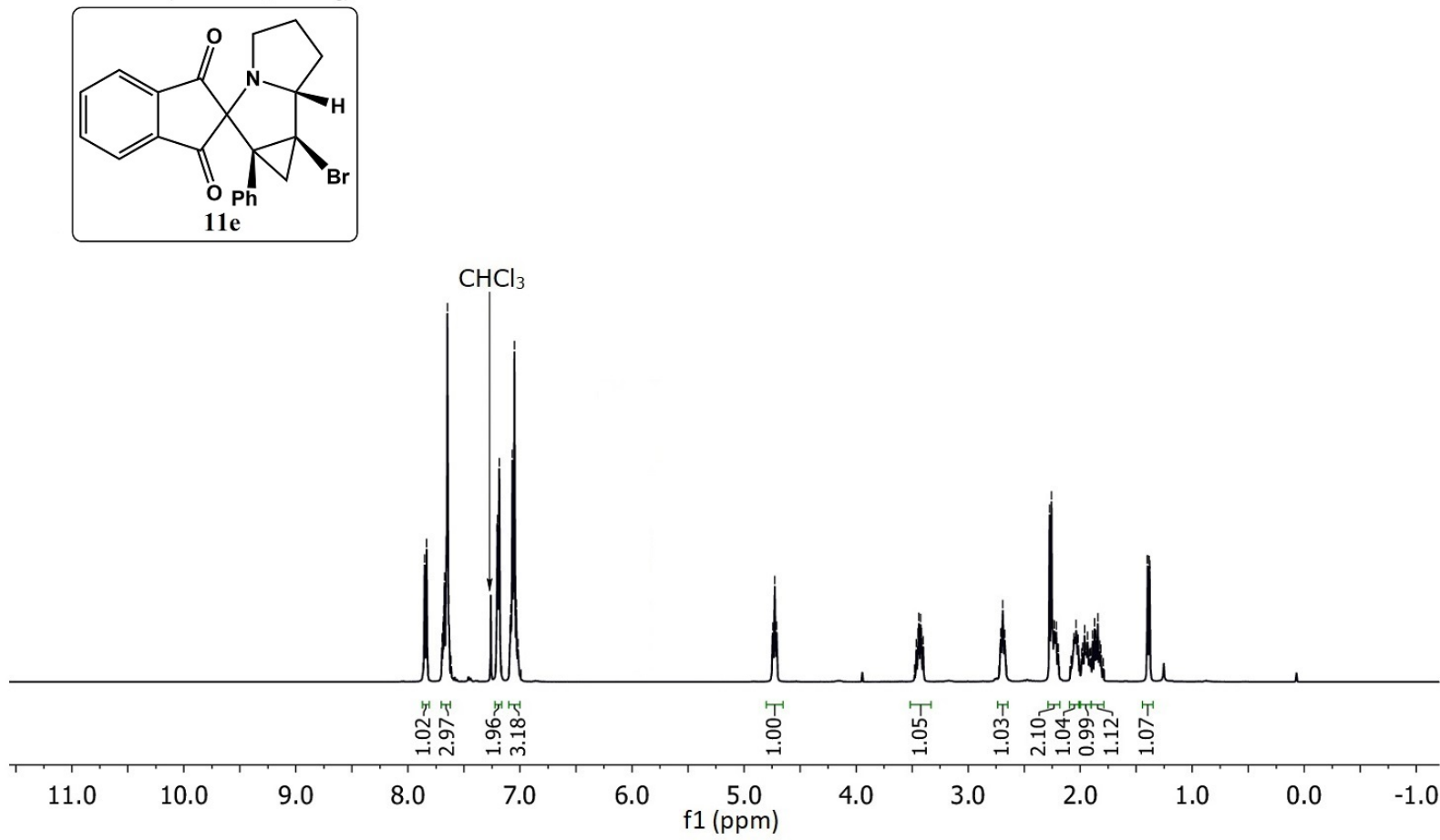

Figure S46. ${ }^{1} \mathrm{H}$ NMR spectrum of compound $11 e\left(\mathrm{CDCl}_{3}, 400 \mathrm{MHz}\right)$

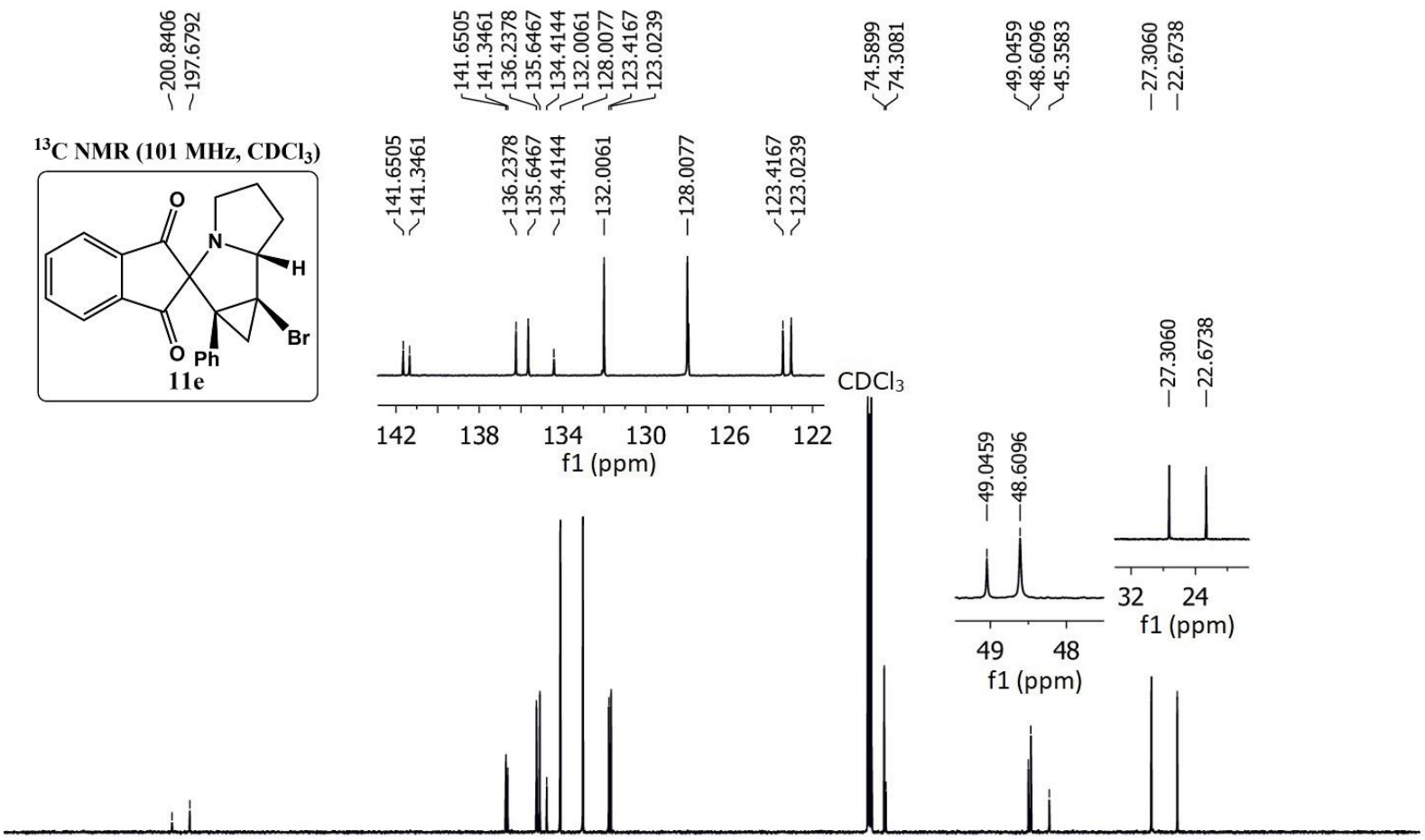

$\begin{array}{llllllllllllllllllllllll}220 & 210 & 200 & 190 & 180 & 170 & 160 & 150 & 140 & 130 & 120 & \begin{array}{l}110 \\ \mathrm{f} 1(\mathrm{ppm})\end{array}\end{array}$

Figure S47. ${ }^{13} \mathrm{C}$ NMR spectrum of compound $11 e\left(\mathrm{CDCl}_{3}, 101 \mathrm{MHz}\right)$ 


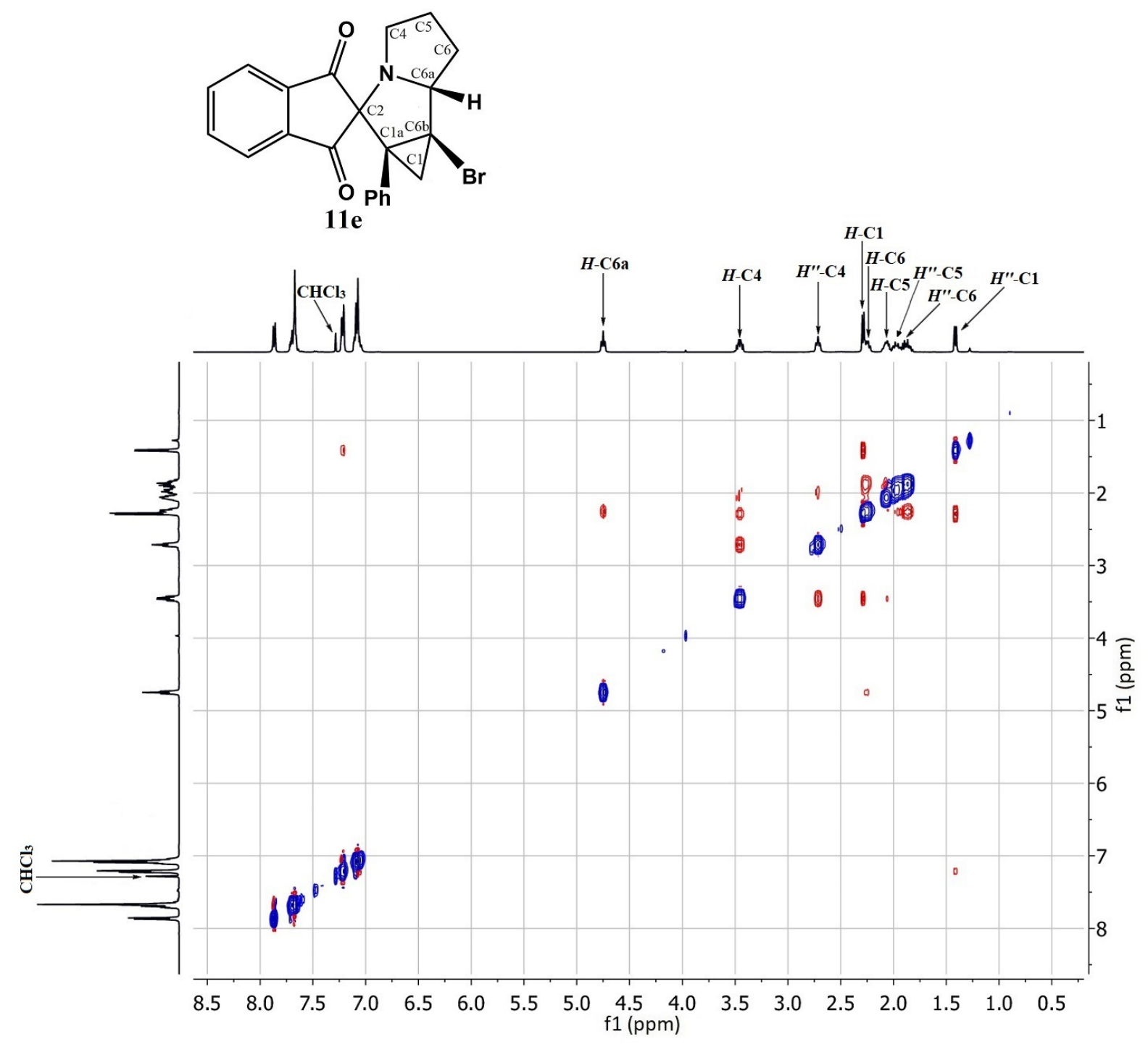

Figure S48. 2D ${ }^{1} \mathrm{H}-{ }^{1} \mathrm{H}$ NOESY spectrum of compound $11 e\left(\mathrm{CDCl}_{3}, 400 \mathrm{MHz}\right)$ 


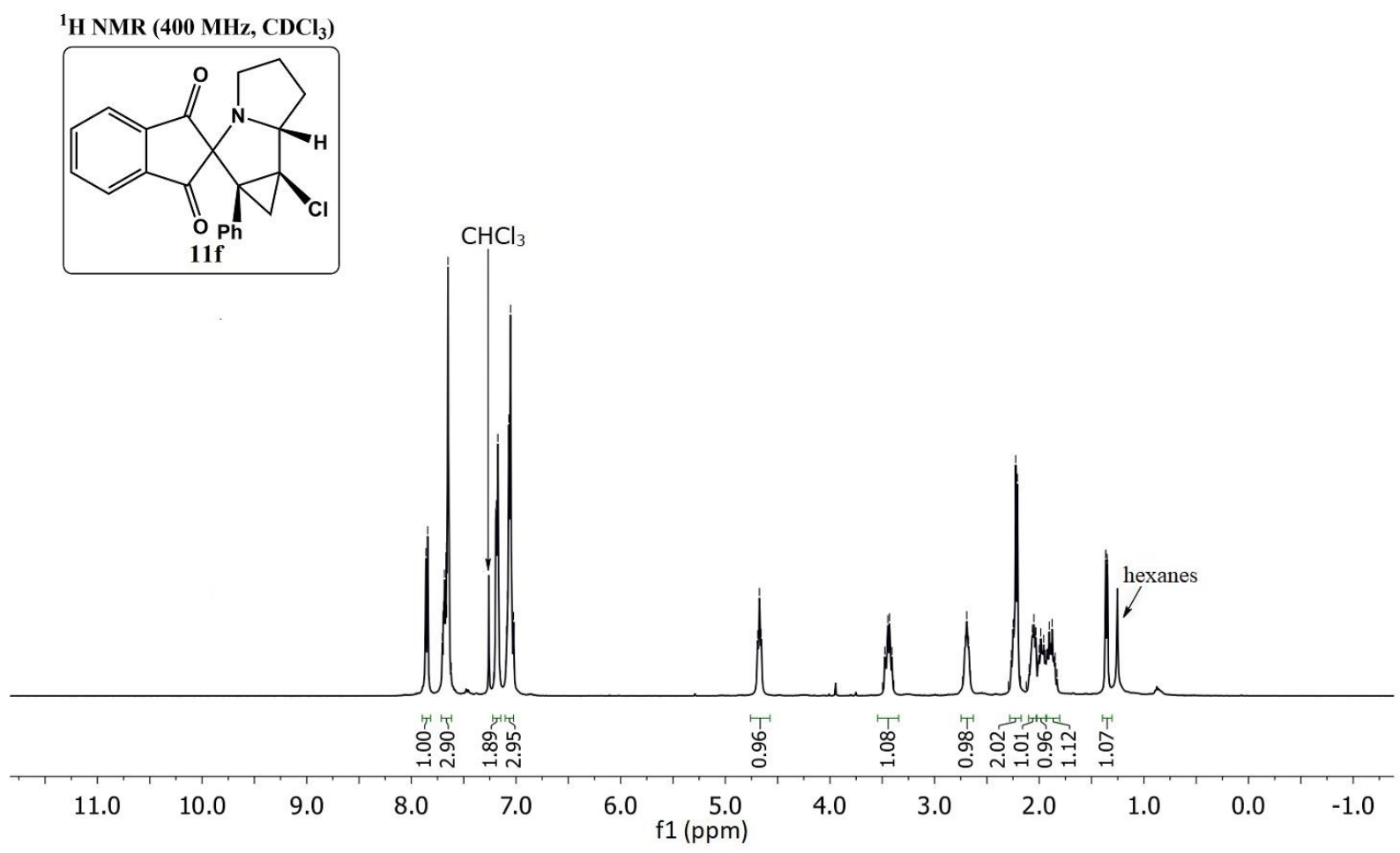

Figure S49. ${ }^{1} \mathrm{H}$ NMR spectrum of compound $11 f\left(\mathrm{CDCl}_{3}, 400 \mathrm{MHz}\right)$
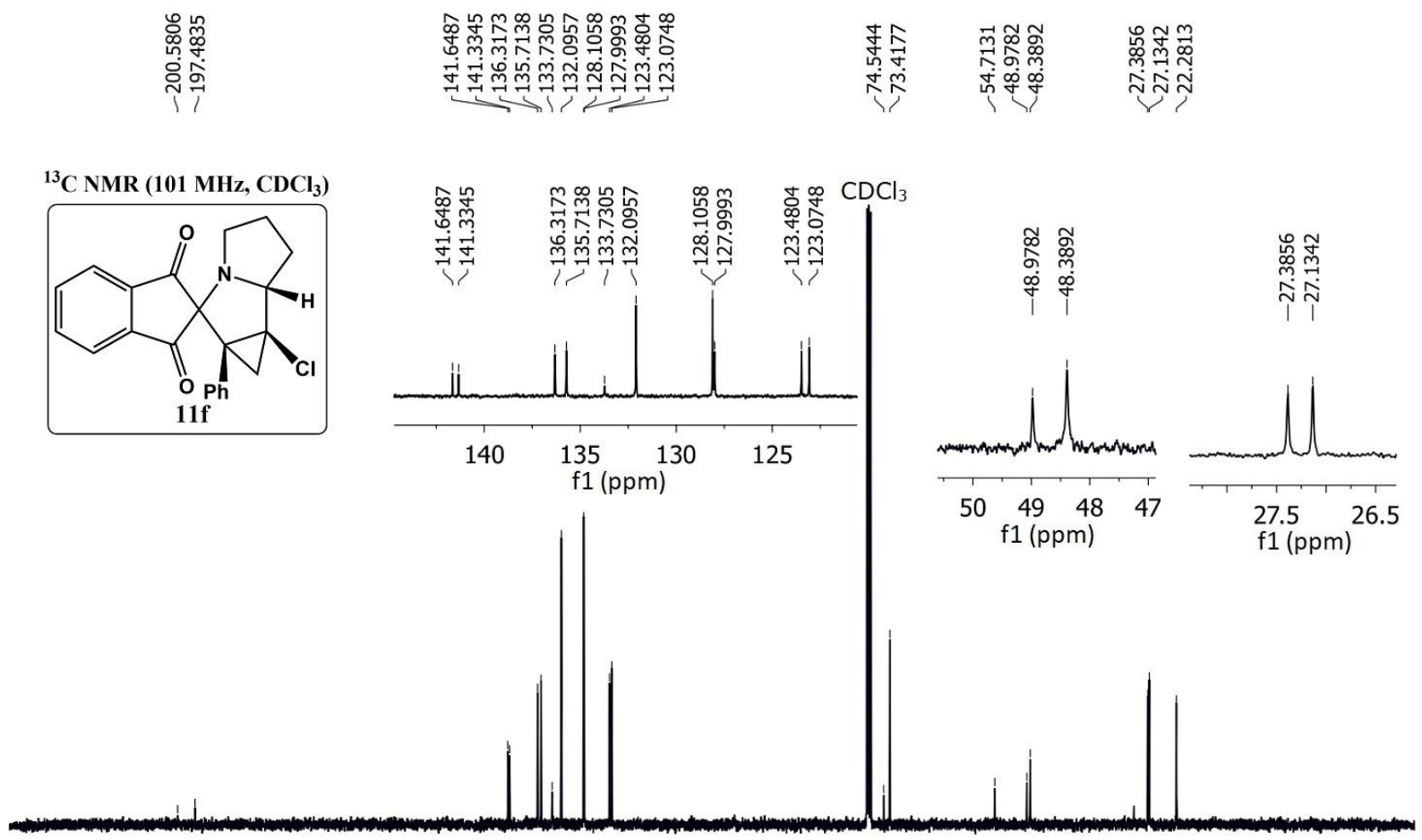

$\begin{array}{llllllllllllllllllllllll}220 & 210 & 200 & 190 & 180 & 170 & 160 & 150 & 140 & 130 & 120 & 110 \quad 100 & 90 & 80 & 70 & 60 & 50 & 40 & 30 & 20 & 10 & 0 & -10\end{array}$

Figure S50. ${ }^{13} \mathrm{C}$ NMR spectrum of compound $11 f\left(\mathrm{CDCl}_{3}, 101 \mathrm{MHz}\right)$ 


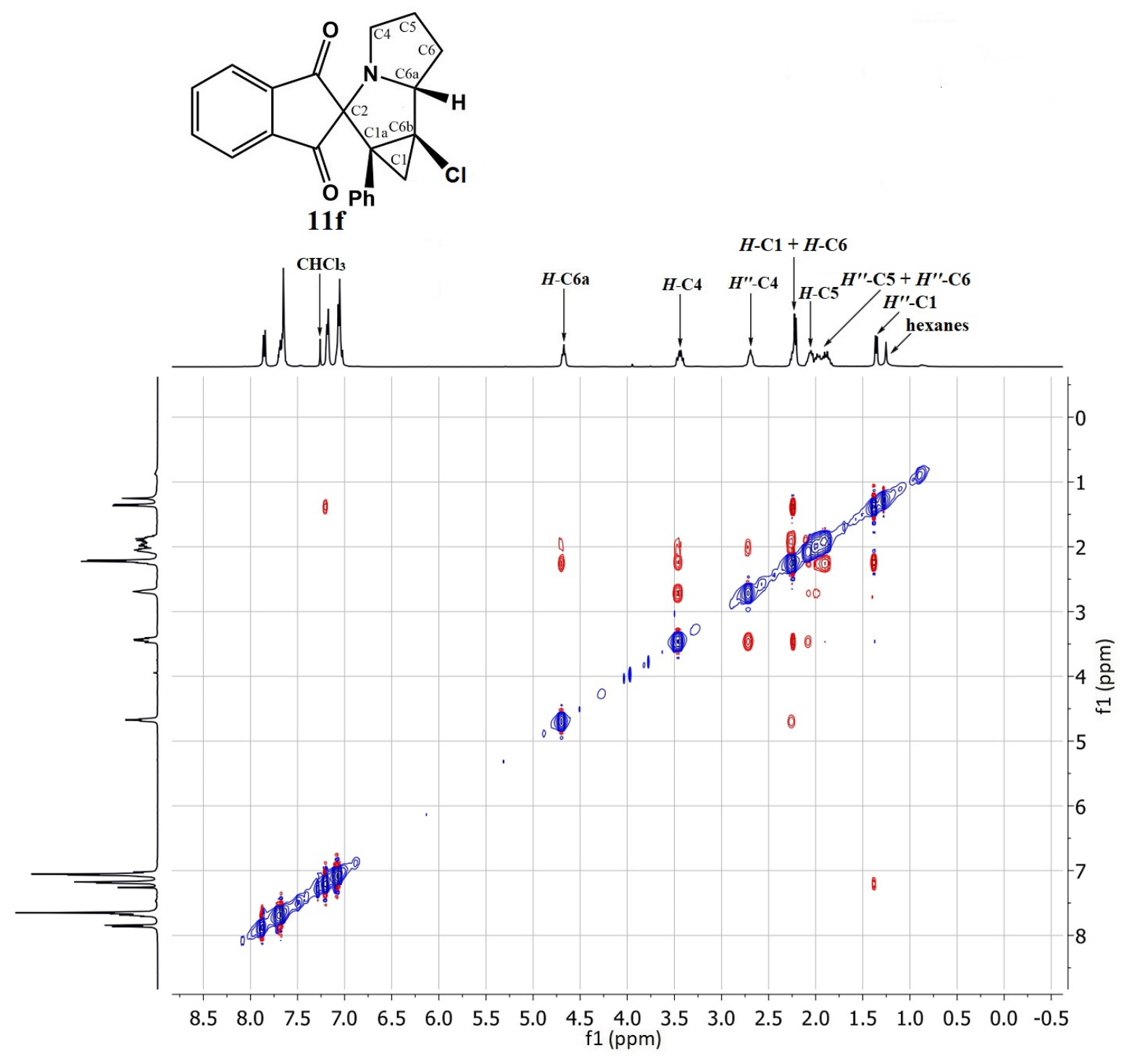

Figure S51. 2D ${ }^{1} \mathrm{H}-{ }^{1} \mathrm{H}$ NOESY spectrum of compound $11 f\left(\mathrm{CDCl}_{3}, 400 \mathrm{MHz}\right)$ 

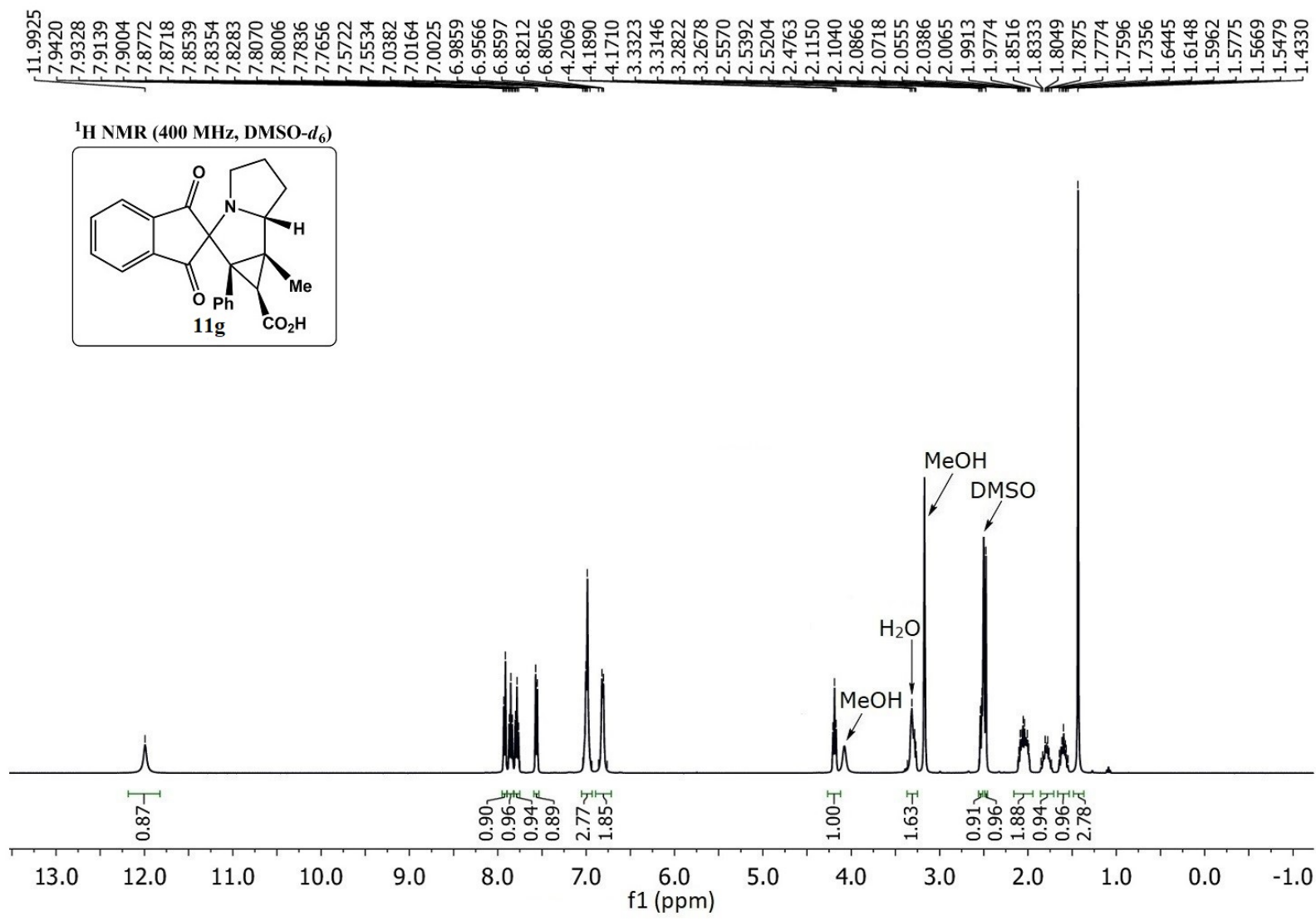

Figure S52. ${ }^{1} \mathrm{H}$ NMR spectrum of compound 11g (DMSO- $\left.d_{6}, 400 \mathrm{MHz}\right)$

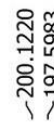

${ }^{13} \mathrm{C}$ NMR (101 MHz, DMSO- $d_{6}$ )

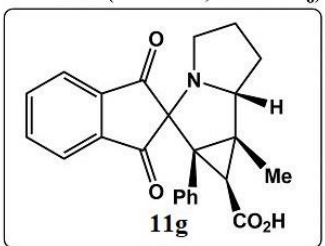

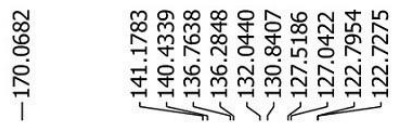

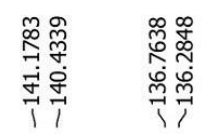

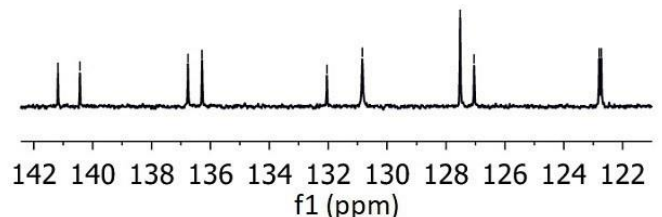

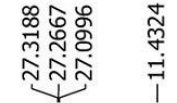

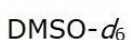

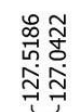

苋鉴

ลิำ

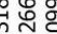

กิก

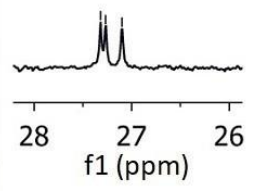

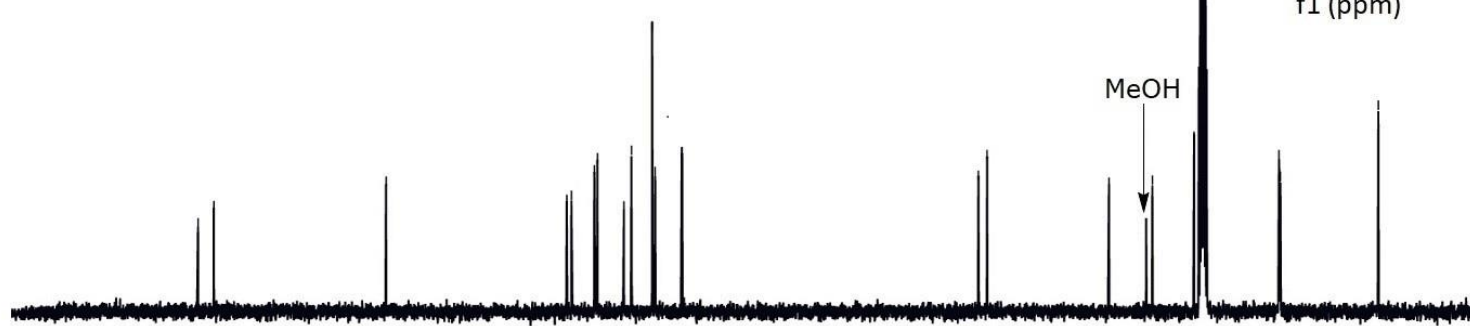

$\begin{array}{llllllllllllllllllllllll}220 & 210 & 200 & 190 & 180 & 170 & 160 & 150 & 140 & 130 & 120 & 110 \quad 100 & 90 & 80 & 70 & 60 & 50 & 40 & 30 & 20 & 10 & 0 & -10\end{array}$

Figure S53. ${ }^{13} \mathrm{C}$ NMR spectrum of compound $11 \mathrm{~g}\left(\mathrm{DMSO}-d_{6}, 101 \mathrm{MHz}\right)$ 


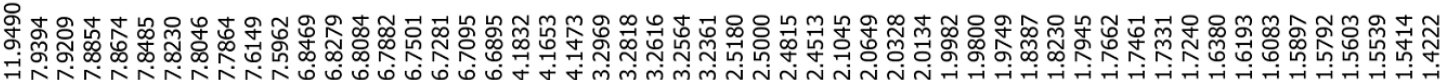

${ }^{1}$ H NMR (400 MHz, DMSO- $\left.d_{6}\right)$
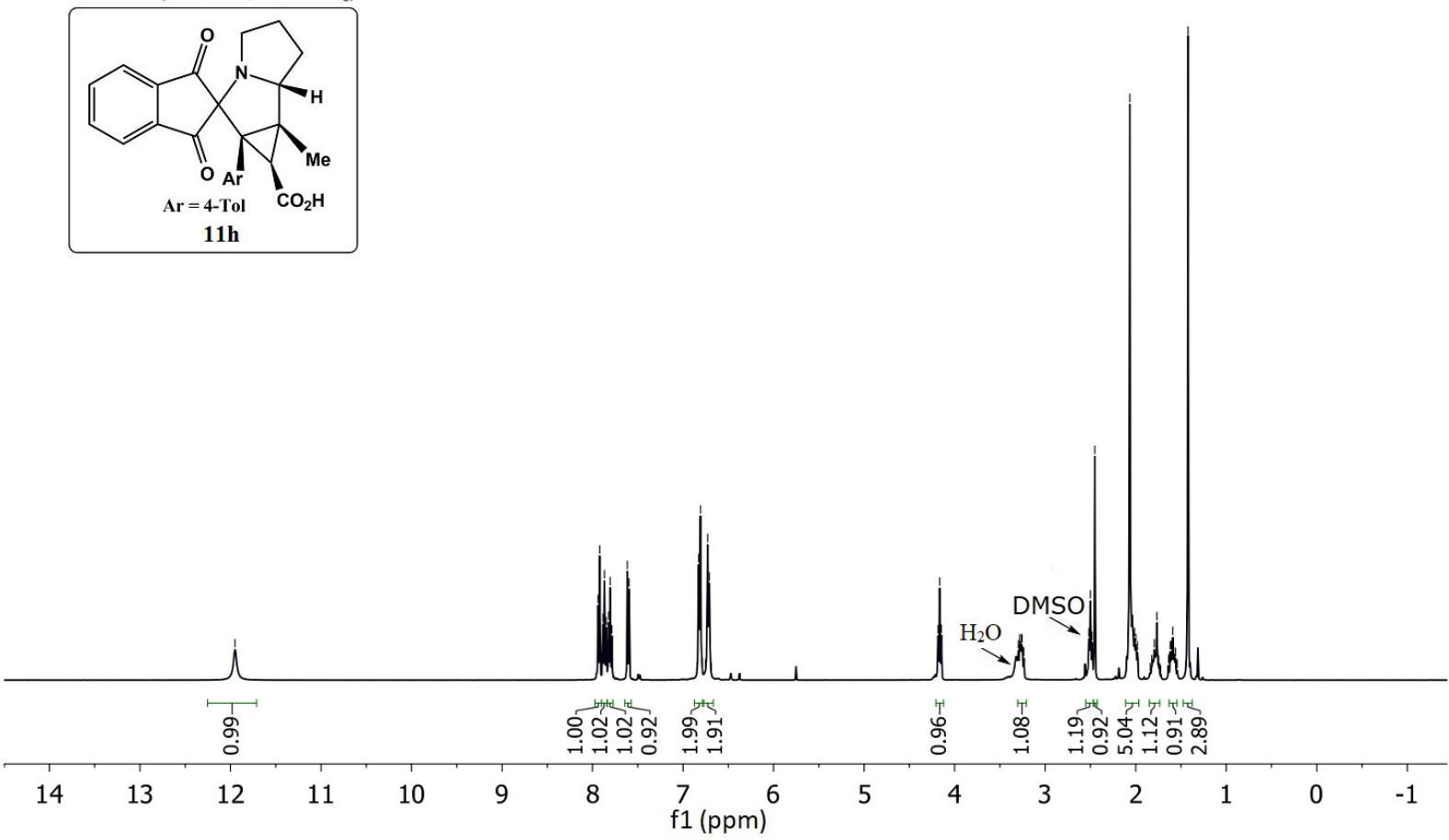

Figure S54. ${ }^{1} \mathrm{H}$ NMR spectrum of compound $11 \mathrm{~h}\left(\mathrm{DMSO}-d_{6}, 400 \mathrm{MHz}\right)$
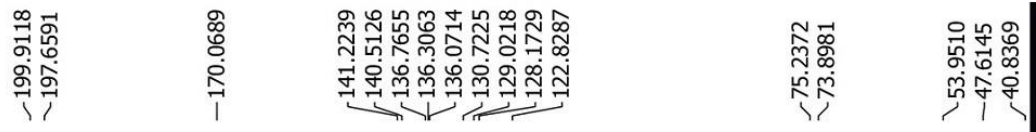

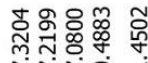

ลิ่งิัก

${ }^{13}$ C NMR (101 MHz, DMSO- $d_{6}$ )

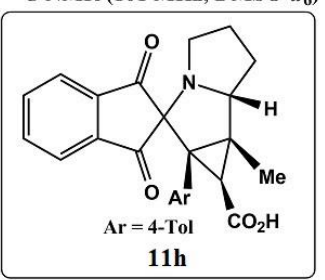

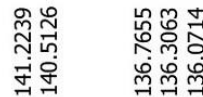

노ำ

จิ่

DMSO- $d_{6}$
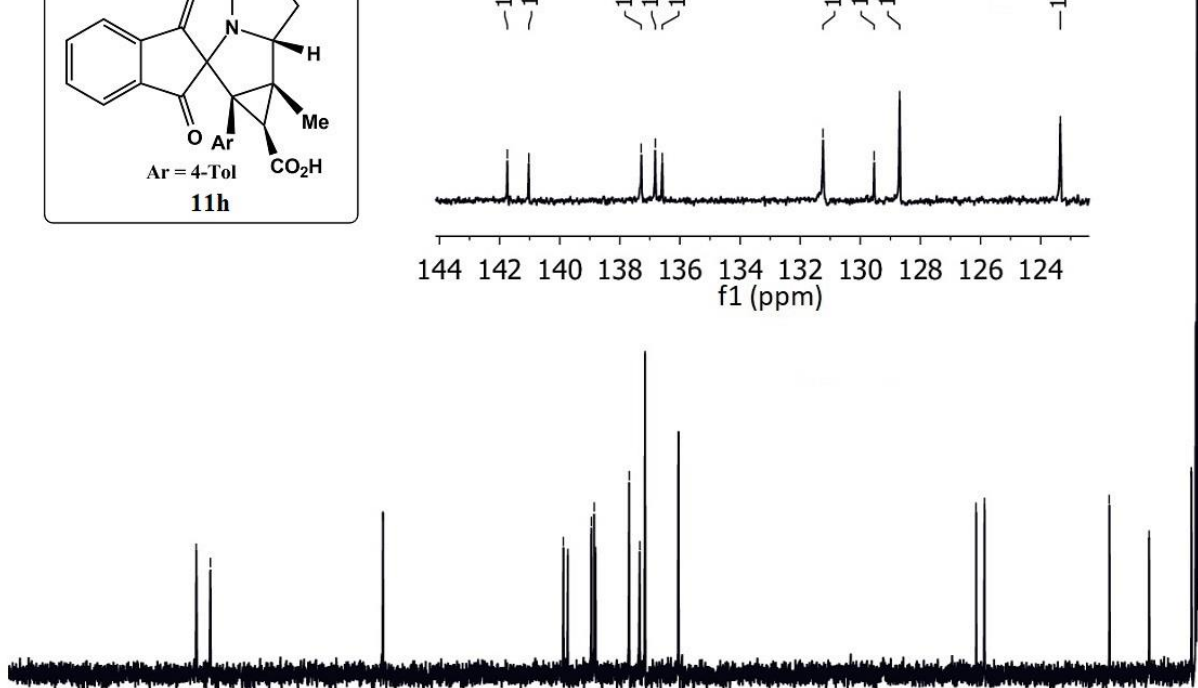

.

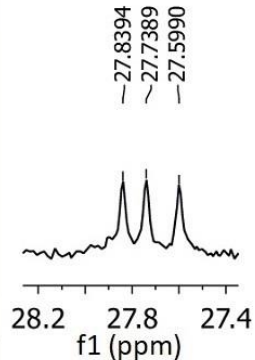

$\begin{array}{llllllllllllllllllllllll}220 & 210 & 200 & 190 & 180 & 170 & 160 & 150 & 140 & 130 & 120 & 110 \quad 100 & 90 & 80 & 70 & 60 & 50 & 40 & 30 & 20 & 10 & 0 & -10\end{array}$

Figure S55. ${ }^{13} \mathrm{C}$ NMR spectrum of compound $11 \mathrm{~h}\left(\mathrm{DMSO}-d_{6}, 101 \mathrm{MHz}\right)$ 


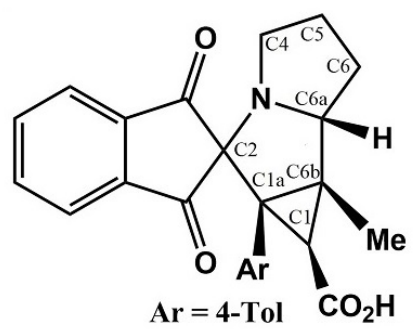

$11 \mathrm{~h}$

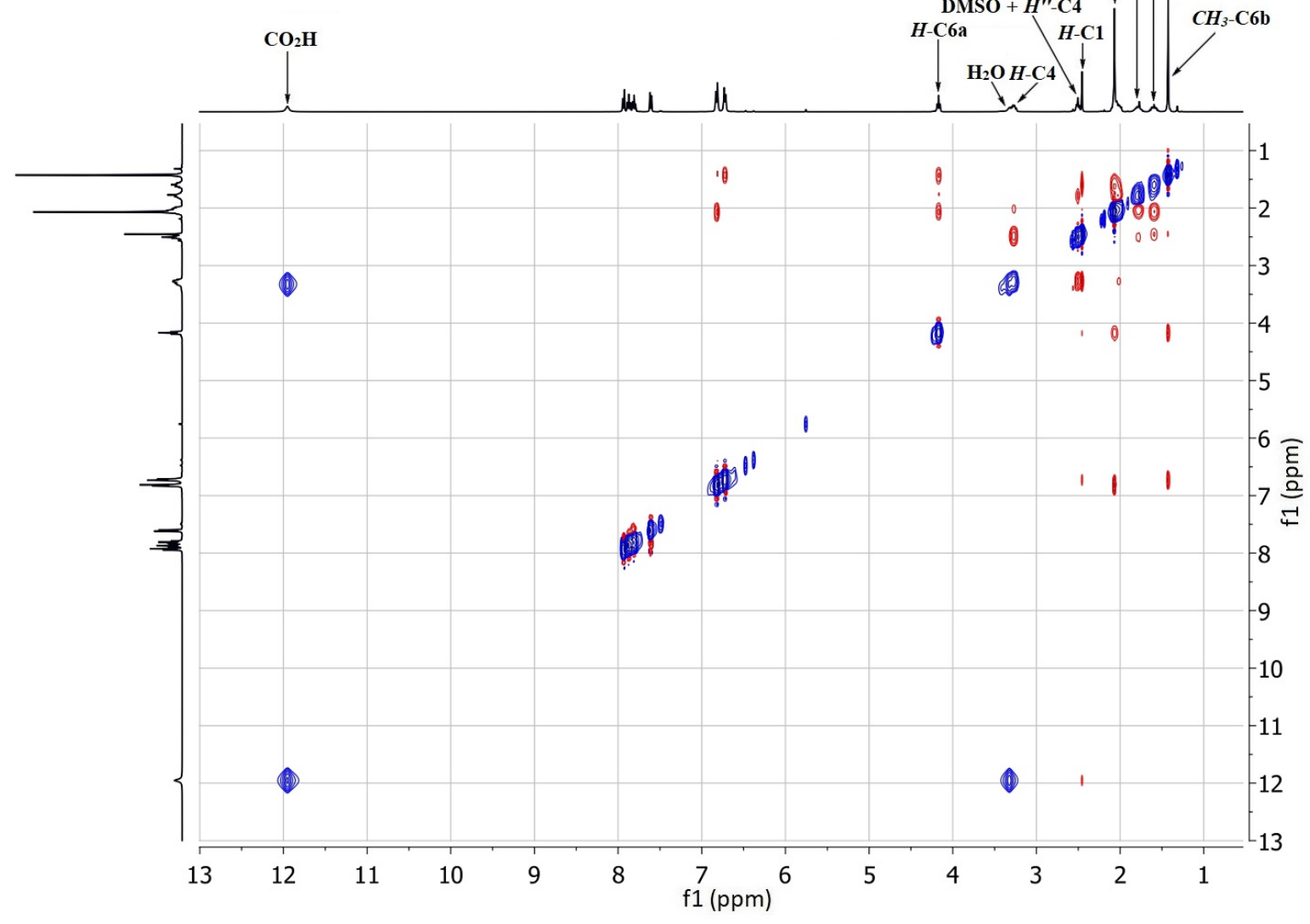

Figure S56. $2 \mathrm{D}^{1} \mathrm{H}-{ }^{1} \mathrm{H}$ NOESY spectrum of compound $\mathbf{1 1 h}\left(\mathrm{CDCl}_{3}, 400 \mathrm{MHz}\right)$ 

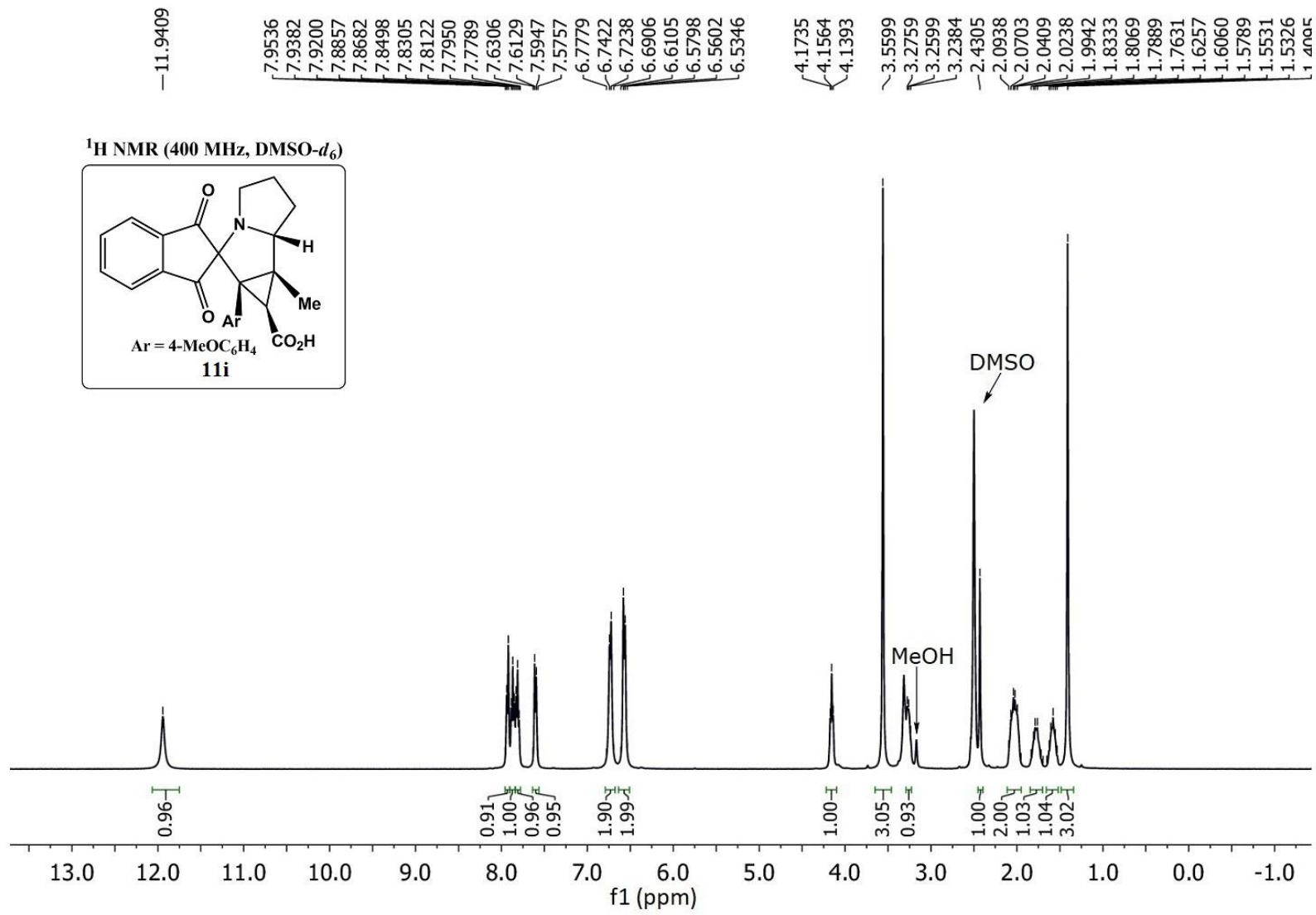

Figure S57. ${ }^{1} \mathrm{H}$ NMR spectrum of compound 11i (DMSO- $\left.d_{6}, 400 \mathrm{MHz}\right)$

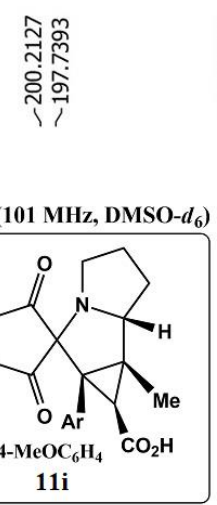

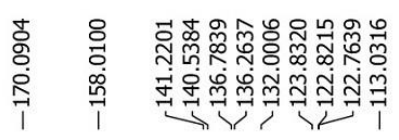
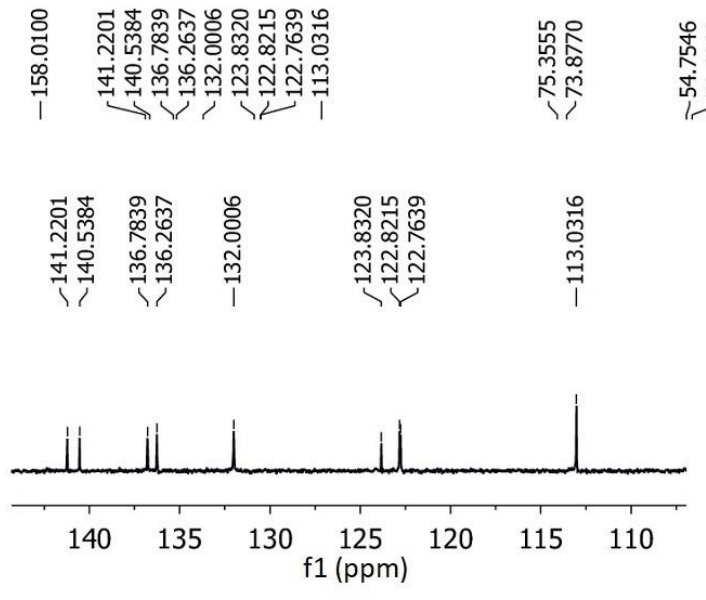

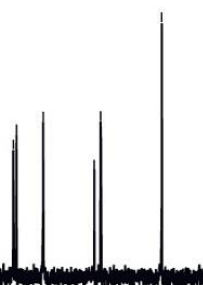

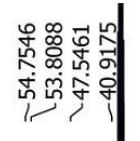

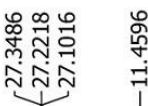

DMSO- $d_{6}$

t

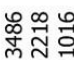

ㅊํㄱ

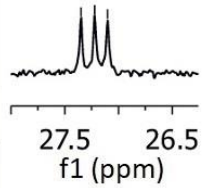

$\begin{array}{lllllllllllllllllllllll}220 & 210 & 200 & 190 & 180 & 170 & 160 & 150 & 140 & 130 & 120 & \begin{array}{c}110 \quad 100 \\ \mathrm{f} 1(\mathrm{ppm})\end{array} & 90 & 80 & 70 & 60 & 50 & 40 & 30 & 20 & 10 & 0 & -10\end{array}$

Figure S58. ${ }^{13} \mathrm{C}$ NMR spectrum of compound 11i $\left(\mathrm{DMSO}-d_{6}, 101 \mathrm{MHz}\right)$ 
${ }^{1} \mathrm{H}$ NMR (400 MHz, $\left.\mathrm{CDCl}_{3}\right)$
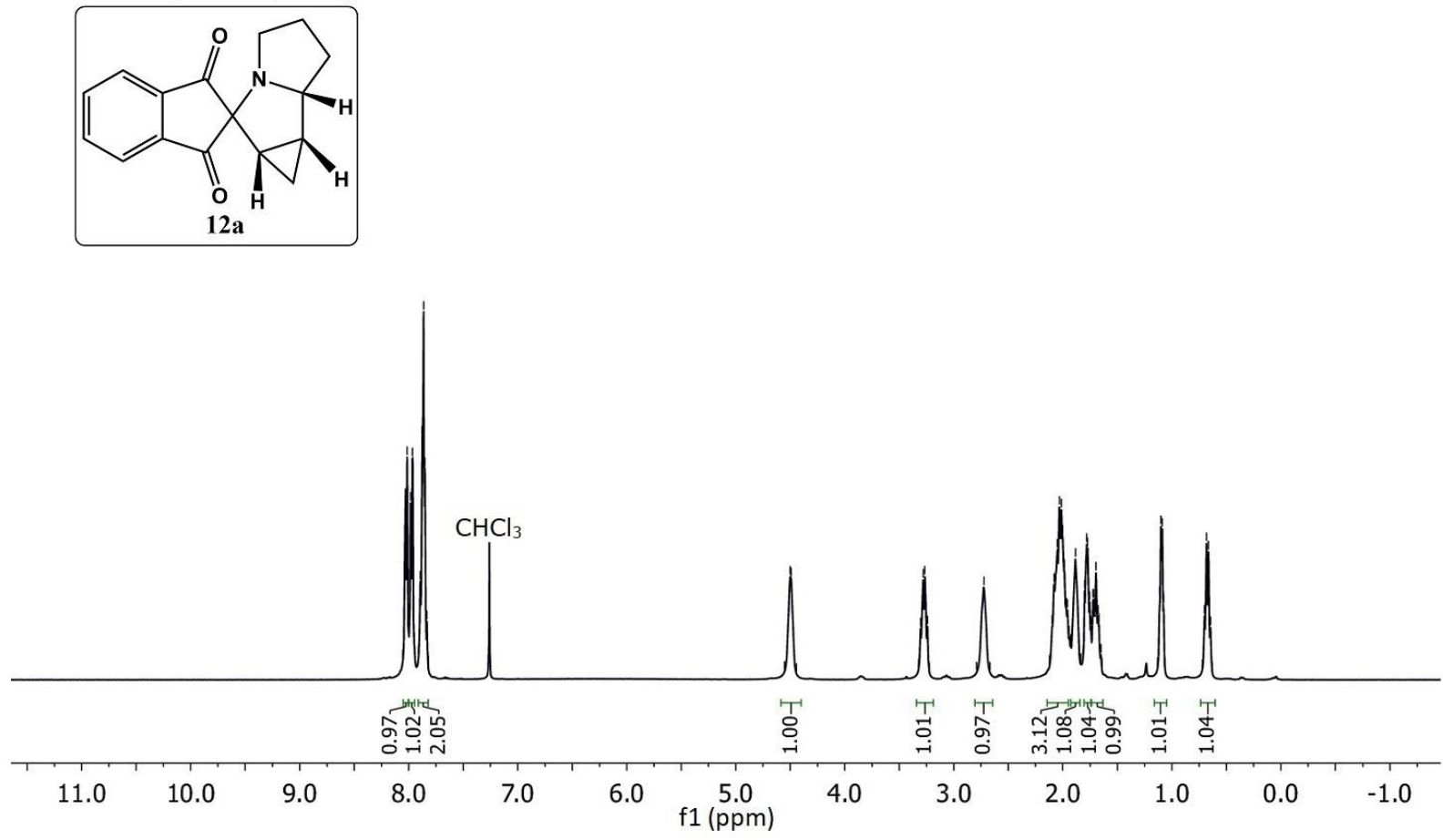

Figure S59. ${ }^{1} \mathrm{H} \mathrm{NMR}$ spectrum of compound 12a $\left(\mathrm{CDCl}_{3}, 400 \mathrm{MHz}\right)$

\section{冬}

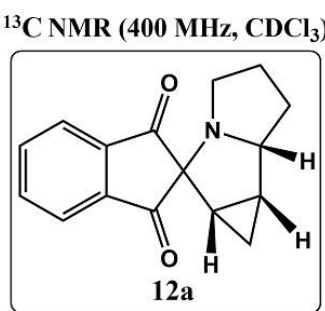

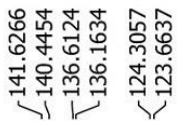
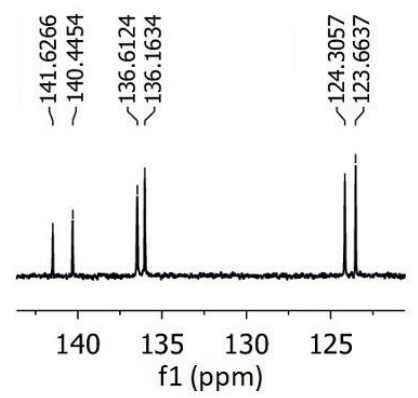

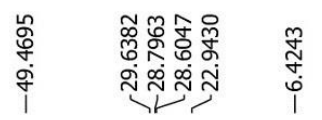

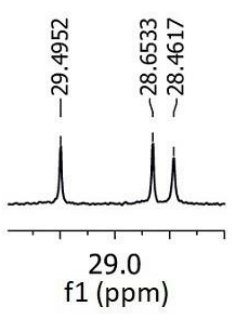

$\begin{array}{llllllllllllllllllllllll}220 & 210 & 200 & 190 & 180 & 170 & 160 & 150 & 140 & 130 & 120 & 110 & 100 & 90 & 80 & 70 & 60 & 50 & 40 & 30 & 20 & 10 & 0 & -10\end{array}$

Figure S60. ${ }^{13} \mathrm{C}$ NMR spectrum of compound 12a $\left(\mathrm{CDCl}_{3}, 101 \mathrm{MHz}\right)$ 


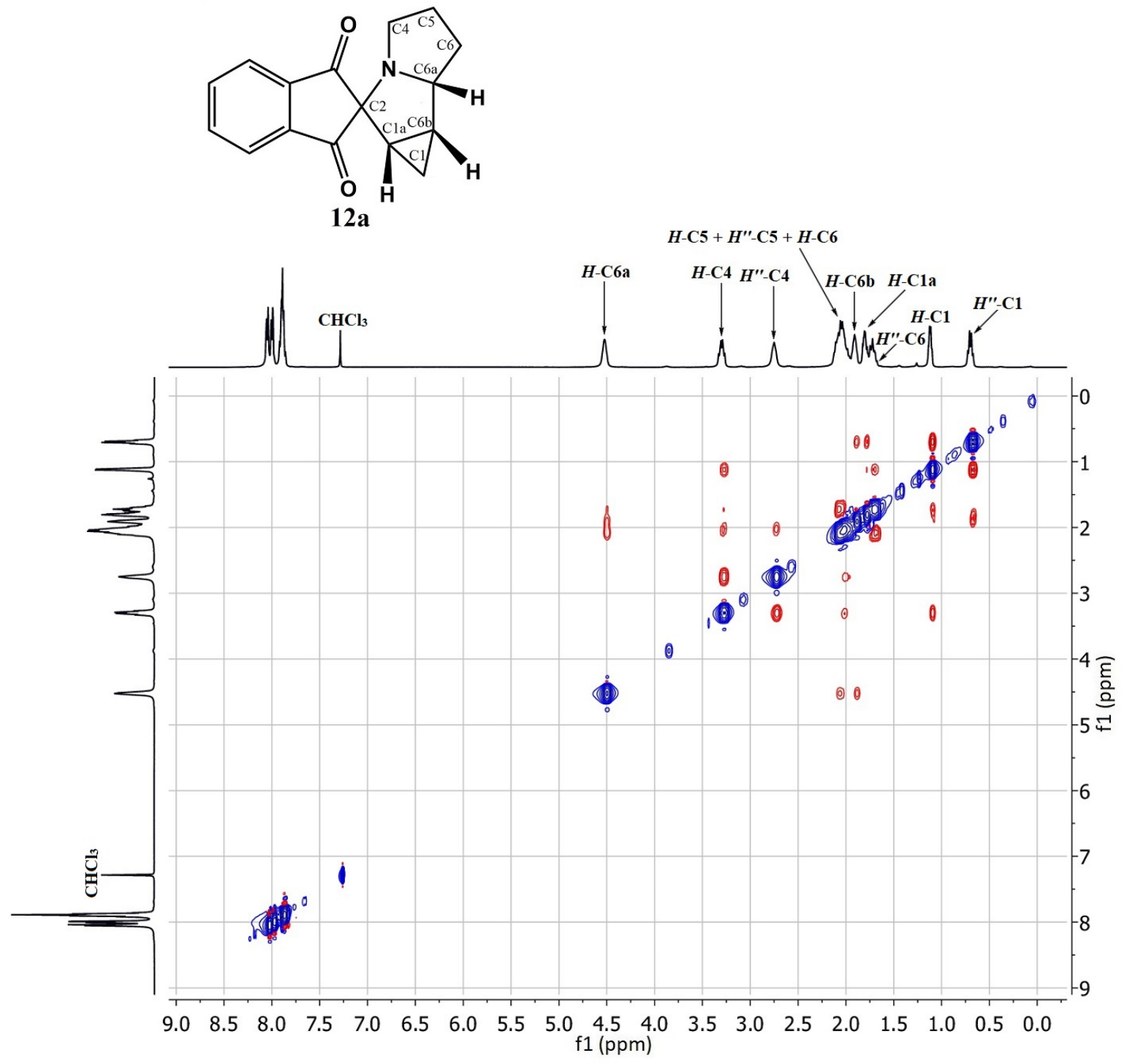

Figure S61. 2D ${ }^{1} \mathrm{H}-{ }^{1} \mathrm{H}$ NOESY spectrum of compound 12a $\left(\mathrm{CDCl}_{3}, 400 \mathrm{MHz}\right)$ 
${ }^{1} \mathrm{H}$ NMR (400 MHz, $\mathrm{CDCl}_{3}$ )
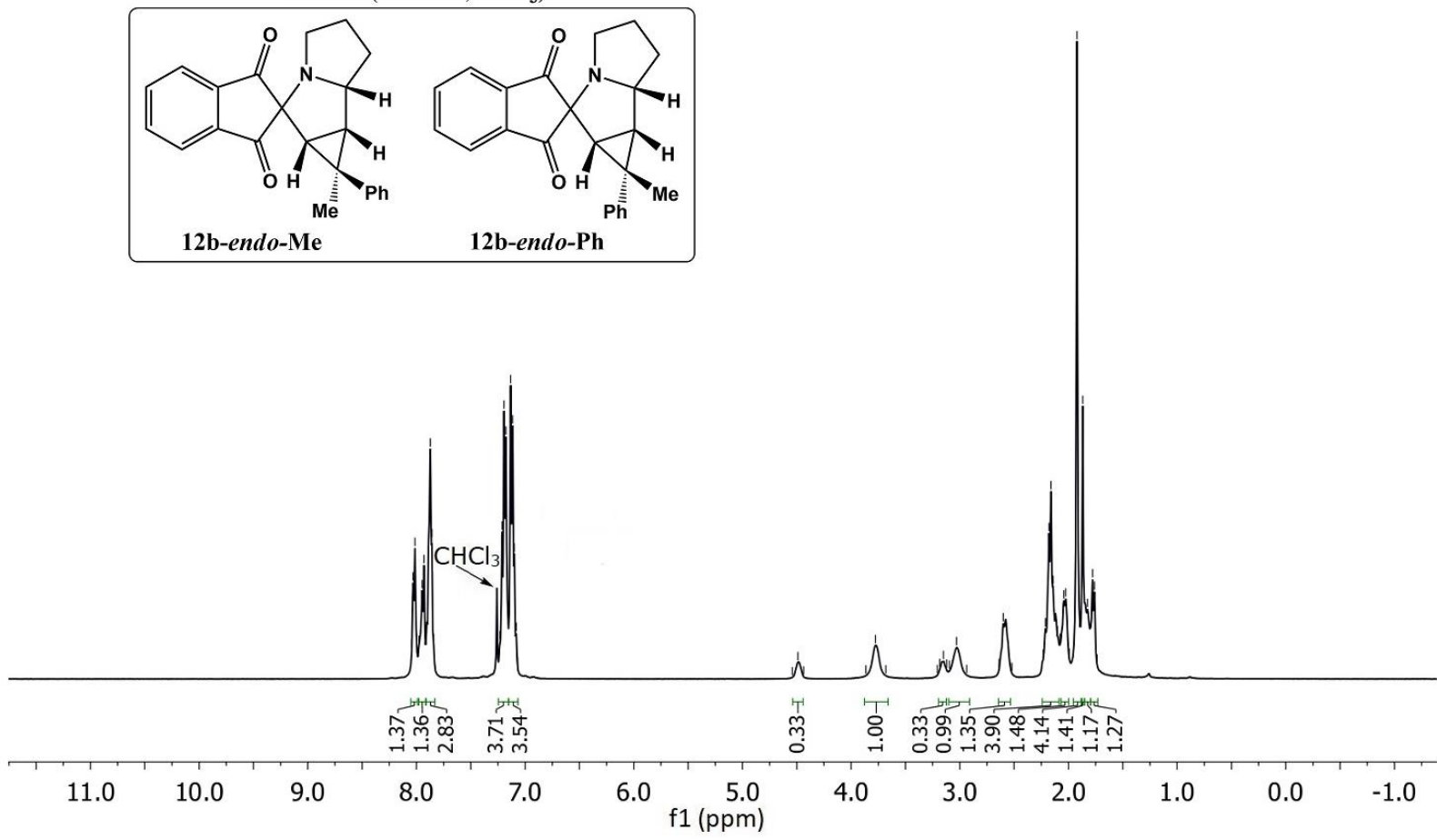

Figure S62. ${ }^{1} \mathrm{H}$ NMR spectrum of diastereomeric mixture 12b-endo-Me and 12b-endo-Ph $\left(\mathrm{CDCl}_{3}, 400 \mathrm{MHz}\right)$

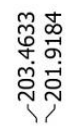

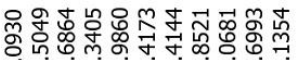

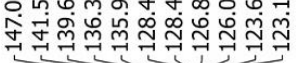

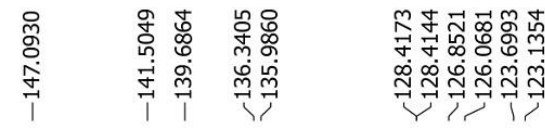

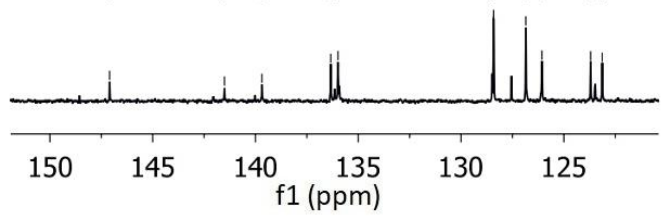

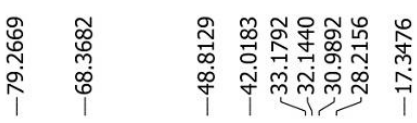

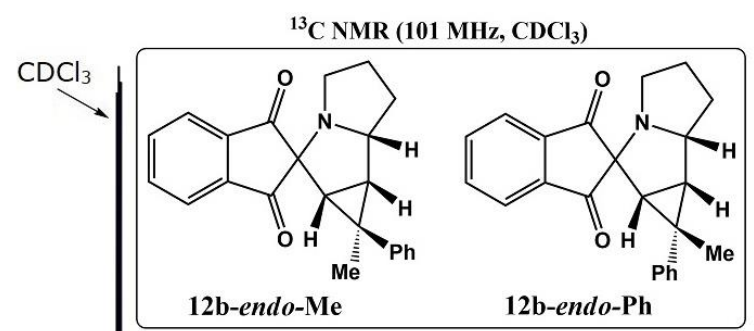

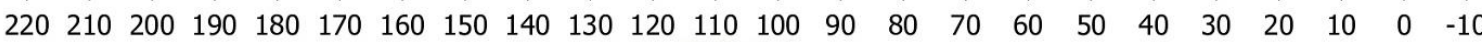
f1 (ppm)

Figure S63. ${ }^{13} \mathrm{C}$ NMR spectrum of diastereomeric mixture 12b-endo-Me and 12b-endo-Ph $\left(\mathrm{CDCl}_{3}, 101 \mathrm{MHz}\right)$ 


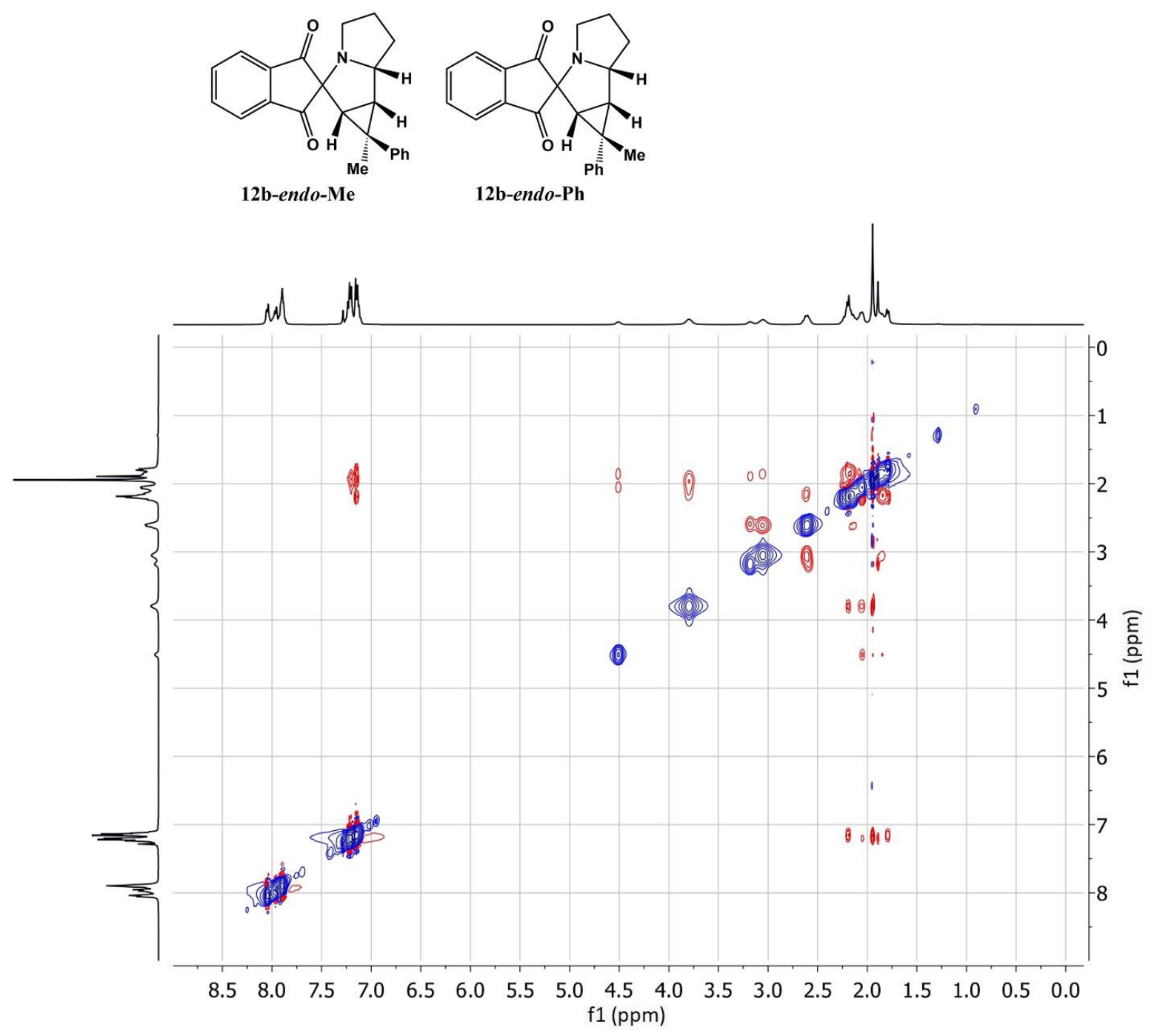

Figure S64. 2D ${ }^{1} \mathrm{H}-{ }^{1} \mathrm{H}$ NOESY spectrum of diastereomeric mixture 12b-endo-Me and 12bendo-Ph $\left(\mathrm{CDCl}_{3}, 400 \mathrm{MHz}\right)$ 


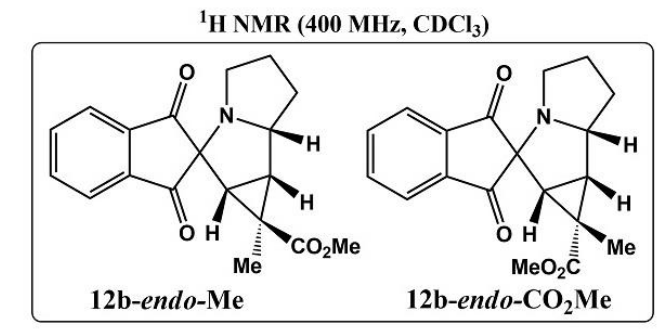

${ }^{1} \mathrm{H}$ NMR (400 MHz, $\left.\mathrm{CDCl}_{3}\right)$
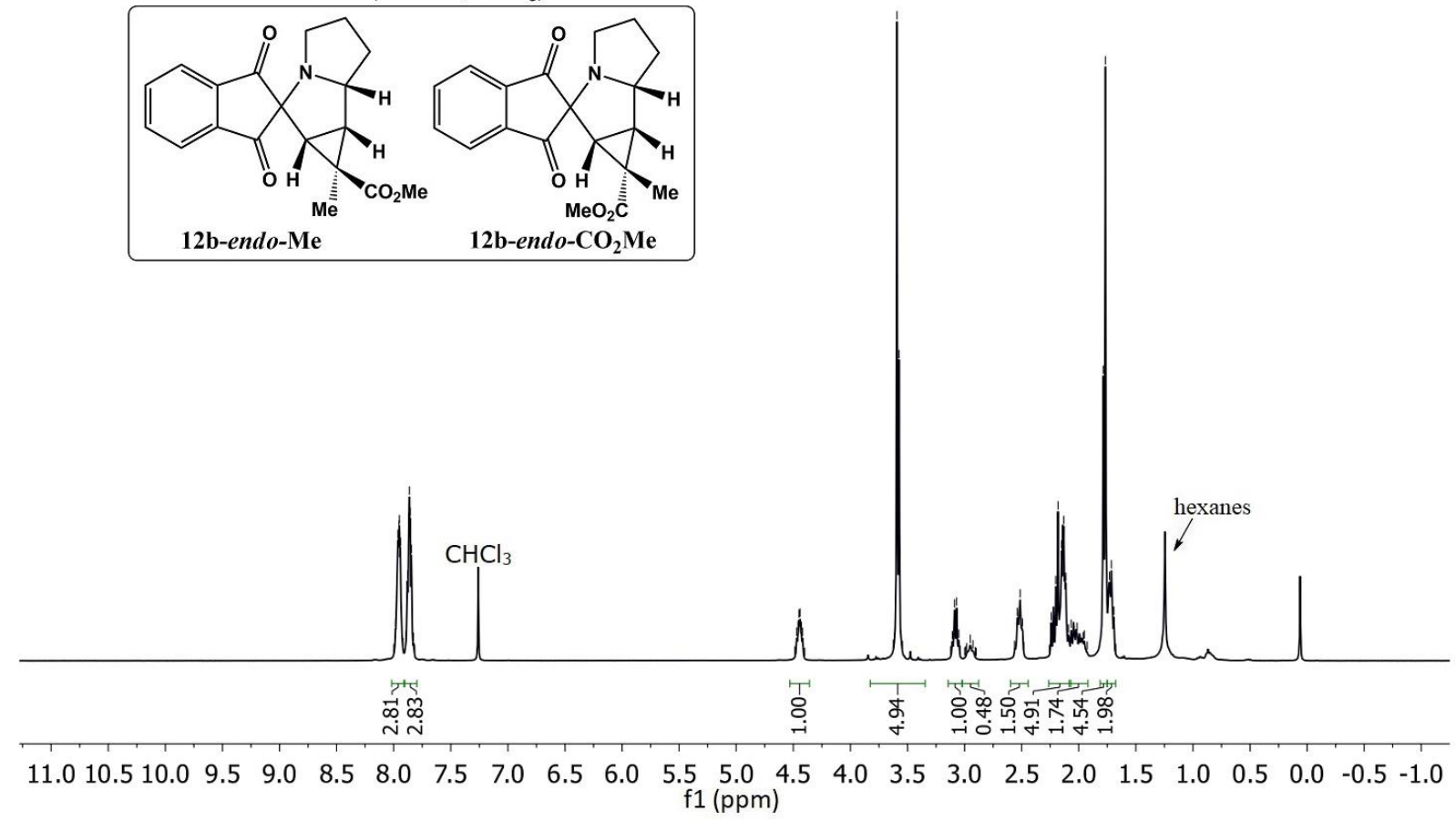

Figure S65. ${ }^{1} \mathrm{H}$ NMR spectrum of diastereomeric mixture $12 \mathrm{c}-$ endo-Me and $12 \mathrm{c}-$ endo- $\mathrm{CO}_{2} \mathrm{Me}$ $\left(\mathrm{CDCl}_{3}, 400 \mathrm{MHz}\right)$
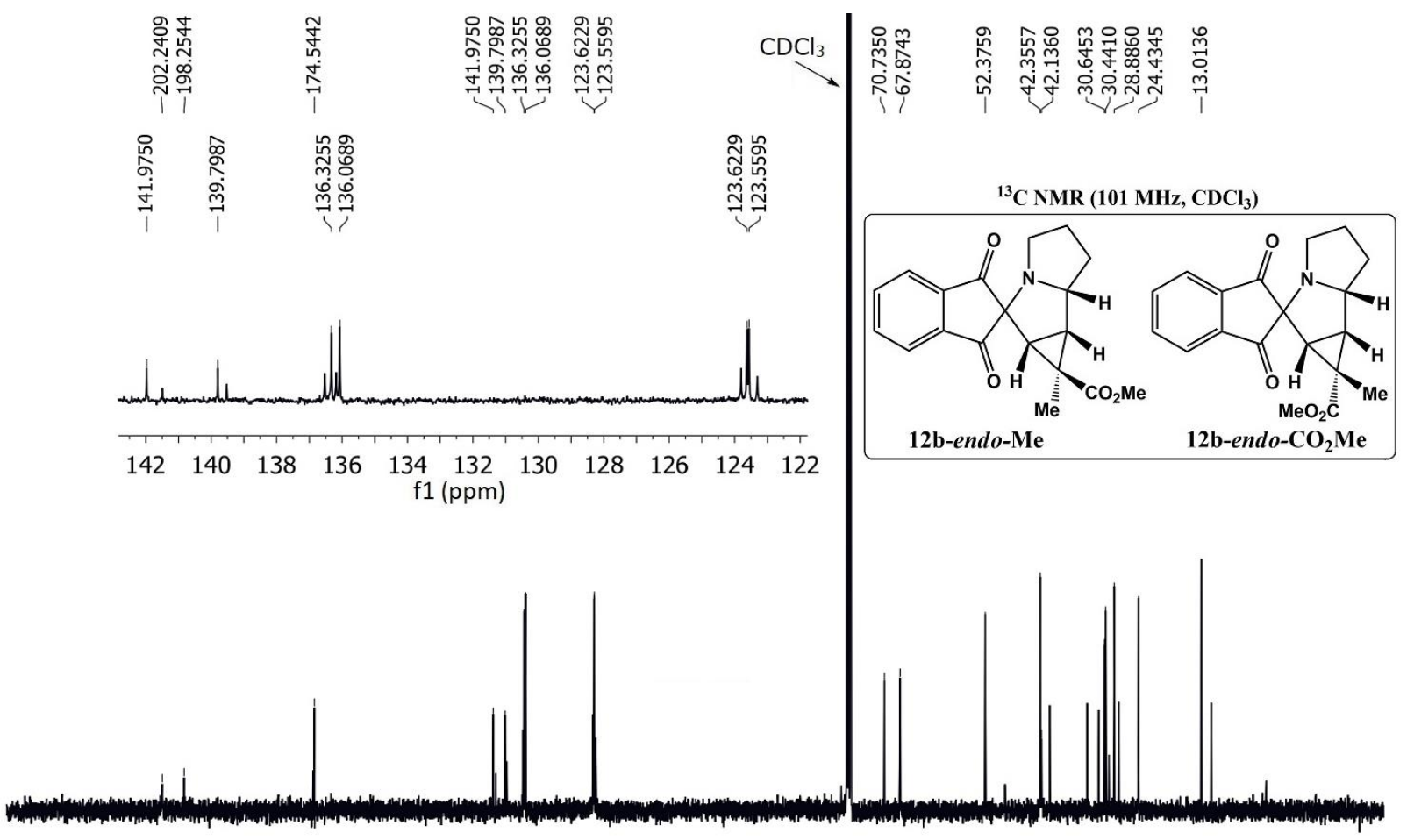

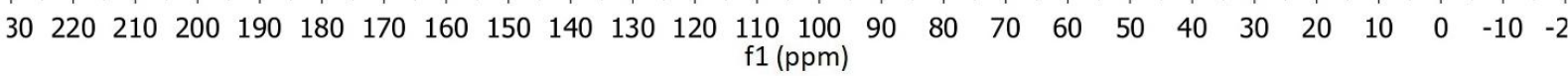

Figure S66. ${ }^{13} \mathrm{C}$ NMR spectrum of diastereomeric mixture 12c-endo-Me and 12c-endo-C $\mathrm{O}_{2} \mathrm{Me}$ $\left(\mathrm{CDCl}_{3}, 101 \mathrm{MHz}\right)$ 


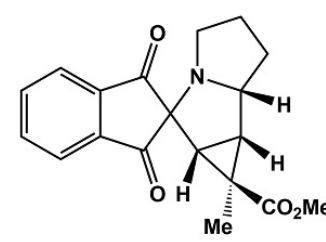

12b-endo-Me

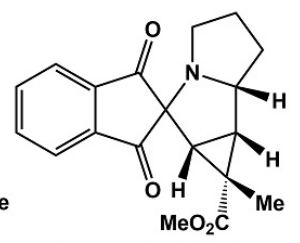

12b-endo- $\mathrm{CO}_{2} \mathrm{Me}$

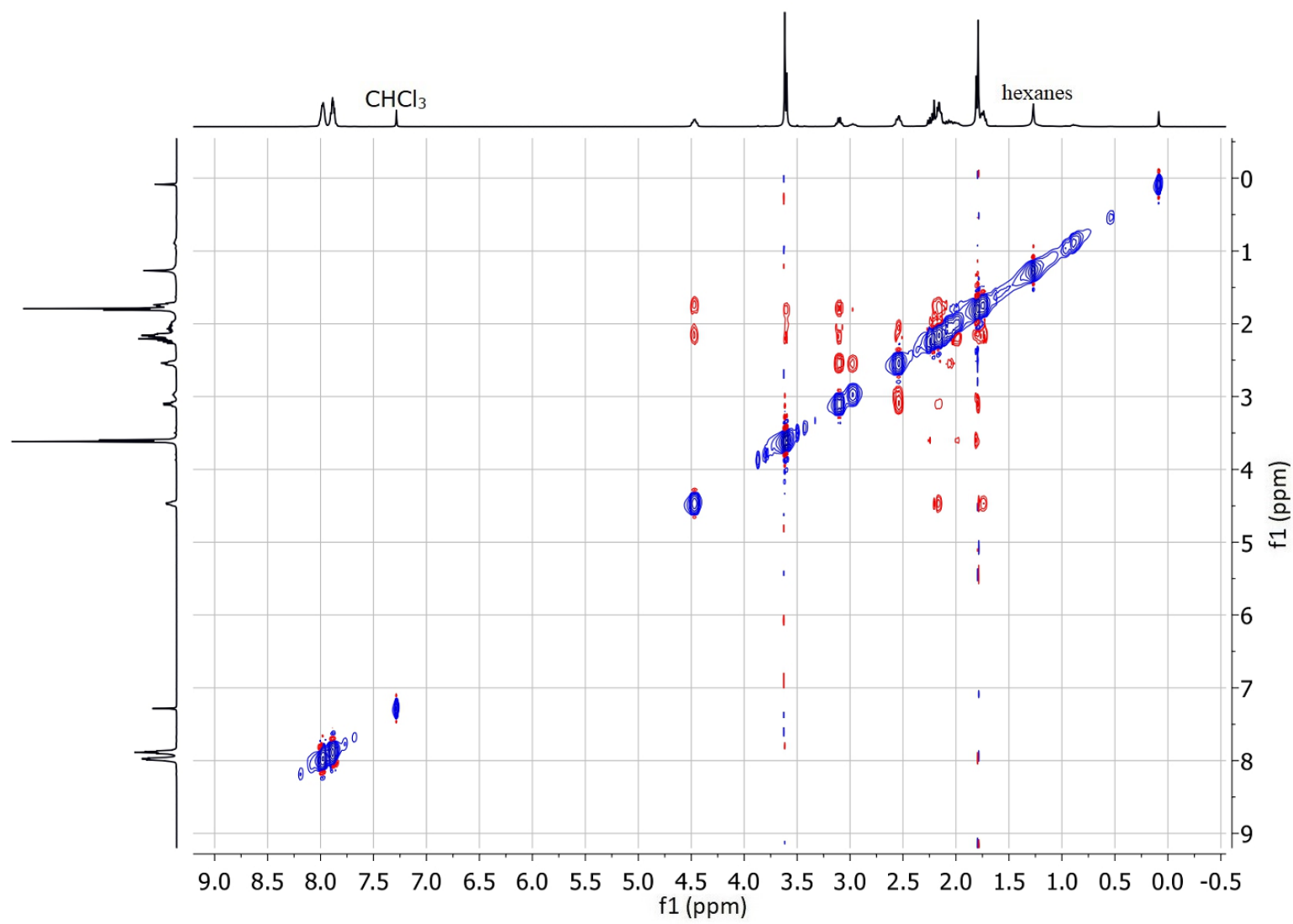

Figure S67. 2D ${ }^{1} \mathrm{H}-{ }^{1} \mathrm{H}$ NOESY spectrum of diastereomeric mixture 12c-endo-Me and 12c-endo$\mathrm{CO}_{2} \mathrm{Me}\left(\mathrm{CDCl}_{3}, 400 \mathrm{MHz}\right)$ 

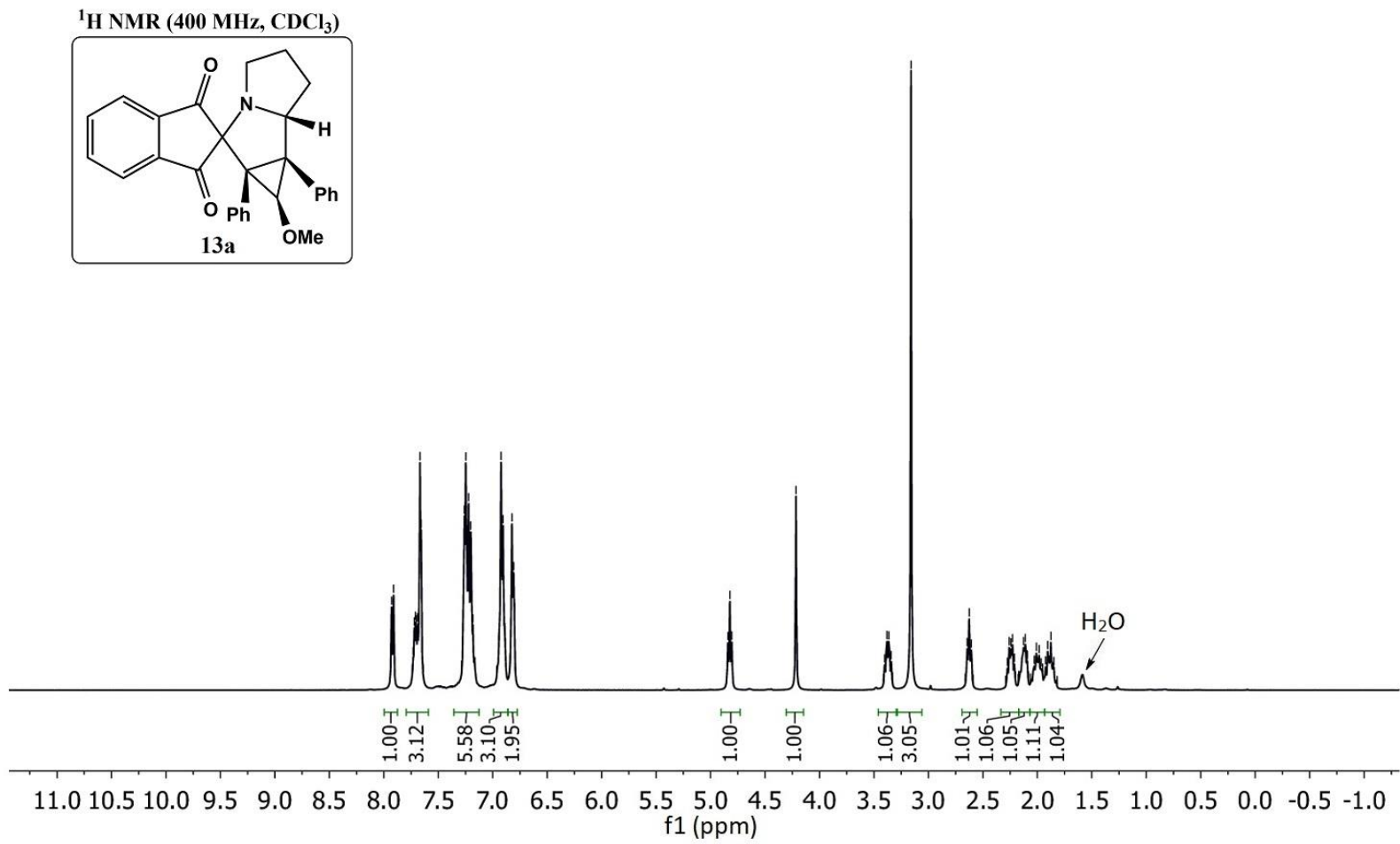

Figure S68. ${ }^{1} \mathrm{H}$ NMR spectrum of compound $13 \mathbf{a}\left(\mathrm{CDCl}_{3}, 400 \mathrm{MHz}\right)$
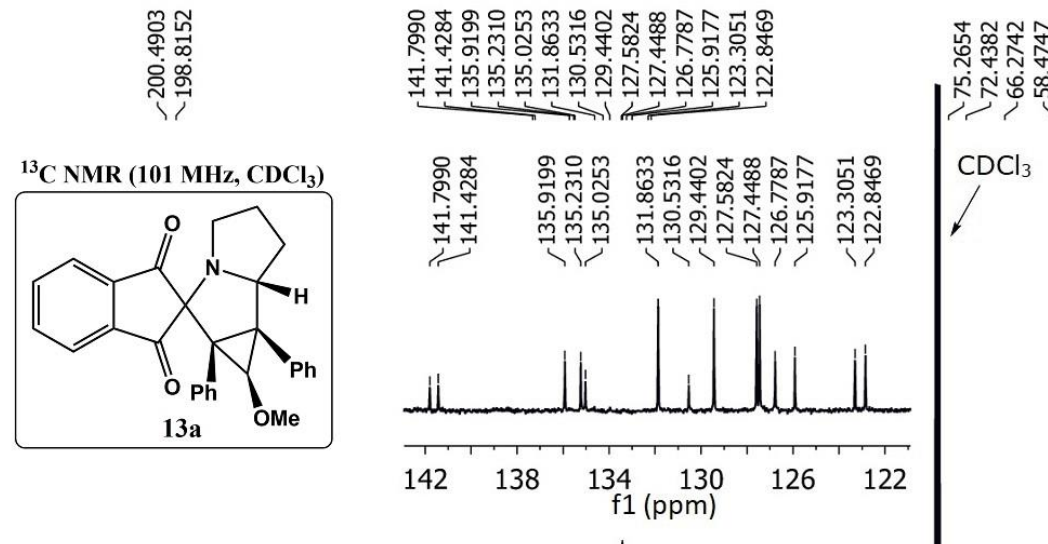

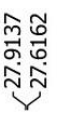

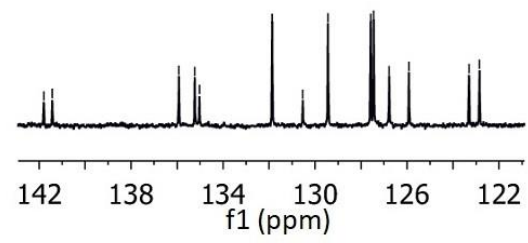

誉

๙ָे

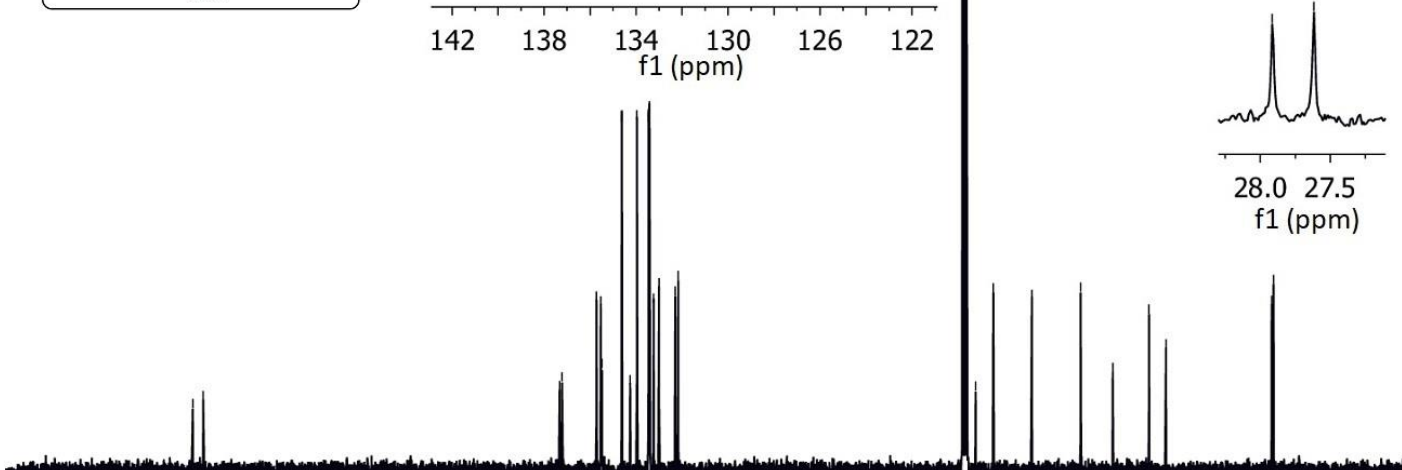

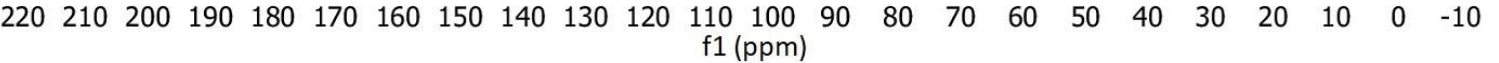

Figure S69. ${ }^{13} \mathrm{C}$ NMR spectrum of compound $\mathbf{1 3 a}\left(\mathrm{CDCl}_{3}, 101 \mathrm{MHz}\right)$ 

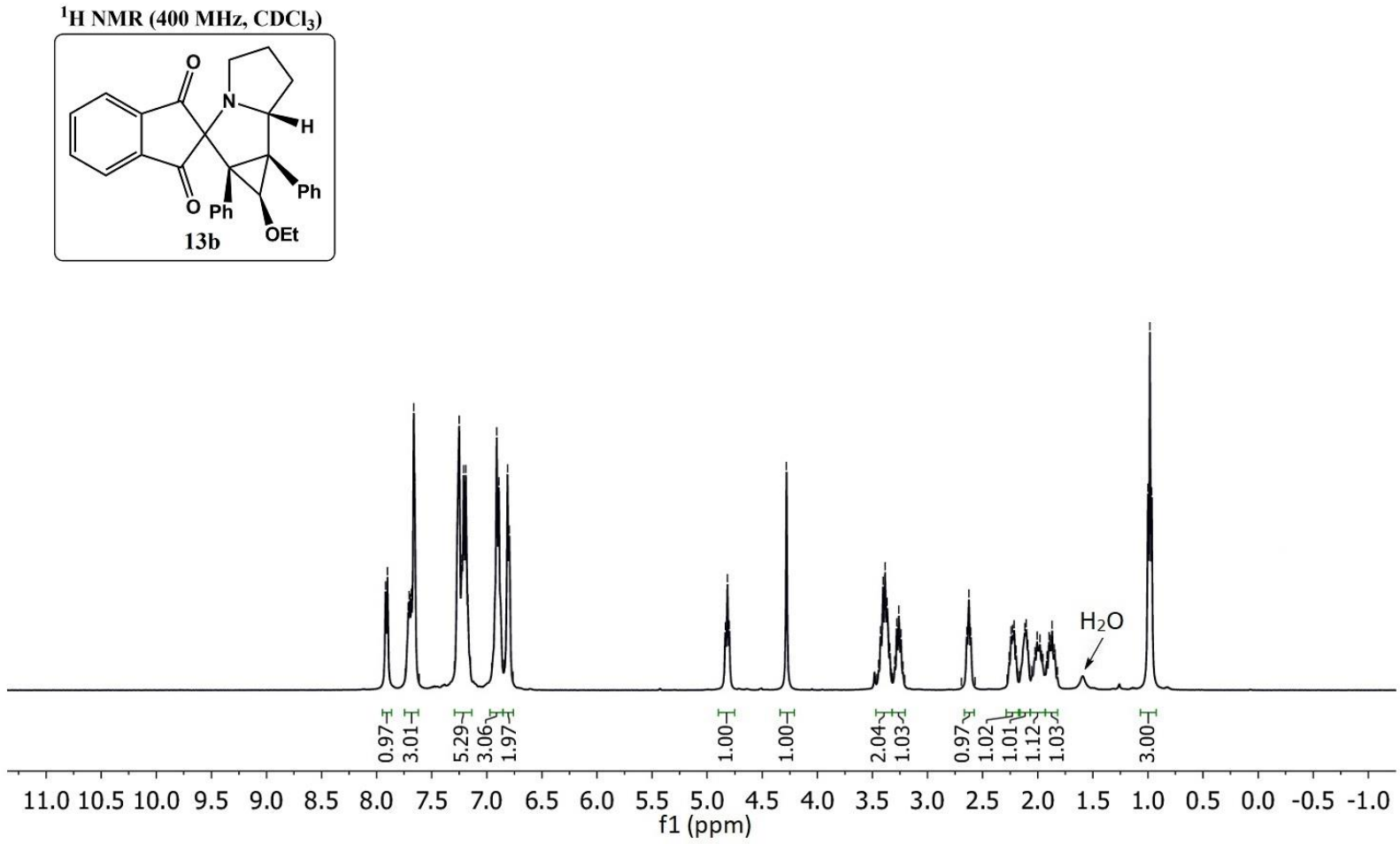

Figure S70. ${ }^{1} \mathrm{H}$ NMR spectrum of compound $\mathbf{1 3 b}\left(\mathrm{CDCl}_{3}, 400 \mathrm{MHz}\right)$

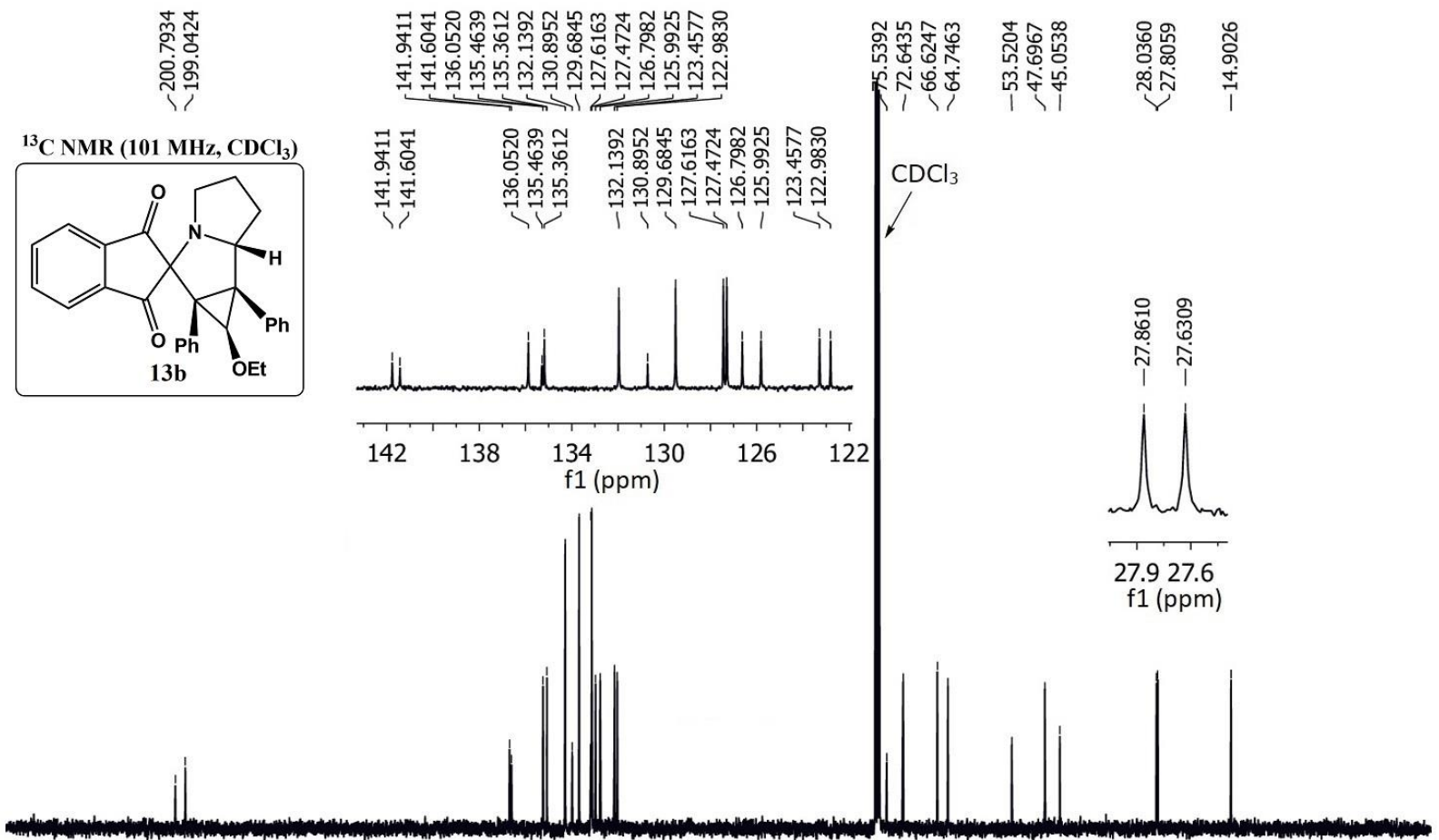

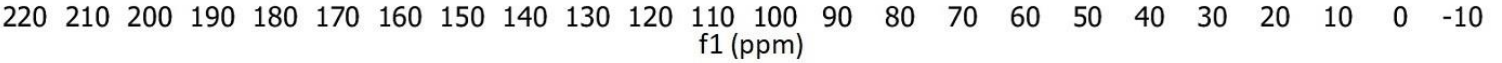

Figure S71. ${ }^{13} \mathrm{C}$ NMR spectrum of compound $\mathbf{1 3 b}\left(\mathrm{CDCl}_{3}, 101 \mathrm{MHz}\right)$ 

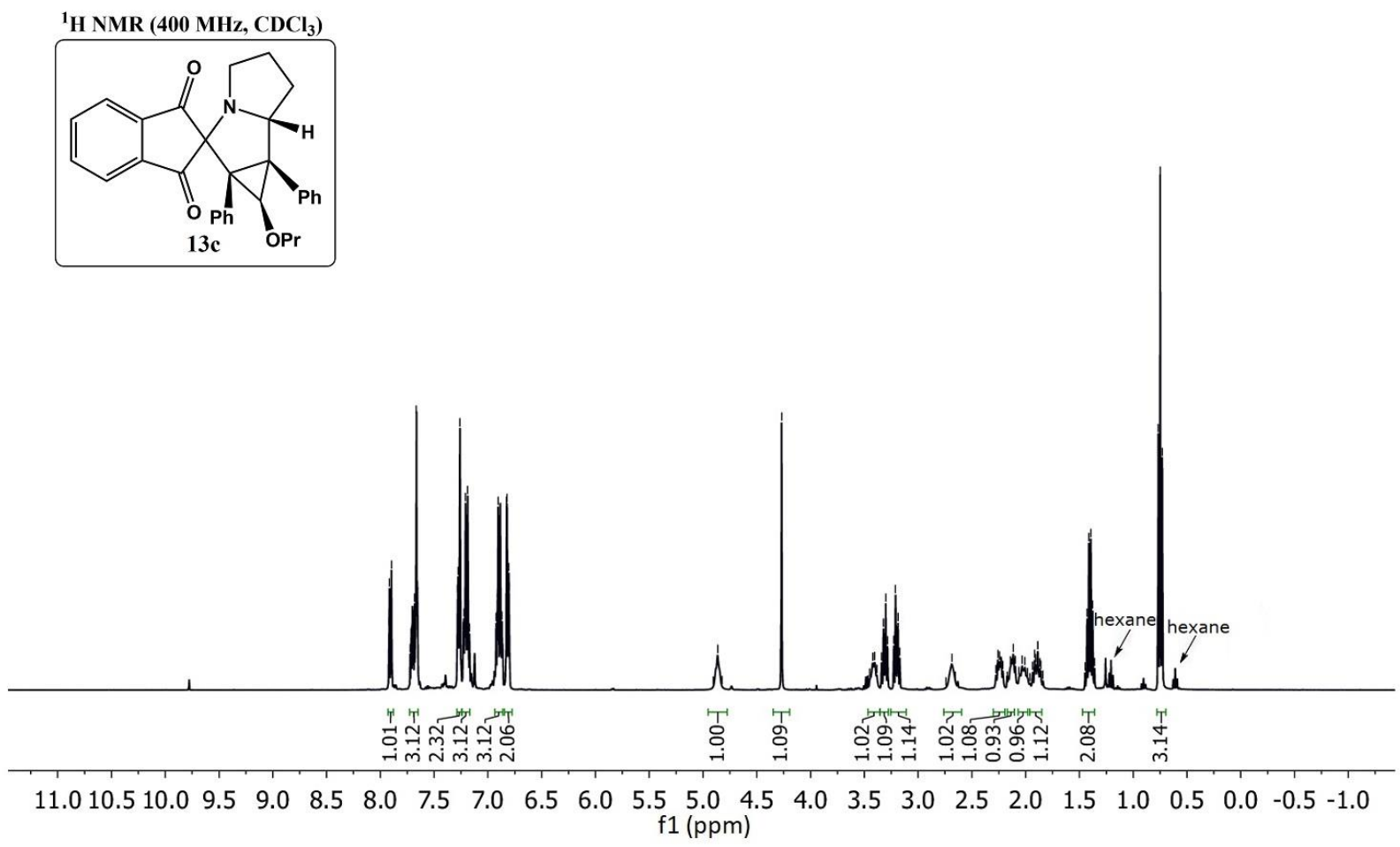

Figure S72. ${ }^{1} \mathrm{H}$ NMR spectrum of compound $13 \mathbf{c}\left(\mathrm{CDCl}_{3}, 400 \mathrm{MHz}\right)$

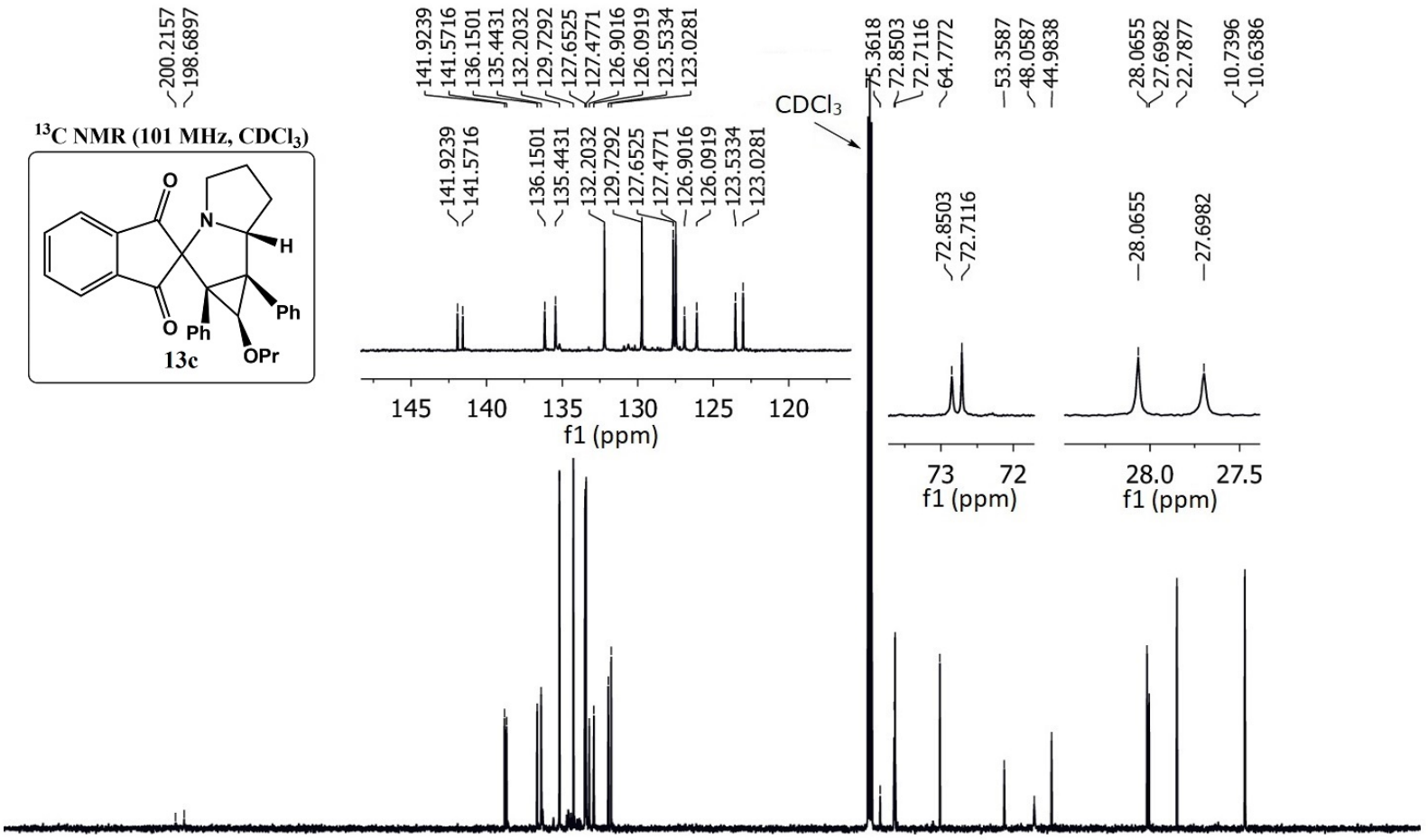

$\begin{array}{llllllllllllllllllllllll}220 & 210 & 200 & 190 & 180 & 170 & 160 & 150 & 140 & 130 & 120 & \begin{array}{l}110 \\ \mathrm{f} 1(\mathrm{ppm})\end{array} & 100 & 80 & 70 & 60 & 50 & 40 & 30 & 20 & 10 & 0 & -10\end{array}$

Figure S73. ${ }^{13} \mathrm{C}$ NMR spectrum of compound $13 \mathrm{c}\left(\mathrm{CDCl}_{3}, 101 \mathrm{MHz}\right)$ 
${ }^{1} \mathrm{H}$ NMR (400 MHz, $\left.\mathrm{CDCl}_{3}\right)$
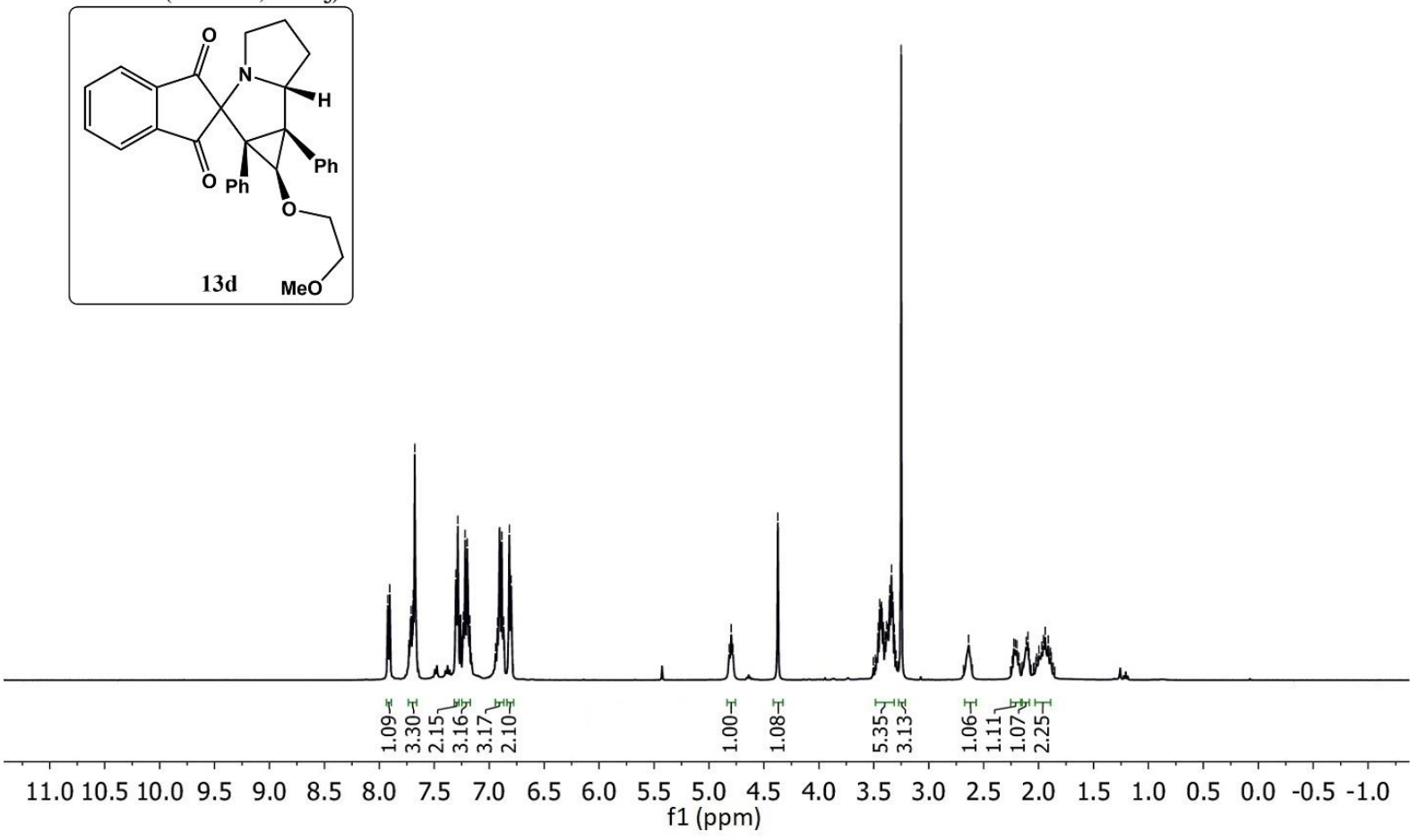

Figure S74. ${ }^{1} \mathrm{H}$ NMR spectrum of compound $\mathbf{1 3 d}\left(\mathrm{CDCl}_{3}, 400 \mathrm{MHz}\right)$

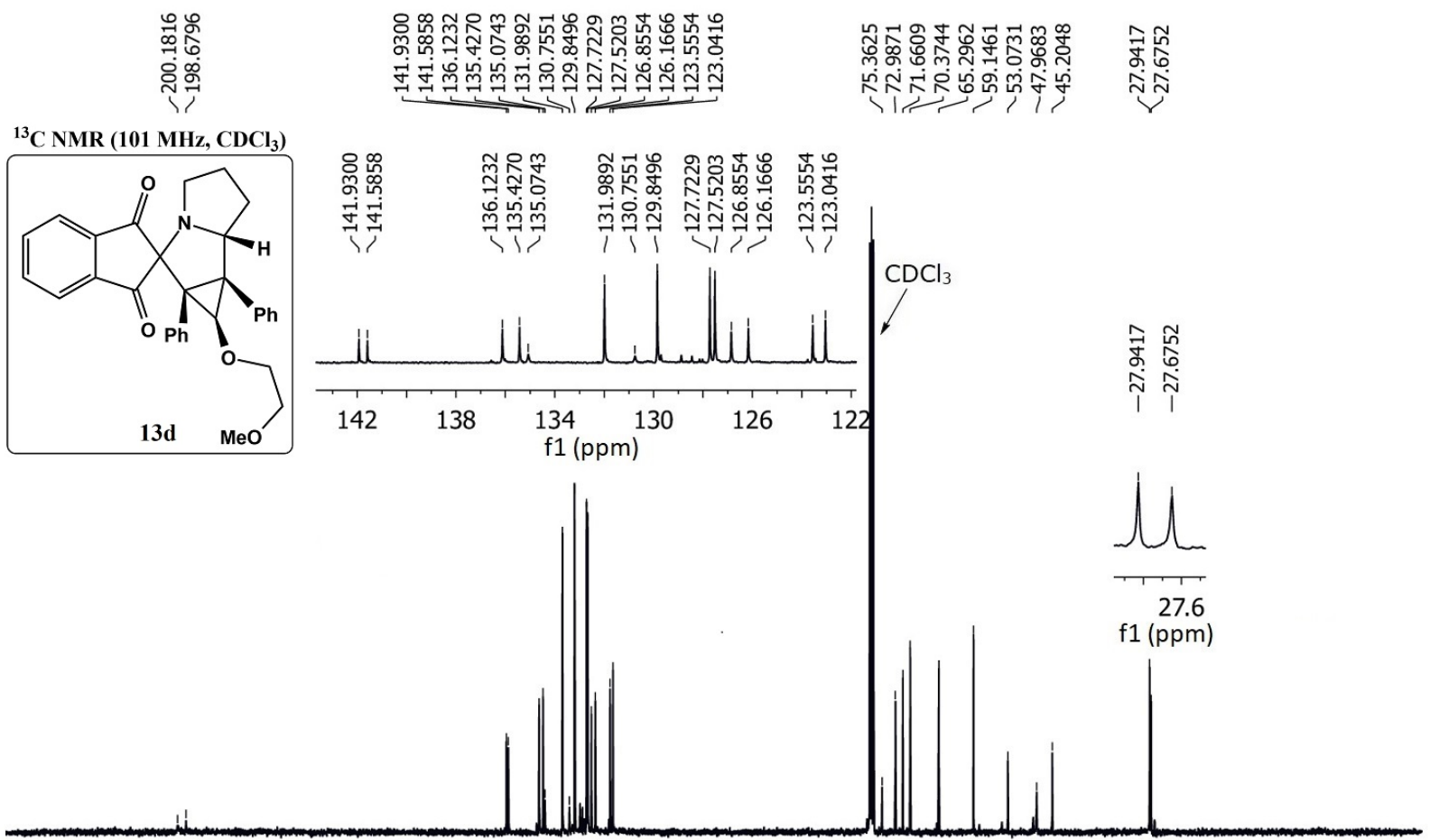

$\begin{array}{llllllllllllllllllllllll}220 & 210 & 200 & 190 & 180 & 170 & 160 & 150 & 140 & 130 & 120 & \begin{array}{c}110 \\ \mathrm{f} 1(\mathrm{ppm})\end{array} & 100 & 90 & 80 & 70 & 60 & 50 & 40 & 30 & 20 & 10 & 0 & -10\end{array}$

Figure S75. ${ }^{13} \mathrm{C}$ NMR spectrum of compound $13 d\left(\mathrm{CDCl}_{3}, 101 \mathrm{MHz}\right)$ 
${ }^{1} \mathrm{H}$ NMR (400 MHz, $\left.\mathrm{CDCl}_{3}\right)$
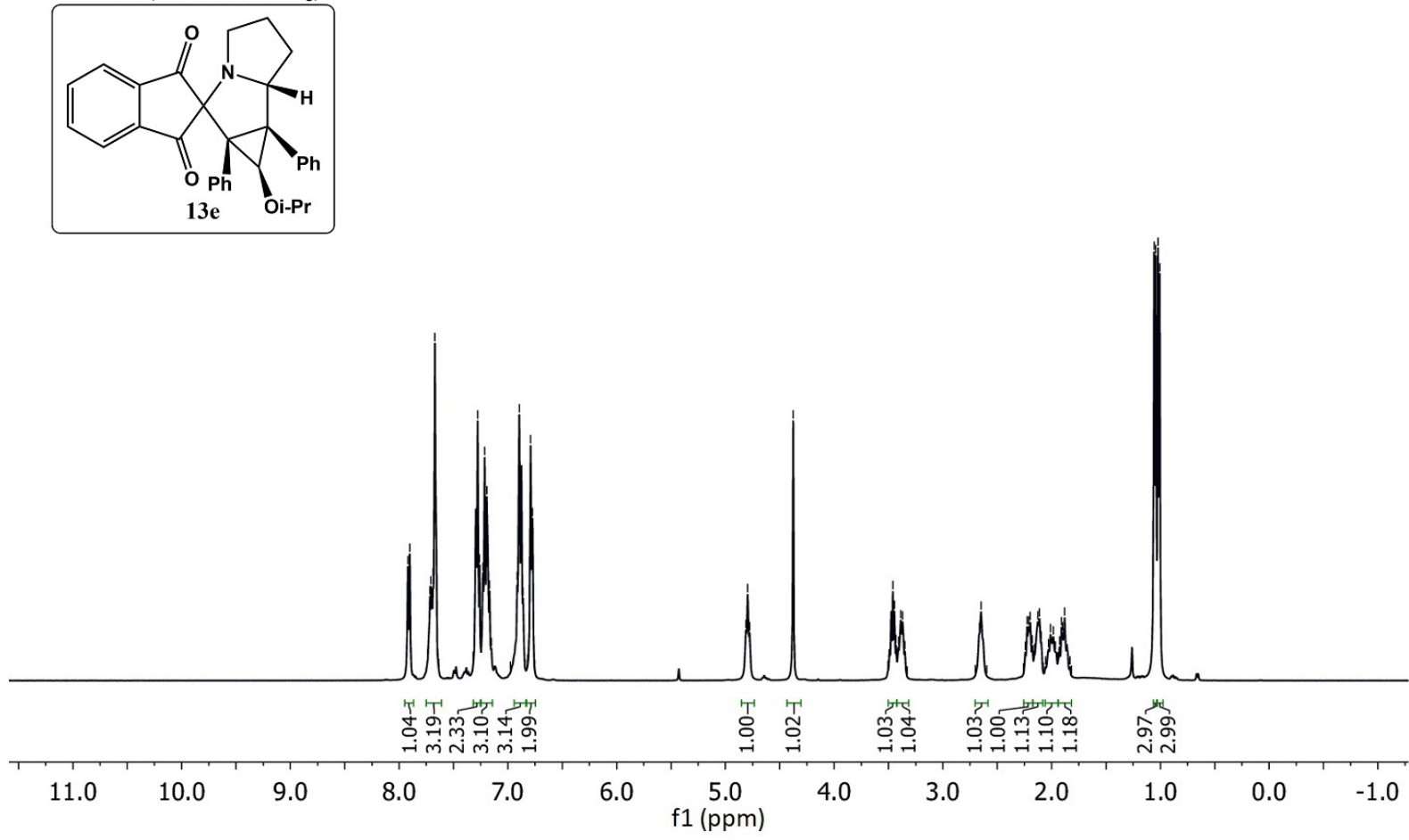

Figure S76. ${ }^{1} \mathrm{H}$ NMR spectrum of compound $13 \mathrm{e}\left(\mathrm{CDCl}_{3}, 400 \mathrm{MHz}\right)$
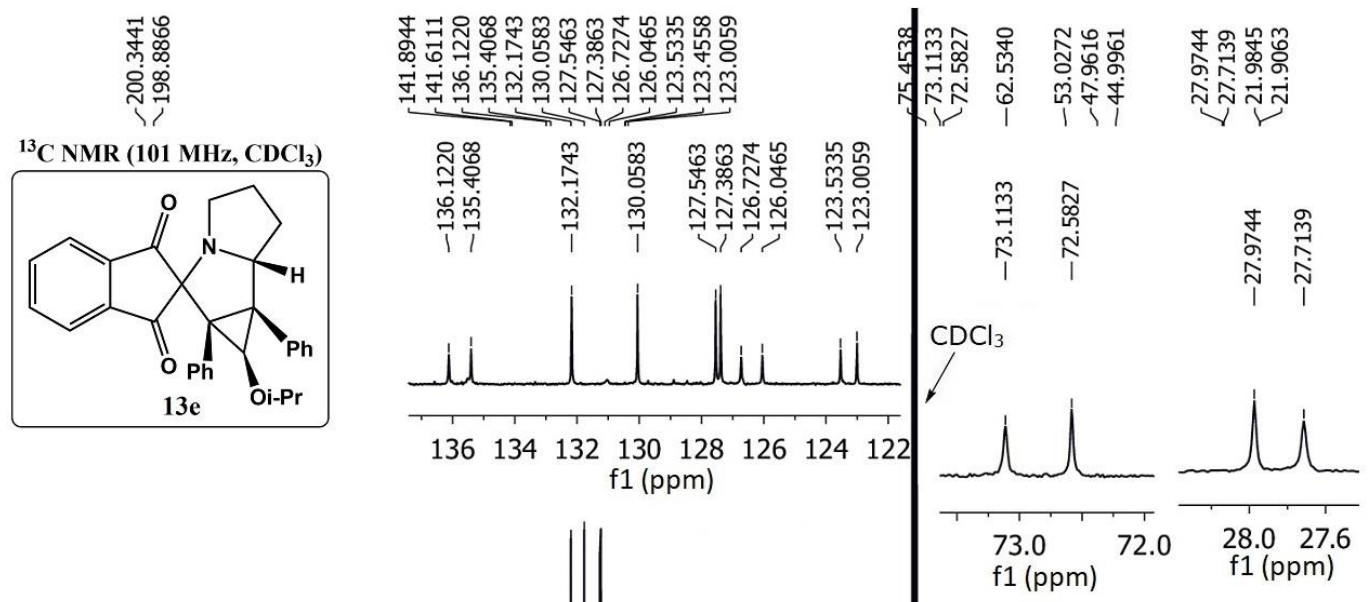

$\begin{array}{llllllllllllllllllllllll}220 & 210 & 200 & 190 & 180 & 170 & 160 & 150 & 140 & 130 & 120 & \begin{array}{c}110 \\ \mathrm{f} 1(\mathrm{ppm})\end{array} & 100 & 90 & 80 & 70 & 60 & 50 & 40 & 30 & 20 & 10 & 0 & -10\end{array}$

Figure S77. ${ }^{13} \mathrm{C}$ NMR spectrum of compound $\mathbf{1 3 e}\left(\mathrm{CDCl}_{3}, 101 \mathrm{MHz}\right)$ 
${ }^{1} \mathrm{H}$ NMR (400 MHz, $\left.\mathrm{CDCl}_{3}\right)$
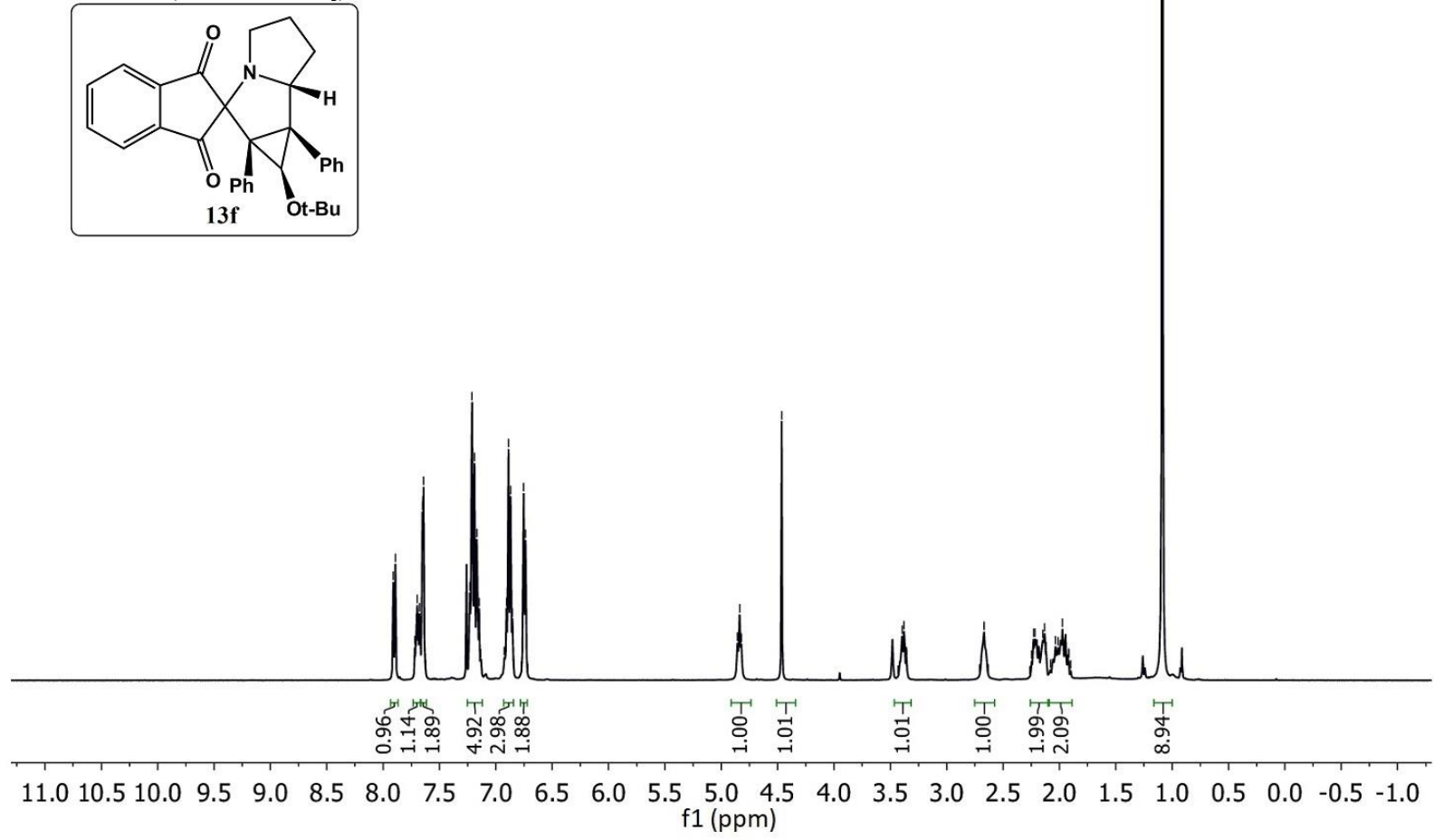

Figure S78. ${ }^{1} \mathrm{H}$ NMR spectrum of compound $\mathbf{1 3 f}\left(\mathrm{CDCl}_{3}, 400 \mathrm{MHz}\right)$
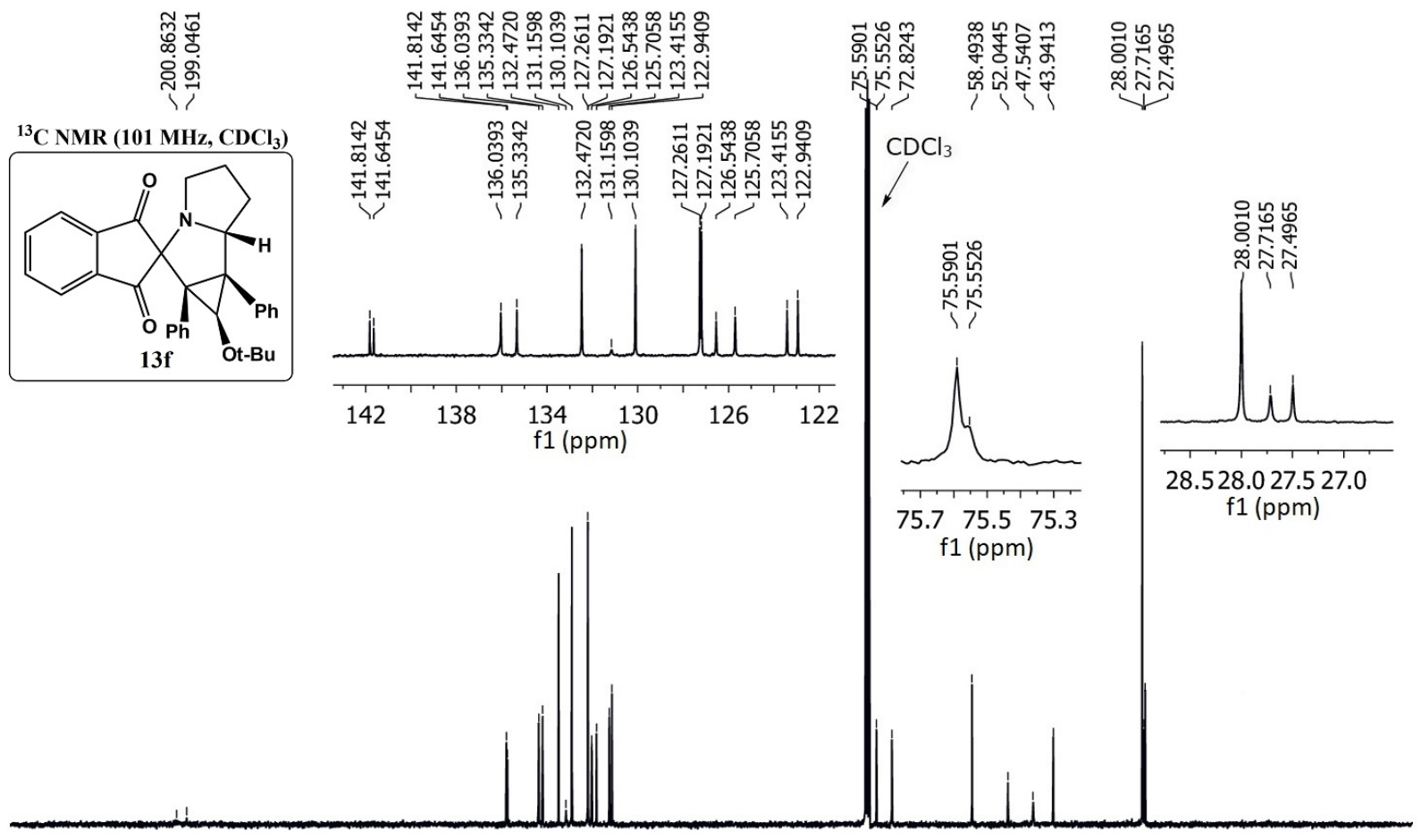

$\begin{array}{llllllllllllllllllllllll}220 & 210 & 200 & 190 & 180 & 170 & 160 & 150 & 140 & 130 & 120 & \begin{array}{l}110 \\ \mathrm{f} 1(\mathrm{ppm})\end{array} & 100 & 90 & 80 & 70 & 60 & 50 & 40 & 30 & 20 & 10 & 0 & -10\end{array}$

Figure S79. ${ }^{13} \mathrm{C}$ NMR spectrum of compound $\mathbf{1 3 f}\left(\mathrm{CDCl}_{3}, 101 \mathrm{MHz}\right)$ 

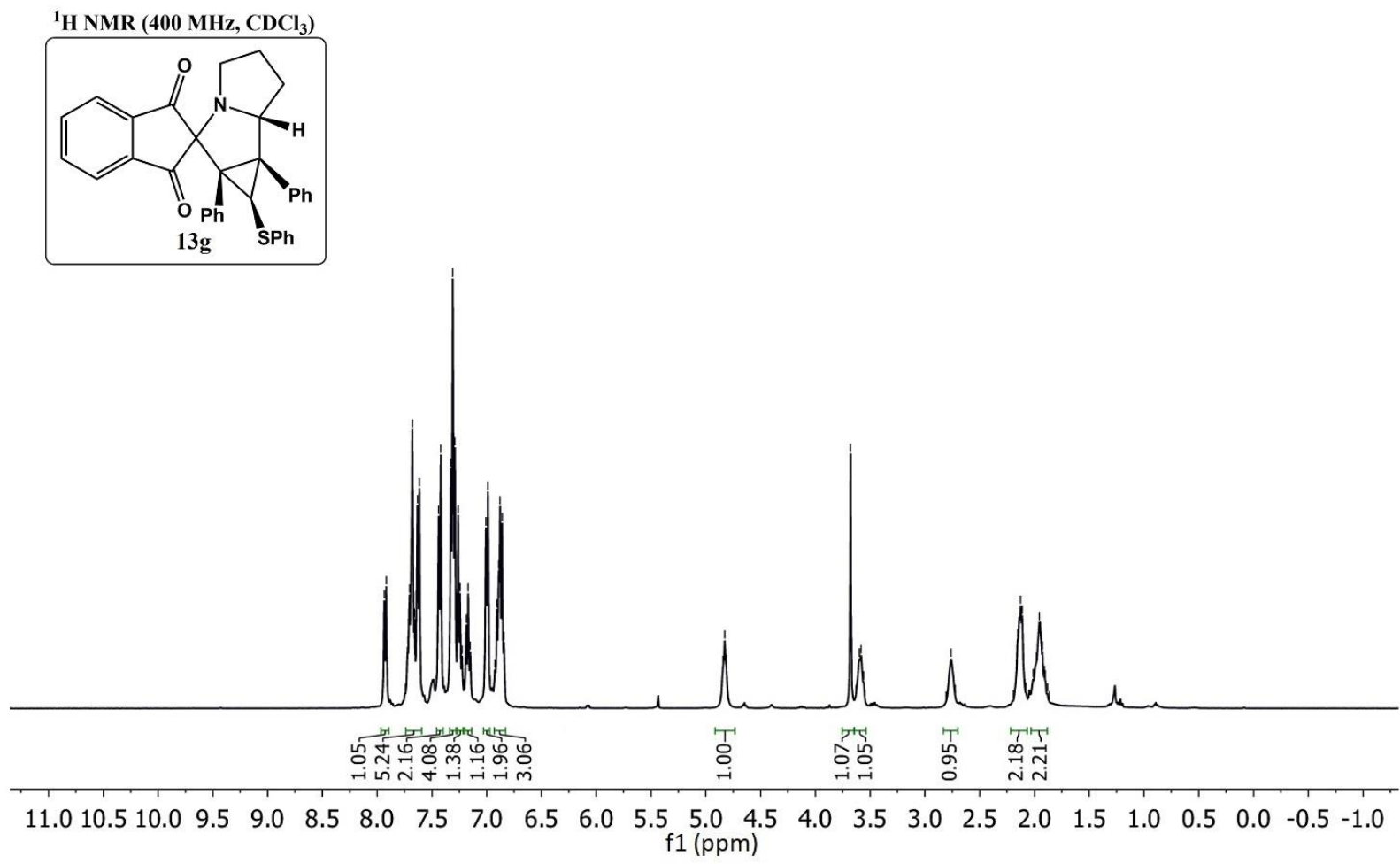

Figure S80. ${ }^{1} \mathrm{H}$ NMR spectrum of compound $\mathbf{1 3 g}\left(\mathrm{CDCl}_{3}, 400 \mathrm{MHz}\right)$

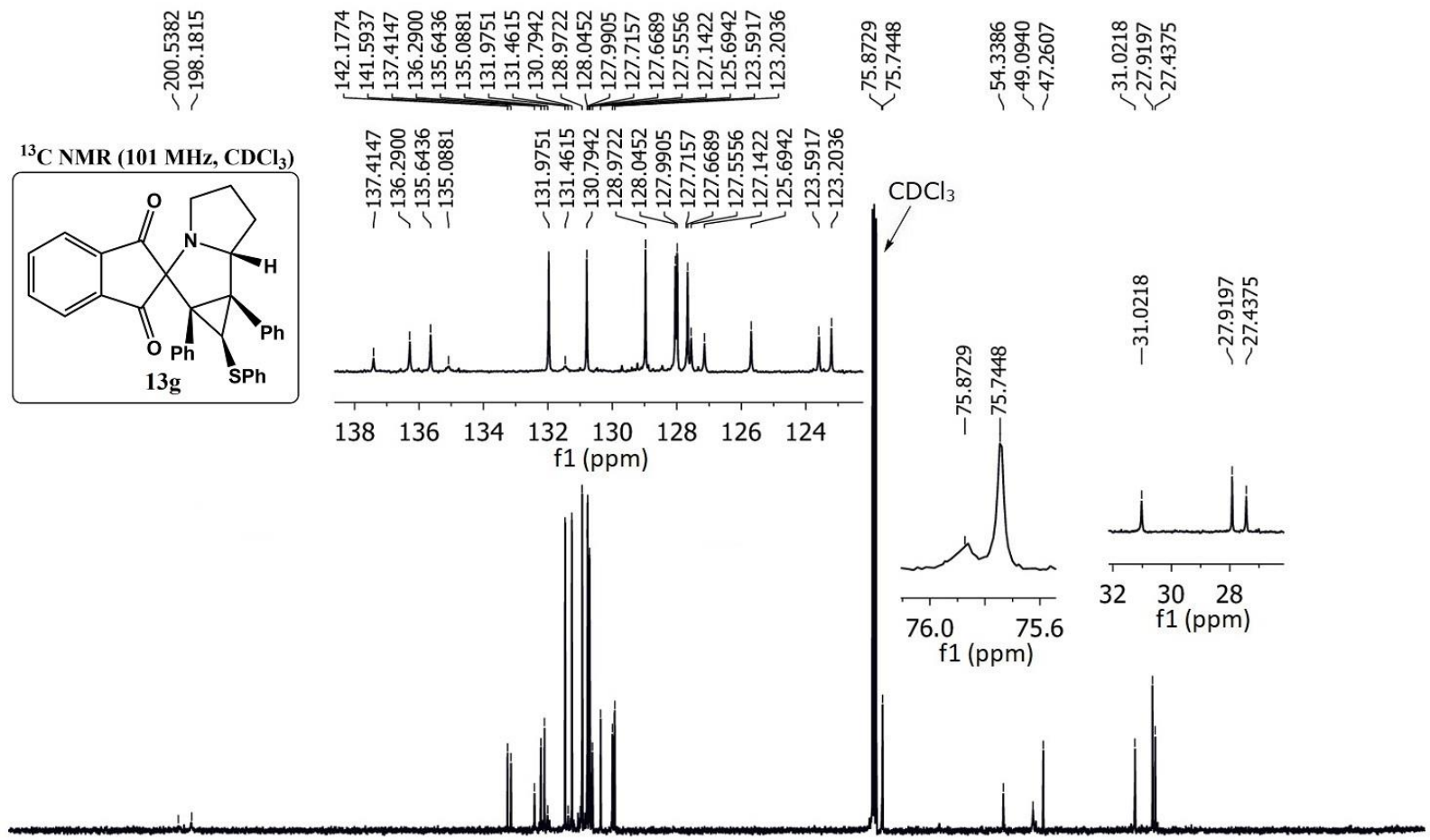

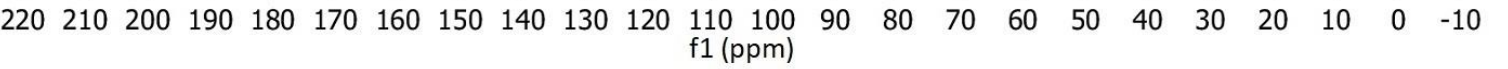

Figure S81. ${ }^{13} \mathrm{C}$ NMR spectrum of compound $13 g\left(\mathrm{CDCl}_{3}, 101 \mathrm{MHz}\right)$ 


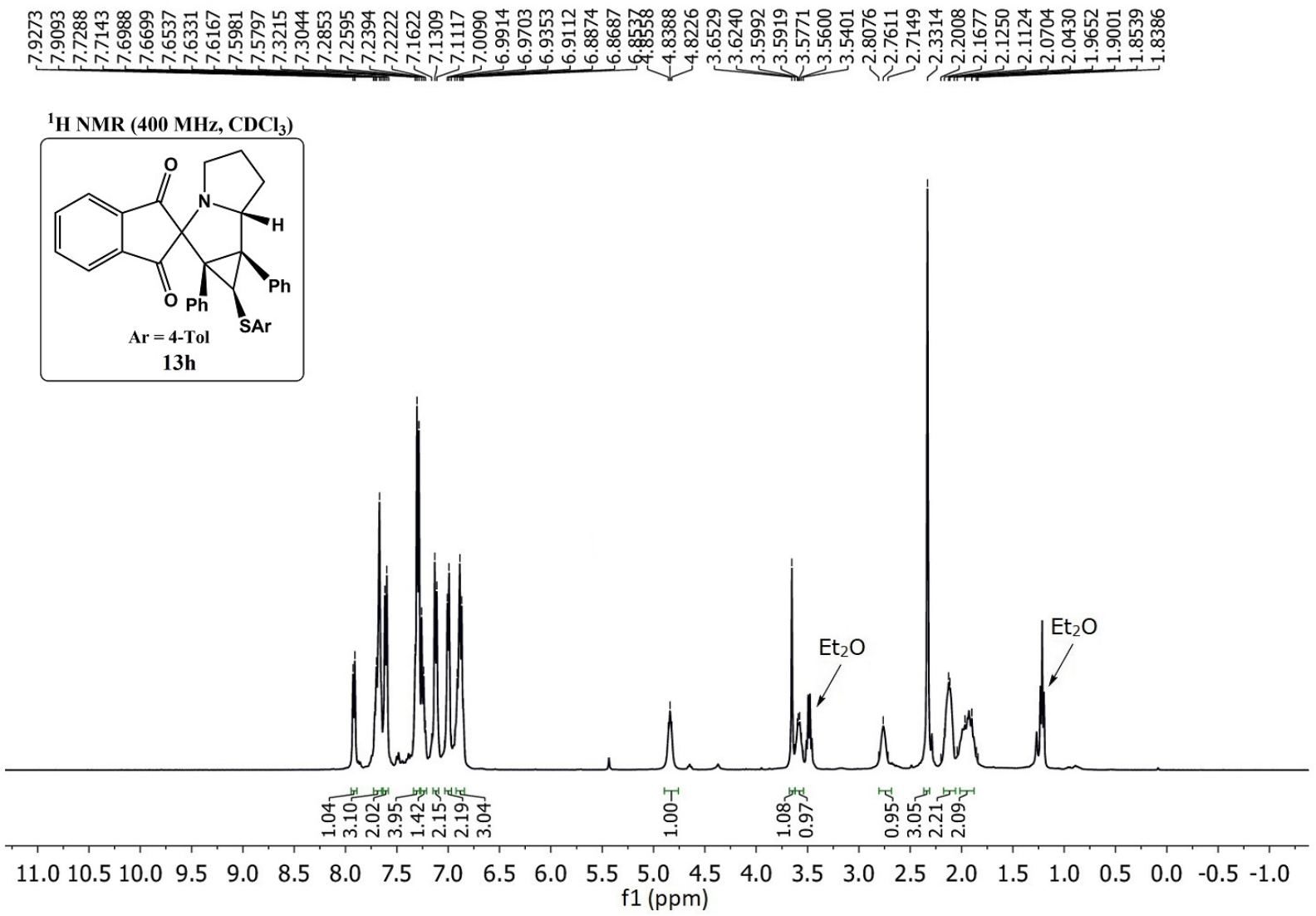

Figure S82. ${ }^{1} \mathrm{H}$ NMR spectrum of compound $\mathbf{1 3 h}\left(\mathrm{CDCl}_{3}, 400 \mathrm{MHz}\right)$

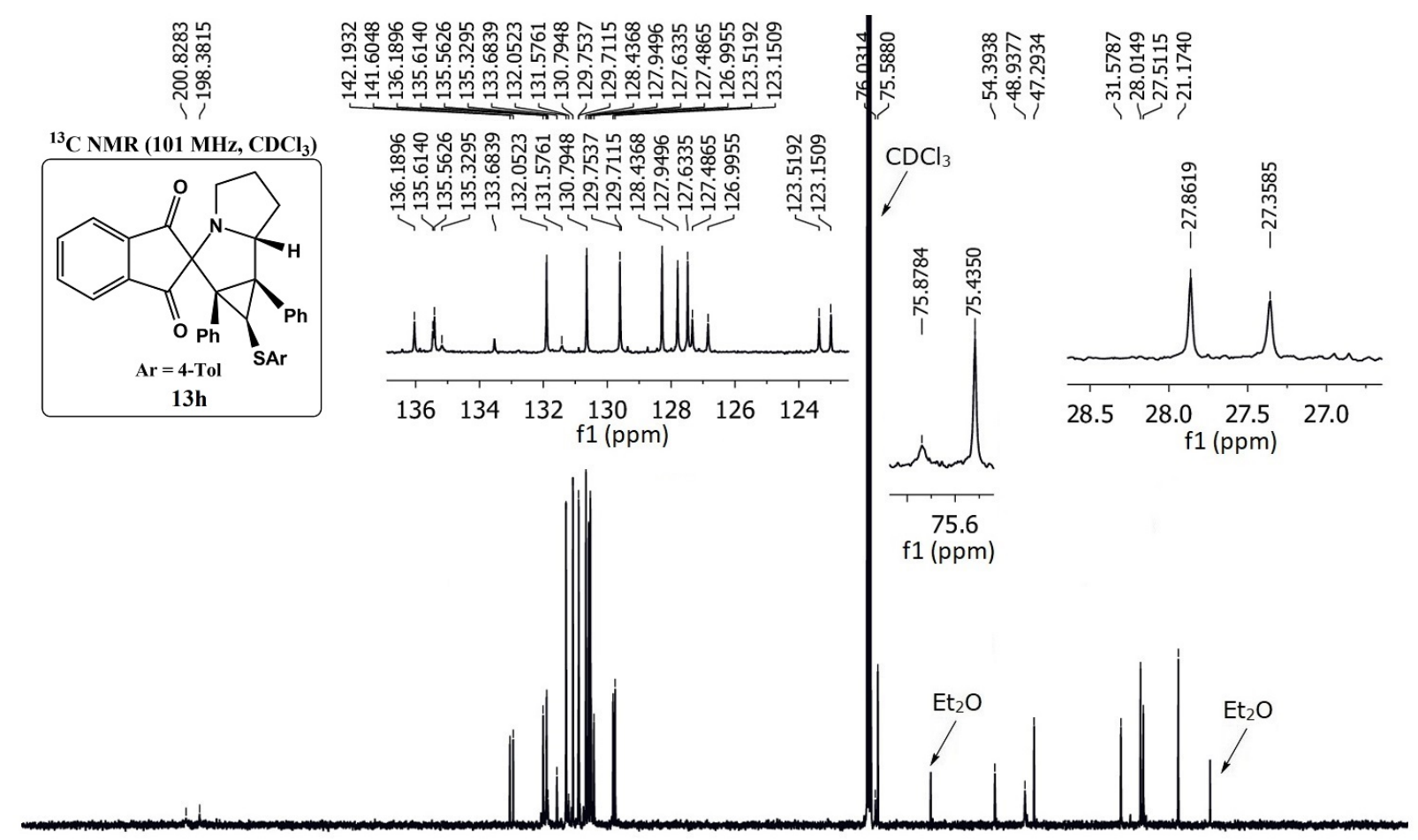

$\begin{array}{llllllllllllllllllllllll}220 & 210 & 200 & 190 & 180 & 170 & 160 & 150 & 140 & 130 & 120 & \begin{array}{l}110 \\ \mathrm{f} 1(\mathrm{ppm})\end{array} & 100 & 90 & 80 & 70 & 60 & 50 & 40 & 30 & 20 & 10 & 0 & -10\end{array}$

Figure S83. ${ }^{13} \mathrm{C}$ NMR spectrum of compound $\mathbf{1 3 h}\left(\mathrm{CDCl}_{3}, 101 \mathrm{MHz}\right)$ 
${ }^{1} \mathrm{H}$ NMR (400 MHz, $\left.\mathrm{CDCl}_{3}\right)$
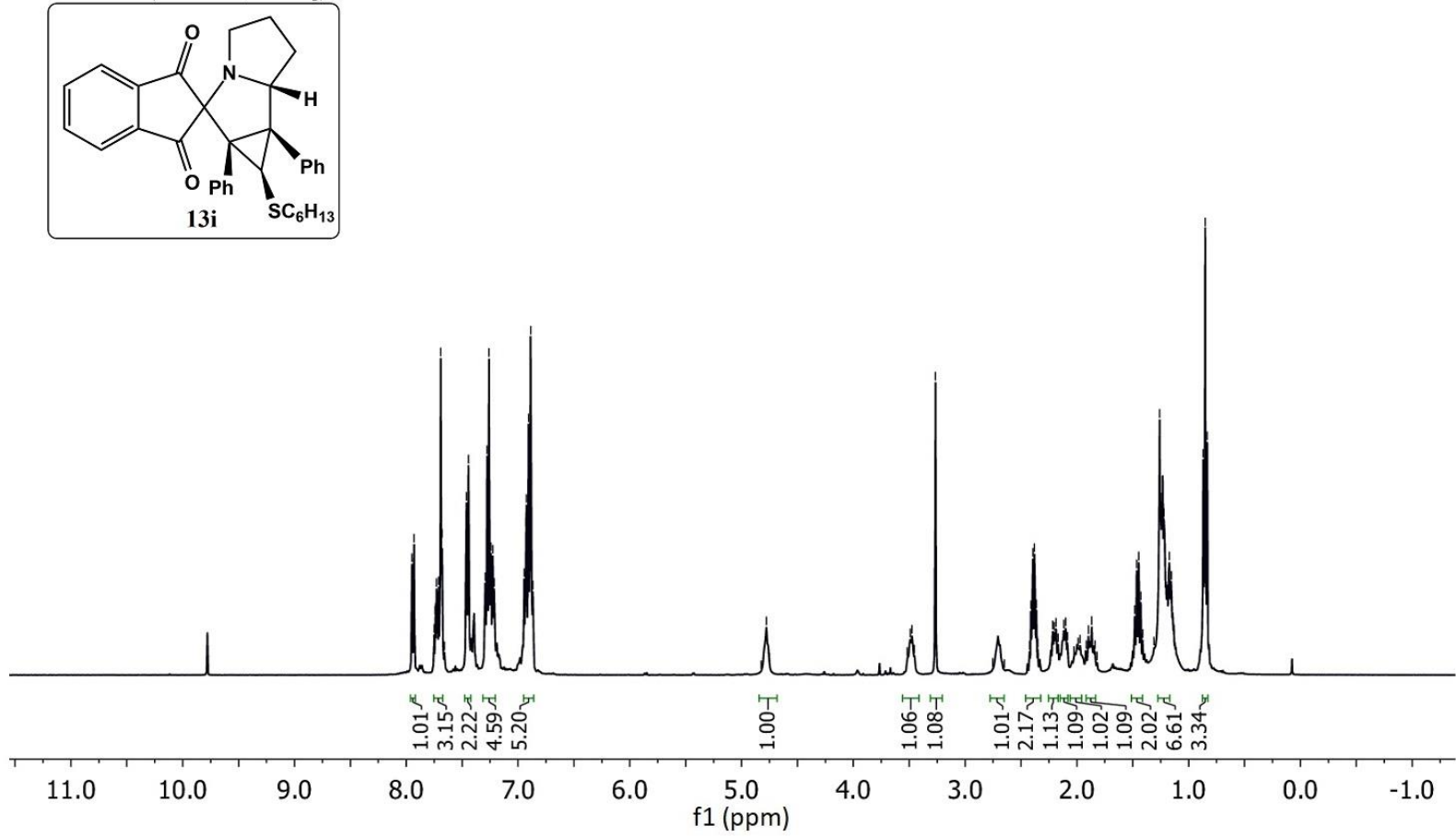

Figure S84. ${ }^{1} \mathrm{H}$ NMR spectrum of compound $13 i\left(\mathrm{CDCl}_{3}, 400 \mathrm{MHz}\right)$
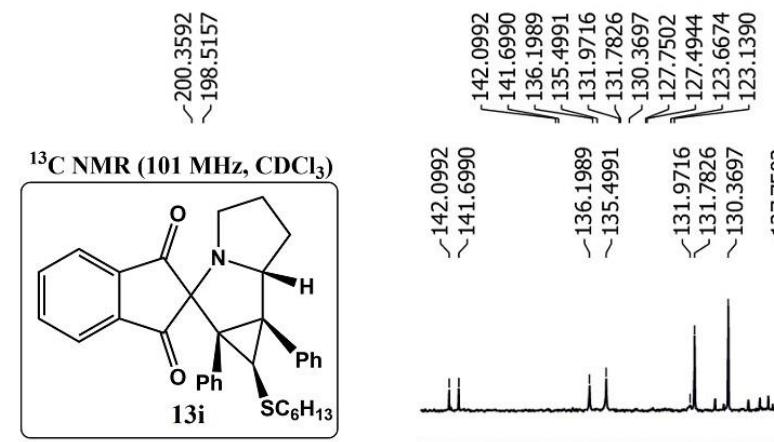

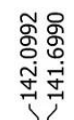

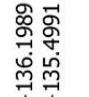

गें
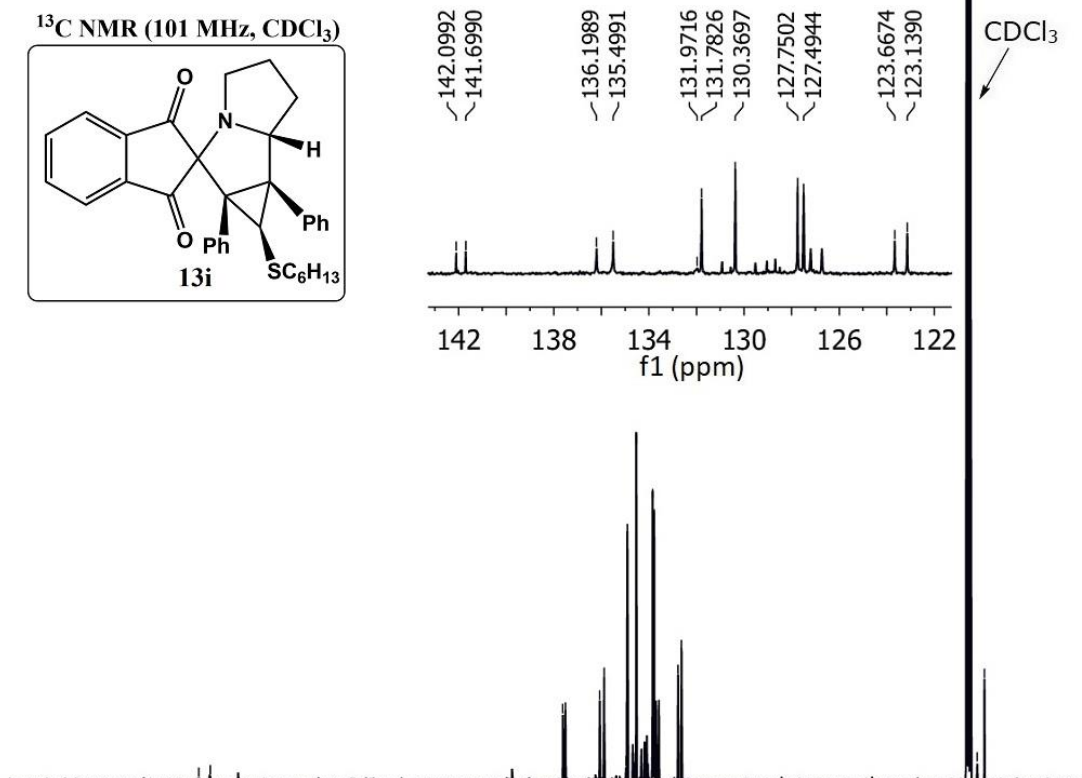

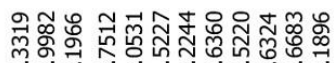

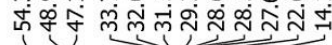
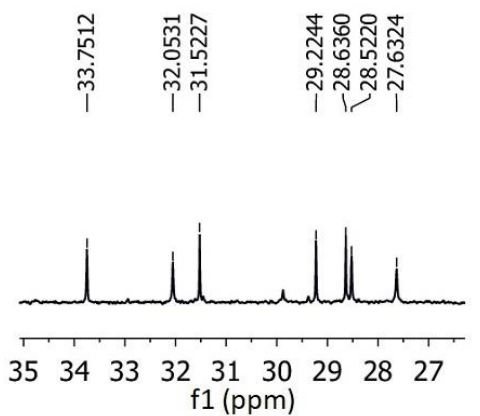

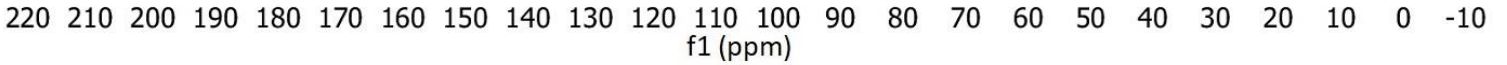

Figure S85. ${ }^{13} \mathrm{C}$ NMR spectrum of compound $\mathbf{1 3 i}\left(\mathrm{CDCl}_{3}, 101 \mathrm{MHz}\right)$ 
${ }^{1} \mathrm{H}$ NMR (400 MHz, $\left.\mathrm{CDCl}_{3}\right)$
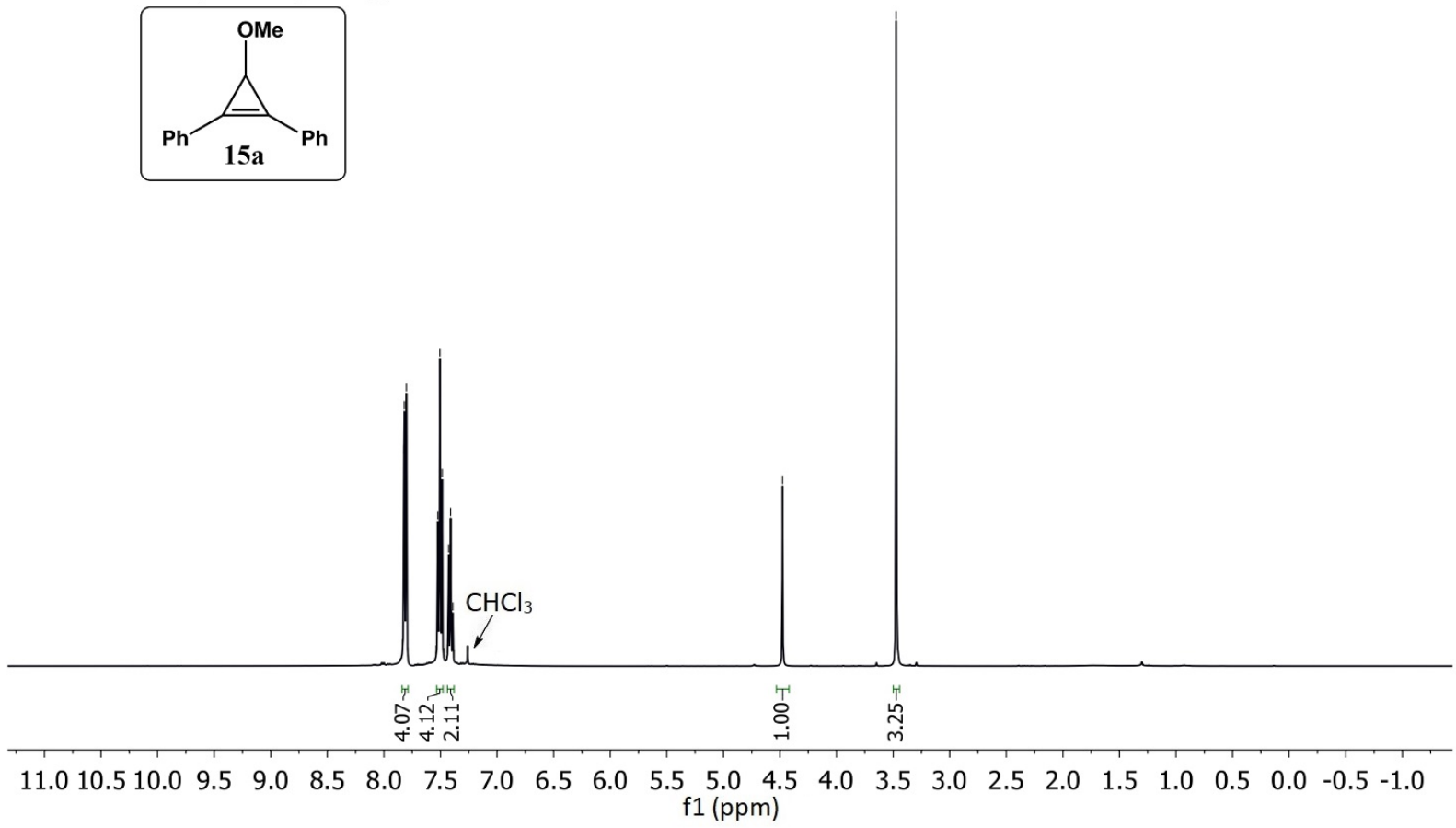

Figure S86. ${ }^{1} \mathrm{H}$ NMR spectrum of compound $\mathbf{1 5 a}\left(\mathrm{CDCl}_{3}, 400 \mathrm{MHz}\right)$

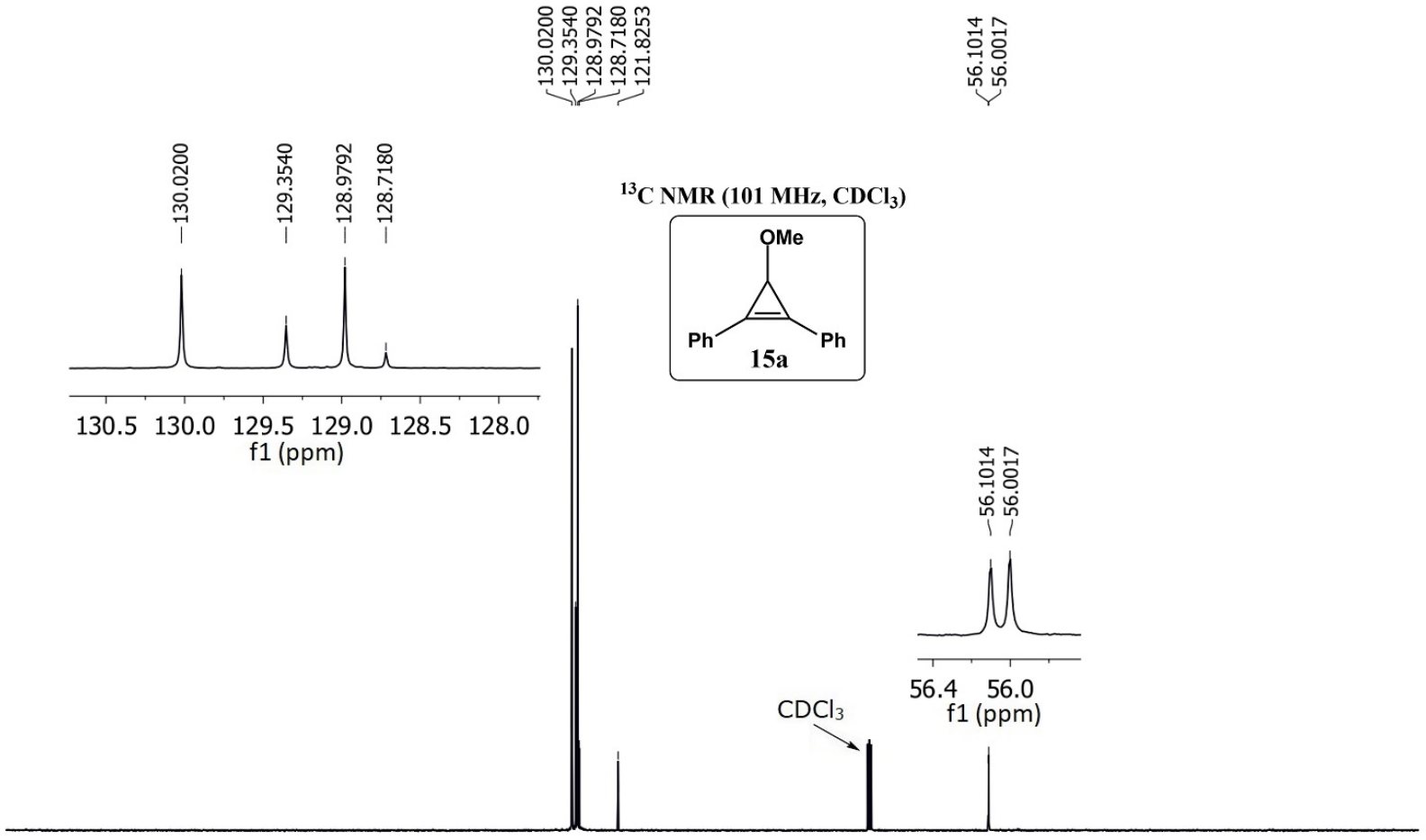

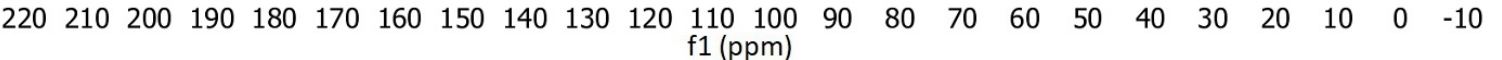

Figure S87. ${ }^{13} \mathrm{C}$ NMR spectrum of compound $\mathbf{1 5 a}\left(\mathrm{CDCl}_{3}, 101 \mathrm{MHz}\right)$ 


\section{X-ray crystallographic data for compounds $7 \mathrm{a}, 11 \mathrm{a}, 11 \mathrm{~h}$ and $13 \mathrm{~b}$}

General procedure of the sample preparation and crystal structure determination: Single crystals of compounds $7 \mathbf{a}, 11 \mathbf{a}, 11 \mathbf{h}$ and $13 \mathbf{b}$ were growth by slow evaporation of their solutions in an ethanol-chloroform mixture at room temperature. For single crystal X-ray diffraction experiment crystals were fixed on a micro mount and placed on at SuperNova, single source at offset/far, HyPix3000 or Xcalibur Eos diffractometers and were measured at $100 \mathrm{~K}$ using monochromated $\mathrm{CuK} \alpha(\mathbf{7 a}, \mathbf{1 1 h}$ and $\mathbf{1 3 b})$ and $\mathrm{MoK} \alpha(\mathbf{1 1 a})$ radiations, respectively. The structures have been solved by the ShelXT ${ }^{1}$ structure solution program using Intrinsic Phasing and the Superflip ${ }^{2}$ structure solution program using Charge Flipping and refined by means of the SHELXL program $^{3}$ incorporated in the OLEX2 program package. ${ }^{4}$ Empirical absorption correction was applied in CrysAlisPro program complex ${ }^{5,6}$ using spherical harmonics, implemented in SCALE3 ABSPACK scaling algorithm. The crystallographic data and some parameters of refinement are placed in Tables S1-S4. 


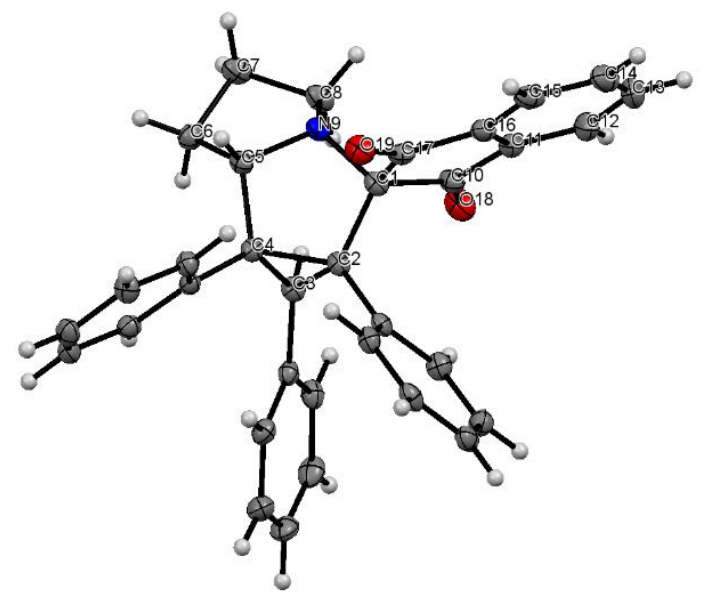

Figure S88. ORTEP representation of the molecular structure of 7a (CCDC-1841118).

Thermal ellipsoids are drawn at 50\% probability level.

Table S1. Crystal data and structure refinement for compound 7a

Empirical formula

Formula weight

Temperature/K

Crystal system

Space group

$\mathrm{a} / \AA$

$\mathrm{b} / \AA$

$\mathrm{c} / \AA$

$\alpha /{ }^{\circ}$

$\beta /{ }^{\circ}$

$\gamma /{ }^{\circ}$

Volume $/ \AA^{3}$

$\mathrm{Z}$

$\rho_{\text {calc }} / \mathrm{cm}^{3}$

$\mu / \mathrm{mm}^{-1}$

$\mathrm{F}()$

Crystal size $/ \mathrm{mm}^{3}$

Radiation

$2 \Theta$ range for data collection ${ }^{\circ}$

Index ranges

Reflections collected

Independent reflections

Data/restraints/parameters

Goodness-of-fit on $\mathrm{F}^{2}$

Final $\mathrm{R}$ indexes $[\mathrm{I}>=2 \sigma(\mathrm{I})]$

Final $\mathrm{R}$ indexes [all data]

Largest diff. peak/hole / e $\AA^{-3}$
$\mathrm{C}_{34} \mathrm{H}_{27} \mathrm{NO}_{2}$

481.56

100(2)

Orthorhombic

Pbca

$15.2452(3)$

$17.0853(3)$

$19.0868(3)$

90

90

90

4971.51(16)

8

1.287

0.620

2032.0

$0.6 \times 0.5 \times 0.4$

$\mathrm{CuK} \alpha(\lambda=1.54184)$

9.05 to 144.082

$-18 \leq \mathrm{h} \leq 12,-21 \leq \mathrm{k} \leq 21,-23 \leq 1 \leq$ 23

27282

$4852\left[\mathrm{R}_{\text {int }}=0.0426, \mathrm{R}_{\text {sigma }}=0.0230\right]$

$4852 / 0 / 334$

1.031

$\mathrm{R}_{1}=0.0370, \mathrm{wR}_{2}=0.0918$

$\mathrm{R}_{1}=0.0392, \mathrm{wR}_{2}=0.0935$

$0.24 /-0.24$ 


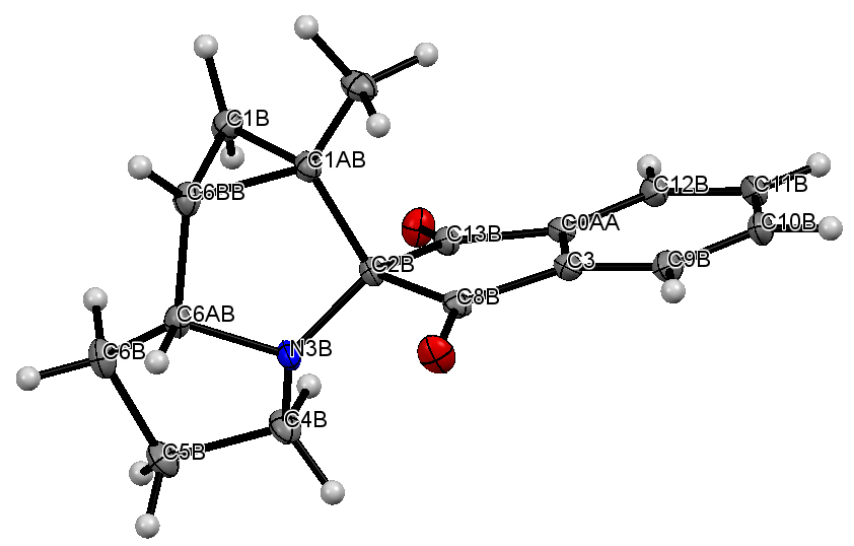

Figure S89. ORTEP representation of the molecular structure of 11a (CCDC-1897279).

Thermal ellipsoids are drawn at 50\% probability level.

Table S2. Crystal data and structure refinement for compound 11a

\begin{tabular}{|c|c|}
\hline Empirical formula & $\mathrm{C}_{17} \mathrm{H}_{17} \mathrm{NO}_{2}$ \\
\hline Formula weight & 267.31 \\
\hline Temperature/K & $100(2)$ \\
\hline Crystal system & Triclinic \\
\hline Space group & $\mathrm{P}-1$ \\
\hline $\mathrm{a} / \AA ̊$ & $9.7016(4)$ \\
\hline $\mathrm{b} / \AA$ & $11.6604(4)$ \\
\hline $\mathrm{c} / \AA$ & $11.7270(5)$ \\
\hline$\alpha /^{\circ}$ & $88.483(3)$ \\
\hline$\beta /{ }^{\circ}$ & $86.881(3)$ \\
\hline$\gamma /{ }^{\circ}$ & $89.643(3)$ \\
\hline Volume $/ \AA^{3}$ & $1324.16(9)$ \\
\hline $\mathrm{Z}$ & 4 \\
\hline$\rho_{\text {calc }} \mathrm{g} / \mathrm{cm}^{3}$ & 1.341 \\
\hline$\mu / \mathrm{mm}^{-1}$ & 0.088 \\
\hline $\mathrm{F}()$ & 568.0 \\
\hline Crystal size $/ \mathrm{mm}^{3}$ & $0.46 \times 0.4 \times 0.29$ \\
\hline Radiation & $\operatorname{MoK} \alpha(\lambda=0.71073)$ \\
\hline $2 \Theta$ range for data collection ${ }^{\circ}$ & 5.456 to 55 \\
\hline Index ranges & $-12 \leq \mathrm{h} \leq 12,-15 \leq \mathrm{k} \leq 15,-15 \leq 1 \leq 15$ \\
\hline Reflections collected & 21722 \\
\hline Independent reflections & $6082\left[\mathrm{R}_{\mathrm{int}}=0.0315, \mathrm{R}_{\text {sigma }}=0.0308\right]$ \\
\hline Data/restraints/parameters & $6082 / 0 / 363$ \\
\hline Goodness-of-fit on $\mathrm{F}^{2}$ & 1.101 \\
\hline Final $R$ indexes $[\mathrm{I}>=2 \sigma(\mathrm{I})]$ & $\mathrm{R}_{1}=0.0721, \mathrm{wR}_{2}=0.1929$ \\
\hline Final $\mathrm{R}$ indexes [all data] & $\mathrm{R}_{1}=0.0791, \mathrm{wR}_{2}=0.1984$ \\
\hline Largest diff. peak/hole / e $\AA^{-3}$ & $0.72 /-0.31$ \\
\hline
\end{tabular}




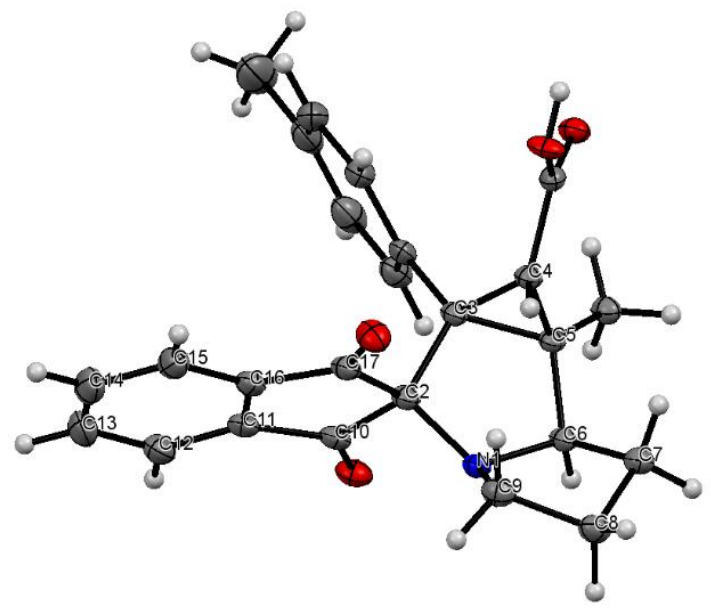

Figure S90. ORTEP representation of the molecular structure of 11h (CCDC-1843132). Thermal ellipsoids are drawn at 50\% probability level.

Table S3. Crystal data and structure refinement for compound $\mathbf{1 1 h}$

\begin{tabular}{|c|c|}
\hline Empirical formula & $\mathrm{C}_{25} \mathrm{H}_{23} \mathrm{NO}_{4}$ \\
\hline Formula weight & 401.44 \\
\hline Temperature/K & $100(2)$ \\
\hline Crystal system & Monoclinic \\
\hline Space group & $\mathrm{P} 2{ }_{1} / \mathrm{c}$ \\
\hline $\mathrm{a} / \AA ̊$ & $12.9200(4)$ \\
\hline $\mathrm{b} / \AA$ & $10.2331(3)$ \\
\hline $\mathrm{c} / \AA$ & $15.3199(4)$ \\
\hline$\alpha /^{\circ}$ & 90 \\
\hline$\beta /{ }^{\circ}$ & $99.360(3)$ \\
\hline$\gamma /{ }^{\circ}$ & 90 \\
\hline Volume $/ \AA^{3}$ & $1998.50(11)$ \\
\hline $\mathrm{Z}$ & 4 \\
\hline$\rho_{\text {calc }} g / \mathrm{cm}^{3}$ & 1.334 \\
\hline$\mu / \mathrm{mm}^{-1}$ & 0.731 \\
\hline $\mathrm{F}()$ & 848.0 \\
\hline Crystal size $/ \mathrm{mm}^{3}$ & $0.32 \times 0.15 \times 0.15$ \\
\hline Radiation & $\mathrm{CuK} \alpha(\lambda=1.54184)$ \\
\hline $2 \Theta$ range for data collection ${ }^{\circ}$ & 6.934 to 143.87 \\
\hline Index ranges & $-15 \leq \mathrm{h} \leq 15,-12 \leq \mathrm{k} \leq 12,-18 \leq 1 \leq 13$ \\
\hline Reflections collected & 24262 \\
\hline Independent reflections & $3915\left[\mathrm{R}_{\text {int }}=0.0438, \mathrm{R}_{\text {sigma }}=0.0235\right]$ \\
\hline Data/restraints/parameters & $3915 / 0 / 274$ \\
\hline Goodness-of-fit on $\mathrm{F}^{2}$ & 1.053 \\
\hline Final $R$ indexes $[\mathrm{I}>=2 \sigma(\mathrm{I})]$ & $\mathrm{R}_{1}=0.0435, \mathrm{wR}_{2}=0.1081$ \\
\hline Final $\mathrm{R}$ indexes [all data] & $\mathrm{R}_{1}=0.0464, \mathrm{wR}_{2}=0.1101$ \\
\hline Largest diff. peak/hole / e $\AA^{-3}$ & $0.55 /-0.24$ \\
\hline
\end{tabular}




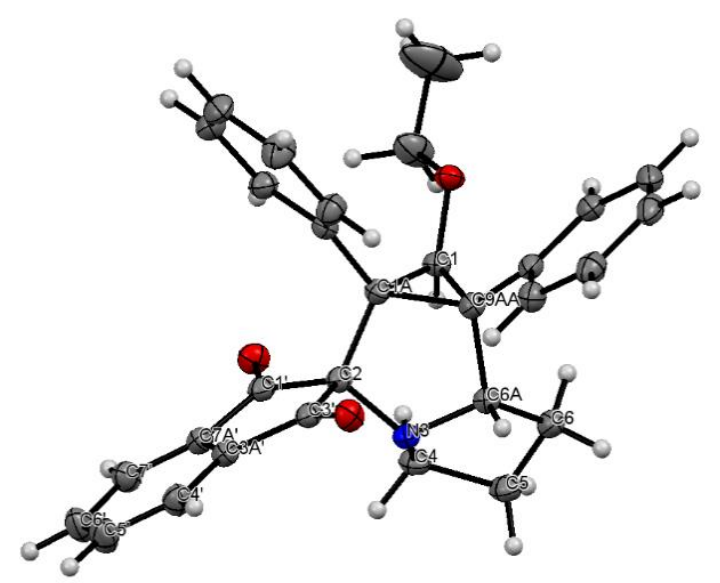

Figure S91. ORTEP representation of the molecular structure of 13b (CCDC-1843131). Thermal ellipsoids are drawn at 50\% probability level.

Table S4. Crystal data and structure refinement for compound $\mathbf{1 3 b}$

\begin{tabular}{|c|c|}
\hline Empirical formula & $\mathrm{C}_{30} \mathrm{H}_{27} \mathrm{NO}_{3}$ \\
\hline Formula weight & 449.52 \\
\hline Temperature/K & $100(2)$ \\
\hline Crystal system & Monoclinic \\
\hline Space group & $\mathrm{P} 2{ }_{1} / \mathrm{n}$ \\
\hline $\mathrm{a} / \AA$ & $16.4257(2)$ \\
\hline $\mathrm{b} / \AA$ & $18.31540(10)$ \\
\hline $\mathrm{c} / \AA$ & $16.5931(2)$ \\
\hline$\alpha /^{\circ}$ & 90 \\
\hline$\beta /{ }^{\circ}$ & $111.9520(10)$ \\
\hline$\gamma /{ }^{\circ}$ & 90 \\
\hline Volume $/ \AA^{3}$ & $4629.99(9)$ \\
\hline $\mathrm{Z}$ & 8 \\
\hline$\rho_{\text {calc }} \mathrm{g} / \mathrm{cm}^{3}$ & 1.290 \\
\hline$\mu / \mathrm{mm}^{-1}$ & 0.657 \\
\hline $\mathrm{F}()$ & 1904.0 \\
\hline Crystal size $/ \mathrm{mm}^{3}$ & $0.35 \times 0.32 \times 0.15$ \\
\hline Radiation & $\operatorname{CuK} \alpha(\lambda=1.54184)$ \\
\hline $2 \Theta$ range for data collection ${ }^{\circ}$ & 6.46 to 144.624 \\
\hline Index ranges & $-20 \leq \mathrm{h} \leq 20,-18 \leq \mathrm{k} \leq 22,-20 \leq 1 \leq 20$ \\
\hline Reflections collected & 52607 \\
\hline Independent reflections & $9037\left[\mathrm{R}_{\text {int }}=0.0318, \mathrm{R}_{\text {sigma }}=0.0192\right]$ \\
\hline Data/restraints/parameters & $9037 / 0 / 655$ \\
\hline Goodness-of-fit on $\mathrm{F}^{2}$ & 1.031 \\
\hline Final $\mathrm{R}$ indexes $[\mathrm{I}>=2 \sigma(\mathrm{I})]$ & $\mathrm{R}_{1}=0.0416, \mathrm{wR}_{2}=0.1050$ \\
\hline Final $\mathrm{R}$ indexes [all data] & $\mathrm{R}_{1}=0.0441, \mathrm{wR}_{2}=0.1071$ \\
\hline Largest diff. peak/hole / e $\AA^{-3}$ & $0.34 /-0.43$ \\
\hline
\end{tabular}




\section{Calculation details}

Table S5. Energies (a.u.) and cartesian coordinates of stationary points for cyclopropenes 1a-e,j,k,n, 15a, 2a-g, 3a-c, DHPO (6), cycloadducts 11a-e, 12a and the transition states TS1,5-endo, TS-1,4-endo, TS-1,5-exo, TS-1,4-exo corresponding to cycloadducts 11a-e, 12a (M11/cc-pVDZ, PCM = THF).

\begin{tabular}{|c|c|c|c|c|c|c|c|}
\hline \multirow{2}{*}{\multicolumn{4}{|c|}{$\mathrm{E}_{0}=-706.640556{ }^{\text {DHPO (6) }}$}} & \multicolumn{4}{|c|}{ Parent cyclopropene (3a) } \\
\hline & & & & $\mathrm{E}_{0}=$ & 16.493751 & & \\
\hline \multicolumn{4}{|c|}{$E(298 K)=-706.627945$} & \multirow{4}{*}{\multicolumn{4}{|c|}{$\begin{array}{l}E(298 K)=-116.490422 \\
H(298 K)=-116.489478 \\
G(298 K)=-116.517054 \\
\text { Imaginary frequencies }=0\end{array}$}} \\
\hline $\mathrm{H}(2 \mathrm{~s}$ & $\mathrm{K})=-706$. & 7001 & & & & & \\
\hline $\mathrm{G}(2 \mathrm{C}$ & $K)=-706$. & 0434 & & & & & \\
\hline Imag & ary frequen & $e s=0$ & & & & & \\
\hline \multicolumn{4}{|c|}{ Cartesian coordinates: } & \multicolumn{4}{|c|}{ Cartesian coordinates: } \\
\hline $\mathrm{C}$ & 4.102099 & -0.718869 & -0.001216 & $\mathrm{C}$ & 0.501210 & -0.648999 & 0.000000 \\
\hline $\mathrm{C}$ & 4.116764 & 0.678099 & -0.014016 & $\mathrm{C}$ & 0.501210 & 0.648999 & 0.000000 \\
\hline $\mathrm{C}$ & 2.919746 & 1.412201 & -0.018918 & $\mathrm{C}$ & -0.862973 & 0.000000 & 0.000000 \\
\hline $\mathrm{C}$ & 2.889482 & -1.426667 & 0.007094 & $\mathrm{H}$ & -1.467727 & 0.000000 & 0.923494 \\
\hline $\mathrm{C}$ & 1.717673 & -0.692021 & 0.001890 & $\mathrm{H}$ & -1.467727 & 0.000000 & -0.923494 \\
\hline $\mathrm{C}$ & 1.732205 & 0.702664 & -0.010626 & $\mathrm{H}$ & 1.049383 & -1.591586 & 0.000000 \\
\hline $\mathrm{H}$ & 5.051747 & -1.267526 & 0.002672 & $\mathrm{H}$ & 1.049383 & 1.591586 & 0.000000 \\
\hline $\mathrm{H}$ & 5.077807 & 1.206633 & -0.020217 & & & & \\
\hline $\mathrm{H}$ & 2.861463 & -2.523261 & 0.017640 & & & & \\
\hline $\mathrm{H}$ & 2.916914 & 2.509148 & -0.028580 & & & & \\
\hline $\mathrm{C}$ & 0.305409 & 1.203339 & -0.010838 & & & & \\
\hline $\mathrm{O}$ & -0.027858 & 2.390222 & -0.015192 & & & & \\
\hline $\mathrm{C}$ & -0.519133 & 0.026258 & -0.003936 & & & & \\
\hline $\mathrm{C}$ & 0.281414 & -1.175375 & 0.009208 & & & & \\
\hline $\mathrm{O}$ & -0.051650 & -2.357786 & 0.025614 & & & & \\
\hline $\mathrm{N}$ & -1.909947 & 0.068912 & 0.010057 & & & & \\
\hline $\mathrm{C}$ & -2.714650 & -1.183169 & 0.080512 & & & & \\
\hline $\mathrm{C}$ & -2.652052 & 1.129854 & 0.021947 & & & & \\
\hline $\mathrm{C}$ & -4.108055 & 0.806606 & 0.105259 & & & & \\
\hline $\mathrm{H}$ & -2.170439 & 2.113576 & 0.010975 & & & & \\
\hline $\mathrm{C}$ & -4.140096 & -0.700993 & -0.206797 & & & & \\
\hline $\mathrm{H}$ & -4.702036 & 1.418350 & -0.594126 & & & & \\
\hline $\mathrm{H}$ & -4.468371 & 1.040398 & 1.126490 & & & & \\
\hline $\mathrm{H}$ & -2.574370 & -1.599895 & 1.092960 & & & & \\
\hline $\mathrm{H}$ & -2.310290 & -1.906245 & -0.640527 & & & & \\
\hline $\mathrm{H}$ & -4.378212 & -0.858011 & -1.272087 & & & & \\
\hline $\mathrm{H}$ & -4.883362 & -1.246589 & 0.393662 & & & & \\
\hline \multicolumn{4}{|c|}{$\begin{array}{l}\text { 3-Methyl-3-phenylcyclopropene }(\mathbf{3 b}) \\
\mathrm{E}_{0}=-386.599509 \\
\mathrm{E}(298 \mathrm{~K})=-386.590775 \\
\mathrm{H}(298 \mathrm{~K})=-386.589831\end{array}$} & \multicolumn{4}{|c|}{$\begin{array}{l}\text { Methyl 1-methylcycloprop-2-ene-1- } \\
\quad \text { carboxylate (3c) } \\
\mathrm{E}_{0}=-383.518236 \\
\mathrm{E}(298 \mathrm{~K})=-383.509567\end{array}$} \\
\hline
\end{tabular}




\begin{tabular}{|c|c|c|c|c|c|c|c|}
\hline \multirow{2}{*}{\multicolumn{4}{|c|}{$G(298 K)=-386.633505$}} & \multirow{2}{*}{\multicolumn{4}{|c|}{$H(298 K)=-383.508623$}} \\
\hline & & & & & & & \\
\hline \multicolumn{4}{|c|}{ Imaginary frequencies $=0$} & \multicolumn{4}{|c|}{$\begin{array}{l}\mathrm{G}(298 \mathrm{~K})=-383.551458 \\
\text { Imaginary frequencies }=0\end{array}$} \\
\hline \multicolumn{4}{|c|}{ Cartesian coordinates: } & & & & \\
\hline $\mathrm{C}$ & -2.282535 & 1.841492 & -0.580094 & \multicolumn{4}{|c|}{ Cartesian coordinates: } \\
\hline $\mathrm{C}$ & -2.213537 & 1.621330 & 0.696959 & $\mathrm{C}$ & -1.173006 & 0.390692 & -0.690528 \\
\hline $\mathrm{C}$ & -0.959122 & 1.291444 & -0.087002 & $\mathrm{C}$ & -1.094012 & 0.186116 & 0.584038 \\
\hline $\mathrm{H}$ & -2.833137 & 2.191837 & -1.455146 & C & -0.164354 & 1.252201 & 0.041327 \\
\hline $\mathrm{H}$ & -2.663360 & 1.649294 & 1.691203 & $\mathrm{H}$ & -1.599159 & 0.177523 & -1.671144 \\
\hline $\mathrm{C}$ & 0.215036 & 2.241292 & 0.013259 & $\mathrm{H}$ & -1.402645 & -0.330474 & 1.493170 \\
\hline $\mathrm{C}$ & -0.610491 & -0.165214 & -0.357169 & $\mathrm{C}$ & -0.401419 & 2.732000 & 0.293582 \\
\hline $\mathrm{H}$ & -1.533639 & -0.765992 & -0.411226 & $\mathrm{H}$ & -1.483031 & 2.923976 & 0.391917 \\
\hline $\mathrm{H}$ & 0.026836 & -0.585355 & 0.443692 & $\mathrm{H}$ & -0.007386 & 3.345202 & -0.536323 \\
\hline $\mathrm{H}$ & -0.067832 & -0.281617 & -1.314299 & $\mathrm{H}$ & 0.102196 & 3.063505 & 1.219053 \\
\hline $\mathrm{C}$ & 0.009262 & 3.607933 & 0.259635 & $\mathrm{C}$ & 1.272172 & 0.823862 & -0.116565 \\
\hline $\mathrm{C}$ & 1.080669 & 4.493448 & 0.354247 & $\mathrm{O}$ & 1.639808 & -0.313490 & -0.322358 \\
\hline $\mathrm{C}$ & 2.391085 & 4.033695 & 0.204419 & $\mathrm{O}$ & 2.128835 & 1.851377 & -0.004079 \\
\hline $\mathrm{C}$ & 2.611560 & 2.679443 & -0.040524 & $\mathrm{C}$ & 3.511510 & 1.507918 & -0.144987 \\
\hline $\mathrm{C}$ & 1.535449 & 1.793709 & -0.134921 & $\mathrm{H}$ & 3.694988 & 1.064055 & -1.138905 \\
\hline $\mathrm{H}$ & 1.737858 & 0.733990 & -0.328127 & $\mathrm{H}$ & 3.804704 & 0.779544 & 0.630948 \\
\hline $\mathrm{H}$ & -1.014316 & 3.984342 & 0.379677 & $\mathrm{H}$ & 4.074256 & 2.445472 & -0.029146 \\
\hline $\mathrm{H}$ & 0.890365 & 5.556767 & 0.547535 & & & & \\
\hline $\mathrm{H}$ & 3.235634 & 4.729489 & 0.278562 & & & & \\
\hline $\mathrm{H}$ & 3.634637 & 2.301730 & -0.160678 & & & & \\
\hline \multicolumn{4}{|c|}{$\begin{array}{l}E(298 K)=-155.756696 \\
H(298 K)=-155.755751 \\
G(298 K)=-155.788274 \\
\text { Imaginary frequencies }=0\end{array}$} & \multicolumn{4}{|c|}{$\begin{array}{l}E(298 \mathrm{~K})=-347.335439 \\
H(298 \mathrm{~K})=-347.334494 \\
G(298 \mathrm{~K})=-347.375510 \\
\text { Imaginary frequencies }=0\end{array}$} \\
\hline \multicolumn{4}{|c|}{ Cartesian coordinates: } & \multicolumn{4}{|c|}{ Cartesian coordinates: } \\
\hline $\mathrm{C}$ & -0.156769 & 0.087005 & 0.000192 & $\mathrm{C}$ & -1.620316 & 0.125070 & -0.000001 \\
\hline $\mathrm{C}$ & 0.918833 & 0.816648 & 0.000107 & $\mathrm{C}$ & -2.740715 & 0.790014 & -0.000025 \\
\hline $\mathrm{C}$ & 1.146246 & -0.677374 & -0.000041 & $\mathrm{C}$ & -2.870844 & -0.715631 & 0.000022 \\
\hline $\mathrm{H}$ & 1.483393 & -1.182155 & -0.923969 & $\mathrm{H}$ & -3.279920 & 1.737766 & -0.000060 \\
\hline $\mathrm{H}$ & 1.483939 & -1.182395 & 0.923544 & $\mathrm{H}$ & -3.176212 & -1.237099 & 0.924594 \\
\hline $\mathrm{H}$ & 1.396274 & 1.797354 & -0.000570 & $\mathrm{H}$ & -3.176209 & -1.237157 & -0.924518 \\
\hline $\mathrm{C}$ & -1.629218 & -0.069384 & -0.000037 & $\mathrm{C}$ & -0.166144 & 0.064234 & 0.000000 \\
\hline $\mathrm{H}$ & -1.950204 & -0.640747 & 0.889292 & $\mathrm{C}$ & 0.475127 & -1.180868 & -0.000007 \\
\hline $\mathrm{H}$ & -1.949764 & -0.641955 & -0.888756 & $\mathrm{C}$ & 1.867581 & -1.252941 & -0.000009 \\
\hline $\mathrm{H}$ & -2.138190 & 0.908528 & -0.000873 & $\mathrm{C}$ & 2.626891 & -0.081933 & -0.000001 \\
\hline & & & & $\mathrm{C}$ & 1.992122 & 1.163723 & 0.000008 \\
\hline
\end{tabular}




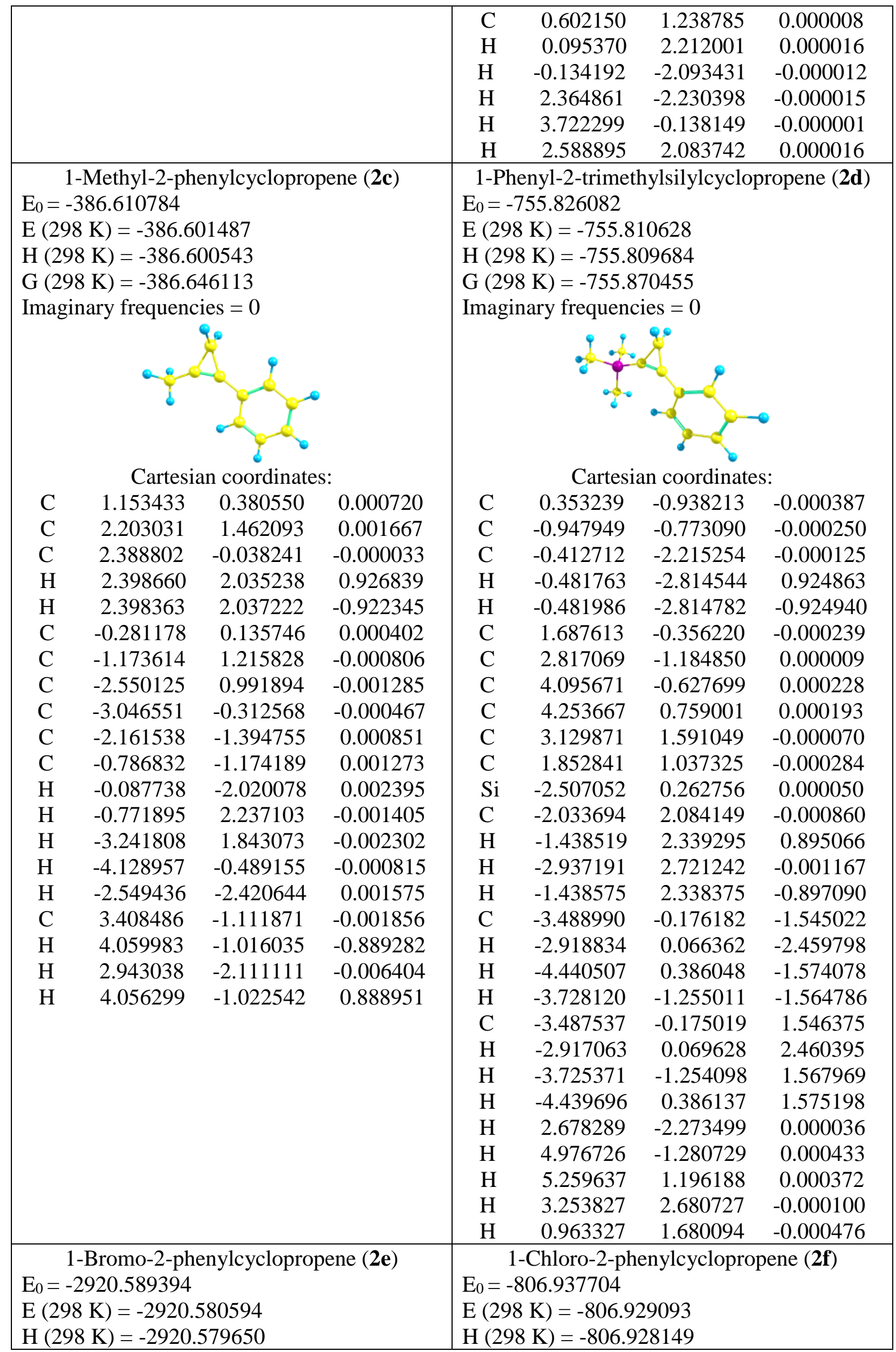




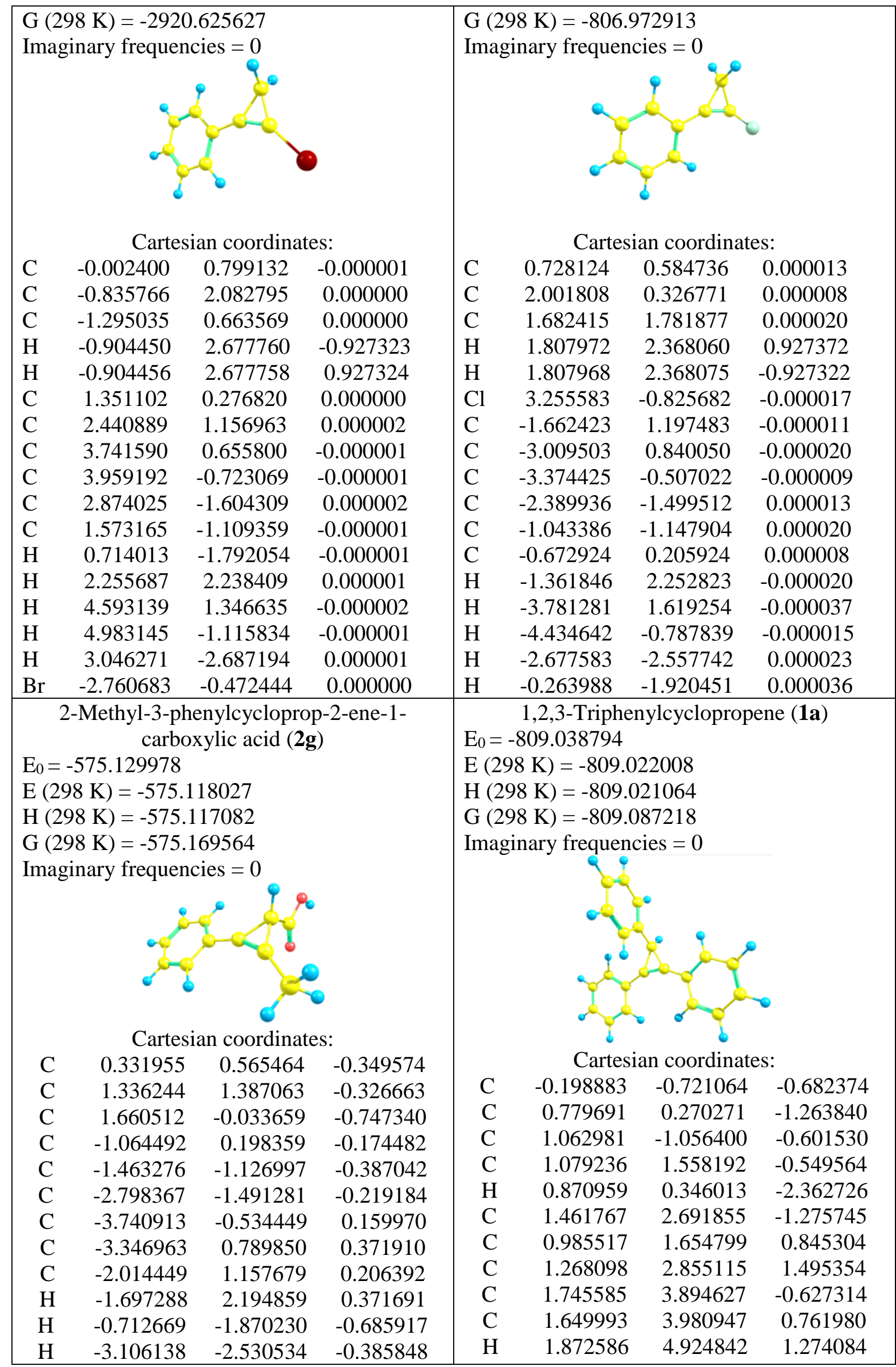




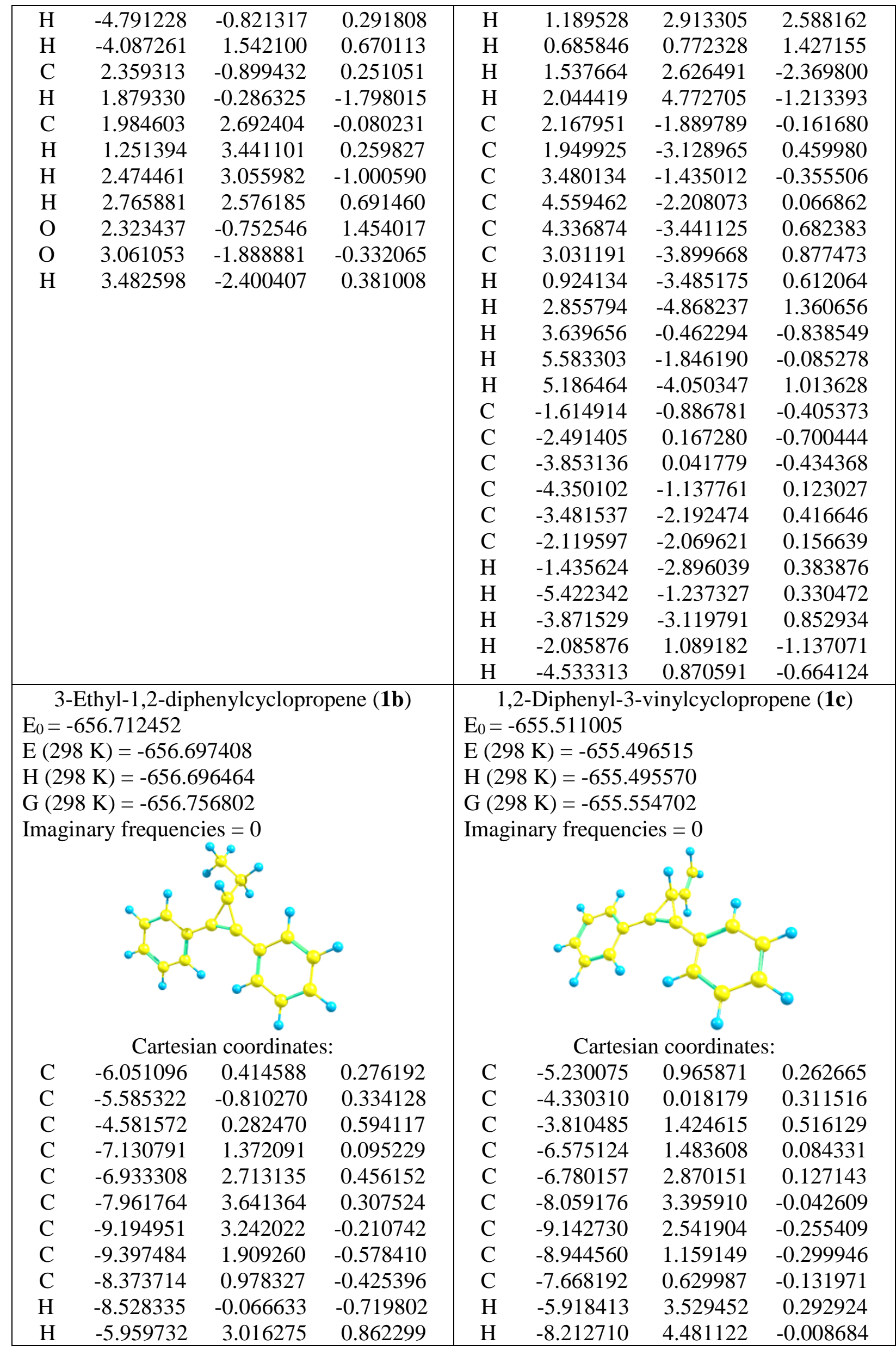




\begin{tabular}{|c|c|c|c|c|c|c|c|}
\hline $\mathrm{H}$ & -7.799543 & 4.686700 & 0.596568 & $\mathrm{H}$ & -10.149683 & 2.955431 & -0.388512 \\
\hline $\mathrm{H}$ & -10.003157 & 3.973439 & -0.331187 & $\mathrm{H}$ & -9.795094 & 0.487752 & -0.467935 \\
\hline $\mathrm{H}$ & -10.363924 & 1.593549 & -0.989377 & $\mathrm{H}$ & -7.509416 & -0.454348 & -0.167408 \\
\hline $\mathrm{C}$ & -5.726760 & -2.254467 & 0.261715 & $\mathrm{C}$ & -3.875539 & -1.358820 & 0.233774 \\
\hline $\mathrm{C}$ & -6.974239 & -2.861827 & 0.046847 & $\mathrm{C}$ & -4.776685 & -2.422385 & 0.069789 \\
\hline $\mathrm{C}$ & -7.076906 & -4.248658 & -0.014413 & $\mathrm{C}$ & -4.306629 & -3.730519 & -0.002949 \\
\hline $\mathrm{C}$ & -5.938046 & -5.044528 & 0.136368 & $\mathrm{C}$ & -2.936234 & -3.990127 & 0.085383 \\
\hline $\mathrm{C}$ & -4.694600 & -4.447841 & 0.352024 & $\mathrm{C}$ & -2.035246 & -2.936375 & 0.248244 \\
\hline $\mathrm{C}$ & -4.588878 & -3.060001 & 0.415665 & $\mathrm{C}$ & -2.501798 & -1.625783 & 0.323605 \\
\hline $\mathrm{H}$ & -3.617039 & -2.578828 & 0.585131 & $\mathrm{H}$ & -1.805066 & -0.787091 & 0.449952 \\
\hline $\mathrm{H}$ & -7.867828 & -2.236191 & -0.064949 & $\mathrm{H}$ & -5.851470 & -2.215902 & 0.002791 \\
\hline $\mathrm{H}$ & -8.055014 & -4.715790 & -0.180680 & $\mathrm{H}$ & -5.015129 & -4.557756 & -0.129787 \\
\hline $\mathrm{H}$ & -6.021958 & -6.136837 & 0.086420 & $\mathrm{H}$ & -2.569224 & -5.021909 & 0.027005 \\
\hline $\mathrm{H}$ & -3.799628 & -5.070078 & 0.472037 & $\mathrm{H}$ & -0.959680 & -3.138277 & 0.317519 \\
\hline $\mathrm{C}$ & -3.513229 & 0.644898 & -0.431028 & $\mathrm{C}$ & -3.036263 & 2.103951 & -0.561210 \\
\hline $\mathrm{H}$ & -4.263911 & 0.455734 & 1.642268 & $\mathrm{H}$ & -3.531368 & 1.741162 & 1.538943 \\
\hline $\mathrm{C}$ & -3.237610 & 2.148737 & -0.477833 & $\mathrm{C}$ & -2.082841 & 3.014409 & -0.345494 \\
\hline $\mathrm{H}$ & -3.841117 & 0.288983 & -1.426952 & $\mathrm{H}$ & -3.294636 & 1.809902 & -1.591827 \\
\hline $\mathrm{H}$ & -2.577701 & 0.101467 & -0.191119 & $\mathrm{H}$ & -1.812651 & 3.319122 & 0.675848 \\
\hline $\mathrm{H}$ & -4.132525 & 2.698630 & -0.823504 & $\mathrm{H}$ & -1.540500 & 3.486354 & -1.173824 \\
\hline $\mathrm{H}$ & -2.404716 & 2.392676 & -1.161113 & & & & \\
\hline $\mathrm{H}$ & -2.974237 & 2.531405 & 0.526537 & & & & \\
\hline \multirow{7}{*}{\multicolumn{4}{|c|}{$\begin{array}{l}\text { 1,2-Diphenyl-3-(phenylethynyl)cyc } \\
\mathrm{E}_{0}=-885.134900 \\
\mathrm{E}(298 \mathrm{~K})=-885.116102 \\
\mathrm{H}(298 \mathrm{~K})=-885.115158 \\
\mathrm{G}(298 \mathrm{~K})=-885.186969 \\
\text { Imaginary frequencies }=0 \\
\text { Cartesian coordinates: }\end{array}$}} & \multicolumn{4}{|c|}{$\begin{array}{c}\text { Methyl 2,3-diphenylcycloprop-2-ene-1- } \\
\text { carboxylate (1e) }\end{array}$} \\
\hline & & & & \multicolumn{4}{|c|}{$E_{0}=-805.955$} \\
\hline & & & & \\
\hline & & & & \multicolumn{4}{|c|}{$H(298 K)=-805.937525$} \\
\hline & & & & \multicolumn{4}{|c|}{$\mathrm{G}(298 \mathrm{~K})=-805.002974$} \\
\hline & & & & \multicolumn{4}{|c|}{ Imaginary frequencies $=0$} \\
\hline & & & & \multicolumn{4}{|c|}{ Cartesian coordinates: } \\
\hline $\mathrm{C}$ & -4.657849 & 1.240480 & -1.022050 & $\mathrm{C}$ & -4.679169 & 0.167128 & -0.585694 \\
\hline $\mathrm{C}$ & -4.062208 & 0.080008 & -1.088988 & $\mathrm{C}$ & -4.389036 & -1.096858 & -0.717446 \\
\hline $\mathrm{C}$ & -3.386340 & 1.205491 & -1.842452 & $\mathrm{C}$ & -3.390007 & -0.133192 & -1.314288 \\
\hline $\mathrm{C}$ & -5.681389 & 2.166482 & -0.573173 & $\mathrm{C}$ & -5.479320 & 1.293716 & -0.142077 \\
\hline $\mathrm{C}$ & -5.560658 & 3.525077 & -0.896799 & $\mathrm{C}$ & -5.023284 & 2.596076 & -0.387386 \\
\hline $\mathrm{C}$ & -6.527828 & 4.433474 & -0.472422 & $\mathrm{C}$ & -5.773900 & 3.691277 & 0.034517 \\
\hline $\mathrm{C}$ & -7.621752 & 3.991524 & 0.273671 & $\mathrm{C}$ & -6.983322 & 3.493144 & 0.702525 \\
\hline $\mathrm{C}$ & -7.747024 & 2.637865 & 0.597596 & $\mathrm{C}$ & -7.442155 & 2.196373 & 0.949379 \\
\hline $\mathrm{C}$ & -6.781747 & 1.726971 & 0.178704 & $\mathrm{C}$ & -6.695513 & 1.099314 & 0.530368 \\
\hline $\mathrm{H}$ & -6.876451 & 0.664049 & 0.430439 & $\mathrm{H}$ & -7.051914 & 0.080535 & 0.722725 \\
\hline $\mathrm{H}$ & -4.694350 & 3.859007 & -1.481986 & $\mathrm{H}$ & -4.071100 & 2.738575 & -0.914216 \\
\hline $\mathrm{H}$ & -6.428002 & 5.495513 & -0.725971 & $\mathrm{H}$ & -5.412536 & 4.708112 & -0.159418 \\
\hline
\end{tabular}




\begin{tabular}{|c|c|c|c|c|c|c|c|}
\hline $\mathrm{H}$ & -8.383617 & 4.706858 & 0.606062 & $\mathrm{H}$ & -7.574209 & 4.355320 & 1.034289 \\
\hline $\mathrm{H}$ & -8.606393 & 2.290304 & 1.183138 & $\mathrm{H}$ & -8.392147 & 2.040483 & 1.474126 \\
\hline $\mathrm{C}$ & -3.887218 & -1.326143 & -0.774752 & $\mathrm{C}$ & -4.602899 & -2.521320 & -0.540124 \\
\hline $\mathrm{C}$ & -4.840106 & -2.028857 & -0.021327 & $\mathrm{C}$ & -5.758954 & -3.008986 & 0.088272 \\
\hline $\mathrm{C}$ & -4.644215 & -3.376510 & 0.265759 & $\mathrm{C}$ & -5.937376 & -4.379566 & 0.250547 \\
\hline $\mathrm{C}$ & -3.498407 & -4.032282 & -0.192580 & $\mathrm{C}$ & -4.966747 & -5.273064 & -0.210469 \\
\hline $\mathrm{C}$ & -2.546851 & -3.336694 & -0.940406 & $\mathrm{C}$ & -3.815266 & -4.792503 & -0.836064 \\
\hline $\mathrm{H}$ & -3.346896 & -5.094069 & 0.036003 & $\mathrm{C}$ & -3.632727 & -3.421342 & -1.001661 \\
\hline $\mathrm{C}$ & -2.739566 & -1.988256 & -1.232461 & $\mathrm{H}$ & -2.732236 & -3.031121 & -1.492954 \\
\hline $\mathrm{H}$ & -1.646661 & -3.849819 & -1.298940 & $\mathrm{H}$ & -5.110406 & -6.352472 & -0.080693 \\
\hline $\mathrm{H}$ & -5.737743 & -1.510956 & 0.336744 & $\mathrm{H}$ & -3.052985 & -5.492453 & -1.198190 \\
\hline $\mathrm{H}$ & -5.391878 & -3.922570 & 0.853138 & $\mathrm{H}$ & -6.518489 & -2.305053 & 0.448232 \\
\hline $\mathrm{H}$ & -1.998328 & -1.428335 & -1.816840 & $\mathrm{H}$ & -6.842280 & -4.756629 & 0.741577 \\
\hline $\mathrm{C}$ & -2.160825 & 1.805562 & -1.322694 & $\mathrm{C}$ & -2.076045 & 0.096990 & -0.632557 \\
\hline $\mathrm{H}$ & -3.459217 & 1.231528 & -2.943364 & $\mathrm{H}$ & -3.313684 & -0.002122 & -2.406370 \\
\hline $\mathrm{C}$ & -1.138282 & 2.309461 & -0.896584 & $\mathrm{O}$ & -1.047241 & 0.391151 & -1.204141 \\
\hline $\mathrm{C}$ & 0.071831 & 2.903301 & -0.383242 & $\mathrm{O}$ & -2.150422 & -0.057397 & 0.698624 \\
\hline $\mathrm{C}$ & 0.288984 & 2.984561 & 1.001556 & $\mathrm{C}$ & -0.921400 & 0.152894 & 1.400377 \\
\hline $\mathrm{C}$ & 1.459204 & 3.557601 & 1.494000 & $\mathrm{H}$ & -0.551677 & 1.178115 & 1.225543 \\
\hline $\mathrm{C}$ & 2.423203 & 4.054841 & 0.615071 & $\mathrm{H}$ & -0.156935 & -0.565503 & 1.056882 \\
\hline $\mathrm{C}$ & 2.212085 & 3.976899 & -0.762774 & $\mathrm{H}$ & -1.145633 & -0.001217 & 2.465767 \\
\hline $\mathrm{C}$ & 1.044425 & 3.405115 & -1.262716 & & & & \\
\hline $\mathrm{H}$ & 2.966076 & 4.366060 & -1.457433 & & & & \\
\hline $\mathrm{H}$ & 0.874291 & 3.341293 & -2.343814 & & & & \\
\hline $\mathrm{H}$ & -0.471206 & 2.592507 & 1.687186 & & & & \\
\hline $\mathrm{H}$ & 1.620021 & 3.616491 & 2.577129 & & & & \\
\hline $\mathrm{H}$ & 3.343498 & 4.505204 & 1.005785 & & & & \\
\hline $\begin{array}{l}2,3 \\
\mathrm{E}_{0}= \\
\mathrm{E}(2 \\
\mathrm{H}(2 \\
\mathrm{G}(2 \\
\mathrm{Ima}\end{array}$ & $\begin{array}{l}\text { iphenylcycl } \\
70.407466 \\
\mathrm{~K})=-670.3 \\
\mathrm{~K})=-670.3 \\
\mathrm{~K})=-670 . \\
\text { ary frequen }\end{array}$ & $\begin{array}{l}\text { (1j) } \\
3655 \\
2710 \\
0322 \\
\text { es }=0\end{array}$ & carbonitrile & \multicolumn{4}{|c|}{$\begin{array}{l}E_{0}=-578.192681 \\
E(298 \mathrm{~K})=-578.180589 \\
H(298 \mathrm{~K})=-578.179644 \\
\mathrm{G}(298 \mathrm{~K})=-578.233052 \\
\text { Imaginary frequencies }=0\end{array}$} \\
\hline \multirow{2}{*}{\multicolumn{4}{|c|}{ Cartesian coordinates: }} & \multicolumn{4}{|c|}{ Cartesian coordinates: } \\
\hline & & & & $\mathrm{C}$ & -3.049442 & 0.584125 & -0.016217 \\
\hline $\mathrm{C}$ & -6.118600 & 0.489565 & 0.344580 & $\mathrm{C}$ & -2.218220 & -0.673337 & -0.027454 \\
\hline $\mathrm{C}$ & -5.607997 & -0.710920 & 0.387263 & $\mathrm{C}$ & -1.751623 & 0.759887 & -0.016210 \\
\hline $\mathrm{C}$ & -4.661574 & 0.413641 & 0.736864 & $\mathrm{H}$ & -2.138231 & -1.263367 & -0.958205 \\
\hline $\mathrm{C}$ & -7.170389 & 1.467589 & 0.138385 & $\mathrm{H}$ & -2.136174 & -1.278501 & 0.893345 \\
\hline $\mathrm{C}$ & -6.876041 & 2.829968 & 0.283170 & $\mathrm{C}$ & -0.552859 & 1.579633 & -0.010909 \\
\hline $\mathrm{C}$ & -7.872846 & 3.782982 & 0.087167 & $\mathrm{C}$ & -0.622934 & 2.981855 & 0.002162 \\
\hline $\mathrm{C}$ & -9.165605 & 3.381266 & -0.252671 & $\mathrm{C}$ & 0.543404 & 3.741228 & 0.007071 \\
\hline $\mathrm{C}$ & -9.462527 & 2.023464 & -0.398203 & $\mathrm{C}$ & 1.791174 & 3.111718 & -0.001111 \\
\hline $\mathrm{C}$ & -8.470614 & 1.067101 & -0.204744 & $\mathrm{C}$ & 1.868474 & 1.718059 & -0.014183 \\
\hline
\end{tabular}




\begin{tabular}{|c|c|c|c|c|c|c|c|}
\hline $\mathrm{H}$ & -8.698685 & 0.000852 & -0.318200 & $\mathrm{C}$ & 0.702671 & 0.954997 & -0.019022 \\
\hline $\mathrm{H}$ & -5.855546 & 3.133373 & 0.549129 & $\mathrm{H}$ & 0.749549 & -0.141263 & -0.029191 \\
\hline $\mathrm{H}$ & -7.639232 & 4.848131 & 0.199923 & $\mathrm{H}$ & -1.602476 & 3.474202 & 0.008586 \\
\hline $\mathrm{H}$ & -9.949870 & 4.132113 & -0.406460 & $\mathrm{H}$ & 0.480751 & 4.835950 & 0.017358 \\
\hline $\mathrm{H}$ & -10.477958 & 1.708477 & -0.665729 & $\mathrm{H}$ & 2.708404 & 3.712837 & 0.002769 \\
\hline $\mathrm{C}$ & -5.629185 & -2.156764 & 0.267086 & $\mathrm{H}$ & 2.846342 & 1.221793 & -0.020634 \\
\hline $\mathrm{C}$ & -6.810279 & -2.835504 & -0.069453 & $\mathrm{C}$ & -4.423640 & 1.053958 & -0.011203 \\
\hline $\mathrm{C}$ & -6.805863 & -4.222595 & -0.178956 & $\mathrm{C}$ & -4.732158 & 2.423696 & 0.000205 \\
\hline $\mathrm{C}$ & -5.627529 & -4.940061 & 0.044004 & $\mathrm{C}$ & -6.059464 & 2.842425 & 0.004430 \\
\hline $\mathrm{C}$ & -4.450613 & -4.267755 & 0.377176 & $\mathrm{C}$ & -7.092822 & 1.901513 & -0.002636 \\
\hline $\mathrm{C}$ & -4.449282 & -2.879312 & 0.489382 & $\mathrm{C}$ & -6.793539 & 0.538162 & -0.013900 \\
\hline $\mathrm{H}$ & -3.530071 & -2.339324 & 0.749519 & $\mathrm{C}$ & -5.465855 & 0.115738 & -0.018168 \\
\hline $\mathrm{H}$ & -7.732259 & -2.268652 & -0.244157 & $\mathrm{H}$ & -5.217133 & -0.952966 & -0.027098 \\
\hline $\mathrm{H}$ & -7.729818 & -4.751032 & -0.441543 & $\mathrm{H}$ & -3.920722 & 3.160923 & 0.005738 \\
\hline $\mathrm{H}$ & -5.627797 & -6.033105 & -0.043931 & $\mathrm{H}$ & -6.292603 & 3.913887 & 0.013350 \\
\hline $\mathrm{H}$ & -3.525682 & -4.830166 & 0.550523 & $\mathrm{H}$ & -8.137636 & 2.234684 & 0.000705 \\
\hline $\mathrm{C}$ & -3.637332 & 0.815737 & -0.227750 & $\mathrm{H}$ & -7.602552 & -0.202119 & -0.019457 \\
\hline $\mathrm{H}$ & -4.350822 & 0.582854 & 1.780951 & & & & \\
\hline $\mathrm{N}$ & -2.825650 & 1.134652 & -0.989870 & & & & \\
\hline \multicolumn{4}{|c|}{$\begin{array}{l}E_{0}=-782.656721 \\
E(298 \mathrm{~K})=-782.642115 \\
H(298 \mathrm{~K})=-782.641171 \\
\mathrm{G}(298 \mathrm{~K})=-782.701592 \\
\text { Imaginary frequencies }=0\end{array}$} & \multicolumn{4}{|c|}{$\begin{array}{l}E_{0}=-692.639421 \\
E(298 \mathrm{~K})=-692.624625 \\
H(298 \mathrm{~K})=-692.623680 \\
G(298 \mathrm{~K})=-692.683882 \\
\text { Imaginary frequencies }=0\end{array}$} \\
\hline \multicolumn{4}{|c|}{ Cartesian coordinates: } & \multicolumn{4}{|c|}{ Cartesian coordinates: } \\
\hline $\mathrm{C}$ & -0.655527 & 0.198126 & -0.356366 & $\mathrm{C}$ & -2.240153 & -0.786732 & 0.273741 \\
\hline $\mathrm{C}$ & -0.000211 & 1.474344 & -0.732156 & $\mathrm{C}$ & -1.830669 & 0.610726 & 0.547790 \\
\hline $\mathrm{C}$ & 0.654677 & 0.197971 & -0.356084 & $\mathrm{C}$ & -0.942839 & -0.559776 & 0.270020 \\
\hline $\mathrm{C}$ & -1.970569 & -0.398180 & -0.201373 & $\mathrm{C}$ & -3.406657 & -1.646580 & 0.149705 \\
\hline $\mathrm{C}$ & -2.110897 & -1.760102 & 0.102098 & $\mathrm{C}$ & -4.679920 & -1.060833 & 0.112366 \\
\hline $\mathrm{C}$ & -3.380596 & -2.310652 & 0.247333 & $\mathrm{C}$ & -5.815544 & -1.859031 & -0.004772 \\
\hline $\mathrm{C}$ & -4.514016 & -1.508262 & 0.092962 & $\mathrm{C}$ & -5.688282 & -3.247407 & -0.080323 \\
\hline $\mathrm{C}$ & -4.377397 & -0.152261 & -0.208729 & $\mathrm{C}$ & -4.422123 & -3.836852 & -0.040233 \\
\hline $\mathrm{C}$ & -3.109020 & 0.403524 & -0.356472 & $\mathrm{C}$ & -3.284561 & -3.041859 & 0.071912 \\
\hline $\mathrm{H}$ & -2.989748 & 1.468481 & -0.592830 & $\mathrm{H}$ & -2.289675 & -3.501671 & 0.111152 \\
\hline $\mathrm{H}$ & -1.218540 & -2.385682 & 0.220555 & $\mathrm{H}$ & -6.582434 & -3.875886 & -0.170892 \\
\hline $\mathrm{H}$ & -3.488774 & -3.375612 & 0.483683 & $\mathrm{H}$ & -4.321898 & -4.927315 & -0.096528 \\
\hline $\mathrm{H}$ & -5.513049 & -1.945071 & 0.209631 & $\mathrm{H}$ & -4.762251 & 0.032184 & 0.166267 \\
\hline $\mathrm{C}$ & 1.969655 & -0.396743 & -0.195253 & $\mathrm{H}$ & -6.808653 & -1.395285 & -0.037296 \\
\hline $\mathrm{C}$ & 2.109090 & -1.735056 & 0.199760 & $\mathrm{C}$ & 0.434427 & -1.014503 & 0.136422 \\
\hline $\mathrm{C}$ & 3.378558 & -2.285330 & 0.347788 & $\mathrm{C}$ & 1.485802 & -0.170227 & 0.520952 \\
\hline $\mathrm{C}$ & 4.512636 & -1.506196 & 0.104367 & $\mathrm{C}$ & 2.806956 & -0.598788 & 0.413094 \\
\hline $\mathrm{C}$ & 4.376864 & -0.172994 & -0.286282 & $\mathrm{C}$ & 3.090148 & -1.871390 & -0.086326 \\
\hline
\end{tabular}




\begin{tabular}{|c|c|c|c|c|c|c|c|}
\hline $\mathrm{C}$ & 3.108668 & 0.383028 & -0.434933 & $\mathrm{C}$ & 2.047960 & -2.715901 & -0.476567 \\
\hline $\mathrm{H}$ & 2.989441 & 1.430737 & -0.738733 & $\mathrm{C}$ & 0.726416 & -2.292043 & -0.364888 \\
\hline $\mathrm{H}$ & 1.216078 & -2.340602 & 0.393390 & $\mathrm{H}$ & -0.094076 & -2.948807 & -0.678181 \\
\hline $\mathrm{H}$ & 3.486174 & -3.331449 & 0.657267 & $\mathrm{H}$ & 1.252778 & 0.828926 & 0.911415 \\
\hline $\mathrm{H}$ & 5.511548 & -1.942883 & 0.222521 & $\mathrm{H}$ & 3.623435 & 0.066019 & 0.719253 \\
\hline $\mathrm{H}$ & 5.266635 & 0.438950 & -0.474773 & $\mathrm{H}$ & 4.130331 & -2.207430 & -0.174488 \\
\hline $\mathrm{H}$ & -5.266714 & 0.477255 & -0.329160 & $\mathrm{H}$ & 2.268555 & -3.714123 & -0.873143 \\
\hline $\mathrm{N}$ & 0.000598 & 2.573920 & 0.297357 & $\mathrm{O}$ & -2.075850 & 1.576881 & -0.450441 \\
\hline $\mathrm{H}$ & 0.000638 & 1.941613 & -1.727591 & $\mathrm{H}$ & -1.895900 & 1.044466 & 1.566494 \\
\hline $\mathrm{O}$ & -0.014663 & 2.262776 & 1.469934 & $\mathrm{C}$ & -1.003433 & 2.487497 & -0.578127 \\
\hline $\mathrm{O}$ & 0.016532 & 3.713176 & -0.126759 & $\mathrm{H}$ & -0.754849 & 2.961540 & 0.396104 \\
\hline & & & & $\mathrm{H}$ & -1.306575 & 3.274745 & -1.288719 \\
\hline & & & & $\mathrm{H}$ & -0.092311 & 1.983800 & -0.965759 \\
\hline $\begin{array}{l}\mathrm{E}_{0}= \\
\mathrm{E}(2 \\
\mathrm{H}(2 \\
\mathrm{G}(2 \\
\mathrm{Ima}\end{array}$ & $\begin{array}{r}\text { Cycloadc } \\
62.483176 \\
\mathrm{~K})=-862.4 \\
\mathrm{~K})=-862 . \\
\mathrm{K})=-862.5 \\
\text { ary frequen }\end{array}$ & $\begin{array}{l}6786 \\
5842 \\
6897\end{array}$ & & $\begin{array}{l}\mathrm{E}_{0}= \\
\mathrm{E}(2 \\
\mathrm{H}(2 \\
\mathrm{G}(2 \\
\mathrm{Ima}\end{array}$ & $\begin{array}{r}\text { Cycload } \\
62.482017 \\
\mathrm{~K})=-862 . \\
\mathrm{K})=-862 . \\
\mathrm{K})=-862 . \\
\text { ary frequen }\end{array}$ & $\begin{array}{l}5496 \\
4551 \\
6246\end{array}$ & \\
\hline & Cartes & n coordinat & & & Cartes & n coordinat & \\
\hline $\mathrm{C}$ & 4.302042 & -0.601577 & -0.695080 & $\mathrm{C}$ & -4.476108 & -0.312687 & -0.879335 \\
\hline $\mathrm{C}$ & 4.360778 & -0.317754 & 0.680186 & $\mathrm{C}$ & -4.590327 & 0.153068 & 0.441799 \\
\hline $\mathrm{C}$ & 3.082921 & -0.638253 & -1.369363 & $\mathrm{C}$ & -3.227688 & -0.513624 & -1.465595 \\
\hline $\mathrm{C}$ & 1.928925 & -0.377369 & -0.633608 & $\mathrm{C}$ & -2.099517 & -0.241812 & -0.694412 \\
\hline $\mathrm{C}$ & 1.986522 & -0.094143 & 0.732441 & $\mathrm{C}$ & -2.213156 & 0.222126 & 0.617551 \\
\hline $\mathrm{C}$ & 3.202255 & -0.063473 & 1.412819 & $\mathrm{C}$ & -3.458451 & 0.429653 & 1.206688 \\
\hline $\mathrm{H}$ & 5.335789 & -0.300372 & 1.181867 & $\mathrm{H}$ & -5.587725 & 0.303842 & 0.871680 \\
\hline $\mathrm{H}$ & 3.233750 & 0.153627 & 2.487164 & $\mathrm{H}$ & -3.532256 & 0.801249 & 2.235590 \\
\hline $\mathrm{H}$ & 5.232395 & -0.838000 & -1.240431 & $\mathrm{H}$ & -5.386893 & -0.517094 & -1.454826 \\
\hline $\mathrm{H}$ & 3.021103 & -0.863924 & -2.440616 & $\mathrm{H}$ & -3.124420 & -0.872310 & -2.496549 \\
\hline $\mathrm{C}$ & 0.510648 & -0.351837 & -1.097286 & $\mathrm{C}$ & -0.658340 & -0.361170 & -1.067105 \\
\hline $\mathrm{C}$ & -0.358419 & 0.151426 & 0.069555 & $\mathrm{C}$ & 0.180286 & -0.071211 & 0.191449 \\
\hline $\mathrm{C}$ & 0.607282 & 0.118531 & 1.267098 & $\mathrm{C}$ & -0.856370 & 0.468002 & 1.192536 \\
\hline $\mathrm{N}$ & -1.594513 & -0.538279 & 0.289473 & $\mathrm{~N}$ & 1.323490 & 0.777989 & 0.020717 \\
\hline $\mathrm{C}$ & -2.623505 & -0.116000 & -0.592090 & $\mathrm{C}$ & 2.454367 & -0.010062 & -0.447395 \\
\hline $\mathrm{C}$ & -2.313543 & 1.484258 & -0.573151 & $\mathrm{C}$ & 2.308940 & -1.310474 & 0.328481 \\
\hline $\mathrm{C}$ & -0.841881 & 1.600551 & -0.215605 & $\mathrm{C}$ & 0.837014 & -1.371439 & 0.699754 \\
\hline $\mathrm{C}$ & -1.790374 & -1.970888 & 0.372086 & $\mathrm{C}$ & 1.369532 & 2.060777 & -0.649997 \\
\hline $\mathrm{C}$ & -3.346068 & -2.080520 & 0.347680 & $\mathrm{C}$ & 2.904936 & 2.325355 & -0.722527 \\
\hline $\mathrm{H}$ & -1.343837 & -2.382569 & 1.295506 & $\mathrm{H}$ & 0.825876 & 2.832192 & -0.074610 \\
\hline $\mathrm{H}$ & -1.349476 & -2.508947 & -0.498193 & $\mathrm{H}$ & 0.934071 & 2.019295 & -1.674834 \\
\hline $\mathrm{C}$ & -3.863303 & -0.651419 & 0.017197 & $\mathrm{C}$ & 3.583522 & 1.001741 & -0.266192 \\
\hline $\mathrm{H}$ & -3.740696 & -2.437400 & 1.313361 & $\mathrm{H}$ & 3.199340 & 3.170077 & -0.077991 \\
\hline $\mathrm{H}$ & -3.663744 & -2.804001 & -0.422729 & $\mathrm{H}$ & 3.197252 & 2.584401 & -1.754515 \\
\hline
\end{tabular}




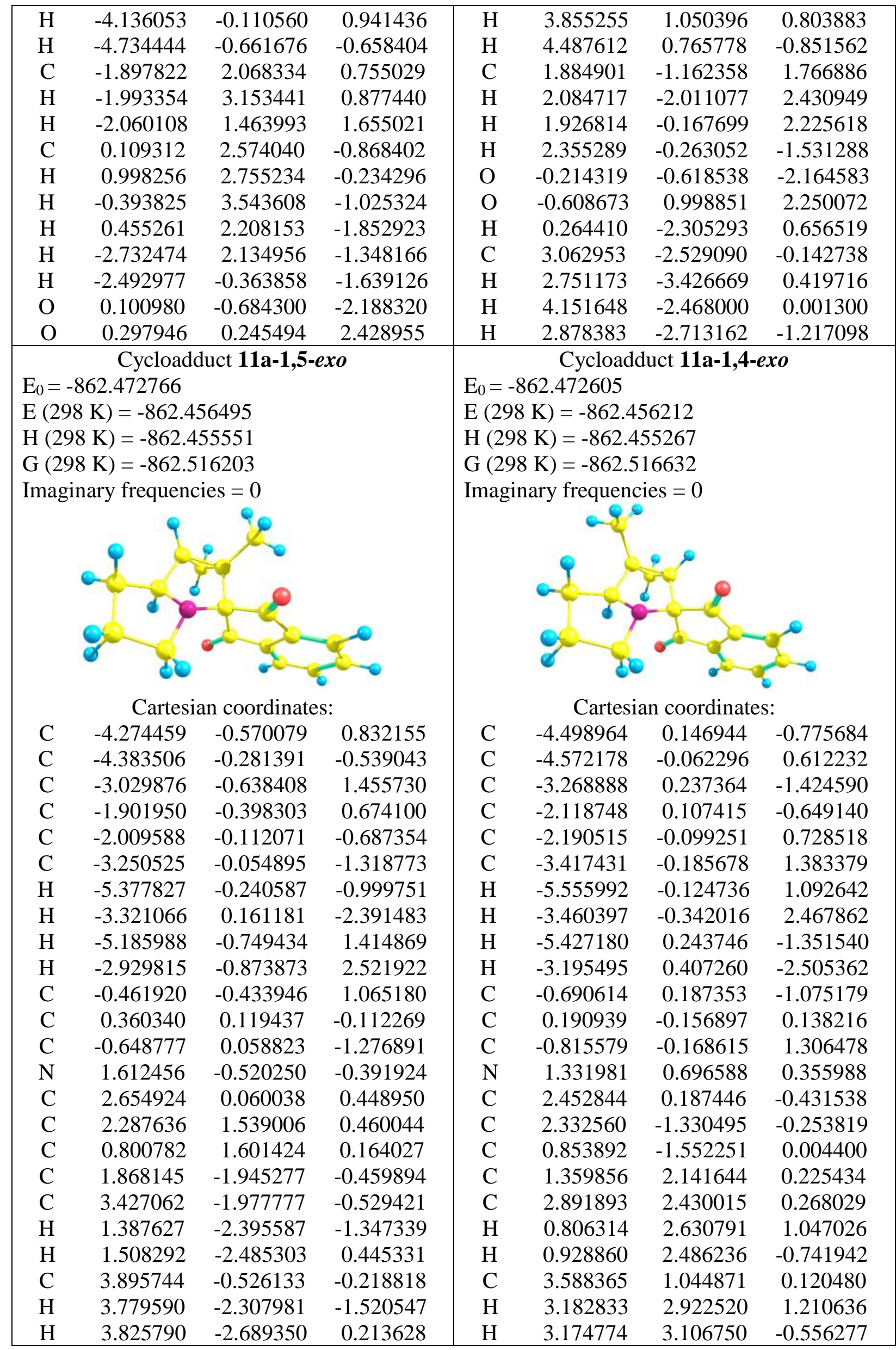




\begin{tabular}{|c|c|c|c|c|c|c|c|}
\hline $\mathrm{H}$ & 4.102431 & 0.025286 & -1.153077 & $\mathrm{H}$ & 3.899767 & 0.661391 & 1.107695 \\
\hline $\mathrm{H}$ & 4.795398 & -0.498678 & 0.417729 & $\mathrm{H}$ & 4.471038 & 1.083602 & -0.539152 \\
\hline $\mathrm{H}$ & 2.592368 & -0.343707 & 1.488637 & $\mathrm{H}$ & 2.317962 & 0.441919 & -1.510719 \\
\hline $\mathrm{O}$ & -0.010678 & -0.856057 & 2.109270 & $\mathrm{O}$ & -0.288109 & 0.505579 & -2.174524 \\
\hline $\mathrm{O}$ & -0.380217 & 0.123873 & -2.453327 & $\mathrm{O}$ & -0.533809 & -0.233275 & 2.479494 \\
\hline $\mathrm{C}$ & 1.315830 & 1.932697 & 1.544984 & $\mathrm{C}$ & 1.472044 & -1.979187 & -1.306826 \\
\hline $\mathrm{H}$ & 1.165317 & 1.214526 & 2.360317 & $\mathrm{C}$ & 3.392305 & -2.096603 & 0.499516 \\
\hline $\mathrm{H}$ & 1.257394 & 2.988544 & 1.835101 & $\mathrm{H}$ & 0.525250 & -2.327259 & 0.707101 \\
\hline $\mathrm{C}$ & 0.203876 & 2.700476 & -0.686627 & $\mathrm{H}$ & 1.239786 & -1.397604 & -2.207614 \\
\hline $\mathrm{H}$ & 2.962979 & 2.280607 & 0.017288 & $\mathrm{H}$ & 1.573304 & -3.060720 & -1.458831 \\
\hline $\mathrm{H}$ & 0.386103 & 2.517756 & -1.759834 & $\mathrm{H}$ & 4.366342 & -2.047249 & -0.021656 \\
\hline $\mathrm{H}$ & 0.657597 & 3.669316 & -0.414858 & $\mathrm{H}$ & 3.525125 & -1.689803 & 1.518178 \\
\hline $\mathrm{H}$ & -0.888654 & 2.784772 & -0.528495 & $\mathrm{H}$ & 3.108505 & -3.160030 & 0.590842 \\
\hline \multicolumn{4}{|c|}{$\begin{array}{l}E_{0}=-1054.064854 \\
E(298 \mathrm{~K})=-1054.045141 \\
H(298 \mathrm{~K})=-1054.044196 \\
G(298 \mathrm{~K})=-1054.113877 \\
\text { Imaginary frequencies }=0\end{array}$} & $\begin{array}{l}\mathrm{E}_{0}= \\
\mathrm{E}(2 \\
\mathrm{H}(2 \\
\mathrm{G}(2 \\
\mathrm{Ima}\end{array}$ & $\begin{array}{r}\text { Cycload } \\
054.063415 \\
\mathrm{~K})=-1054 \\
\mathrm{~K})=-1054 \\
\mathrm{~K})=-1054 \\
\text { ary frequen }\end{array}$ & $\begin{array}{l}\text { ct 11b-1,4-c } \\
43719 \\
42775 \\
13776 \\
\text { es }=0\end{array}$ & ado \\
\hline \multicolumn{4}{|c|}{ Cartesian coordinates: } & \multicolumn{4}{|c|}{ Cartesian coordinates: } \\
\hline $\mathrm{C}$ & 3.365350 & -2.294234 & -0.926503 & $\mathrm{C}$ & -4.853308 & -1.983695 & -0.623202 \\
\hline $\mathrm{C}$ & 3.306030 & -2.640257 & 0.434002 & $\mathrm{C}$ & -5.292542 & -1.213002 & 0.467152 \\
\hline $\mathrm{C}$ & 2.298168 & -1.651013 & -1.551827 & $\mathrm{C}$ & -3.548504 & -1.875152 & -1.100866 \\
\hline $\mathrm{C}$ & 1.179014 & -1.355679 & -0.777361 & $\mathrm{C}$ & -2.697846 & -0.977057 & -0.459046 \\
\hline $\mathrm{C}$ & 1.122164 & -1.694021 & 0.576425 & $\mathrm{C}$ & -3.134022 & -0.211501 & 0.623774 \\
\hline $\mathrm{C}$ & 2.180818 & -2.347250 & 1.202951 & $\mathrm{C}$ & -4.437704 & -0.315724 & 1.104627 \\
\hline $\mathrm{H}$ & 4.161471 & -3.149159 & 0.894030 & $\mathrm{H}$ & -6.326160 & -1.321589 & 0.816754 \\
\hline $\mathrm{H}$ & 2.122630 & -2.611524 & 2.265523 & $\mathrm{H}$ & -4.767864 & 0.294431 & 1.953546 \\
\hline $\mathrm{H}$ & 4.266326 & -2.538387 & -1.501689 & $\mathrm{H}$ & -5.552617 & -2.678790 & -1.103162 \\
\hline $\mathrm{H}$ & 2.329593 & -1.376304 & -2.612915 & $\mathrm{H}$ & -3.192345 & -2.468209 & -1.951640 \\
\hline $\mathrm{C}$ & -0.078290 & -0.657864 & -1.174705 & $\mathrm{C}$ & -1.269314 & -0.669254 & -0.756060 \\
\hline $\mathrm{C}$ & -0.863809 & -0.352792 & 0.114418 & $\mathrm{C}$ & -0.774522 & 0.361531 & 0.276922 \\
\hline $\mathrm{C}$ & -0.173972 & -1.240432 & 1.162769 & $\mathrm{C}$ & -2.035931 & 0.682113 & 1.099229 \\
\hline $\mathrm{N}$ & -2.286533 & -0.451612 & 0.065083 & $\mathrm{~N}$ & -0.158668 & 1.541898 & -0.271727 \\
\hline $\mathrm{C}$ & -2.857142 & 0.778942 & -0.469163 & $\mathrm{C}$ & 1.226871 & 1.251756 & -0.605300 \\
\hline $\mathrm{C}$ & -1.972063 & 1.839801 & 0.159445 & $\mathrm{C}$ & 1.671935 & 0.361316 & 0.550263 \\
\hline $\mathrm{C}$ & -0.661086 & 1.150452 & 0.486242 & $\mathrm{C}$ & 0.380946 & -0.226330 & 1.102101 \\
\hline $\mathrm{C}$ & -3.074320 & -1.562866 & -0.427416 & $\mathrm{C}$ & -0.684058 & 2.394051 & -1.319055 \\
\hline $\mathrm{C}$ & -4.509888 & -0.954035 & -0.442011 & $\mathrm{C}$ & 0.541616 & 3.309385 & -1.621955 \\
\hline $\mathrm{H}$ & -2.985065 & -2.439617 & 0.239712 & $\mathrm{H}$ & -1.569730 & 2.956948 & -0.972532 \\
\hline $\mathrm{H}$ & -2.772047 & -1.873393 & -1.453238 & $\mathrm{H}$ & -0.970519 & 1.817097 & -2.228452 \\
\hline $\mathrm{C}$ & -4.330491 & 0.559144 & -0.132761 & $\mathrm{C}$ & 1.745900 & 2.661430 & -0.877562 \\
\hline $\mathrm{H}$ & -5.163222 & -1.440678 & 0.301116 & $\mathrm{H}$ & 0.365825 & 4.340564 & -1.273496 \\
\hline $\mathrm{H}$ & -4.974368 & -1.101611 & -1.431901 & $\mathrm{H}$ & 0.722963 & 3.356970 & -2.709096 \\
\hline
\end{tabular}




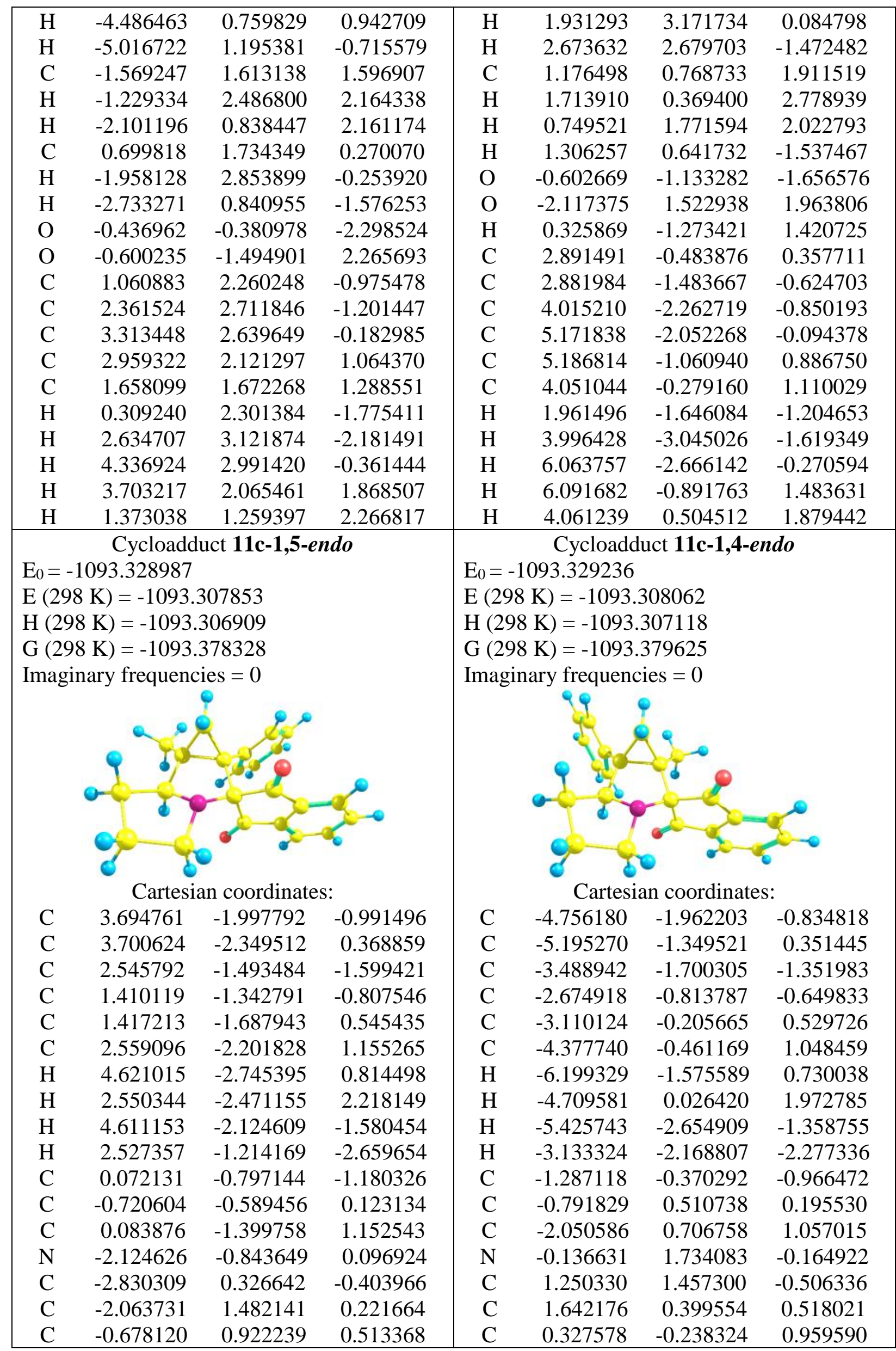




\begin{tabular}{|c|c|c|c|c|c|c|c|}
\hline $\mathrm{C}$ & -2.794904 & -2.021830 & -0.414182 & $\mathrm{C}$ & -0.612680 & 2.748670 & -1.083290 \\
\hline $\mathrm{C}$ & -4.288716 & -1.573694 & -0.389251 & $\mathrm{C}$ & 0.644663 & 3.660084 & -1.223893 \\
\hline $\mathrm{H}$ & -2.598192 & -2.901080 & 0.225952 & $\mathrm{H}$ & -1.488887 & 3.281948 & -0.671989 \\
\hline $\mathrm{H}$ & -2.479335 & -2.269391 & -1.452926 & $\mathrm{H}$ & -0.895874 & 2.322322 & -2.073280 \\
\hline $\mathrm{C}$ & -4.268441 & -0.053767 & -0.059288 & $\mathrm{C}$ & 1.811487 & 2.876243 & -0.555065 \\
\hline $\mathrm{H}$ & -4.866494 & -2.138870 & 0.360802 & $\mathrm{H}$ & 0.488008 & 4.635862 & -0.735271 \\
\hline $\mathrm{H}$ & -4.757105 & -1.759119 & -1.370899 & $\mathrm{H}$ & 0.855601 & 3.857369 & -2.288595 \\
\hline $\mathrm{H}$ & -4.435841 & 0.114145 & 1.020220 & $\mathrm{H}$ & 1.982938 & 3.234061 & 0.476243 \\
\hline $\mathrm{H}$ & -5.024500 & 0.512995 & -0.627537 & $\mathrm{H}$ & 2.755384 & 2.955281 & -1.118908 \\
\hline $\mathrm{C}$ & -1.617988 & 1.257344 & 1.647075 & $\mathrm{C}$ & 1.113849 & 0.632706 & 1.910641 \\
\hline $\mathrm{H}$ & -1.364779 & 2.153225 & 2.227358 & $\mathrm{H}$ & 1.615139 & 0.106223 & 2.731867 \\
\hline $\mathrm{H}$ & -2.048786 & 0.417737 & 2.204609 & $\mathrm{H}$ & 0.710967 & 1.624737 & 2.142997 \\
\hline $\mathrm{C}$ & 0.616634 & 1.643429 & 0.310834 & $\mathrm{H}$ & 1.338458 & 0.991074 & -1.516484 \\
\hline $\mathrm{H}$ & -2.727162 & 0.424892 & -1.512288 & $\mathrm{O}$ & -0.645872 & -0.651323 & -1.957401 \\
\hline $\mathrm{O}$ & -0.334034 & -0.559272 & -2.297064 & $\mathrm{O}$ & -2.154949 & 1.467607 & 1.990811 \\
\hline $\mathrm{O}$ & -0.291962 & -1.714054 & 2.258484 & $\mathrm{C}$ & 2.834010 & -0.458456 & 0.240301 \\
\hline $\mathrm{C}$ & 0.947434 & 2.183704 & -0.938187 & $\mathrm{C}$ & 2.825314 & -1.283805 & -0.894095 \\
\hline $\mathrm{C}$ & 2.199756 & 2.761453 & -1.148275 & $\mathrm{C}$ & 3.918598 & -2.097691 & -1.184209 \\
\hline $\mathrm{C}$ & 3.135468 & 2.798959 & -0.112828 & $\mathrm{C}$ & 5.033376 & -2.099836 & -0.341298 \\
\hline $\mathrm{C}$ & 2.811467 & 2.267474 & 1.137044 & $\mathrm{C}$ & 5.047595 & -1.283776 & 0.789757 \\
\hline $\mathrm{C}$ & 1.556844 & 1.695541 & 1.346508 & $\mathrm{C}$ & 3.951954 & -0.466702 & 1.078522 \\
\hline $\mathrm{H}$ & 0.213329 & 2.133313 & -1.753532 & $\mathrm{H}$ & 1.934884 & -1.288116 & -1.540742 \\
\hline $\mathrm{H}$ & 2.448841 & 3.182335 & -2.130124 & $\mathrm{H}$ & 3.899766 & -2.742075 & -2.071726 \\
\hline $\mathrm{H}$ & 4.122102 & 3.247913 & -0.280081 & $\mathrm{H}$ & 5.893002 & -2.742563 & -0.567210 \\
\hline $\mathrm{H}$ & 3.542594 & 2.297280 & 1.954168 & $\mathrm{H}$ & 5.920084 & -1.281695 & 1.454653 \\
\hline $\mathrm{H}$ & 1.295518 & 1.269882 & 2.325858 & $\mathrm{H}$ & 3.962122 & 0.176873 & 1.967911 \\
\hline $\mathrm{C}$ & -2.278178 & 2.876946 & -0.311223 & $\mathrm{C}$ & 0.179789 & -1.718858 & 1.210666 \\
\hline $\mathrm{H}$ & -2.201402 & 2.896295 & -1.414454 & $\mathrm{H}$ & 1.074197 & -2.102802 & 1.731640 \\
\hline $\mathrm{H}$ & -3.281475 & 3.248878 & -0.033314 & $\mathrm{H}$ & 0.074920 & -2.283028 & 0.264271 \\
\hline $\mathrm{H}$ & -1.528181 & 3.575818 & 0.099385 & $\mathrm{H}$ & -0.701731 & -1.931406 & 1.844785 \\
\hline $\begin{array}{l}E_{0}= \\
E(2 \\
H(2 \\
\text { G (2 } \\
\text { Ima }\end{array}$ & $\begin{array}{r}\text { Cycloadc } \\
462.540905 \\
\mathrm{~K})=-1462 \\
\mathrm{~K})=-1462 \\
\mathrm{~K})=-1462 \\
\text { ary frequen }\end{array}$ & $\begin{array}{l}\text { ct 11d-1,5-c- } \\
13192 \\
12248 \\
99230 \\
\text { es }=0\end{array}$ & ado & $\begin{array}{l}\mathrm{E}_{0} \\
\mathrm{E}( \\
\mathrm{H} \\
\mathrm{G}( \\
\mathrm{Im} a\end{array}$ & $\begin{array}{r}\text { Cycload } \\
1462.542870 \\
3 \mathrm{~K})=-1462 \\
8 \mathrm{~K})=-1462 \\
8 \mathrm{~K})=-1462 \\
\text { nary frequen }\end{array}$ & $\begin{array}{l}515683 \\
514739 \\
598953 \\
\text { ies }=0\end{array}$ & ndo \\
\hline \multicolumn{4}{|c|}{ Cartesian coordinates: } & \multicolumn{4}{|c|}{ Cartesian coordinates: } \\
\hline $\mathrm{C}$ & -4.890136 & -0.092247 & 1.134716 & $\mathrm{C}$ & 4.876359 & -1.086730 & 1.528736 \\
\hline $\mathrm{C}$ & -5.128570 & -0.342357 & -0.227437 & $\mathrm{C}$ & 5.296980 & -0.903883 & 0.199725 \\
\hline $\mathrm{C}$ & -3.617517 & -0.245586 & 1.681791 & $\mathrm{C}$ & 3.596133 & -0.715929 & 1.934577 \\
\hline $\mathrm{C}$ & -2.592197 & -0.647470 & 0.828919 & $\mathrm{C}$ & 2.749617 & -0.160783 & 0.976260 \\
\hline $\mathrm{C}$ & -2.828333 & -0.894140 & -0.524753 & $\mathrm{C}$ & 3.166932 & 0.019611 & -0.343056 \\
\hline $\mathrm{C}$ & -4.099848 & -0.749835 & -1.074963 & $\mathrm{C}$ & 4.446990 & -0.347354 & -0.753919 \\
\hline $\mathrm{H}$ & -6.142674 & -0.215468 & -0.624687 & $\mathrm{H}$ & 6.311854 & -1.205329 & -0.085764 \\
\hline
\end{tabular}




\begin{tabular}{|c|c|c|c|c|c|c|c|}
\hline $\mathrm{H}$ & -4.272909 & -0.952921 & -2.138353 & $\mathrm{H}$ & 4.761513 & -0.198661 & -1.793686 \\
\hline $\mathrm{H}$ & -5.722434 & 0.226224 & 1.773622 & $\mathrm{H}$ & 5.570916 & -1.527950 & 2.253423 \\
\hline $\mathrm{H}$ & -3.417402 & -0.057261 & 2.743205 & $\mathrm{H}$ & 3.253328 & -0.852837 & 2.967254 \\
\hline $\mathrm{C}$ & -1.153755 & -0.883803 & 1.141271 & $\mathrm{C}$ & 1.337898 & 0.296403 & 1.130292 \\
\hline $\mathrm{C}$ & -0.415487 & -1.114360 & -0.189821 & $\mathrm{C}$ & 0.828554 & 0.756855 & -0.248250 \\
\hline $\mathrm{C}$ & -1.564841 & -1.325573 & -1.192598 & $\mathrm{C}$ & 2.069108 & 0.630433 & -1.151381 \\
\hline $\mathrm{N}$ & 0.592153 & -2.127283 & -0.200262 & $\mathrm{~N}$ & 0.265082 & 2.081736 & -0.261890 \\
\hline $\mathrm{C}$ & 1.847023 & -1.567739 & 0.285891 & $\mathrm{C}$ & -1.114416 & 2.011719 & 0.188218 \\
\hline $\mathrm{C}$ & 1.873628 & -0.175158 & -0.339966 & $\mathrm{C}$ & -1.615529 & 0.732167 & -0.470085 \\
\hline $\mathrm{C}$ & 0.402545 & 0.152786 & -0.595732 & $\mathrm{C}$ & -0.362138 & -0.000120 & -0.715065 \\
\hline $\mathrm{C}$ & 0.481865 & -3.474989 & 0.318116 & $\mathrm{C}$ & 0.841091 & 3.281967 & 0.311447 \\
\hline $\mathrm{C}$ & 1.959040 & -3.965998 & 0.237075 & $\mathrm{C}$ & -0.351745 & 4.281324 & 0.209508 \\
\hline $\mathrm{H}$ & -0.208096 & -4.083491 & -0.294101 & $\mathrm{H}$ & 1.731083 & 3.611252 & -0.254667 \\
\hline $\mathrm{H}$ & 0.122779 & -3.493315 & 1.372067 & $\mathrm{H}$ & 1.137098 & 3.140813 & 1.376620 \\
\hline $\mathrm{C}$ & 2.809867 & -2.699333 & -0.070037 & $\mathrm{C}$ & -1.592523 & 3.418416 & -0.164522 \\
\hline $\mathrm{H}$ & 2.084318 & -4.729356 & -0.548547 & $\mathrm{H}$ & -0.160746 & 5.055691 & -0.551679 \\
\hline $\mathrm{H}$ & 2.261014 & -4.427466 & 1.192514 & $\mathrm{H}$ & -0.499765 & 4.798165 & 1.172808 \\
\hline $\mathrm{H}$ & 3.055328 & -2.640146 & -1.146396 & $\mathrm{H}$ & -1.793249 & 3.471833 & -1.249703 \\
\hline $\mathrm{H}$ & 3.748945 & -2.673514 & 0.507897 & $\mathrm{H}$ & -2.501811 & 3.721984 & 0.379586 \\
\hline $\mathrm{C}$ & 1.318745 & -0.118977 & -1.755696 & $\mathrm{C}$ & -1.132825 & 0.513758 & -1.871048 \\
\hline $\mathrm{H}$ & 1.600919 & 0.746405 & -2.369382 & $\mathrm{H}$ & -1.710503 & -0.167834 & -2.507903 \\
\hline $\mathrm{H}$ & 1.170674 & -1.062337 & -2.293374 & $\mathrm{H}$ & -0.656042 & 1.357469 & -2.382234 \\
\hline $\mathrm{C}$ & -0.227976 & 1.494518 & -0.381111 & $\mathrm{H}$ & -1.180390 & 1.866395 & 1.292943 \\
\hline $\mathrm{H}$ & 1.824319 & -1.440217 & 1.395037 & $\mathrm{O}$ & 0.695511 & 0.318366 & 2.159277 \\
\hline $\mathrm{O}$ & -0.646749 & -0.922675 & 2.242303 & $\mathrm{O}$ & 2.134513 & 0.968705 & -2.310342 \\
\hline $\mathrm{O}$ & -1.451525 & -1.751788 & -2.318736 & $\mathrm{C}$ & -2.866854 & 0.112721 & 0.064852 \\
\hline $\mathrm{C}$ & -0.000339 & 2.050328 & 0.903532 & $\mathrm{C}$ & -2.869610 & -0.373510 & 1.379383 \\
\hline $\mathrm{C}$ & -0.886811 & 3.298569 & 1.102479 & $\mathrm{C}$ & -4.001731 & -0.997536 & 1.898710 \\
\hline $\mathrm{C}$ & -1.410215 & 4.006292 & 0.017635 & $\mathrm{C}$ & -5.147197 & -1.136573 & 1.109099 \\
\hline $\mathrm{C}$ & -1.349453 & 3.456543 & -1.262960 & $\mathrm{C}$ & -5.155575 & -0.638776 & -0.194029 \\
\hline $\mathrm{C}$ & -0.763823 & 2.204699 & -1.459283 & $\mathrm{C}$ & -4.019594 & -0.013172 & -0.712658 \\
\hline $\mathrm{H}$ & 0.124552 & 1.495469 & 1.750458 & $\mathrm{H}$ & -1.951983 & -0.275195 & 1.978917 \\
\hline $\mathrm{H}$ & -0.929759 & 3.727105 & 2.111214 & $\mathrm{H}$ & -3.991093 & -1.386457 & 2.924427 \\
\hline $\mathrm{H}$ & -1.866649 & 4.991500 & 0.172485 & $\mathrm{H}$ & -6.036987 & -1.634114 & 1.513813 \\
\hline $\mathrm{H}$ & -1.759941 & 4.007616 & -2.117845 & $\mathrm{H}$ & -6.053809 & -0.741942 & -0.815242 \\
\hline $\mathrm{H}$ & -0.710042 & 1.769275 & -2.466405 & $\mathrm{H}$ & -4.019094 & 0.369099 & -1.742090 \\
\hline $\mathrm{Si}$ & 3.200182 & 1.069888 & 0.175018 & $\mathrm{Si}$ & -0.287885 & -2.019461 & -0.654126 \\
\hline $\mathrm{C}$ & 4.829378 & 0.427651 & -0.532268 & $\mathrm{C}$ & -1.931109 & -2.770215 & -1.197406 \\
\hline $\mathrm{C}$ & 3.325667 & 1.145923 & 2.056193 & $\mathrm{C}$ & 1.062635 & -2.538734 & -1.870761 \\
\hline $\mathrm{C}$ & 2.859273 & 2.780864 & -0.545398 & $\mathrm{C}$ & 0.130537 & -2.692587 & 1.060461 \\
\hline $\mathrm{H}$ & 5.088340 & -0.561807 & -0.112697 & $\mathrm{H}$ & 0.923311 & -2.039837 & -2.848206 \\
\hline $\mathrm{H}$ & 5.660889 & 1.120568 & -0.305829 & $\mathrm{H}$ & 1.032913 & -3.631979 & -2.034917 \\
\hline $\mathrm{H}$ & 4.761537 & 0.323706 & -1.631381 & $\mathrm{H}$ & 2.073182 & -2.285817 & -1.499152 \\
\hline $\mathrm{H}$ & 4.138945 & 1.831438 & 2.358747 & $\mathrm{H}$ & -2.717023 & -2.636636 & -0.431832 \\
\hline $\mathrm{H}$ & 3.553242 & 0.148479 & 2.476174 & $\mathrm{H}$ & -2.302825 & -2.351670 & -2.150402 \\
\hline $\mathrm{H}$ & 2.389701 & 1.506408 & 2.519816 & $\mathrm{H}$ & -1.776706 & -3.855771 & -1.347866 \\
\hline $\mathrm{H}$ & 1.982333 & 3.267717 & -0.082812 & $\mathrm{H}$ & -0.551071 & -2.295485 & 1.834044 \\
\hline $\mathrm{H}$ & 2.689346 & 2.735813 & -1.637534 & $\mathrm{H}$ & 0.022738 & -3.793604 & 1.040745 \\
\hline $\mathrm{H}$ & 3.743106 & 3.424161 & -0.374426 & $\mathrm{H}$ & 1.167841 & -2.465413 & 1.365754 \\
\hline \multicolumn{4}{|c|}{ Cycloadduct 11e-1,5-endo } & \multicolumn{4}{|c|}{ Cycloadduct 11e-1,4-endo } \\
\hline
\end{tabular}




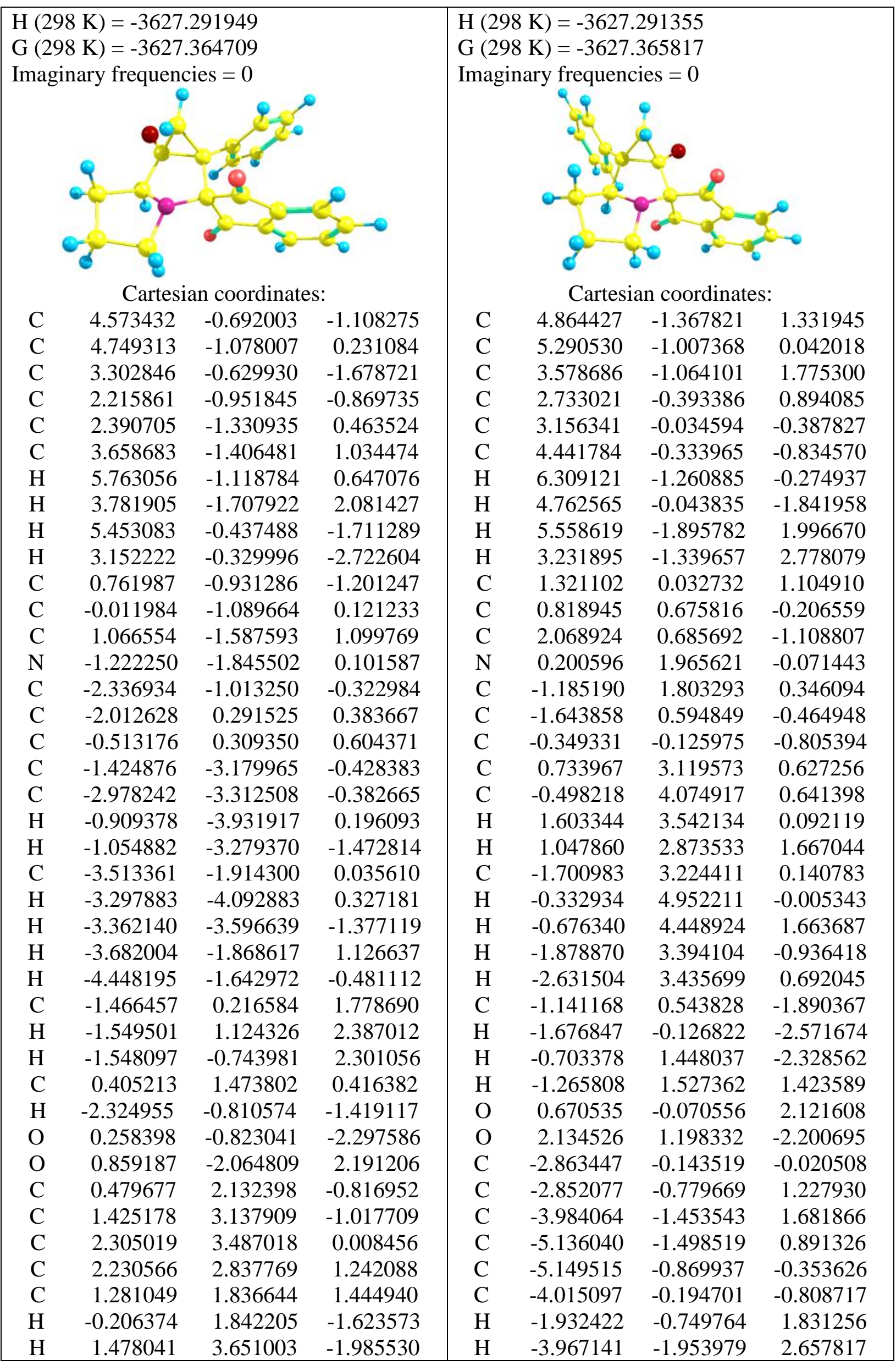




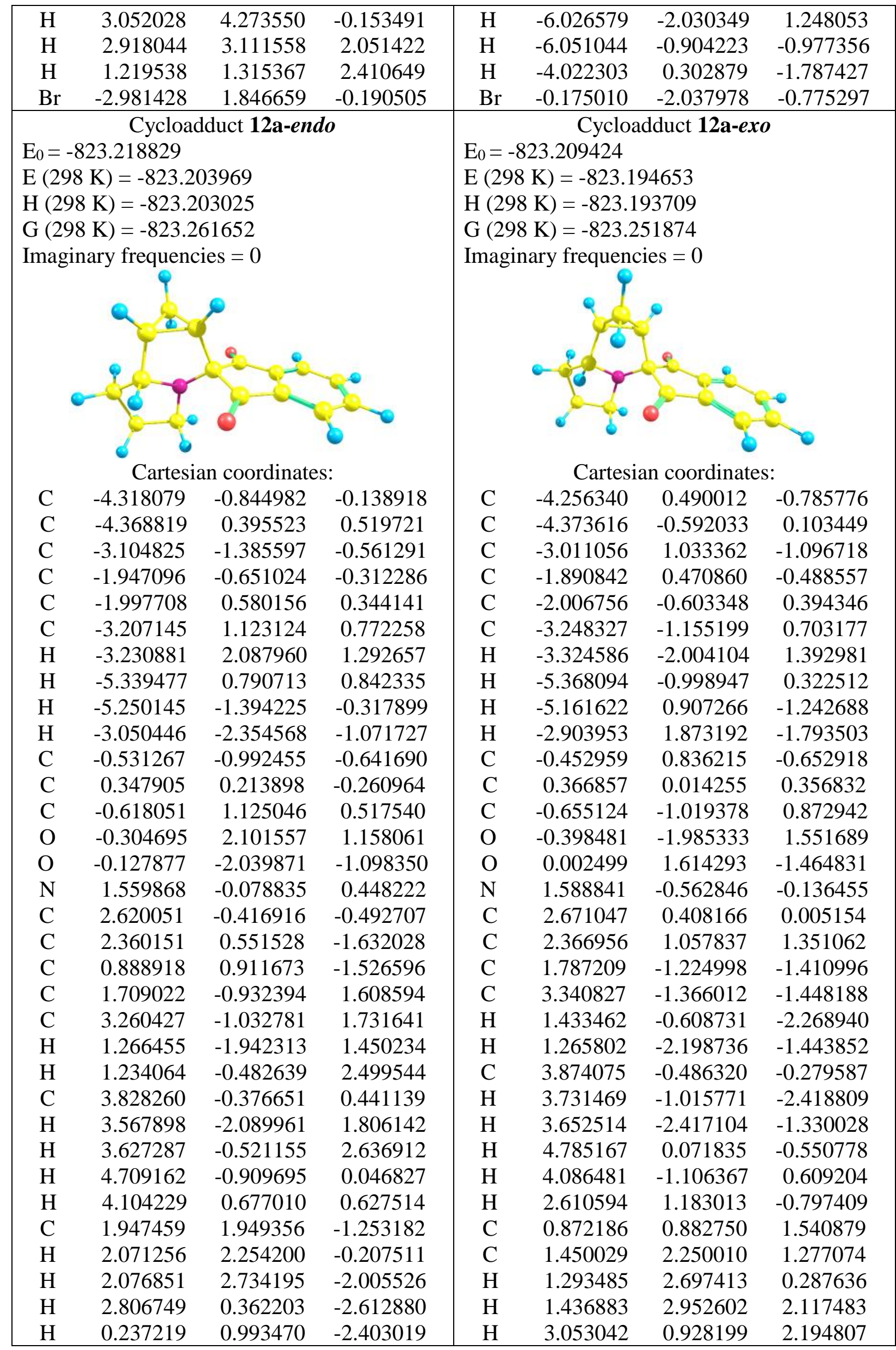




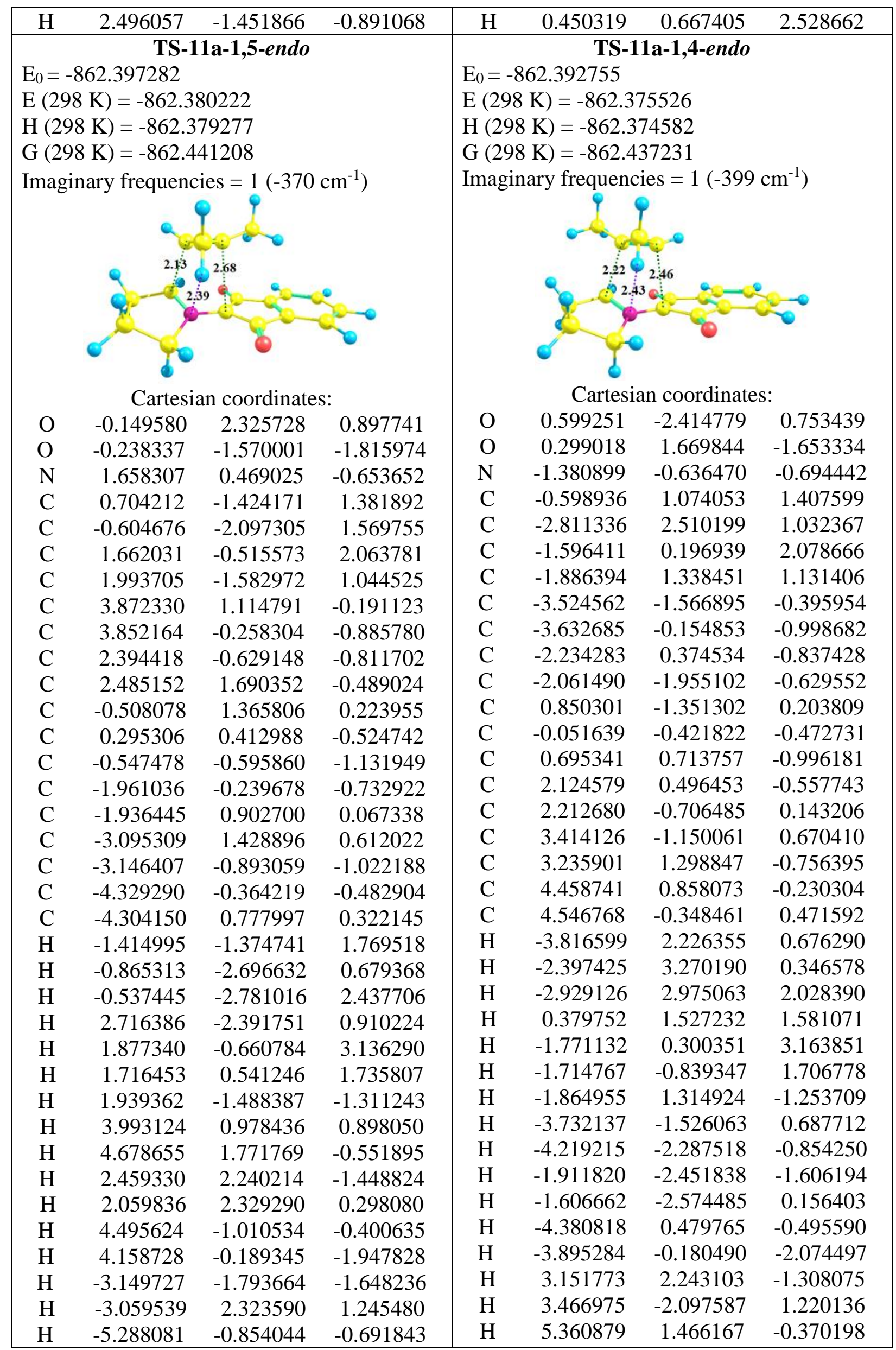




\begin{tabular}{|c|c|c|c|c|c|c|c|}
\hline $\mathrm{H}$ & -5.243252 & 1.167911 & 0.732937 & $\mathrm{H}$ & 5.516499 & -0.669005 & 0.871045 \\
\hline \multicolumn{4}{|c|}{ TS-11a-1,5-exo } & \multicolumn{4}{|c|}{ TS-11a-1,4-exo } \\
\hline \multicolumn{4}{|c|}{$E_{0}=-862.393864$} & \multicolumn{4}{|c|}{$E_{0}=-862.388593$} \\
\hline \multicolumn{4}{|c|}{$E(298 K)=-862.376611$} & \multicolumn{4}{|c|}{$E(298 K)=-862.371249$} \\
\hline \multicolumn{4}{|c|}{$H(298 K)=-862.375667$} & \multicolumn{4}{|c|}{$H(298 K)=-862.370305$} \\
\hline \multicolumn{4}{|c|}{$G(298 K)=-862.438358$} & \multicolumn{4}{|c|}{$\mathrm{G}(298 \mathrm{~K})=-862.433557$} \\
\hline \multicolumn{4}{|c|}{ Imaginary frequencies $=1\left(-367 \mathrm{~cm}^{-1}\right)$} & \multicolumn{4}{|c|}{ Imaginary frequencies $=1\left(-386 \mathrm{~cm}^{-1}\right)$} \\
\hline & & & & & & & \\
\hline \multicolumn{4}{|c|}{ Cartesian coordinates: } & \multicolumn{4}{|c|}{ Cartesian coordinates: } \\
\hline $\mathrm{O}$ & 0.311733 & -2.503781 & 0.670171 & $\mathrm{O}$ & 0.599251 & -2.414779 & 0.753439 \\
\hline $\mathrm{O}$ & 0.102263 & 1.555015 & -1.791377 & $\mathrm{O}$ & 0.299018 & 1.669844 & -1.653334 \\
\hline $\mathrm{N}$ & -1.663095 & -0.580390 & -0.631156 & $\mathrm{~N}$ & -1.380899 & -0.636470 & -0.694442 \\
\hline $\mathrm{C}$ & -0.734956 & 1.290244 & 1.448314 & $\mathrm{C}$ & -0.598936 & 1.074053 & 1.407599 \\
\hline $\mathrm{C}$ & 0.222306 & 0.716893 & 2.429066 & $\mathrm{C}$ & -2.811336 & 2.510199 & 1.032367 \\
\hline $\mathrm{C}$ & -1.116769 & 2.614486 & 0.875930 & $\mathrm{C}$ & -1.596411 & 0.196939 & 2.078666 \\
\hline $\mathrm{C}$ & -2.030073 & 1.427065 & 1.123782 & $\mathrm{C}$ & -1.886394 & 1.338451 & 1.131406 \\
\hline $\mathrm{C}$ & -3.840917 & -1.260456 & -0.018351 & $\mathrm{C}$ & -3.524562 & -1.566895 & -0.395954 \\
\hline $\mathrm{C}$ & -3.885198 & 0.077699 & -0.775326 & $\mathrm{C}$ & -3.632685 & -0.154853 & -0.998682 \\
\hline $\mathrm{C}$ & -2.439847 & 0.493441 & -0.758315 & $\mathrm{C}$ & -2.234283 & 0.374534 & -0.837428 \\
\hline $\mathrm{C}$ & -2.432272 & -1.800916 & -0.293893 & $\mathrm{C}$ & -2.061490 & -1.955102 & -0.629552 \\
\hline $\mathrm{C}$ & 0.579637 & -1.453305 & 0.100554 & $\mathrm{C}$ & 0.850301 & -1.351302 & 0.203809 \\
\hline $\mathrm{C}$ & -0.303514 & -0.480514 & -0.535642 & $\mathrm{C}$ & -0.051639 & -0.421822 & -0.472731 \\
\hline $\mathrm{C}$ & 0.473159 & 0.602377 & -1.104974 & $\mathrm{C}$ & 0.695341 & 0.713757 & -0.996181 \\
\hline $\mathrm{C}$ & 1.906412 & 0.329835 & -0.716243 & $\mathrm{C}$ & 2.124579 & 0.496453 & -0.557743 \\
\hline $\mathrm{C}$ & 1.969392 & -0.880191 & -0.025946 & $\mathrm{C}$ & 2.212680 & -0.706485 & 0.143206 \\
\hline $\mathrm{C}$ & 3.166737 & -1.378183 & 0.458374 & $\mathrm{C}$ & 3.414126 & -1.150061 & 0.670410 \\
\hline $\mathrm{C}$ & 3.042747 & 1.085584 & -0.948855 & $\mathrm{C}$ & 3.235901 & 1.298847 & -0.756395 \\
\hline $\mathrm{C}$ & 4.263271 & 0.591830 & -0.463169 & $\mathrm{C}$ & 4.458741 & 0.858073 & -0.230304 \\
\hline $\mathrm{C}$ & 4.325250 & -0.621732 & 0.228301 & $\mathrm{C}$ & 4.546768 & -0.348461 & 0.471592 \\
\hline $\mathrm{H}$ & 0.181366 & 1.315817 & 3.359691 & $\mathrm{H}$ & -3.816599 & 2.226355 & 0.676290 \\
\hline $\mathrm{H}$ & -0.030980 & -0.329060 & 2.674463 & $\mathrm{H}$ & -2.397425 & 3.270190 & 0.346578 \\
\hline $\mathrm{H}$ & 1.262008 & 0.757301 & 2.057275 & $\mathrm{H}$ & -2.929126 & 2.975063 & 2.028390 \\
\hline $\mathrm{H}$ & -3.003553 & 1.233686 & 1.584869 & $\mathrm{H}$ & 0.379752 & 1.527232 & 1.581071 \\
\hline $\mathrm{H}$ & -0.858689 & 2.851440 & -0.172247 & $\mathrm{H}$ & -1.771132 & 0.300351 & 3.163851 \\
\hline $\mathrm{H}$ & -1.135825 & 3.492112 & 1.547332 & $\mathrm{H}$ & -1.714767 & -0.839347 & 1.706778 \\
\hline $\mathrm{H}$ & -2.029932 & 1.371813 & -1.266973 & $\mathrm{H}$ & -1.864955 & 1.314924 & -1.253709 \\
\hline $\mathrm{H}$ & -3.964382 & -1.081096 & 1.064534 & $\mathrm{H}$ & -3.732137 & -1.526063 & 0.687712 \\
\hline $\mathrm{H}$ & -4.623578 & -1.965651 & -0.337937 & $\mathrm{H}$ & -4.219215 & -2.287518 & -0.854250 \\
\hline $\mathrm{H}$ & -2.395766 & -2.476981 & -1.167763 & $\mathrm{H}$ & -1.911820 & -2.451838 & -1.606194 \\
\hline $\mathrm{H}$ & -1.960301 & -2.311179 & 0.557571 & $\mathrm{H}$ & -1.606662 & -2.574485 & 0.156403 \\
\hline $\mathrm{H}$ & -4.539042 & 0.832144 & -0.307358 & $\mathrm{H}$ & -4.380818 & 0.479765 & -0.495590 \\
\hline $\mathrm{H}$ & -4.218343 & -0.048663 & -1.823758 & $\mathrm{H}$ & -3.895284 & -0.180490 & -2.074497 \\
\hline $\mathrm{H}$ & 2.980310 & 2.035481 & -1.493568 & $\mathrm{H}$ & 3.151773 & 2.243103 & -1.308075 \\
\hline $\mathrm{H}$ & 3.197003 & -2.330437 & 1.001818 & $\mathrm{H}$ & 3.466975 & -2.097587 & 1.220136 \\
\hline $\mathrm{H}$ & 5.184133 & 1.164624 & -0.627533 & $\mathrm{H}$ & 5.360879 & 1.466167 & -0.370198 \\
\hline
\end{tabular}




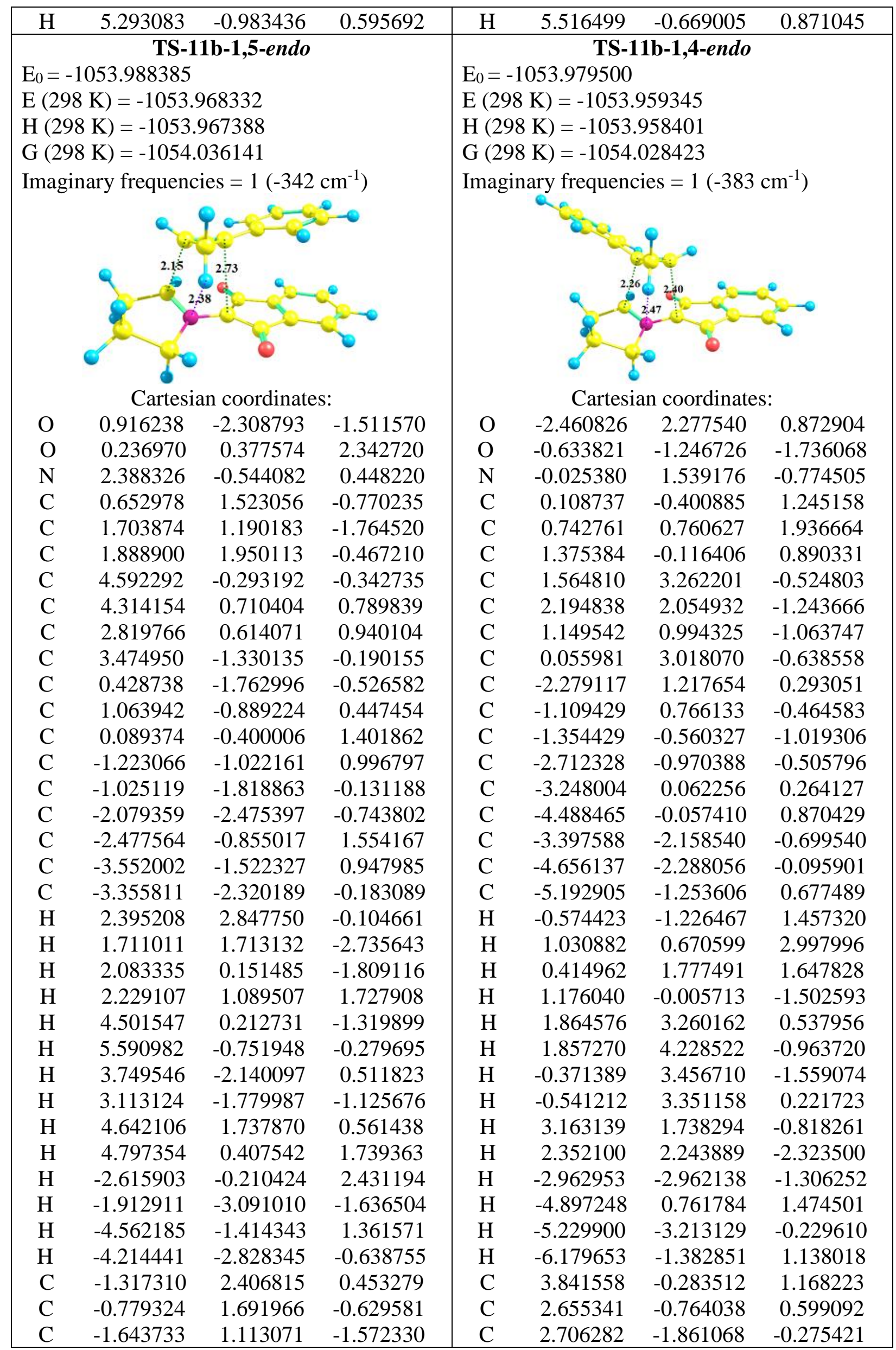




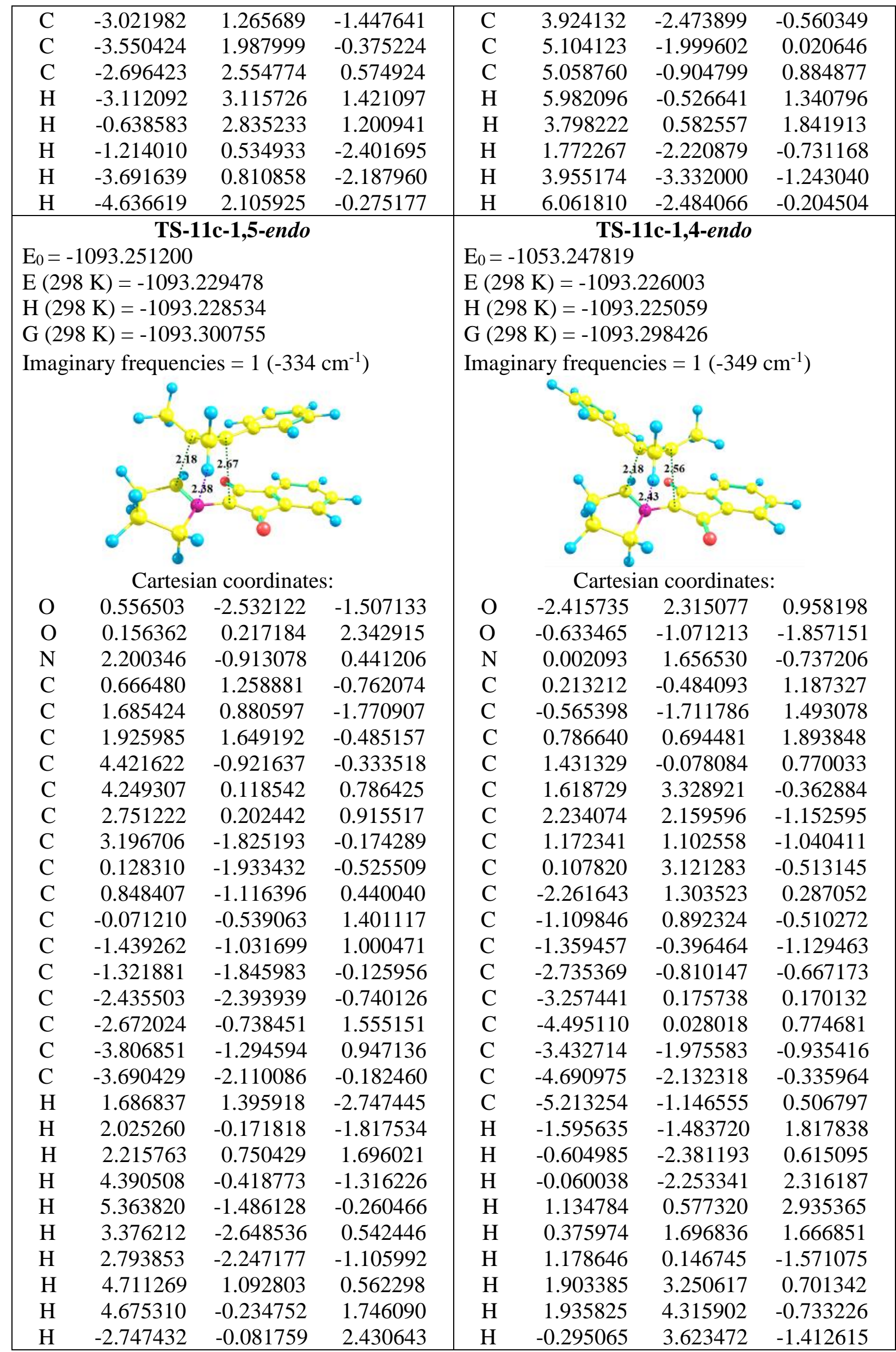




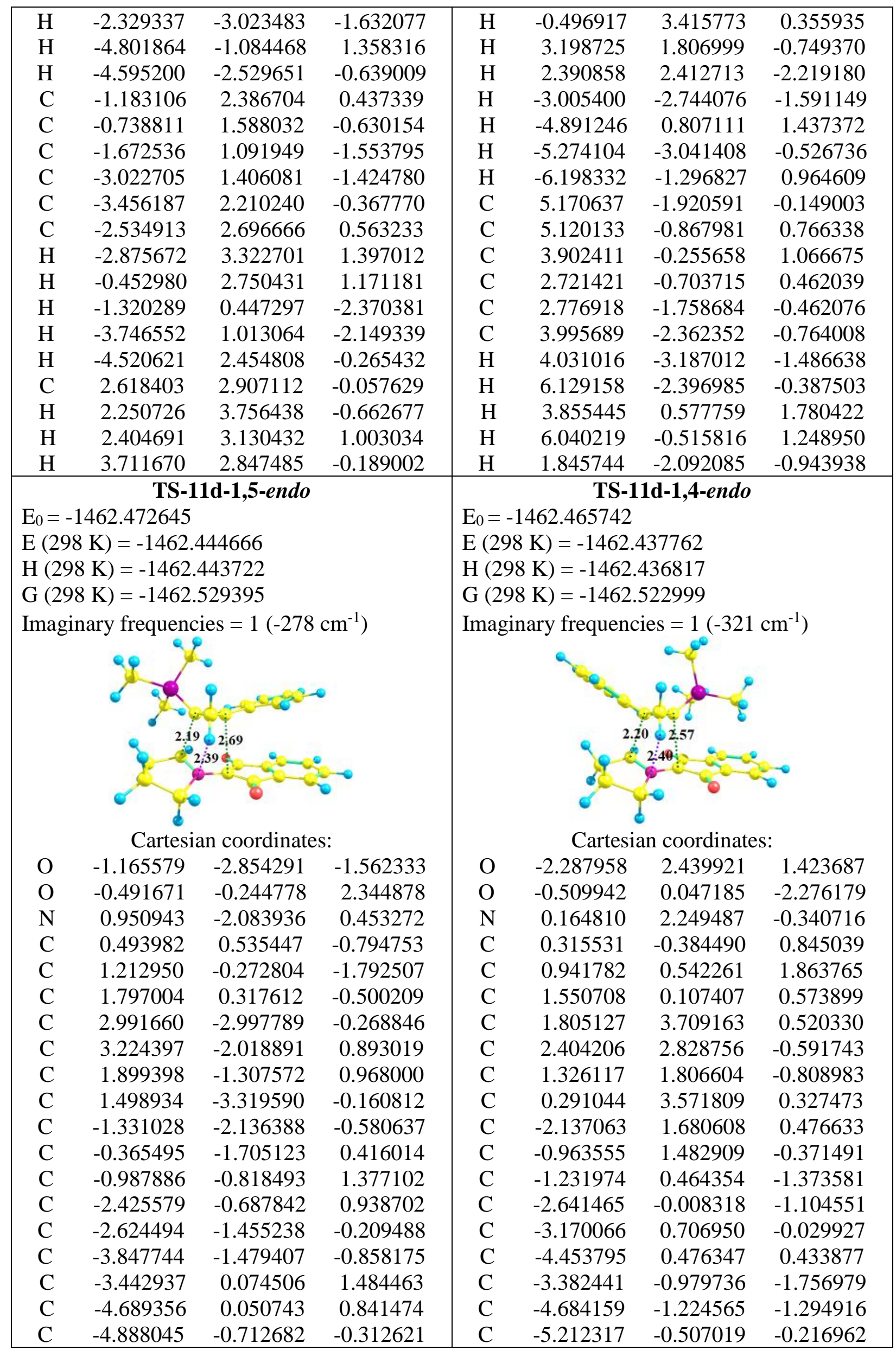




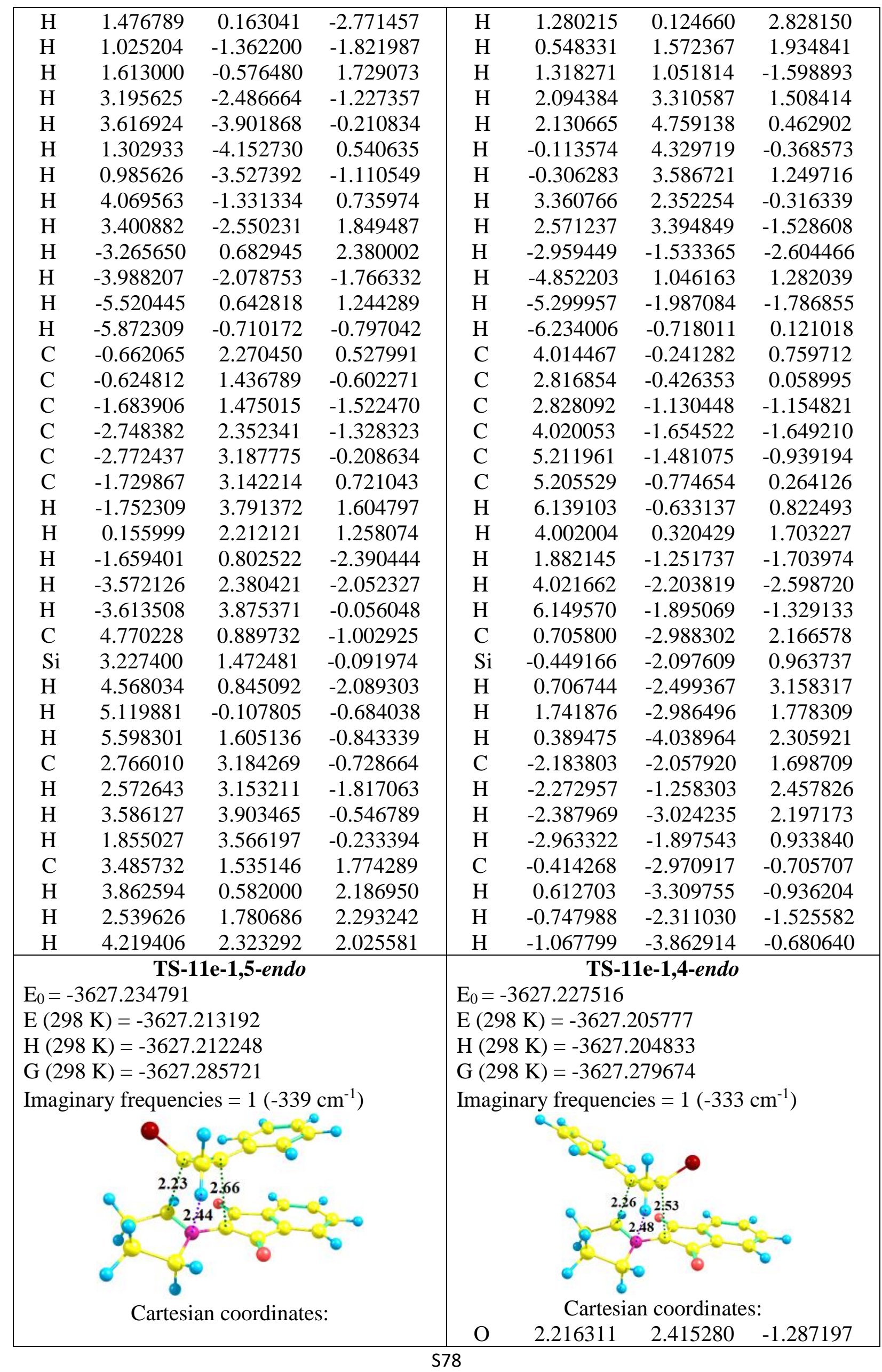




\begin{tabular}{|c|c|c|c|c|c|c|c|}
\hline $\mathrm{O}$ & 0.745563 & 2.857196 & -1.499419 & $\mathrm{O}$ & 0.586063 & -0.331091 & 2.226972 \\
\hline $\mathrm{O}$ & 0.171932 & 0.095701 & 2.318047 & $\mathrm{~N}$ & -0.164766 & 2.043515 & 0.546928 \\
\hline $\mathrm{N}$ & -1.363593 & 1.850528 & 0.415209 & $\mathrm{C}$ & -0.286972 & -0.400986 & -0.934092 \\
\hline $\mathrm{C}$ & -0.557242 & -0.696539 & -0.800065 & $\mathrm{C}$ & -0.883705 & 0.568065 & -1.883900 \\
\hline $\mathrm{C}$ & -1.449754 & -0.048176 & -1.814325 & $\mathrm{C}$ & -1.531974 & 0.000762 & -0.630880 \\
\hline $\mathrm{C}$ & -1.865494 & -0.672816 & -0.510703 & $\mathrm{C}$ & -1.838559 & 3.553752 & -0.140053 \\
\hline $\mathrm{C}$ & -3.457834 & 2.558123 & -0.403833 & $\mathrm{C}$ & -2.400951 & 2.573484 & 0.905021 \\
\hline $\mathrm{C}$ & -3.645248 & 1.528777 & 0.725324 & $\mathrm{C}$ & -1.309494 & 1.548454 & 0.995046 \\
\hline $\mathrm{C}$ & -2.251826 & 0.999232 & 0.911271 & $\mathrm{C}$ & -0.320150 & 3.425407 & 0.016182 \\
\hline $\mathrm{C}$ & -2.006154 & 3.019007 & -0.243885 & $\mathrm{C}$ & 2.109885 & 1.572164 & -0.407423 \\
\hline $\mathrm{C}$ & 0.944079 & 2.155547 & -0.514278 & $\mathrm{C}$ & 0.968591 & 1.287148 & 0.461941 \\
\hline $\mathrm{C}$ & -0.020806 & 1.598925 & 0.425952 & $\mathrm{C}$ & 1.286893 & 0.187887 & 1.365305 \\
\hline $\mathrm{C}$ & 0.650765 & 0.754756 & 1.399738 & $\mathrm{C}$ & 2.685957 & -0.238100 & 1.007033 \\
\hline $\mathrm{C}$ & 2.113332 & 0.806577 & 1.040145 & $\mathrm{C}$ & 3.157401 & 0.555175 & -0.038629 \\
\hline $\mathrm{C}$ & 2.283810 & 1.623710 & -0.077798 & $\mathrm{C}$ & 4.407057 & 0.343143 & -0.598958 \\
\hline $\mathrm{C}$ & 3.530171 & 1.811020 & -0.652351 & $\mathrm{C}$ & 3.446954 & -1.268600 & 1.534123 \\
\hline $\mathrm{C}$ & 3.181857 & 0.151173 & 1.626553 & $\mathrm{C}$ & 4.716436 & -1.484680 & 0.982716 \\
\hline $\mathrm{C}$ & 4.449937 & 0.340609 & 1.059124 & $\mathrm{C}$ & 5.188705 & -0.691765 & -0.069133 \\
\hline $\mathrm{C}$ & 4.621439 & 1.157870 & -0.062807 & $\mathrm{H}$ & -1.234804 & 0.222566 & -2.871079 \\
\hline $\mathrm{H}$ & -1.597269 & -0.571850 & -2.773979 & $\mathrm{H}$ & -0.498010 & 1.603936 & -1.870883 \\
\hline $\mathrm{H}$ & -1.484939 & 1.054740 & -1.884905 & $\mathrm{H}$ & -1.285477 & 0.691151 & 1.672861 \\
\hline $\mathrm{H}$ & -1.927883 & 0.299371 & 1.687019 & $\mathrm{H}$ & -2.139060 & 3.230032 & -1.152578 \\
\hline $\mathrm{H}$ & -3.590509 & 2.066266 & -1.383325 & $\mathrm{H}$ & -2.180222 & 4.589535 & 0.007683 \\
\hline $\mathrm{H}$ & -4.163784 & 3.400411 & -0.342784 & $\mathrm{H}$ & 0.082798 & 4.114769 & 0.780806 \\
\hline $\mathrm{H}$ & -1.910871 & 3.875361 & 0.449357 & $\mathrm{H}$ & 0.264366 & 3.541410 & -0.907037 \\
\hline $\mathrm{H}$ & -1.477446 & 3.259524 & -1.177030 & $\mathrm{H}$ & -3.362222 & 2.114507 & 0.617208 \\
\hline $\mathrm{H}$ & -4.356058 & 0.721788 & 0.480611 & $\mathrm{H}$ & -2.542455 & 3.050767 & 1.894252 \\
\hline $\mathrm{H}$ & -3.986237 & 1.999354 & 1.668113 & $\mathrm{H}$ & 3.056725 & -1.889800 & 2.349302 \\
\hline $\mathrm{H}$ & 3.028046 & -0.499689 & 2.496316 & $\mathrm{H}$ & 4.762611 & 0.969250 & -1.426116 \\
\hline $\mathrm{H}$ & 3.648385 & 2.448141 & -1.537577 & $\mathrm{H}$ & 5.349753 & -2.289266 & 1.375401 \\
\hline $\mathrm{H}$ & 5.321246 & -0.161994 & 1.496508 & $\mathrm{H}$ & 6.185029 & -0.886475 & -0.483830 \\
\hline $\mathrm{H}$ & 5.624809 & 1.285980 & -0.486645 & $\mathrm{C}$ & -4.004624 & -0.229757 & -0.845400 \\
\hline $\mathrm{C}$ & 0.846117 & -2.369336 & 0.349808 & $\mathrm{C}$ & -2.805068 & -0.584408 & -0.216167 \\
\hline $\mathrm{C}$ & 0.677070 & -1.444034 & -0.693230 & $\mathrm{C}$ & -2.819292 & -1.511706 & 0.837117 \\
\hline $\mathrm{C}$ & 1.716845 & -1.230429 & -1.611082 & $\mathrm{C}$ & -4.021438 & -2.084131 & 1.244823 \\
\hline $\mathrm{C}$ & 2.904350 & -1.948453 & -1.498895 & $\mathrm{C}$ & -5.216212 & -1.738655 & 0.606860 \\
\hline $\mathrm{C}$ & 3.065751 & -2.874787 & -0.465577 & $\mathrm{C}$ & -5.204630 & -0.812929 & -0.437622 \\
\hline $\mathrm{C}$ & 2.037382 & -3.082425 & 0.457926 & $\mathrm{H}$ & -6.140902 & -0.537995 & -0.938385 \\
\hline $\mathrm{H}$ & 2.167488 & -3.806785 & 1.271050 & $\mathrm{H}$ & -3.986776 & 0.503273 & -1.662842 \\
\hline $\mathrm{H}$ & 0.033365 & -2.512297 & 1.073152 & $\mathrm{H}$ & -1.871889 & -1.769634 & 1.332445 \\
\hline $\mathrm{H}$ & 1.581821 & -0.486450 & -2.407603 & $\mathrm{H}$ & -4.028049 & -2.808607 & 2.068188 \\
\hline $\mathrm{H}$ & 3.715459 & -1.778931 & -2.217442 & $\mathrm{H}$ & -6.161439 & -2.191764 & 0.928843 \\
\hline $\mathrm{H}$ & 4.003329 & -3.437128 & -0.376197 & $\mathrm{Br}$ & 0.643301 & -2.018688 & -1.016697 \\
\hline $\mathrm{Br}$ & -3.115287 & -1.958933 & 0.075080 & \multicolumn{4}{|c|}{ TS-12a-exo } \\
\hline $\begin{array}{l}\mathrm{E}_{0}= \\
\mathrm{E}(29 \\
\mathrm{H}(29 \\
\mathrm{G}(29 \\
\text { Imag }\end{array}$ & $\begin{array}{l}\text { TS } \\
23.129148 \\
\mathrm{~K})=-823.1 \\
\mathrm{~K})=-823.1 \\
\mathrm{~K})=-823.1 \\
\text { lary frequenc }\end{array}$ & $\begin{array}{l}\text { 12a-endo } \\
3593 \\
12649 \\
71489 \\
\text { les }=1(-400\end{array}$ & $\left(m^{-1}\right)$ & $\begin{array}{l}\mathrm{E}_{0}= \\
\mathrm{E}(29 \\
\mathrm{H}(29 \\
\mathrm{G}(29 \\
\text { Imag }\end{array}$ & $\begin{array}{l}\mathbf{T} \\
23.126044 \\
\mathrm{~K})=-823.1 \\
\mathrm{~K})=-823.1 \\
\mathrm{~K})=-823.1 \\
\text { ary frequen }\end{array}$ & $\begin{array}{l}\text {-12a-exo } \\
0390 \\
9446 \\
68663 \\
\text { es }=1(-392\end{array}$ & $\left.\mathrm{cm}^{-1}\right)$ \\
\hline
\end{tabular}




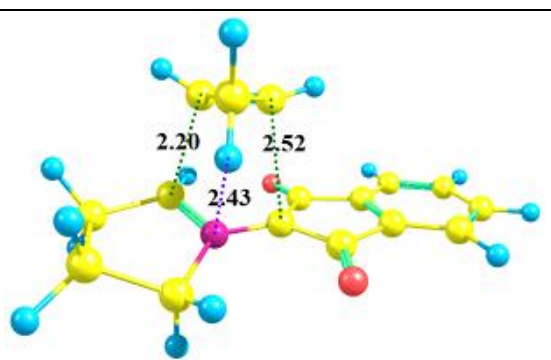

Cartesian coordinates:

$\mathrm{O}$

$\mathrm{O}$

$-0.223863$

2.416251

0.001666

.256836

$\mathrm{N} \quad 1.611037$

$-2.246981$

0.102602

$-0.902223$

$-0.665409$

0.626584

$-0.766248$

1.874146

C $\quad 1.628596$

C $\quad 1.908676$

C $\quad 3.821126$

C 3.818352

C 2.369349

C $\quad 2.416108$

C $\quad-0.563756$

C $\quad 0.258809$

C $\quad-0.577702$

C -1.991534

C -1.981017

C -3.147431

C -3.170713

C -4.359311

C $\quad-4.347967$

$\mathrm{H} \quad-0.382287$

H 1.790845

H 1.773523

H 1.922106

H 3.967929

H 4.604628

$\mathrm{H} \quad 2.359351$

$\mathrm{H} \quad 1.990030$

H 4.474486

$\mathrm{H} \quad 4.122691$

$\mathrm{H} \quad-3.165065$

$\mathrm{H} \quad-3.122613$

H $\quad-5.312650$

H $\quad-5.292448$

H 2.588779
0.274780

$-1.119703$

0.906547

$-0.628621$

$-0.960956$

1.307801

1.255625

0.084682

$-1.095867$

$-0.627197$

0.743498

1.436305

$-1.353347$

$-0.661557$

0.711969

$-1.112029$

0.541242

1.120887

$-1.952958$

1.202942

1.384436

1.451564

2.191052

$-1.126016$

$-0.972468$

$-2.430530$

2.513618

$-1.203595$

1.227096

$-1.953883$
2.234552

$-0.535542$

$-0.647359$

$-0.419874$

$-0.994097$

$-0.185757$

$-0.473392$

$-0.626310$

$-0.370243$

$-0.110125$

0.168427

$-0.363620$

$-0.087390$

0.174528

2.106780

3.292136

1.534867

$-0.514322$

0.518016

$-1.143568$

$-2.089299$

$-0.498036$

0.086125

$-1.655409$

$-0.570136$

$-0.076052$

0.387082

1.910767
1.728821

0.372775

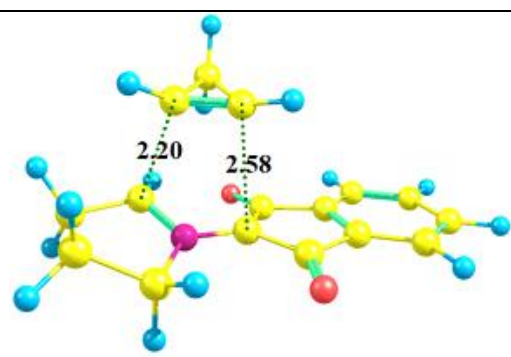

Cartesian coordinates:

\begin{tabular}{rrrr}
$\mathrm{O}$ & -0.316267 & 2.525423 & 0.586126 \\
$\mathrm{O}$ & -0.132497 & -1.746643 & -1.478309 \\
$\mathrm{~N}$ & 1.645410 & 0.475820 & -0.536202 \\
$\mathrm{C}$ & 0.698799 & -1.103221 & 1.677171 \\
$\mathrm{C}$ & 1.054691 & -2.510243 & 1.315734 \\
$\mathrm{C}$ & 1.996579 & -1.330025 & 1.446551 \\
$\mathrm{C}$ & 3.834560 & 1.224383 & -0.047848 \\
$\mathrm{C}$ & 3.869640 & -0.185459 & -0.661816 \\
$\mathrm{C}$ & 2.427272 & -0.598094 & -0.581172 \\
$\mathrm{C}$ & 2.414766 & 1.728022 & -0.338367 \\
$\mathrm{C}$ & -0.588606 & 1.417262 & 0.146917 \\
$\mathrm{C}$ & 0.292720 & 0.381088 & -0.395191 \\
$\mathrm{C}$ & -0.496848 & -0.743116 & -0.870229 \\
$\mathrm{C}$ & -1.925950 & -0.428133 & -0.501717 \\
$\mathrm{C}$ & -1.978639 & 0.833201 & 0.089448 \\
$\mathrm{C}$ & -3.174774 & 1.380512 & 0.523128 \\
$\mathrm{C}$ & -3.069640 & -1.186758 & -0.686699 \\
$\mathrm{C}$ & -4.287127 & -0.643304 & -0.251882 \\
$\mathrm{C}$ & -4.339266 & 0.620227 & 0.344721 \\
$\mathrm{H}$ & -0.013594 & -0.532094 & 2.275833 \\
$\mathrm{H}$ & 0.822459 & -2.878761 & 0.300219 \\
$\mathrm{H}$ & 1.019953 & -3.290799 & 2.096229 \\
$\mathrm{H}$ & 2.009892 & -1.534158 & -0.963772 \\
$\mathrm{H}$ & 3.989260 & 1.160726 & 1.043799 \\
$\mathrm{H}$ & 4.602052 & 1.896363 & -0.461455 \\
$\mathrm{H}$ & 2.349398 & 2.314791 & -1.272563 \\
$\mathrm{H}$ & 1.959709 & 2.315304 & 0.471750 \\
$\mathrm{H}$ & 4.529736 & -0.886477 & -0.124310 \\
$\mathrm{H}$ & 4.191509 & -0.171132 & -1.721247 \\
$\mathrm{H}$ & -3.014028 & -2.175551 & -1.157996 \\
$\mathrm{H}$ & -3.199894 & 2.375051 & 0.985163 \\
$\mathrm{H}$ & -5.213835 & -1.215648 & -0.380809 \\
$\mathrm{H}$ & -5.305572 & 1.020468 & 0.674397 \\
$\mathrm{H}$ & 2.976123 & -1.097121 & 1.873135 \\
& & & \\
\hline
\end{tabular}

Table S6. Relative enthalpy, entropy and free energy change between the reactants $\mathbf{2 a - e}, \mathbf{3 a}$ and their corresponding transition states (enthalpies and free energies of activation $\Delta H_{\#}$ and $\Delta G_{\# \text {, }}$ respectively, are given in $\mathrm{kcal} / \mathrm{mol}$, entropies of activation $\Delta S_{\#}$ are presented in $\mathrm{cal} / \mathrm{mol} \cdot \mathrm{K}$ ).

\begin{tabular}{|c|c|c|c|c|}
\hline Reaction $^{a}$ & Transition & $\Delta \boldsymbol{H}_{\#,}, \mathbf{k c a l} / \mathbf{m o l}$ & $\Delta \boldsymbol{S}_{\#,}, \mathbf{c a l} / \mathbf{m o l} \cdot \mathbf{K}$ & $\Delta \boldsymbol{G}_{\#}, \mathbf{k c a l} / \mathbf{m o l}$ \\
\hline $\mathbf{3 a}+$ DHPO $(\mathbf{6})$ & $\mathbf{3 a}+\mathbf{6} \rightarrow$ TS-endo & 2.4 & -46.7 & 16.3 \\
\hline $\mathbf{3 a}+$ DHPO $(\mathbf{6})$ & $\mathbf{3 a}+\mathbf{6} \rightarrow$ TS-exo & 4.4 & -45.9 & 18.1 \\
\hline
\end{tabular}




\begin{tabular}{|c|c|c|c|c|}
\hline $\mathbf{2 a}+\operatorname{DHPO}(\mathbf{6})$ & $2 \mathbf{a}+\mathbf{6} \rightarrow$ TS-1,5-endo & 2.2 & -50.6 & 17.3 \\
\hline $\mathbf{2 a}+$ DHPO (6) & $\mathbf{2 a}+\mathbf{6} \rightarrow$ TS-1,4-endo & 5.1 & -49.1 & 19.8 \\
\hline $\mathbf{2 a}+$ DHPO (6) & $\mathbf{2 a}+\mathbf{6} \rightarrow \mathrm{TS}-1,5-$ exo & 4.4 & -49.0 & 19.1 \\
\hline $\mathbf{2 a}+\operatorname{DHPO}(\mathbf{6})$ & $\mathbf{2 a}+\mathbf{6} \rightarrow \mathrm{TS}-1,4-$ exo & 7.8 & -47.8 & 22.1 \\
\hline $\mathbf{2 b}+\mathrm{DHPO}(\mathbf{6})$ & $\mathbf{2 b}+\mathbf{6} \rightarrow \mathrm{TS}-1,5$-endo & -3.7 & -54.1 & 12.4 \\
\hline $\mathbf{2 b}+$ DHPO (6) & $\mathbf{2 b}+\mathbf{6} \rightarrow \mathrm{TS}-1,4-$ endo & 1.9 & -51.4 & 17.3 \\
\hline $\mathbf{2 c}+$ DHPO (6) & $\mathbf{2 c}+\mathbf{6} \rightarrow \mathrm{TS}-1,5$-endo & -0.6 & -56.4 & 16.2 \\
\hline $2 c+$ DHPO (6) & $\mathbf{2 c}+\mathbf{6} \rightarrow \mathrm{TS}-1,4-$ endo & 1.6 & -54.0 & 17.6 \\
\hline $\mathbf{2 d}+$ DHPO (6) & $\mathbf{2 d}+\mathbf{6} \rightarrow \mathrm{TS}-1,5$-endo & -4.4 & -60.1 & 13.5 \\
\hline $\mathbf{2 d}+$ DHPO (6) & $\mathbf{2 d}+\mathbf{6} \rightarrow \mathrm{TS}-1,4$-endo & -0.1 & -59.0 & 17.5 \\
\hline $\mathbf{2 e}+\mathrm{DHPO}(\mathbf{6})$ & $\mathbf{2 e}+\mathbf{6} \rightarrow \mathrm{TS}-1,5$-endo & -3.5 & -54.6 & 12.8 \\
\hline $\mathbf{2 e}+$ DHPO (6) & $\mathbf{2 e}+\mathbf{6} \rightarrow \mathrm{TS}-1,4$-endo & 1.1 & -51.7 & 16.6 \\
\hline $\begin{array}{l}\text { a Parent cyclopr } \\
\text { phenylcyclopropen }\end{array}$ & - 3a, 1-methylcyclo & & & 1-me \\
\hline
\end{tabular}

Table S7. Relative enthalpy, entropy and free energy change between the reactants $\mathbf{2 a - e}, \mathbf{3 a}$ and their corresponding products $\mathbf{1 1 a}-\mathbf{e}, \mathbf{1 2 a}(\Delta H$ and $\Delta G$ are given in $\mathrm{kcal} / \mathrm{mol}, \Delta S$ are presented in $\mathrm{cal} / \mathrm{mol} \cdot \mathrm{K})$.

\begin{tabular}{|c|c|c|c|c|}
\hline Reaction $^{a}$ & Transition & $\Delta H, \mathrm{kcal} / \mathrm{mol}$ & $\Delta S, \mathrm{cal} / \mathrm{mol} \cdot \mathrm{K}$ & $\Delta G, \mathrm{kcal} / \mathrm{mol}$ \\
\hline $\mathbf{3 a}+\mathrm{DHPO}(\mathbf{6})$ & $3 \mathrm{a}+6 \rightarrow 12 \mathrm{a}-$ endo & -54.3 & -47.1 & -40.3 \\
\hline $\mathbf{3 a}+\mathrm{DHPO}(\mathbf{6})$ & $3 a+6 \rightarrow 12 a-e x o$ & -48.5 & -48.1 & -34.1 \\
\hline $\mathbf{2 a}+\mathrm{DHPO}(\mathbf{6})$ & $2 \mathrm{a}+6 \rightarrow 11 \mathrm{a}-1,5-e n d o$ & -52.1 & -52.4 & -36.5 \\
\hline $\mathbf{2 a}+\mathrm{DHPO}(\mathbf{6})$ & $2 \mathrm{a}+6 \rightarrow 11 \mathrm{a}-1,4-e n d o$ & -51.3 & -51.1 & -36.1 \\
\hline $\mathbf{2 a}+\mathrm{DHPO}(\mathbf{6})$ & $2 a+6 \rightarrow 11 a-1,5-e x o$ & -45.7 & -53.3 & -29.8 \\
\hline $\mathbf{2 a}+\mathrm{DHPO}(\mathbf{6})$ & $2 a+6 \rightarrow$ 11a-1,4-exo & -45.5 & -51.8 & -30.1 \\
\hline $\mathbf{2 b}+\mathrm{DHPO}(\mathbf{6})$ & $2 \mathrm{~b}+6 \rightarrow 11 \mathrm{~b}-1,5$-endo & -51.9 & -52.2 & -36.4 \\
\hline $\mathbf{2 b}+\mathrm{DHPO}(\mathbf{6})$ & $2 \mathrm{~b}+6 \rightarrow 11 \mathrm{~b}-1,4-e n d o$ & -51.0 & -49.4 & -36.3 \\
\hline $2 c+$ DHPO (6) & $2 c+6 \rightarrow 11 c-1,5-e n d o$ & -49.8 & -58.1 & -32.5 \\
\hline $2 c$ + DHPO (6) & $2 c+6 \rightarrow 11 c-1,4-e n d o$ & -49.9 & -55.6 & -33.3 \\
\hline $\mathbf{2 d}+$ DHPO (6) & $2 \mathrm{~d}+6 \rightarrow 11 \mathrm{~d}-1,5-e n d o$ & -47.4 & -57.3 & -30.3 \\
\hline $\mathbf{2 d}+$ DHPO (6) & $2 \mathrm{~d}+6 \rightarrow$ 11d-1,4-endo & -49.0 & -63.2 & -30.2 \\
\hline $\mathbf{2 e}+\mathrm{DHPO}(\mathbf{6})$ & $2 \mathrm{e}+6 \rightarrow 11 \mathrm{e}-1,5-e n d o$ & -53.5 & -56.1 & -36.8 \\
\hline $2 e+$ DHPO (6) & $2 \mathrm{e}+6 \rightarrow 11 \mathrm{e}-1,4-e n d o$ & -53.2 & -52.5 & -37.5 \\
\hline
\end{tabular}




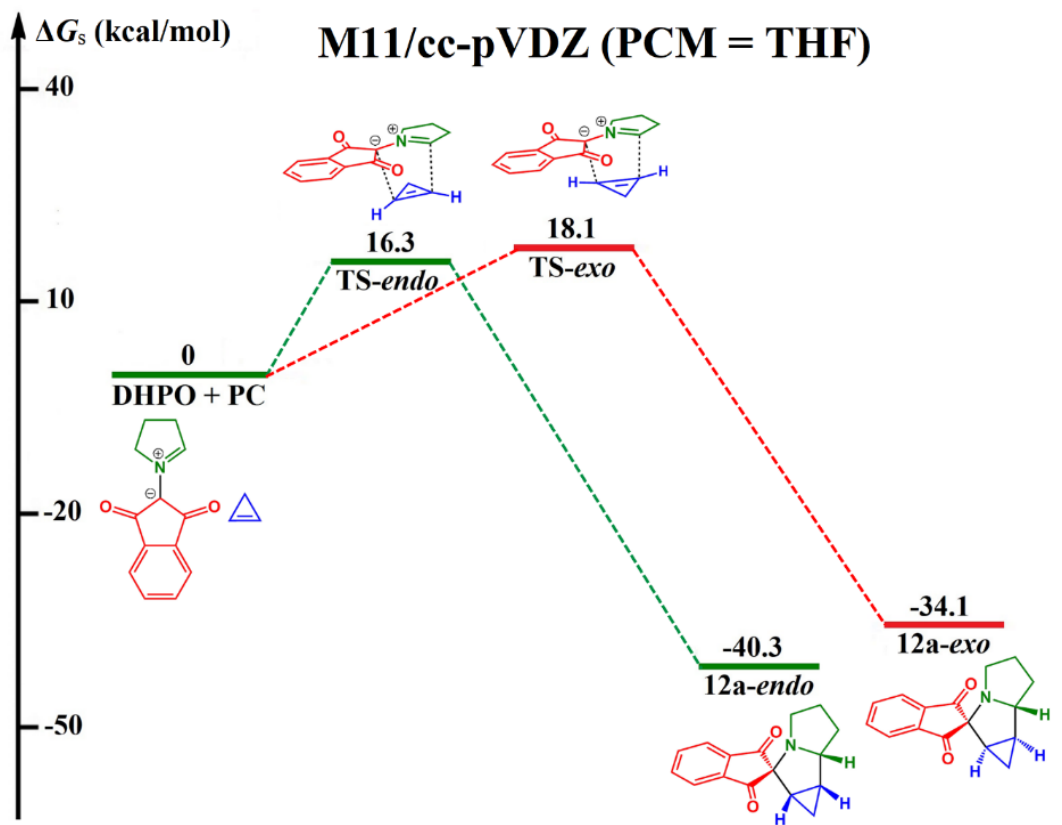

Figure S92. Free energy profile for the 1,3-dipolar cycloaddition reaction of parent cyclopropene (3a) with DHPO (6).

\section{References}

(1) Sheldrick, G. M. SHELXT - Integrated space-group and crystal-structure determination. Acta Cryst. 2015, A71, 3-8.

(2) (a) Palatinus, L.; Chapuis, G. SUPERFLIP - a computer program for the solution of crystal structures by charge flipping in arbitrary dimensions. J. Appl. Cryst. 2007, 40, 786-790. (b) Palatinus, L.; van der Lee, A. Symmetry determination following structure solution in P1. J. Appl. Cryst. 2008, 41, 975-984. (c) Palatinus, L.; Prathapa, S. J.; van Smaalen, S. EDMA: a computer program for topological analysis of discrete electron densities. J. Appl. Cryst. 2012, 45, 575-580.

(3) Sheldrick, G. M. Crystal structure refinement with SHELXL. Acta Cryst. 2015, C71, 3-8.

(4) Dolomanov, O. V.; Bourhis, L. J.; Gildea, R. J.; Howard, J. A. K.; Puschmann, H. J. Appl. Cryst. 2009, 42, 339-341.

(5) CrysAlisPro, Rigaku Oxford Diffraction, Version 1.171.39.46e, 2018.

(6) CrysAlisPro, Agilent Technologies, Version 1.171.36.32, 2013. 